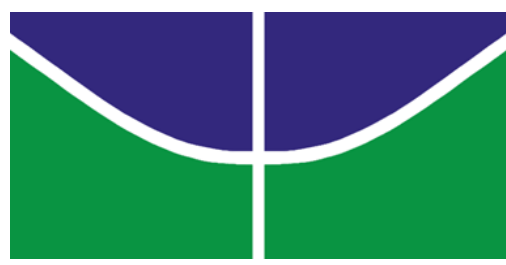

UNIVERSIDADE DE BRASÍLIA

INSTITUTO DE CIÊNCIAS BIOLÓGICAS

PROGRAMA DE PÓS GRADUAÇÃO EM ECOLOGIA

\title{
A CONVENÇÃO SOBRE DIVERSIDADE BIOLÓGICA E O PLANEJAMENTO SISTEMÁTICO PARA A CONSERVAÇÃO NO BRASIL
}

Tese apresentada ao Programa de

Pós Graduação em Ecologia da Universidade de Brasília como prérequisito para a obtenção do título de Doutor em Ecologia.

\section{GIOVANA BOTTURA}

Orientador: Dr. Ricardo Bomfim Machado 
Ficha catalográfica elaborada automaticamente, com os dados fornecidos pelo(a) autor(a)

A Convenção sobre Diversidade Biológica e o

Planejamento Sistemático para a Conservação no Brasil

/ Giovana Bottura; orientador Ricardo Bomfim

Machado. -- Brasília, 2016.

$162 \mathrm{p}$.

Tese (Doutorado - Doutorado em Ecologia) -Universidade de Brasília, 2016.

1. Cenários. 2. Espécies ameaçadas. 3. Espécies endêmicas. 4. Mudanças no uso e cobertura da terra. 5. Cerrado. I. Machado, Ricardo Bomfim, orient. II. Título. 
Dedico este trabalho ao Gustavo, a Gabriela e aos meus pais. 
"Há um tempo em que é preciso abandonar as roupas usadas, que já têm a forma do nosso corpo, e esquecer os nossos caminhos que nos levam sempre aos mesmos lugares. É o tempo da travessia e, se não ousarmos fazê-la, teremos ficado, pra sempre, à margem de nós mesmos"

Fernando Pessoa 


\section{AGRADECIMENTOS}

Ao orientador Ricardo B. Machado (Pacheco) pela calma e orientação;

Ao IBAMA e à UnB pelo apoio financeiro, institucional e técnico-científico;

À Ludmilla M. de S. Aguiar pelas primeiras orientações;

Aos colegas do laboratório: Renata Françoso, Bárbara Zimbres, Renata Alquezar, Danilo

Oliveira, Mariana Stein,Thalita Grande e Prof ${ }^{\circ}$. Roberto Cavalcanti pela companhia e

momentos de descontração. Um agradecimento especial ao Yuri Salmona, à Vivian Ribeiro e à

Romina Cardoso, que facilitaram meus caminhos pelos modelos computacionais compartilhando seus aprendizados.

Aos meus amigos de sempre: Kelly Resende, Verusca Cavalcante , Jailton Dias, Raquel Sabaini;

Zuca; Mayra Amboni; Guilherme Déstro, Lila, Valdir Mano e Fabiana Cristina Julião pelo incentivo, suporte emocional e momentos besteirol;

À minha família de sangue: mamãe (Tereza), papai (Olavo), irmãs (Bianca e Daniela), que mesmo distantes sempre serão meu ponto de referência no mundo;

À minha família afetiva: Norma, Ricardo, Mariana, Marquinhos e Giselle que me acolheram e conviveram com minhas preocupações acadêmicas;

Ao Gustavo Pinho, Jailton Dias, Raquel Sabaini, Rodrigo Koblitz, Danilo Oliveira, Ricardo Ayres \& Auristela, por terem me ouvido, lido meus escritos e acalmado meus ânimos;

Ao Gilberto Werneck pela paciência e compreensão;

À Gabriela, que tumultuou minha vida acadêmica mesmo antes de existir e que, ao nascer, me trouxe ao doce, lindo e caótico mundo da maternidade;

Ao Gustavo (marido) pela infinita paciência, ternura, amor e dedicação sem os quais não teria conseguido vencer todas as etapas;

Aos membros da banca pelas críticas construtivas: Prof. Dr. Ludgero C.G. Vieira;

Prof. Dr. Guarino Colli; Prof. Dr. Mário Barroso R. Neto; Prof. Dr. Carlos Eduardo Grelle e Prof. Dr. Ricardo B. Machado;

Agradeço a todos que contribuíram para minha formação: professores, colegas e palestrantes.

Depois de 12 anos afastada da prática acadêmica, voltar foi desafiador. Muitas das metodologias por mim abordadas permeavam meu cotidiano no IBAMA, mas de um jeito superficial. A academia me proporcionou um novo olhar sobre o tema e despertou em mim a estudante há muito adormecida. 


\section{SUMÁRIO}

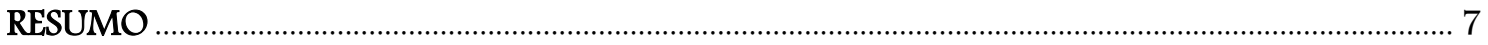

INTRODUÇÃO

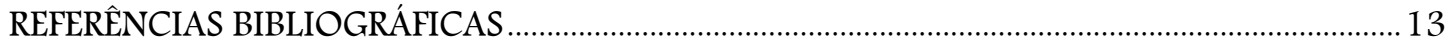

CAPÍTULO 1: THE SCIENCE AND PRACTICE OF CONSERVATION PLANNING IN BRAZIL ........17

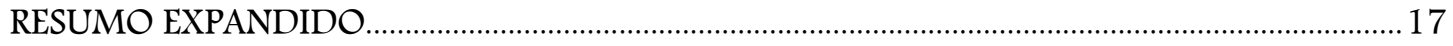

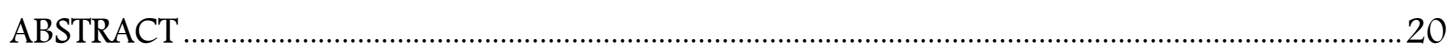

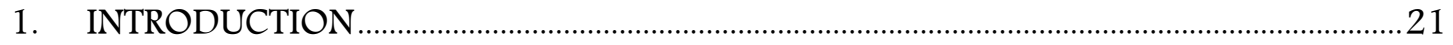

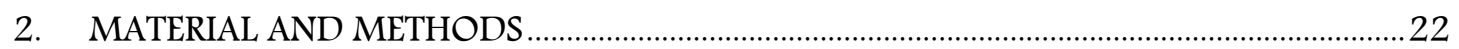

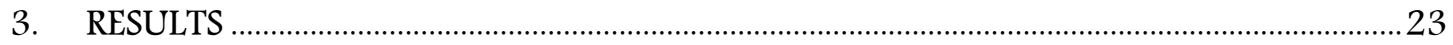

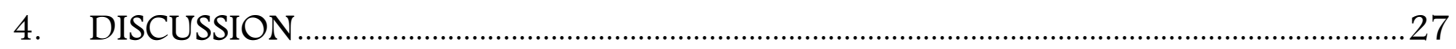

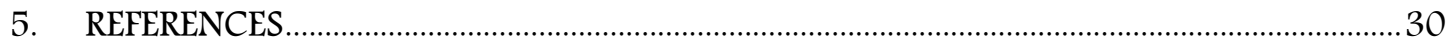

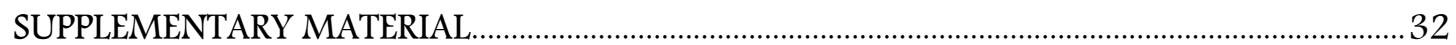

CAPÍTULO 2: CENÁRIOS PARA A CONSERVAÇÃO DA BIODIVERDADE NO BRASIL: AS METAS

PARA A CONVENÇÃO SOBRE DIVERSIDADE BIOLÓGICA SÃO SUFICIENTES? ...............................51

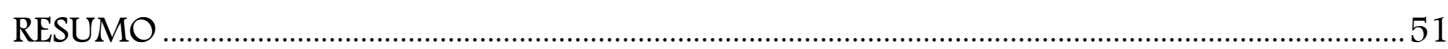

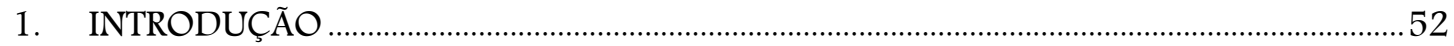

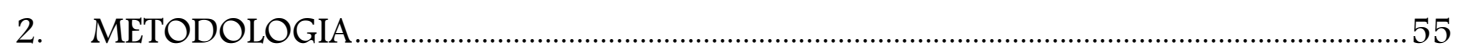

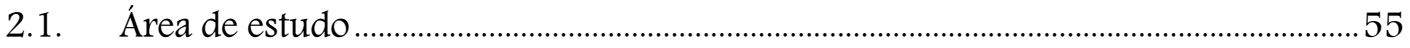

2.2. Construção do modelo de transição potencial .....................................................................56

2.3. Desenho conceitual e elaboração dos cenários ...................................................................57

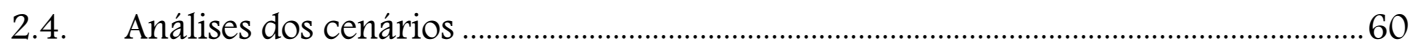

3. RESULTADOS

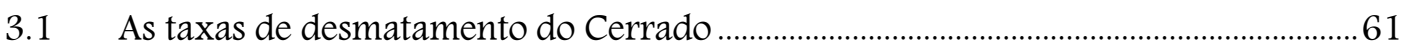

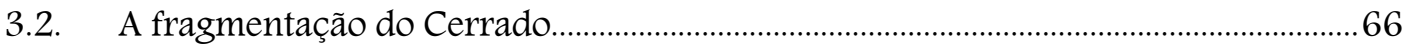

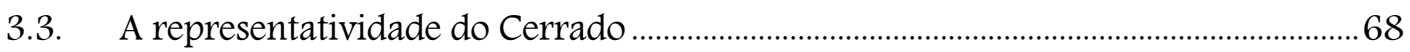

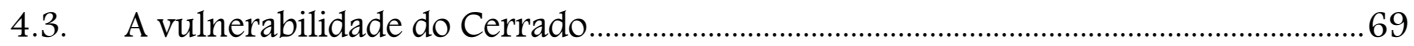

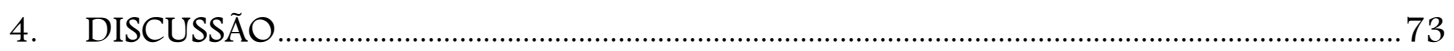


4.3. Os padrões de desmatamento, suas forçantes e os modelos de LUCC ...........................73

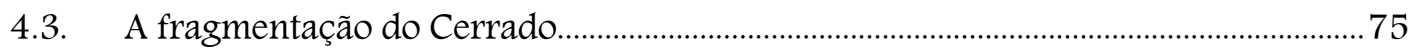

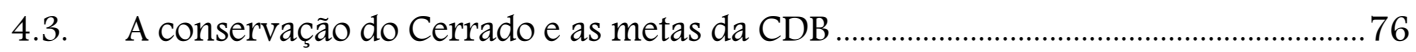

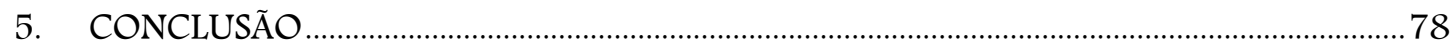

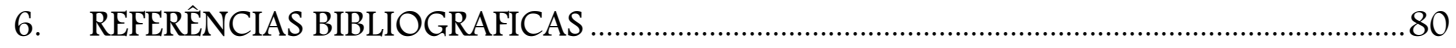

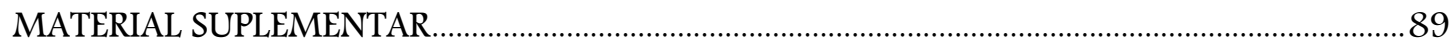

CAPÍTULO 3: ESPÉCIES AMEAÇADAS E ENDÊMICAS DO CERRADO E AS METAS PARA A CONVENÇÃO SOBRE DIVERSIDADE BIOLÓGICA - CDB ……………………………………........ 101

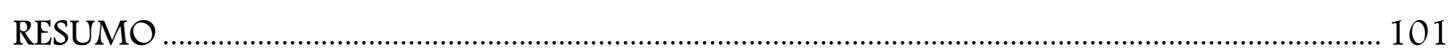

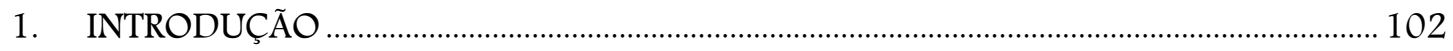

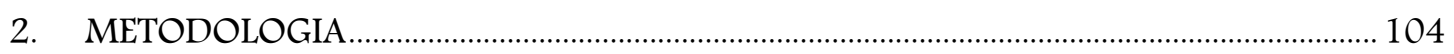

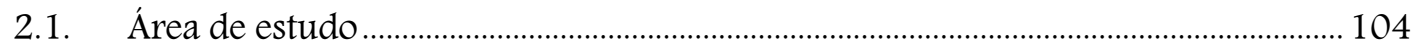

2.2. Índice da lista vermelha (Red List Index - RLI) ………………………………........ 106

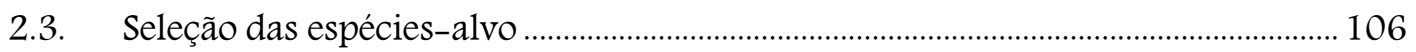

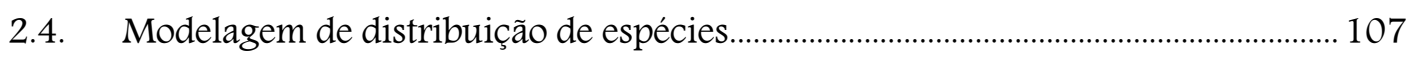

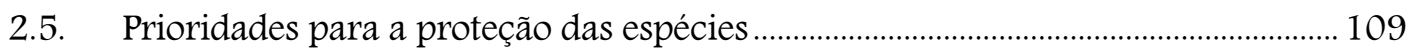

3. RESULTADOS

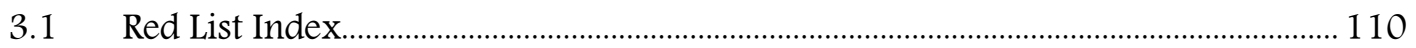

3.2. Modelos de distribuição potencial e avaliação da perda de área das espécies..... 112

3.3. REDES PRIORITÁRIAS PARA A PROTEÇÃO DAS ESPÉCIES-ALVO ………………….... 116

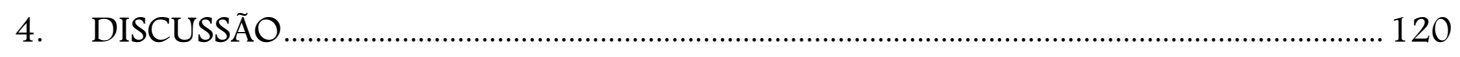

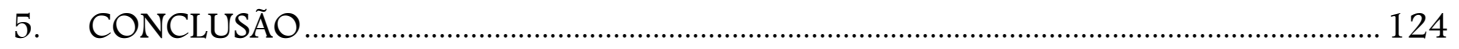

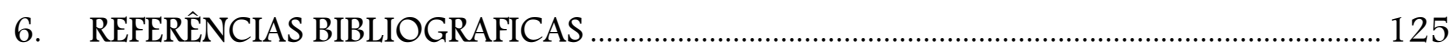

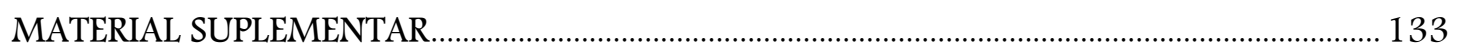

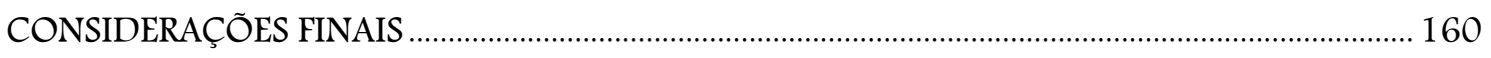

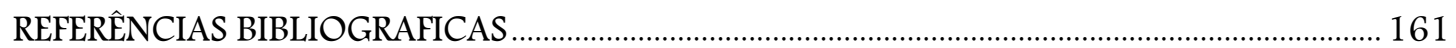




\section{RESUMO}

Desde a década de 70 existem iniciativas governamentais para o planejamento da conservação no Brasil que, inicialmente, eram baseadas somente no conhecimento ad hoc de pesquisadores e gestores. Contudo, a partir de 2006 o país passou a adotar uma metodologia denominada Planejamento Sistemático da Conservação (PSC) para a seleção de áreas prioritárias com o objetivo de conservação da biodiversidade. A utilização dessas metodologias está relacionada ao monitoramento das metas assumidas perante a Convenção sobre Diversidade Biológica (CDB), contudo, apesar do PSC requerer critérios explícitos e quantificáveis, os produtos advindos de sua utilização parecem não ser diretamente aplicados na gestão da conservação e avaliação das metas assumidas. Observa-se que áreas protegidas são criadas sob a óptica de critérios variados por vezes isolados, e o processo normalmente não leva em consideração as lacunas de conservação da biodiversidade existentes no sistema de unidades de conservação. Nesta pesquisa avaliamos como o PSC, especificamente seus princípios elementares (vulnerabilidade, insubstituibilidade e complementaridade), pode orientar o processo de tomada de decisão no cenário da conservação ambiental e na avaliação das metas estabelecidas no âmbito da CDB. Para isso utilizamos ferramentas adicionais de suporte à decisão: um algoritmo preditivo sobre a potencial distribuição das espécies, um modelo para a simulação da dinâmica de uso e ocupação do solo (LUCC) e programas que modelam a seleção de reservas. O uso combinado dessas ferramentas é a produção de mapas que priorizam áreas para a conservação da biodiversidade, os quais são essenciais para traçar cenários de conservação baseados nas propostas brasileiras para a CDB. A finalidade última é entender como as instituições brasileiras se apoderaram desta metodologia e como essas ferramentas podem auxiliar a gestão da nossa biodiversidade. O Cerrado foi utilizado como modelo para a aplicação dessas técnicas, já que é um bioma formado por um mosaico heterogêneo de ambientes savânicos e florestais, variando também nos aspectos físicos, como quanto ao histórico de ocupação antrópica de suas porções. Os modelos de LUCC indicaram que se forem mantidas as atuais tendências de desmatamento poderão ser observadas perdas de até $72 \%$ das áreas nativas em 2050 (cenário BAU). Em um cenário com governança, essas seriam menores, chegando a $61 \%$. Os cenários também revelaram que uma estratégia de proteção dos grandes remanescentes do norte do bioma, mas dissociada de ações de comando e controle, seria insuficiente para manter a representatividade dos alvos selecionados neste estudo. Metas uniformes de $17 \%$ seriam insuficientes para a representação das espécies endêmicas e ameaçadas, indicando a necessidade de conservação de uma parcela maior e mais regionalizada do Cerrado. 


\section{INTRODUÇÃO}

A Convenção sobre Diversidade Biológica (CDB) é um acordo entre nações aprovado em 1992, cujo objetivo central é a proteção da biodiversidade em todos os seus níveis de complexidade (Brasil, 2000). Nosso país foi um dos primeiros signatários desta Convenção, efetivamente promulgada pelo Decreto ${ }^{\text {o. }} 2519$ de 16/03/1998 (Brasil, 1998).

Em encontros periódicos, as Conferências das Partes (COPs), são elaboradas as Decisões, que estabelecem protocolos, programas e metas específicas para os países signatários (Prates \& Irving, 2015). A Comissão Nacional de Biodiversidade - CONABIO (Brasil, 2003) busca implementar e internalizar os objetivos da Convenção, estabelecendo metas nacionais por meio de suas Resoluções. A Resolução CONABIO n ${ }^{0} 3$ (Brasil, 2006), baseado nas metas globais formuladas pela Decisão VII/26 da COP 6, estabeleceu metas de conservação para o período de 2006 - 2010 e elas preveem, entre outras, a redução das taxas de desmatamento, a criação de áreas protegidas em regiões consideradas prioritárias para a conservação (Brasil, 2007; Brasil, 2007b), a diminuição do número de espécies ameaçadas e a proteção integral das mesmas por meio de unidades de conservação (UCs).

A COP-7/CDB recomendou que os países estabelecessem um sistema representativo de áreas protegidas, observando as lacunas de conservação existentes para a proteção de sua biodiversidade. Essa Decisão (VII/28) elencou critérios para a complementação do sistema: áreas com baixa fragmentação, e/ou sob forte ameaça, e/ou que assegurem a proteção de espécies em risco ou migratórias, e ainda, aquelas consideradas insubstituíveis. Esse documento também trouxe a necessidade de garantir a persistência de espécies e dos processos, ao ressaltar a importância da configuração espacial das áreas selecionadas (Ex. tamanho mínimo viável; integridade física) (CDB, 2004), adotadas formalmente pelo Brasil a partir da publicação da Deliberação CONABIO n ${ }^{\text {o. }} 39$ de 14 de Dezembro de 2005 (Brasil, 2005).

A partir da Resolução CONABIO n ${ }^{\text {o. }} 6$ (Brasil, 2013), o Brasil corroborou o Plano Estratégico para a Biodiversidade para o período de 2011 - 2020 definido na COP 10, em Aichi, no Japão. O compromisso assumido foi de aumentar o percentual de proteção dos ecossistemas terrestres de 10 (compromisso anterior) para 17\%. Mas diferente da Resolução anterior, onde a representação da biodiversidade deveria ser orientada pelas áreas prioritárias (Brasil, 2007), na reedição deste documento houve a omissão desta vinculação. $O$ texto anterior também deixava explícito a necessidade de garantir a representação das espécies ameaçadas pelo sistema de áreas protegidas.

O primeiro planejamento para a conservação no Brasil surgiu na década de 70 (Wetterberg et al., 1976) e utilizou a riqueza e o endemismo de plantas e animais, concentradas 
em regiões delimitadas pela teoria do refúgios, (Haffer, 1969; Prance, 1973; Vanzolini \& Williams, 1981) além de outros parâmetros ecológicos (ver Rylands, 1990; Rylands \& Brandon, 2005), para a seleção de áreas prioritárias na Amazônia. Em 1990, especialistas se reuniram em um Workshop para mais uma vez propor áreas prioritárias para a conservação na Amazônia, e a riqueza de espécies (sobretudo endêmicas) foi o principal critério de seleção de alvos entre os grupos temáticos formados (Rylands, 1990). Nesse momento, era compreensível que os processos de planejamento buscassem as áreas com maior riqueza de espécies, dado o baixo percentual de proteção por UCs (Figura 1) e a grande disponibilidade de territórios para esta finalidade.

Entretanto, o processo de priorização de áreas, antes focado na busca por áreas mais ricas, deu lugar à conservação das diferenças, a complementaridade (Vane-Wright et al., 1991) e a insubstituibilidade (Margules \& Pressey, 2000, Sarkar, 2012). A complementaridade, ou o grau em que uma região adiciona atributos ainda não contemplados pelas ações de conservação (McGeoch, 1998), busca a representação da biodiversidade, mas de forma a preencher as lacunas do já existente sistema de áreas protegidas. Associado a este conceito, a insubstituibilidade é uma medida atribuída a uma porção do território, que visa refletir a sua contribuição de uma porção de território para a região de estudo, de acordo com as metas quantitativas formuladas. Na prática é definido pela frequência de seleção dessas regiões durante os exercícios de priorização (Margules \& Pressey, 2000).

Os conceitos trazidos pela CDB: a complementaridade, a insubstituibilidade, a vulnerabilidade e a persistência, formam o escopo da metodologia denominada Planejamento Sistemático da Conservação - PSC (Margules \& Pressey, 2000). O PSC é uma ferramenta de suporte a decisão, que utiliza critérios explícitos, para a seleção de áreas. Há inúmeros algoritmos específicos desenvolvidos para essa finalidade (Sarkar, 2012), os quais permitem a manipulação de cenários de conservação atribuindo flexibilidade ao processo.

Uma série de etapas norteia a aplicação do PSC: compilação das informações biológicas, cartográficas e socioeconômicas da região de estudo; identificação dos objetivos da conservação; definição de alvos e metas quantitativas de conservação; definição das unidades de planejamento (UPs); revisão da representatividade ou análise de lacunas de conservação das áreas protegidas existentes; utilização de algoritmos para selecionar, desenhar e priorizar áreas adicionais para a conservação da biodiversidade; implementação de ações para a conservação; monitoramento e gestão adaptativa para garantir a manutenção da persistência e da funcionalidade da biodiversidade dentro das áreas selecionadas (Margules \& Pressey, 2000; Sarkar \& Illoldi-Rangel, 2010). 
Os primeiros planos para a conservação ainda não incorporavam explicitamente as pressões exercidas sobre o território, ou sua vulnerabilidade (Margules \& Pressey, 2000; Pressey \& Taffs, 2001; Sarkar et al., 2006; Linke et al., 2008) em geral, essa informação era agregada $a$ posteriori, de maneira ad hoc, e para estabelecer um ranking de prioridades de manejo das áreas. O PSC surgiu como uma ferramenta para aperfeiçoar as ações de conservação, que hoje estão sob um pano de fundo mais aguerrido entre a área ambiental e econômica, e precisam de soluções rápidas, eficientes, reproduzíveis e defensáveis (Knight \& Cowling, 2007). O conceito da vulnerabilidade pode respaldar a busca reativa (áreas com maior risco), por exemplo "Biodiversity hotsposts" (Myers et al., 2000) ou proativa (áreas prístinas), "High-biodiversity wilderness áreas" (Mittermeier et al., 2003) (ver Brooks et al., 2006).

As taxas de extinção de espécies são oriundas majoritariamente pela perda e fragmentação de hábitats causada principalmente pelo crescimento populacional, aumento da necessidade de insumos e da demanda de áreas para a expansão dos setores econômicos (Pimm et al., 2014; Dirzo et al., 2014). A compreensão desses fenômenos, ou seja, onde ocorrem e em quais taxas, pode auxiliar nos esforços de proteção da biodiversidade (Pimm et al., 2014). Os modelos quantitativos ecológicos e de uso da terra vêm sendo utilizados para avaliar o impacto das ações humanas sobre o território (De Souza \& De Marco Jr., 2014; Rosa et al., 2014) e a conservação da biodiversidade (Bird et al., 2012), sobretudo na Amazônia. No Cerrado, ainda há poucos estudos, mas Ferreira et al. (2012) e Salmona (2013) estimaram a perda de hábitats utilizando modelos preditivos de uso e ocupação do solo (LUCC) e Faleiro et al. (2013) utilizaram cenários futuros de LUCC e modelos preditivos da distribuição potencial de espécies (SDMs) para priorizar áreas para a conservação da biodiversidade. As estratégias para a conservação da biodiversidade devem considerar a expansão das ameaças e, ao mesmo tempo, eliminar as lacunas de proteção das espécies (Rodrigues et al., 2004).

O estabelecimento de objetivos quantificados para a proteção da biodiversidade sinaliza para a necessidade de produzir, organizar e disponibilizar informações sobre aquilo que se deseja conservar. O Brasil, lentamente, vem gerando indicadores a partir da organização de suas bases de dados. Um exemplo disso é a divulgação das séries temporais de dados sobre a ocorrência de desmatamento nos biomas brasileiros (Brasil, 2012). Tal acervo de informação, associado ao uso de avançado arcabouço computacional, pode auxiliar os planos de conservação regionais ou federais (Pimm et al., 2014). E ainda assistir na avaliação das atuais metas propostas perante à CDB e reformulá-las a partir de critérios técnicos e científicos (Pressey et al., 2007). Esse processo permite o repensar das estratégias de conservação implementadas até o momento e subsidiar diálogos futuros com os setores econômicos e sociais (Gibbs et al., 2015). 
Considerando o exposto, o objetivo geral do estudo foi avaliar se o compromisso assumido pelo Brasil perante a CDB garante a representatividade e persistência dos ecossistemas nativos e suas espécies endêmicas e ameaçadas. Para tanto, foram utilizados o planejamento da conservação, os modelos de distribuição de espécies e o uso e cobertura do solo como ferramentas para avaliar se o cumprimento das metas da Convenção é factível ou não no Cerrado. A tese está organizada em três capítulos, descritos a seguir.

CAPÍTULO 1- O desenvolvimento de metodologias sistemáticas para a conservação e os preceitos da CDB levaram o país a utilizar o conceito de complementaridade para incrementar o já estabelecido sistema de unidades de conservação (Brasil, 2000b). Nossa hipótese foi que o aumento da complexidade metodológica em priorização de áreas, advindos da adoção do PSC, tem se traduzido em ações mais eficientes para a conservação da biodiversidade. Nossos objetivos foram: investigar como o processo de gestão da conservação incorporou os conhecimentos elaborados por essa ciência. Além disso, avaliamos quais foram as tendências da pesquisa e da prática em priorização de áreas no Brasil e a concordância espacial na criação de áreas protegidas com as áreas consideradas complementares ao sistema. O capítulo foi redigido em inglês e na forma de um manuscrito, pois foi submetido para a revista Natureza \& Conservação (Qualis B2).

Nos CAPÍTULOS 2 e 3 tratamos da avaliação do cumprimento das metas quando a dinâmica de desmatamento do Cerrado e as lacunas de conservação de vertebrados terrestres são levadas em consideração. Nossa hipótese foi que a aplicação das metas assumidas pelo Brasil perante a CDB não serão suficientes para alterar o modelo atual de uso e ocupação do solo e tampouco garantir a persistência e representatividade da biodiversidade. Como premissa, adotamos que os cenários futuros de "o mesmo de sempre" (Business as usual - BAU) apresentarão grande perda de área natural, continuando a diminuir em extensão, integridade e representatividade de espécies e ecossistêmica, apesar de alguns sucessos na diminuição do ritmo da destruição como um todo.

Desenvolvemos nossas pesquisas no bioma Cerrado, que possui grande heterogeneidade espacial de sua biodiversidade (Ratter \& Dargie 1992; Silva et al., 2006; Brannstrom et al., 2008) e discrepantes modelos de ocupação antrópica em suas diferentes porções (Machado et al, 2004; Brannstrom et al., 2008; Jepson et al., 2005). O bioma possui altas taxas de desmatamento (7.637 $\mathrm{Km}^{2}$ ano $^{-1}$ em 2011) e somente $50 \%$ de sua cobertura vegetal ainda persiste.

Assim, no CAPÍTULO 2 construímos cenários de ocupação futura do Cerrado: "Business as usual" (BAU), que mantém as atuais tendências de desflorestamento e outros de "Governança ambiental" (GOV) baseados em metas estipuladas pelo Brasil para a CDB (Brasil, 2006). Com isto, avaliamos a evolução da supressão dos remanescentes do Cerrado, das métricas da paisagem, 
da vulnerabilidade dos ecossistemas e a representatividade das classes de vegetação (Brasil, 1993 \& Brasil, 2010).

No CAPÍTULO 3 calculamos: o Red List Index (RLI) (Butchart et al., 2007) por classe de vertebrados, entre os anos de 2003 e 2014, com o objetivo de avaliar a evolução do status de conservação dessas espécies. De certa forma, o índice de lista vermelha avalia como tem ocorrido o sucesso (ou o fracasso) na conservação das espécies consideradas ameaçadas de extinção ao longo do tempo. Como premissa de que a perda de hábitats é a principal causa de extinção das espécies, selecionamos espécies de vertebrados terrestres endêmicos e ameaçados do Cerrado e elaboramos seus modelos de distribuição (SDMs), a seguir confrontamos com os modelos futuros de LUCC, cenários BAU e GOV. Desenhamos redes de conservação, delimitando um subconjunto de áreas com alta prioridade para a conservação das espécies-alvo e medimos qual o percentual de áreas remanescentes futuras seria suficiente para representar essas espécies.

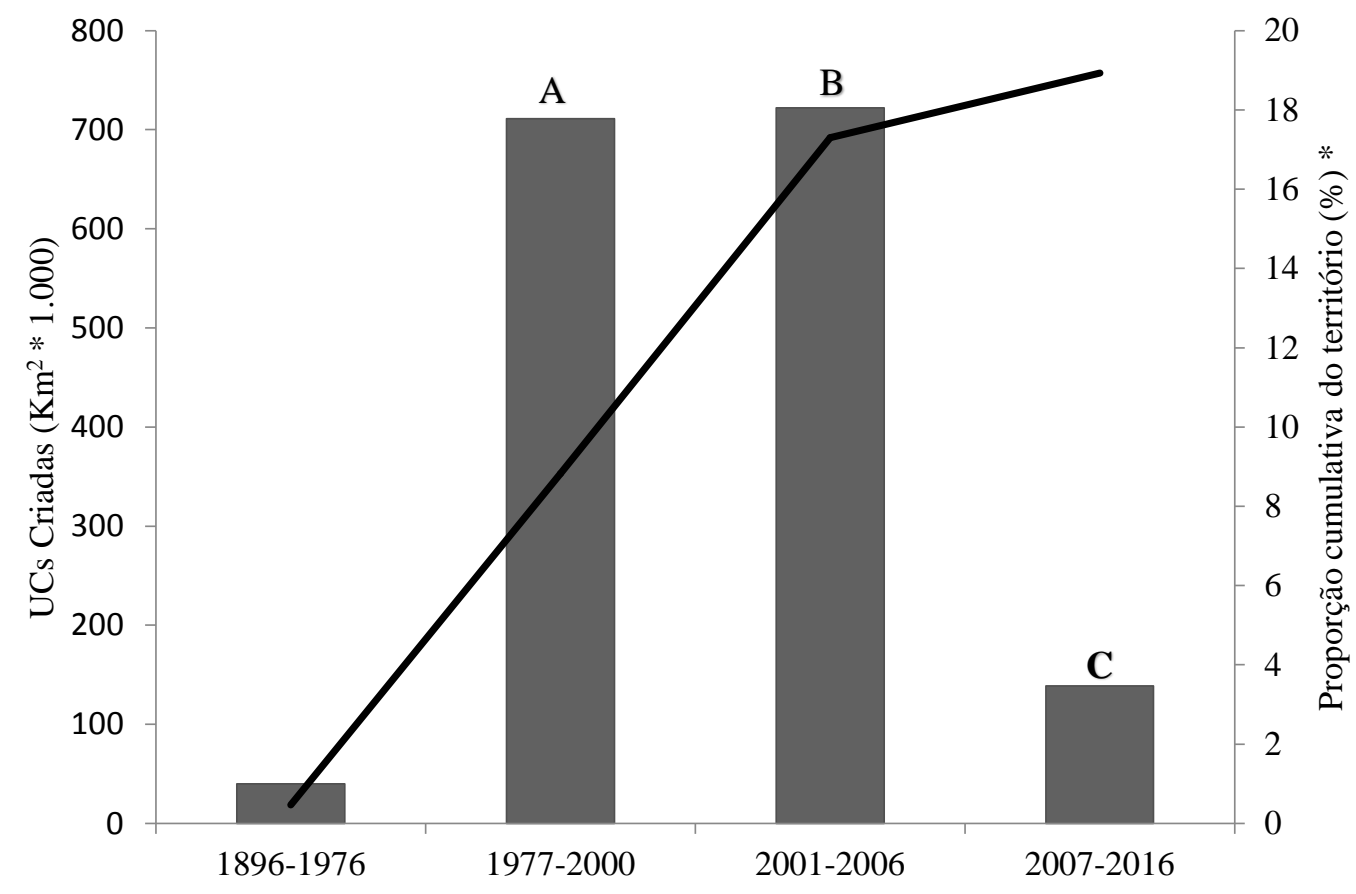

Figura 1: Criação de UCs (Brasil, 2016) agrupadas de acordo com os exercícios federais sobre planejamento da conservação no Brasil - A, após 1976: Wetterberg, 1976 \& Rylands, 1990 (487.252,50 Km² criados na Amazônia); B, após 2000: Brasil, 2004 e C, após 2006: Brasil, 2007. *Há sobreposições entre as UCs. 


\section{REFERÊNCIAS BIBLIOGRÁFICAS}

Bird, J.P., Buchanan, G.M., Lees, A.C., Clay, R.P., Develey, P.F., Yépez, I., Butchart, S.H.M., 2012. Integrating spatially explicit habitat projections into extinction risk assessments: a reassessment of Amazonian avifauna incorporating projected deforestation. Divers. Distrib. 18, 273-281. doi:10.1111/j.1472-4642.2011.00843.x.

Brannstrom, C., Jepson, W., Filippi, A.M., Redo, D., Xu, Z., Ganesh, S., 2008. Land change in the Brazilian Savanna (Cerrado), 1986-2002: Comparative analysis and implications for land-use policy. Land use policy 25, 579-595. doi:10.1016/j.landusepol.2007.11.008.

Brasil, 1993. Mapa de Vegetação do Brasil (1:5.000.000). Rio de Janeiro: IBGE.

Brasil, 1998. Decreto $n^{\text {o. }} 2519$ de 16/03/1998.

Brasil, 2000. A Convenção sobre Diversidade Biológica - CDB. Série Biodiversidade: Ministério do Meio Ambiente (MMA). 30p.

Brasil, 2000b. Lei ${ }^{\text {o. }} 9.985$, de 18 de Julho de 2000.

Brasil,2003. Decreto $n^{\circ} 4.703$, de 21 de Maio de 2003.

Brasil, 2004. Portaria MMA n ${ }^{\text {o. }}$ 126, de 27 de MAIO de 2004.

Brasil, 2005. CONABIO nº 39, de 14 de dezembro de 2005.

Brasil, 2006. Resolução CONABIO no 03, de 21 de Dezembro de 2006.

Brasil, 2007. Portaria MMA n ${ }^{\text {. }}$ 09, de 23 de janeiro de 2007.

Brasil, 2010. Ministério da Ciência e Tecnologia. Coordenação-Geral de Mudanças Globais do Clima. Segunda Comunicação Nacional do Brasil à Convenção-Quadro das Nações Unidas sobre Mudança do Clima. Brasília: Ministério da Ciência e Tecnologia. 2v. il.col.map.; 30cm. $+\mathrm{CD}-\mathrm{ROM}\left(4^{3 / 4} \mathrm{in}\right.$.)

Brasil, 2012. Monitoramento do bioma Cerrado 2002 a 2008. Monitoramento do desmatamento nos biomas brasileiros por satélite: acordo de cooperação técnica MMA/IBAMA. Relatório técnico, Instituto Brasileiro do Meio Ambiente e dos Recursos Naturais Renováveis. URL http://www.mma.gov.br/portalbio.

Brasil, 2013. Resolução CONABIO nº 06, de 3 de Setembro de 2013.

Brasil, 2016. Base digital de dados geoespacializados das áreas protegidas do Brasil, compilados pelo IBAMA, com dados do Cadastro Nacional de Unidades de Conservação e órgãos estaduais e municipais de meio ambiente (atualizado até 08/03/2016).

Brooks, T.M., Mittermeier, R. A, da Fonseca, G. A B., Gerlach, J., Hoffmann, M., Lamoreux, J.F., Mittermeier, C.G., Pilgrim, J.D., Rodrigues, A S.L., 2006. Global biodiversity conservation priorities. Science. 313, 58-61. doi:10.1126/science.1127609

Butchart, S.H.M., Akçakaya, H.R., Chanson, J., Baillie, J.E.M., Collen, B., Quader, S., Turner, W.R., Amin, R., Stuart, S.N., Hilton-Taylor, C., 2007. Improvements to the Red List Index. PLoS One 2. doi:10.1371/journal.pone.0000140 
CDB, 2004. Disponível em https://www.cbd.int/decisions/cop/?m=cop-07

De Souza, R.A., De Marco, P., 2014. The use of species distribution models to predict the spatial distribution of deforestation in the western Brazilian Amazon. Ecol. Modell. 291, 250-259. doi:10.1016/j.ecolmodel.2014.07.007

Dirzo, R., Young, H.S., Galetti, M., Ceballos, G., Isaac, N.J.B., Collen, B., 2014. Defaunation in the Anthropocene. Science 345, 401-406. doi:10.1126/science.1251817

Faleiro, F. V., Machado, R.B., Loyola, R.D., 2013. Defining spatial conservation priorities in the face of land-use and climate change. Biol. Conserv. 158, 248-257. doi:10.1016/j.biocon.2012.09.020

Ferreira, M.E., Ferreira, L.G., Miziara, F., Soares-Filho, B.S., 2012. Modeling landscape dynamics in the central Brazilian savanna biome: future scenarios and perspectives for conservation. J. Land Use Sci: 8, 1-19. doi:10.1080/1747423X.2012.675363

Gibbs, B.H.K., Rausch, L., Munger, J., Schelly, I., Morton, D.C., Noojipady, P., Barreto, P., Micol, L., Walker, N.F., Amazon, B., Cerrado, E., 2015. Brazil's Soy Moratorium. Science (80-. ). 347, 377-378. doi:10.1126/science.aaa0181

Haffer, J, 1969. Speciation in Amazonian Forest Birds. Science (165). 3889, 1969. http://doi.org/10.1126/science.165.3889.131

Jepson, W., 2005. A disappearing biome? Reconsidering land-cover change in the Brazilian savanna. Geogr. J. 171, 99-111. doi:10.1111/j.1475-4959.2005.00153.x

Knight, A. T., \& Cowling, R. M., 2007. Embracing opportunism in the selection of priority conservation areas. Conservation Biology: The Journal of the Society for Conservation Biology, 21(4), 1124-6. http://doi.org/10.1111/j.1523-1739.2007.00690.x

Linke, S., Norris, R.H., Pressey, R.L., 2008. Irreplaceability of river networks: towards catchment-based conservation planning. J. Appl. Ecol. 45, 1486-1495. doi:10.1111/j.13652664.2008.01520.x

Machado, R.B., M.B. Ramos Neto, P.G.P. Pereira, E.F. Caldas, D.A. Gonçalves, N.S. Santos, K. Tabor e M. Steininger, 2004. Estimativas de perda da área do Cerrado brasileiro. Relatório técnico não publicado. Conservação Internacional, Brasília, DF.

Margules, C.R., Pressey, R.L., 2000. Systematic conservation planning. Nature 405, 243-53. doi:10.1038/35012251

McGeoch, M. A.,1998. The selection, testing and application of terrestrial insects as bioindicators. Biological Reviews of the Cambridge Philosophical Society, 73(2), 181-201. http://doi.org/10.1017/S000632319700515X

Mittermeier, R. A., Mittermeier, C.G., Brooks, T.M., Pilgrim, J.D., Konstant, W.R., da Fonseca, G. A. B., Kormos, C., 2003. Wilderness and biodiversity conservation. Proc. Natl. Acad. Sci. U. S. A. 100, 10309-13. doi:10.1073/pnas.1732458100

Myers, N., Mittermeier, R. a, Mittermeier, C.G., da Fonseca, G. a, Kent, J., 2000. Biodiversity hotspots for conservation priorities. Nature 403, 853-8. doi:10.1038/35002501 
Pimm, S.L., Jenkins, C.N., Abell, R., Brooks, T.M., Gittleman, J.L., Joppa, L.N., Raven, P.H., Roberts, C.M., Sexton, J.O., 2014. The biodiversity of species and their rates of extinction, distribution, and protection. Science 344, 1246752. doi:10.1126/science.1246752

Prance, G. T., 1973. Phytogeographic support for the theory of Pleistocene forest refuges in the Amazon basin, based on evidence from distribution patterns in Caryocaraceae, Chrysobalanaceae, Dichapetalaceae and Lecythidaceae. Acta Amazonica 3:5-28.

Prates, A.P.L. \& Irving, M. d. A., 2015. Conservação da Biodiversidade e Políticas Públicas para as Áreas Protegidas no Brasil: Desafios e Tendências da Origem da CDB às Metas de Aichi. Rev. Bras. Politicas Públicas 1, 28-58. doi:10.1017/CBO9781107415324.004

Pressey, R.L.;Taffs, K.H., 2001. Scheduling priority conservation action in production landscapes: priority areas in western New South Wales defined by irreplaceability and vulnerability to vegetation loss. Biological Conservation 100, 345-376.

Pressey, R.L., Cabeza, M., Watts, M.E., Cowling, R.M., Wilson, K. A., 2007. Conservation planning in a changing world. Trends Ecol. Evol. 22, 583-92. doi:10.1016/j.tree.2007.10.001.

Ratter J.A., \& Dargie T.C.D., 1992. An analysis of the floristic composition of 26 cerrado areas in Brazil. Edinburgh Journal of Botany 49, 235-250.

Rodrigues, A.S.L., Andelman, S.J., Bakarr, M.I., Boitani, L., Brooks, T.M., Cowling, R.M., Fishpool, L.D.C., Fonseca, G.A.B., Gaston, K.J., Hoffmann, M., Long, J.S., Marquet, P.A., Pilgrim, J.D., Pressey, R.L., Schipper, J., Sechrest, W., Stuart, S.N., Underhill, L.G., Waller, R.W., Watts, M.E.J., Yan, X., Animale, B., 2004. Effectiveness of the global protected area network in representing species diversity. Nature 428, 9-12. doi:10.1038/nature02459.1

Rosa, I.M.D., Purves, D., Carreiras, J.M.B., Ewers, R.M., 2014. Modelling land cover change in the Brazilian Amazon: temporal changes in drivers and calibration issues. Reg. Environ. Chang. 1-30. doi:10.1007/s10113-014-0614-z

Rylands, A.B., 1990. Priority areas for conservation in the Amazon. Trends Ecol. Evol. 5, 240241.

Rylands, A. B., \& Brandon, K., 2005. Brazilian Protected Areas. Conservation Biology, 19(3), 612-618. http://doi.org/10.1111/j.1523-1739.2005.00711.x

Salmona, Y.B., 2013. Cerrado com C ou com S? Modelagem de Cenários Futuros para o Bioma. Dissertação de Mestrado, Universidade de Brasília (DF), 87p.

Sarkar, S., Pressey, R.L., Faith, D.P., Margules, C.R., Fuller, T., Stoms, D.M., Moffett, A., Wilson, K. a., Williams, K.J., Williams, P.H., Andelman, S., 2006. Biodiversity Conservation Planning Tools: Present Status and Challenges for the Future. Annu. Rev. Environ. Resour. 31, 123-159. doi:10.1146/annurev.energy.31.042606.085844

Sarkar, S., \& Illoldi-Rangel, P., 2010. Systematic Conservation Planning: an Updated Protocol. Natureza \& Conservação, 08(01), 19-26. http://doi.org/10.4322/natcon.00801003

Sarkar, S., 2012. Complementarity and the selection of nature reserves : algorithms and the origins of conservation planning,. Arch.Hist.Exact.Sci., 397-426. http://doi.org/10.1007/s00407012-0097-6 
De Souza, R.A., De Marco, P., 2014. The use of species distribution models to predict the spatial distribution of deforestation in the western Brazilian Amazon. Ecol. Modell. 291, 250-259. doi:10.1016/j.ecolmodel.2014.07.007

Silva, J. F.; Farinãs, M. R.; Felfili, J. M. \& Klink, C. A., 2006. Spatial heterogeneity, land use and conservation in the Cerrado region of Brazil. Journal of biogeography, 33, 536-548.

Wetterberg, G.B., Padua, M.T.J., de Castro, C.S. and de Vasconcellos, J.M.C., 1976. Uma Análise de Prioridades da Conservação da Natureza na Amazônia. Proj. Desenvolv. e Pesqui. Florest. UNDP/FAO/IBDF/ BRA-45. Ser. Tec. 8 .

Vane-Wright, R.I., Humphries, C.J., Williams, P.H., 1991. What to protect?-Systematics and the agony of choice. Biol. Conserv. 55, 235-254. doi:10.1016/0006-3207(91)90030-D

Vanzolini, P.E. \& Williams, E.E. 1981. Vanishing refuge: a mechanism for ecogeographic speciation. Papéis avulsos de Zoologia, 34, (23): 251-255. 


\section{CAPÍTULO 1}

\section{THE SCIENCE AND PRACTICE OF CONSERVATION PLANNING IN BRAZIL ${ }^{1}$}

\section{RESUMO EXPANDIDO}

\section{A CIÊNCIA E A PRÁTICA DO PLANEJAMENTO DA CONSERVAÇÃO NO BRASIL.}

A criação de áreas protegidas destaca-se entre as principais estratégias para a conservação da biodiversidade, tanto no Brasil quanto nos demais países (Prendergast et al., 1999; Margules \& Pressey, 2000). Contudo, a seleção dessas áreas é, em geral, oportunística (Pressey et al., 1993) e muitas vezes sua criação desvia-se dos objetivos centrais da conservação (Rylands \& Brandon, 2005; Margules \& Pressey, 2000; Margules et al., 2002). A ciência e a prática, envolvendo o planejamento da conservação e sua recente aplicação sistemática no Brasil, têm um desenvolvimento em paralelo assim, especulamos até que ponto o processo de gestão da conservação incorporou os conhecimentos elaborados por essa ciência. Nossa hipótese é de que o aumento da complexidade metodológica em priorização de áreas, advindos da adoção do Planejamento da Conservação, tem se traduzido em ações mais eficientes para a proteção das lacunas (Jennings, 2000) de representação da biodiversidade do sistema de áreas protegidas. A partir daí, primeiro, traçamos um panorama histórico sobre a ciência e a prática em priorização de áreas no Brasil, com o objetivo de apontar as tendências sobre o tema; em seguida, verificamos congruências relativas aos alvos de conservação, entre a ciência e a prática da conservação. Teorizamos que dois paradigmas alteraram a seleção de áreas no Brasil: a adoção dos conceitos de representatividade e de complementaridade. A representatividade pressupõe a conservação da biodiversidade em seus múltiplos níveis de organização (Noss, 1990). Para resguardar lacunas de representação nos sistemas de áreas protegidas, de uma determinada região, são empreendidos esforços para complementá-lo (Vane-Wright et al., 1991). Assim, áreas diferentes entre si, se tornam mais importantes ou insubstituíveis que as áreas mais ricas, se estas últimas forem redundantes neste sistema (Margules \& Pressey, 2000). No início, os planos buscavam áreas com alta riqueza de espécies e depois, o conceito de complementaridade foi utilizado para a priorização de áreas. Observamos vieses tanto na ciência como na prática (Duelli \& Obrist, 2003), tais como como o uso de espécies animais vertebrados, das medidas tradicionais de riqueza: concentração total de espécies, endêmicas e ameaçadas de extinção, e na localização das pesquisas científicas

1 Capítulo regidido em inglês e na forma de um manuscrito e, submetido para a revista Natureza \& Conservação (Qualis B2), autores: Giovana Bottura; Ludmilla Moura de Souza Aguiar \& Ricardo Bomfim Machado 
e exercícios práticos. A maioria dos exercícios práticos usou uma "cesta de indicadores", que em geral combina: espécies e atributos do ambiente, com o objetivo de resguardar as possíveis lacunas de conservação, dado o pouco conhecimento empírico sobre a distribuição das espécies. Não encontramos nenhum estudo que aborde as etapas do PSC referentes à implementação e ao monitoramento das ações de conservação. Também, investigamos a criação de áreas protegidas em regiões consideradas prioritárias com o objetivo de avaliar se as instituições que fazem a gestão da biodiversidade no Brasil vêm aplicando o planejamento da conservação em suas ações práticas. Para o primeiro planejamento da conservação federal para o Cerrado, em 1998, 44,14\% do número de áreas (ou 370.836,18 $\mathrm{Km}^{2}$ ) tinham como recomendação principal a criação de unidades de conservação. Entre as UCs criadas no período de 1999 a 2006 (142 UCs ou $\left.92.879,35 \mathrm{~km}^{2}\right) 31 \%(\mathrm{~N}=44)$ se sobrepõem aos polígonos prioritários para a criação de UCs, e $27 \%(\mathrm{~N}=16)$ se sobrepõem às áreas com recomendação para criação de Unidades de Conservação de Proteção Integral (UCPIs). Para o segundo exercício, realizado em 2006, foram sugeridas que 47,58\% das áreas priorizadas fossem reservadas à criação de UCs. Nesse período, de 2007 a 2016 foram criadas 42 UCs $\left(10.253,75 \mathrm{~km}^{2}\right)$ e, destas $62 \%(\mathrm{~N}=26)$ se sobrepõem aos polígonos prioritários para a criação de UCs, mas apenas $7 \%(\mathrm{~N}=2)$ se sobrepõem às áreas com recomendação para criação de UCPIs. A congruência espacial entre as propostas de áreas prioritárias e a criação de UCs, revelou que a abordagem do PSC pode ter tornado o processo mais eficiente $\left(\mathrm{x}^{2}=11.867, \mathrm{df}=1, \mathrm{p}\right.$-value $\left.<0.001\right)$. Contudo, quando restringimos esse universo amostral à comparação entre a criação de UCPIs e as áreas prioritárias (com esta recomendação), não encontramos diferenças significativas $\left(x^{2}=3.6135, \mathrm{df}=1, \mathrm{p}\right.$-value>0.05). Além da representatividade, o PSC busca a persistência dos alvos para garantir a existência futura das regiões com alta importância biológica. Mostramos que há poucos artigos científicos $(13,2 \%)$ e projetos práticos $(7.1 \%)$ que analisaram a incidência de riscos futuros, como aqueles advindos das mudanças climáticas e da dinâmica de uso e ocupação do solo. O Planejamento Sistemático da Conservação é um tema relativamente novo, mas que vem crescendo dentro da comunidade científica, haja visto o aumento substancial de artigos compilados. Contudo, a prática da conservação no Brasil evidencia que as discussões teóricas ainda não foram plenamente incorporadas ao dia-a-dia da conservação, existindo uma defasagem entre o crescimento científico e a aplicação do PSC pelas agências responsáveis pela gestão da biodiversidade no mundo (Pinto $\&$ Bini, 2008; Prendergast et al, 1999; Pullin et al, 2004). Mas esses mesmo autores observaram que esse crescimento científico tem uma grande importância, na medida em que exerce pressão sobre a prática da conservação. Observamos, por fim, que o desenvolvimento da ciência e da prática da conservação no Brasil ocorreram em paralelo, mas assim como observado por Prendergast et al. (1999), a ciência elabora questões sobre a seleção de reservas que talvez nunca tenham realmente uma aplicação prática. Enquanto outras, provavelmente venham a ser 
operacionalizadas para solucionar os problemas da prática da conservação (Knight et al., 2008), objetivo final da Biologia da Conservação (Robinson, 2006). 


\begin{abstract}
The science and practice involving conservation planning and its recent systematic applications in Brazil, have a parallel development. Two paradigms have changed the creation of protected areas (PAs) in Brazil: the adoption of the concepts of representativeness and complementarity. Initially, the conservation practice looked for areas with high species richness and subsequently the concept of complementarity was used to identification of conservation gaps, driven by systematic conservation planning (SCP), the decision support algorithms and the Convention on Biological Diversity (CBD). Biases were observed in the planning process: the predominant use of species (mostly vertebrates); the location of expertise and studies in Brazilian Cerrado (Savanna) and Atlantic Forest biomes and the use of richness: overall species; endemic species and threatened species. Starting in 2006 in Brazil, the use of the SCP approach helped on the identification of priority areas for biodiversity conservation and many PAs were created inside the proposed regions. Our results show that there are many similarities between the practice and science of conservation in Brazil.
\end{abstract}

Keywords: CBD; conservation planning; Systematic Conservation Planning; reserve design; protected areas. 


\section{INTRODUCTION}

The Conservation Biology is an area of science that has emerged in order to understand the biodiversity crisis (Soulé, 1985). The creation of protected areas (PAs) stands out from the main strategies for biodiversity conservation in Brazil and worldwide (Margules \& Pressey, 2000). However, the creation process of these areas is generally opportunistic, mainly because they are established in regions without any immediate human interest, such as for agriculture purposes (Pressey et al., 1993). The policy for conservation set aside should consider the concept of complementarity (Vane-Wright et al., 1996), to include the conservation of unprotected species and ecosystems.

The conservation planning in Brazil began to be practiced in the 70s (Wetterberg et al., 1976; Rylands, 1990). The process means that important areas for biodiversity are defined previously to the creation of PAs. Some of the large parks and reserves created in Amazon in the 80's, such as Jaú National Park $\left(23,673 \mathrm{~km}^{2}\right)$ or Amazônia National Park $\left(10,848 \mathrm{~km}^{2}\right)$, followed the proposed Pleistocene centers of endemism (Silva, 1998). The priority areas have only been established as a management tool for biodiversity conservation at the federal level in 1998 (Brazil, 2004). These early approaches still contain major components of subjectivity (Machado et al., 2012, Sarkar, 2012). Thus, public and private institutions encouraged by Convention on Biological Diversity (CBD), and the scientific knowledge already accumulated on the subject in the country, have begun to incorporate, in their strategies for selecting areas, the Systematic Conservation Planning (SCP) (Margules \& Pressey, 2000). SCP provides a framework that combine scientific knowledge to the practice of conservation (Sarkar, 2012), and its scope includes the principles of conservation actions, complementarity, irreplaceability and vulnerability (Margules \& Pressey, 2000; Pressey \& Taffs, 2001). These concepts are operated by algorithms specifically designed for this purpose (Pressey et al., 1997; Sarkar, 2012).

The science and practice involving conservation planning and its recent systematic application in Brazil have a parallel development, thus we are interested in knowing how the conservation managing process incorporates the knowledge produced by science. According to Knight et al. (2008), there is a gap between knowledge and the performance of organizations that manage the conservation, and our hypothesis assumed that the use of SCP principles in Brazil produced more effective results to fill gaps in the conservation of biodiversity. To minimally understanding of this process, we first draw a historical overview of the science and practice in prioritization of areas in Brazil, aiming at to point out the trends and check their consensus. Finally, we investigated the creation of PAs in priority areas in order to assess whether the institutions that are responsible for biodiversity management in Brazil have applied conservation planning in their day-to-day actions. 


\section{MATERIAL AND METHODS}

We searched for papers (science) about the application of Conservation Planning (CP) and Systematic Conservation Planning (SCP) (for details see Table S1) in the following online databases: Web of Science's Institute for Scientific Information, ISI - Thomson Scientific, Philadelphia (http://apps.webofknowledge.com/), Scopus (http://www.scopus.com/ scopus/home.url) and Google Scholar (http://scholar.google.com.br). The timeframe covered publications from 1990 to August 2015. The keywords used in the search were "reserve*selection*"; "reserve*design*"; "Systematic conservation planning"; "priority*area* AND selection"; "reserve planning", "simulated annealing AND conservation", "irreplaceability" and all searches were refined for countries/ territories: Brazil. We also used keywords in Portuguese on the Google Scholar data base: "Planejamento Sistemático da Conservação"; "área*prioritária*AND seleção"; "insubstituibilidade"; "seleção de unidades de conservação"; "seleção de áreas protegidas"; "seleção AND prioridades AND conservação". To search for reports (practice) in CP and SCP in technical publications (the "gray literature"), we used institutional reports and others publications, consulted by web portal of governmental and non-governmental agencies (at regional, federal or local levels). Thus, we accessed the websites of the Brazilian Institute of Environment and Renewable Natural Resources - IBAMA; the Ministry of the Environment - MMA; World Wildlife Fund - WWF; Conservation International CI, and Biodiversitas Foundation. We selected papers aimed to discuss and evaluate the systematic selection process. Among the papers found, we consider those that contemplate the application of conservation planning in Brazil, and included national authorship in broader regions. We extracted the following information from scientific papers: (i) year; (ii) journals; (iii) language; (iv) biomes; (v) conservation features (or objects); (vi) conservation targets (or goals); (vii) vulnerability analysis; and from the gray literature: (i) year; (ii) biomes; (iii) conservation features; (iv) conservation targets; (v) vulnerability analysis. In addition, we assessed what percentage of these papers applied the SCP methodology.

We obtained data for federal, state or municipal PAs in the Brazilian Cerrado (Savanna), except Private Reserves of Natural Heritage (RPPNs) (Brasil, 2016). By using a geographic information system, we evaluated the spatial congruence between the PAs created in two periods, 1999-2006 and 2007-2016, with layers of conservation planning exercises in Cerrado from 1998 (Brasil, 2004) and 2006 (Brasil), respectively. We considered the priority areas with recommendation for creation or expansion of PAs and integral protected areas (IPs) (equivalent to the IUCN categories I to III). We calculated the number of PAs and strict PAs overlapping priority polygons, and tested whether the spatial confluence would be different from the chance by using a 2-sample proportion test (p-value <0.05). We used R 2.15.2 software to perform the statistical analysis. 
Concepts adopted in this paper: conservation planning (CP), driven by experts and, those from systematic methodology (SCP) (Margules \& Pressey, 2000) (Table S1).

\section{RESULTS}

We obtained a total of 93 scientific papers, 60 of those written in English and 33 in Portuguese. The papers are distributed in 43 different journals, being only six periodicals concentrated $51.61 \%$ of all publications (Fig. S2). Our results show a trend in the increasing of publications and reports related to CP or SCP in Brazil roughly from the year of 2002, with a peak in 2009 (Figs. 1A-B).

Around $41.18 \%$ and $23.52 \%$ of papers and $22.86 \%$ and $28.5 \%$ of the reports in conservation planning selected the Cerrado and the Atlantic Forest biomes as their research development sites, respectively. The number of features used on the analyzed PSC papers $(\mathrm{N}=60)$, showed a huge variation, ranging from 2004 to 2015 (mean $=472.67$, s.d. $=1376.8$ ) (Fig. S3A). A similar analysis that used 12 reports showed that the mean of feature was 1263.75 (s.d. $=2948.55$ ) (Fig. S3B).

Most papers (80.73\%) and reports (95.65\%) selected species among conservation features, and of these, $92.3 \%$ and $95.65 \%$ used vertebrate species group, respectively. Few papers used invertebrate species or plants (12.3\% and $16.92 \%$, respectively), corresponding to the opposite registered by reports, often of $73.91 \%$ and $65.22 \%$, respectively (Tables S4, S5). Mammals formed the most frequent group in papers and reports, followed by birds for the reports (Tables S4, S5, Fig. S6). Among the range of diversity measures, the most frequent parameter used in papers and reports was the species richness. Subdivisions of species included overall richness, number of endemic and threatened species (Fig. 2, Tables S4, S5). The SCP was applied in $81.72 \%$ of the papers and $53.85 \%$ of the reports. 

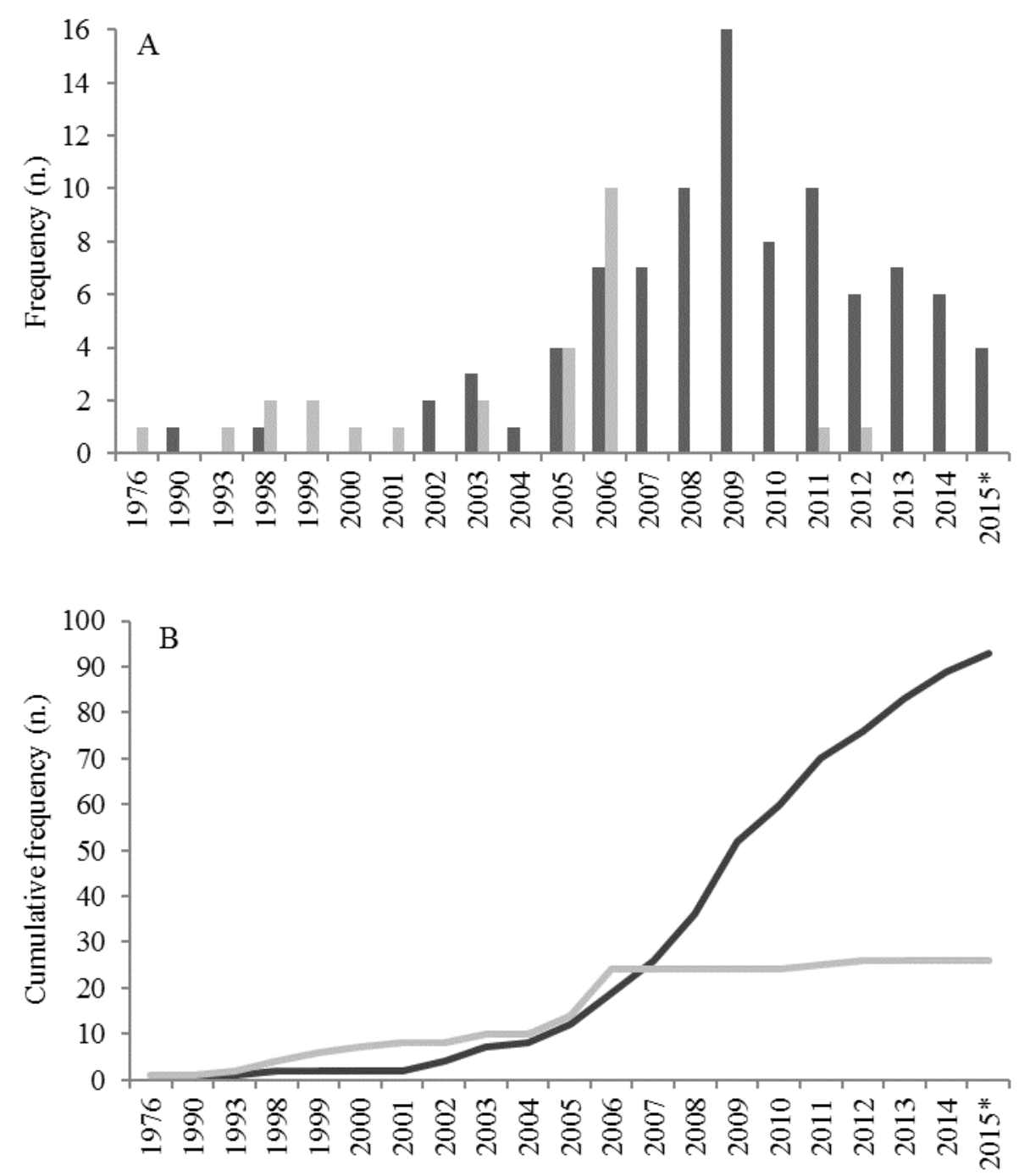

Figure 1: Temporal evolution of the frequency number (1A) and cumulative (1B) papers (dark gray) and reports (light gray). 


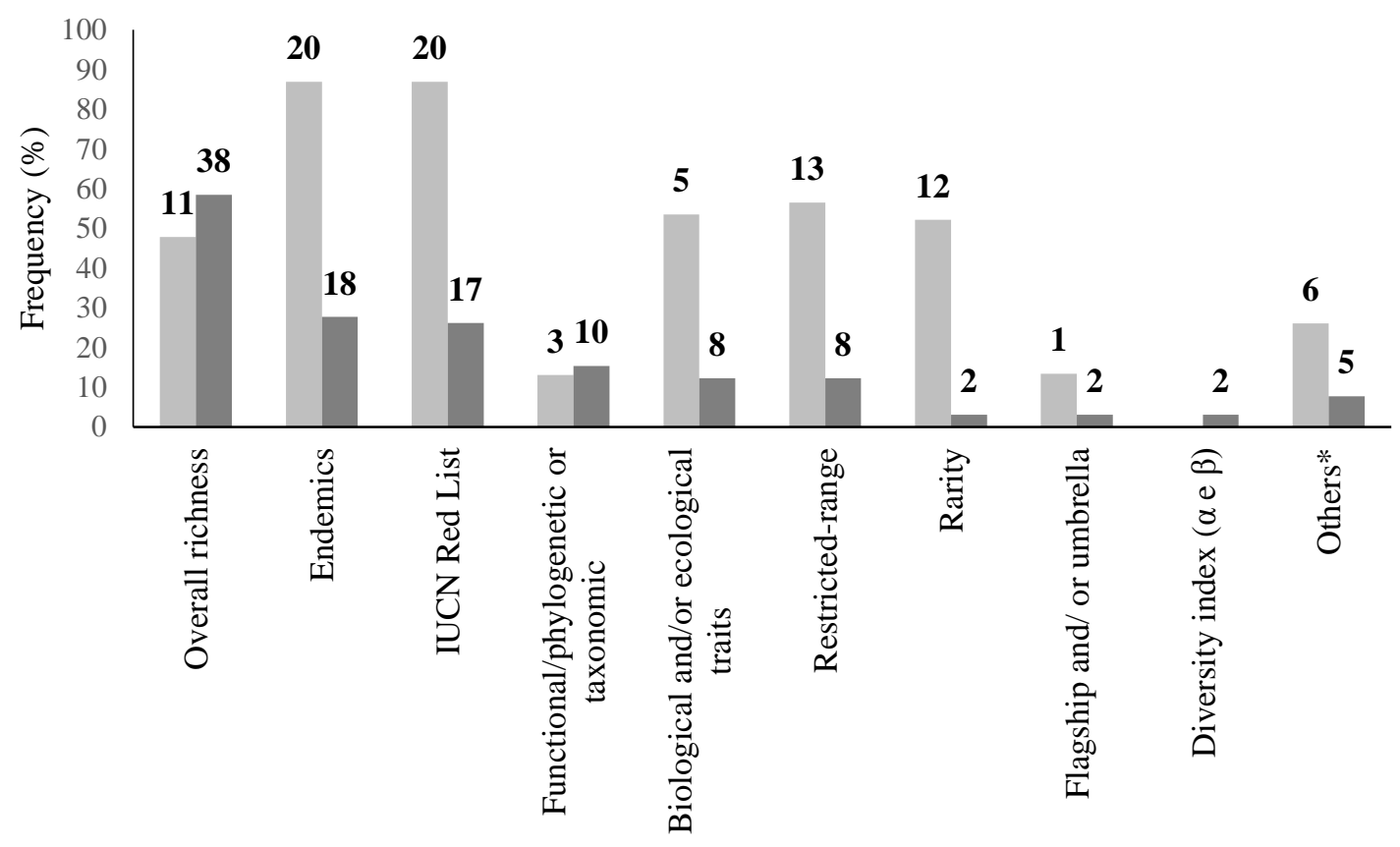

Figure 2: Frequency of diversity measures in papers (dark grey) and reports (light grey); absolute number of features (on the top) in Conservation Planning and Systematic Conservation Planning. * Others: species migratories, genetic diversity, biological and ecological features, temporal variability.

The practice initiatives for areas prioritization, at federal level can be divided into two groups according to their methodological guidelines: CP and SCP (Table S1). For the first exercise (1998), $44.14 \%$ of the areas (or $370,836.18 \mathrm{Km}^{2}$ ) had as main recommendation the creation of PAs. 31\% of PAs created between 1999 and $2006\left(\mathrm{~N}=142\right.$ or $\left.92,879.35 \mathrm{~km}^{2}\right)$ overlaps with priority polygons aiming at creation of PAs, and $27 \%(\mathrm{~N}=16)$ overlaps with polygons to creation of IPs (Fig. 3).

For the second assessment, conducted during 2006, 47.58\% of the priority areas should set aside for the creation of PAs. From 2007 to 2016, 42 PAs (or 10,253.75 km2) have been created, $62 \%(\mathrm{~N}=26)$ overlapping the priority polygons aiming at creation of PAs. Only $7 \%(\mathrm{~N}=2)$ of IPs overlap areas with similar goals (Fig. 4). 


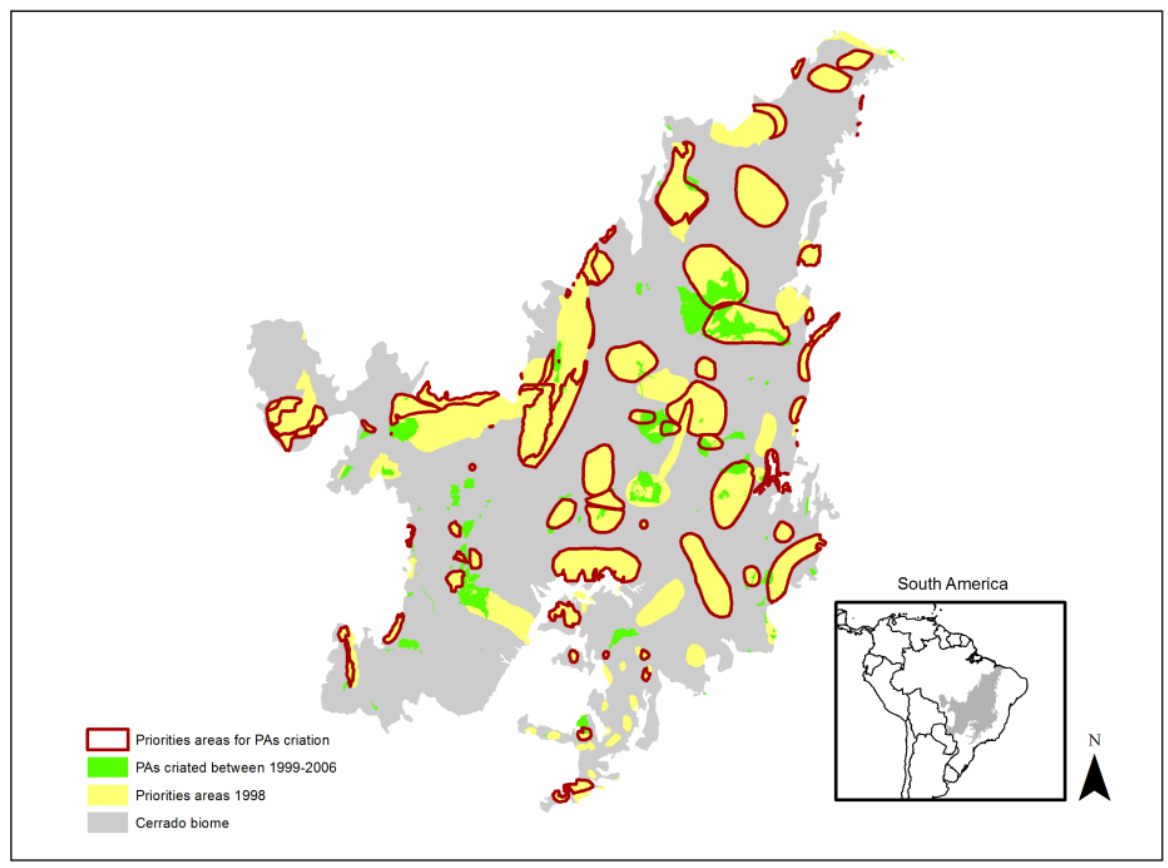

Figure 3: Overlap between priorities areas (from the first national exercise - 1998), and PAs proposed from 1999 to 2006

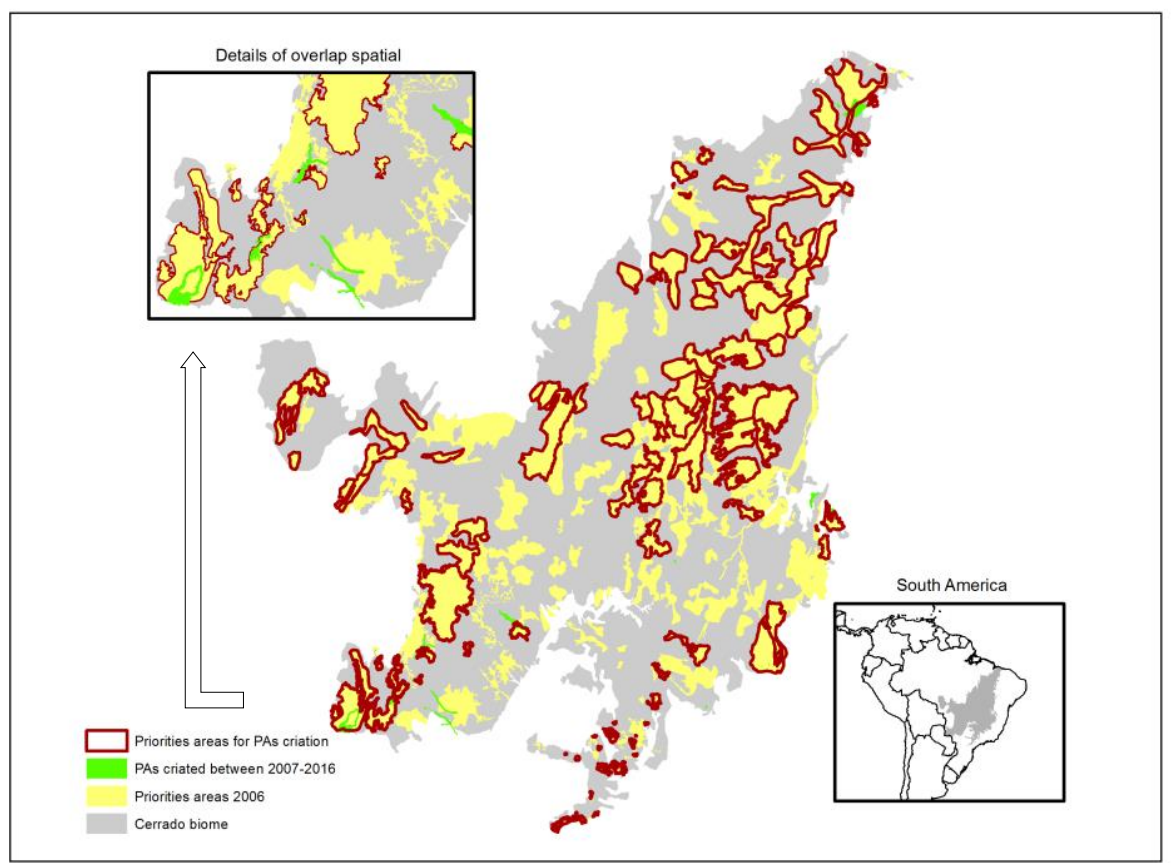

Figure 4: Overlap between priorities areas (from the second national exercise - 2006), and PAs proposed from 2007 to 2016.

Our analysis comparing the efficience of both assessments, measured as the spatial congruence between the proposed priority areas and the creation of new protected areas, revellead that more areas were created after applying the PSC approach than when tradicional assessement 
were done $\left(\mathrm{x}^{2}=11.867, \mathrm{df}=1, \mathrm{p}\right.$-value $\left.<0.001\right)$. However, we didn't find significant differences $\left(x^{2}=3.6135, \mathrm{df}=1, \mathrm{p}\right.$-value $\left.>0.05\right)$ between the IPs creation processes.

\section{DISCUSSION}

We saw two major paradigm that changed the science and practice of selection areas for conservation in Brazil, the adoption of the concepts of representativeness and complementarity. The first reported conservation planning in Brazil occurred in the Amazon biome (Wetterberg et al., 1976; Rylands, 1990), using parameters such as richness and endemism of plants and animals, to guide a national system of protected areas (Rylands, 1990, Rylands \& Brandon, 2005). PAs started been designed into their regional context, to promote a system of PAs, in order to safeguard the representation of biodiversity, the extent to which the pressures on the territory increased. This process was slow and not always widely used by state and federal managers (Rylands \& Brandon, 2005) and culminated with the regulations of the National Protected Areas System - SNUC (Brazil, 2000).

In the second change, the adoption of the SCP, which began in 2003, by non-governmental organization (NGOs) (WWF, 2005), as occurred in other countries (see Dowie, 2009). And, then by state and federal governments, supported by these early experiences, they began to embrace it within the scope of its management. There was the need to evaluate, monitor, and above all complement the SNUC, compared to commitments made at COP 7/ CBD.

Thus, the SCP has emerged as a tool to improve conservation actions, and need fast, efficient, repeatable and defensible solutions (Margules \& Pressey, 2000). The adoption of systematic selection brought clear advantages to the prioritization process areas, such as: (i) reducing subjectivity in the decision process (Cowling et al., 2003; Machado et al., 2012), (ii) the improved integration of data and different work schedules, (iii) support for the monitoring process, scenario development, that can help in decision-making on public policy and (iv) in the process of negotiation between the different actors of the Brazilian environmental panorama.

The use of areas with the highest species richness as the focus of conservation, was a concept that was replaced by the idea of complementarity, which emerged just with the basis of the SCP (Sarkar, 2012). Thus, the selection of priorities has become focused on the preservation of differences (Vane-Wright, 1996). Complementarity, searched to operationalize the concept of $\beta$ diversity, implemented by iteractive algorithms (Pressey et al. 1997; Sarkar, 2012).

We observed bias in papers, the use of species as surrogates of biodiversity, with taxonomic coverage based on animal species, particularly vertebrates (Pinto \& Grelle, 2009), which in general are the groups with greater availability of data (Diniz-Filho et al., 2009). In conservation 
practice most exercises used a basket of indicators, which, in general, combine: species and attributes of the environment, aiming at protecting potential conservation gaps.

The use of algorithms in the systematic selection enabled the increased complexity of ecological models, which have incorporated many of the theories and concepts developed by conservation biology. This increased complexity seeks to solve two problems in SCP: the representativeness and persistence (Margules \& Pressey, 2000; Gaston et al., 2002, Sarkar, 2012).

Traditional diversity indexes: such as overall species richness, of endemic and threatened species, is still being used as biodiversity substitutes. However, some papers have discussed others biodiversity indexes, such as, phylogenetic, functional, genetic, biological and ecological characteristics of species and ecosystems (Fig. 2, Tables S4, S5). The persistence can be ensured through targets and risks minimization. Most papers used uniform targets, for all features $(66,7 \%)$ : default or legally established thresholds (Tables S4, S5). The persistence concept can also be related to vulnerability of areas: by climate changes, by land use conflicts, or considering biological and ecological traits of species and its current conservation status. Most papers and reports consider only present threats. Few papers $(13.2 \%)$ and reports $(7.1 \%)$ used future (or dynamic) threats implemented by predictive models (Tables S4, S5) (Pressey et al., 2007). In practice, usually, the risks were assigned after the selection of the areas, of ad hoc way, driven the scheduling and execution of conservation priority action.

The results show that the prioritization exercises guided the selection of PAs, assisting in the design of regional strategies for biodiversity conservation, unlike observed by Margules et al. (2002). But the PAs creation process is governed by a series of social, political and economic factors, as well as biological and ecological approach, "informed opportunism" (Pressey \& Bottrill, 2008), where conservation actions are still governed by the opportunities of the real world, only now considering the priorities for biodiversity. The steps of monitoring and adaptive management regarding the SCP (Margules \& Pressey, 2000), are not incorporated by the practice of conservation in Brazil, and this is also a subject insufficiently researched in Brazil and in the world, is what Knight et al. (2008) named it the "crisis of implementation".

We note, finally, that the development of science and practice of conservation in Brazil took place in parallel, and expertise on the subject is concentrated in institutions in the Southeast and Center of the country (Pinto \& Grelle, 2009). This scientific background could elucidate the emphasis on practical experiences found in these biomes.

Most of the papers were published in the journal Nature \& Conservation that besides researchers, has among its target public environmentalists, technical and environmental area 
decision makers, suggesting a strong link between science and practice of conservation. Scientific growth is very important, in that it put pressure on the practice of conservation (Pullin et al., 2004). We observed a proportional development of science and the Conservation Planning practice, sometimes the practice driving the science, providing arguments for their researches and subsequently science, applying concepts and tools that supported the prioritization of areas in Brazil. 


\section{REFERENCES}

Brasil, 2000. Lei $\mathrm{n}^{0.9 .985}$, de 18 de Julho de 2000. Available at: http://www.planalto.gov.br/ccivil_03/leis/L9985.htm

Brasil, 2004. Portarias MMA $\mathrm{n}^{\mathrm{o}}$ 126, 27 de Maio de 2004. Available at: http://www.mma.gov.br/estruturas/chm/_arquivos/port126.pdf

Brasil, 2007. Portaria MMA $\mathrm{n}^{\circ}$ 9, de 23 de Janeiro de 2007. Available at: http://www.mma.gov.br/estruturas/chm/_arquivos/biodiversidade31.pdf

Brasil, 2016. Base digital de dados geoespacializados das áreas protegidas do Brasil, compilados pelo IBAMA, com dados do Cadastro Nacional de Unidades de Conservação e órgãos estaduais e municipais de meio ambiente (atualizado até 08/03/2016). Available at:

Cowling R et al., 2003. The expert or the algorithm? Comparison of priority conservation areas in the Cape Floristic Region identified by park managers and reserve selection software. Biological Conservation, 112:147-167.

Diniz-Filho JAF et al., 2009. Macroecologia, biogeografia e áreas prioritárias para conservação no Cerrado. Oecologia Brasiliensis, 13:470-497.

Dowie M, 2009. Conservation refugees: The hundred-year conflict between global conservation and native peoples. Cambridge: MIT Press.

Duelli, P; Obrist, M. K., 2003. Biodiversity indicators: the choice of values and measures. Agriculture, Ecosystems \& Environment, 98, 87-98. http://doi.org/10.1016/S01678809(03)00072-0

Gaston KJ et al., 2002.Persistence and vulnerability: retaining biodiversity in the landscape and in protected areas. Journal of Biosciences, 27: 361-84.

Jennings, M.D., 2000. Gap analysis: concepts, methods, and recent results. Landscape Ecology. 15 , p.5-20.

Knight AT et al., 2008. Knowing but not doing: selecting priority conservation areas and the research-implementation gap. Conservation biology, 22:610-617.

Machado RB et al., 2012. Os princípios da complementaridade e insubstituibilidade na revisão das áreas prioritárias para a conservação do Cerrado. In: Conservação da Biodiversidade com $S I G$, São Paulo: Oficina de Textos. p.226-240.

Margules CR \& Pressey RL, 2000. Systematic conservation planning. Nature, 405:243-53.

Margules CR, Pressey RL \& Williams PH, 2002. Representing biodiversity: data and procedures for identifying priority areas for conservation. Journal of Biosciences, 27: 309-26.

Noss RF, 1990. Indicators for Monitoring Biodiversity: A Hierarchical Approach. Conservation Biology, 4:355-364.

Pinto, Míriam Plaza; Bini, L.M., 2008. Vieses geográficas e taxonomicos nas pesquisas sobre seleção de reservas: uma análise quantitativa de 1992 a 2004. Nat. Conserv. 30, 322-326. doi:10.1590/S0100-29452008000200009 
Pinto MP \& Grelle CEV, 2009. Seleção de reservas: estudos na América do Sul e revisão de conceitos. Oecologia brasiliensis, 13:498-517.

Prendergast, J.R., Quinn, R.M., Lawton, J.H., 1999. The Gaps between Theory and Practice in Selecting Nature Reserves. Conserv. Biol. 13, 484-492. doi:10.1046/j.15231739.1999.97428.x

Pressey RL et al., 1993. Beyond opportunism: Key principles for systematic reserve selection. Trends in Ecology \& Evolution, 8: 124-128.

Pressey RL, Possingham HP \& Day JR, 1997. Effectiveness of alternative heuristic algorithms for identifying indicative minimum requirements for conservation reserves. Biological Conservation, 80: 207-219.

Pressey RL \& Taffs KH, 2001. Scheduling priority conservation action in production landscapes: priority areas in western New South Wales defined by irreplaceability and vulnerability to vegetation loss, Biological Conservation, 100:345-376.

Pressey, RL, Cabeza, M., Watts, ME, Cowling, R.M., Wilson, K. a, 2007. Conservation planning in a changing world. Trends Ecol. Evol. 22, 583-92. doi:10.1016/j.tree.2007.10.001

Pressey RL \& Bottrill MC, 2008. Conservation Biology, 22:1340-1345.

Pullin AS et al., 2004. Do conservation managers use scientific evidence to support their decisionmaking? Biological Conservation, 119: 245-252.

Robinson, J.G., 2006. Conservation Biology and Real-World Conservation. Conserv. Biol. 20, 658-669. doi:10.1111/j.1523-1739.2006.00469.x

Rylands AB, 1990. Priority areas for conservation in the Amazon. Trends in Ecology \& Evolution. 5: 240.

Rylands AB \& Brandon K, 2005. Brazilian Protected Areas. Conservation Biology, 19: 612-618.

Sarkar S, 2012. Complementarity and the selection of nature reserves: algorithms and the origins of conservation planning. Arch.Hist.Exact.Sci., 66: 397-426.

Silva JMC, 1998. Um método para o estabelecimento de áreas prioritárias para a conservação na Amazônia Legal. Relatório Técnico não publicado. WWF-Brasil. Brasília-DF. 18p.

Soulé ME, 1985. What is Conservation Biology? A new synthetic discipline addresses the dynamics and problems of perturbed species, communities, and ecosystems. BioScience, $35: 727-734$.

Wetterberg GB et al.,1976. Uma Análise de Prioridades da Conservação da Natureza na Amazônia, Projeto de Desenvolvimento e Pesquisa Florestal (PRODEPEF) UNDP/FAO/IBDF/ BRA-45. Série Técnica 8:63.

WWF, 2005. Visão da biodiversidade da ecorregião Serra do Mar, 170p.

Vane-Wright RI, 1996. Systematics and the conservation of biological diversity. Ann.Missouri Bot. Gard., 83: 47-57.. 


\section{SUPPLEMENTARY MATERIAL}

Table S1: Key differences between the processes of reserve seletion: systematic (SCP) and non-systematic (CP).

\begin{tabular}{|c|c|}
\hline Conservation Planning (CP) & Systematic Conservation Planning (SCP) \\
\hline $\begin{array}{l}\text { Use ad hoc knowledge on biodiversity to } \\
\text { select areas; } \\
\text { - Independent thematic proposals by features; } \\
\text { - Integration of information by spatial overlap } \\
\text { - without clear criteria; } \\
\text { - Representativess: driven by concepts of } \\
\text { richness. }\end{array}$ & $\begin{array}{l}\text { The ad hoc knowledge guides the features } \\
\text { (objects) and quantitative targets } \\
\text { development; } \\
\text { - Decision support software to design areas; } \\
\text { - Gap analysis; } \\
\text { - Representativeness: driven by concepts of } \\
\text { complementarity and irreplaceability; } \\
\text { - Persistence: driven by quantitative targets } \\
\text { and vulnerability (e.g. connectivity, risks); } \\
\text { - Implementation: opportunities costs can be } \\
\text { incorporated in area selection process; } \\
\text { - Flexibility: simulating the different } \\
\text { scenarios. }\end{array}$ \\
\hline
\end{tabular}




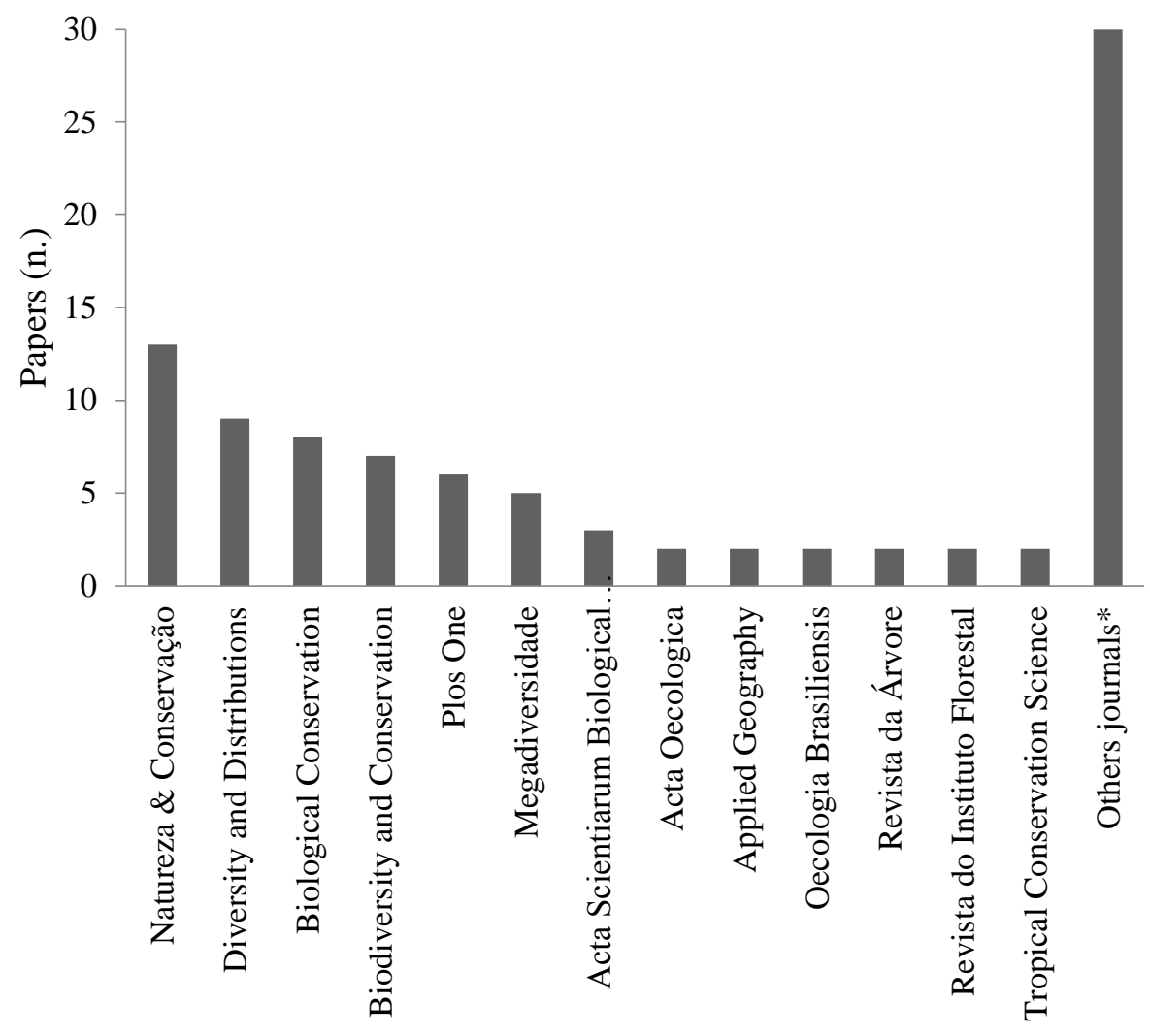

Figure S2: Number of papers published in specialized journals from 2004 to 2015. *Journals (N=1 paper): Acta Amazônica; Acta Geográfica; Amphibia-Reptilia; Applied Geography; Bioscience; Biotemas; Bird Conservation International; Boletim Goiano de Geografia; Brazilian Journal of Biology; Business; Conservation Biology; Conservation Ecology; Conservation Genetics; Ecology and evolution; Ecological Indicators; Environmental Conservation; Fragmentos de Cultura; Gaia Scientia; Genetics and Molecular Biology; Holos Environment, Iheringia, Série Zoológica; Journal of Biogeography; Journal for Nature Conservation; Lundiana; Mercator; Ocean \& Coastal Management; Oryx; Revista Brasileira de Cartografia; Revista de Biologia Neotropical; Tropical Conservation Science; Wetlands. 

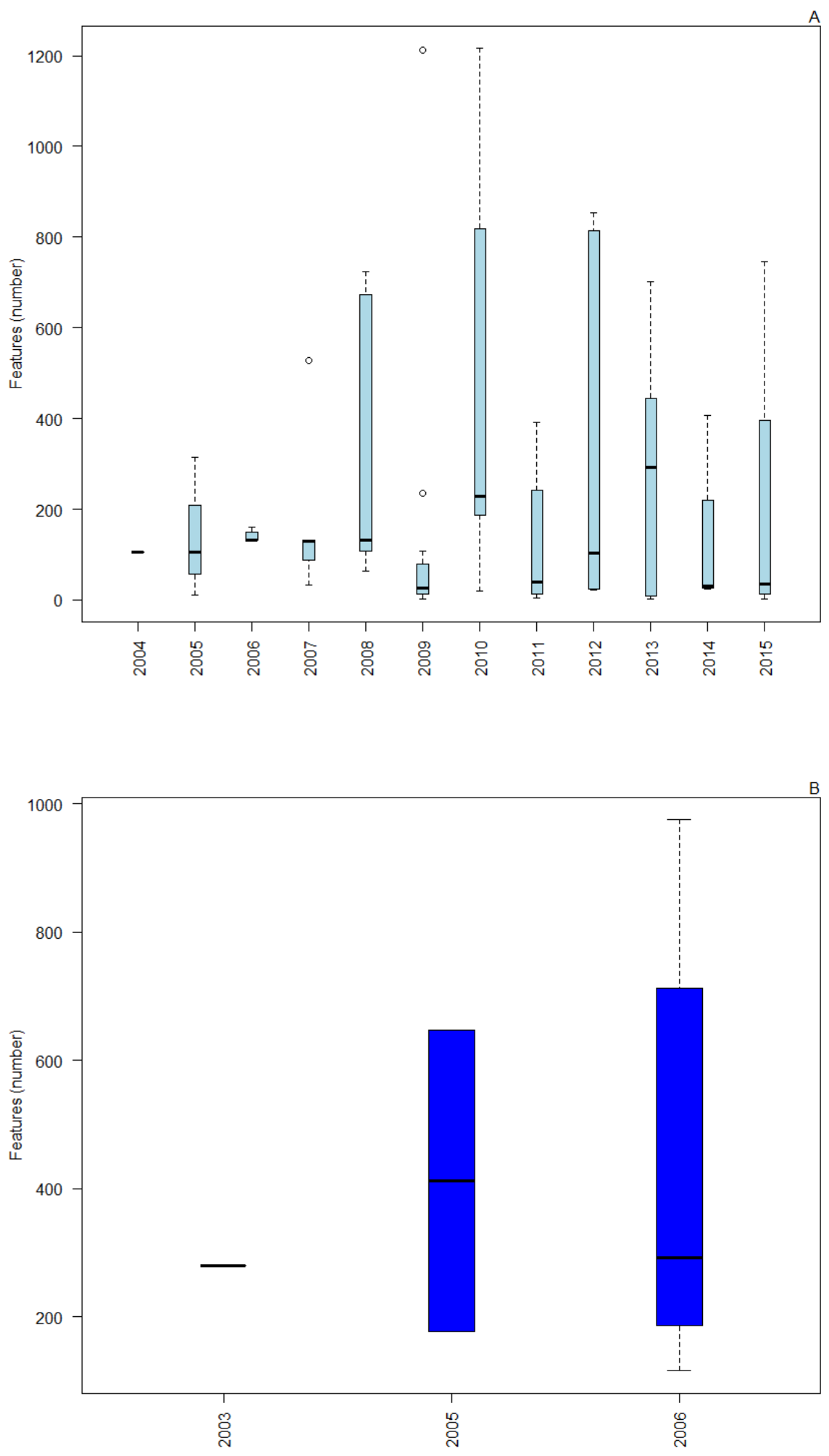

Figure S3: Number of features: A - in PSC papers/year, without outliers > 2000 features/paper (N=3) and, B - in PSC practice/year without outliers > 2000 features/reports $(\mathrm{N}=1)$. 
Table S4: The representativeness and persistence in the science. Features: types of species (V=vertebrates; I=invertebrates; P=plants), species diversity indexes, environmental (e.g. vegetation), environmental process (e.g. fragmentation, refuges). Targets: default threshold (uniform goals), political threshold (legally established goals), biological and ecological threshold (goals according to biological and ecological traits of features), minimum viable population size (Shaffer, 1981), conservation status and others. Threats: static (considering the current conditions) or dynamic (predictive models).

\begin{tabular}{|c|c|c|c|c|c|c|c|c|c|c|c|}
\hline \multicolumn{8}{|c|}{ REPRESENTATIVENESS } & \multirow{2}{*}{\multicolumn{3}{|c|}{ PERSISTENCE }} & \multirow{4}{*}{ REFERENCES } \\
\hline \multicolumn{8}{|c|}{ FEATURES } & & & & \\
\hline \multicolumn{5}{|c|}{ SPECIES } & \multirow{2}{*}{ ENV. } & \multirow{2}{*}{ ENV. PROCESS } & \multirow{2}{*}{ FEATURES $\mathbf{N}^{0}$} & \multirow{2}{*}{ TARGETS } & \multirow{2}{*}{$\begin{array}{c}\text { STATIC } \\
\text { THREATS }\end{array}$} & \multirow{2}{*}{$\begin{array}{l}\text { DYNAMIC } \\
\text { THREATS }\end{array}$} & \\
\hline YEAR & v. & I. & $\mathbf{P}$ & $\begin{array}{l}\text { SPECIES DIVEX } \\
\text { INDSIT }\end{array}$ & & & & & & & \\
\hline 1990 & $\checkmark$ & $\checkmark$ & $\checkmark$ & $\begin{array}{l}\text { Overall richness, } \\
\text { endemism }\end{array}$ & $\checkmark$ & $\checkmark$ & $\mathrm{ND}^{2}$ & & Land use & & $\begin{array}{l}\text { Rylands AB, 1990. Priority areas for conservation in the } \\
\text { Amazon. Trends in Ecology \& Evolution. } 5 \text { (8):240. }\end{array}$ \\
\hline 1998 & $\checkmark$ & $\checkmark$ & $\checkmark$ & $\begin{array}{l}\text { Overall richness, } \\
\text { endemism }\end{array}$ & & & ND & & & & $\begin{array}{l}\text { Kress WJ et al., 1998. Amazonian biodiversity: } \\
\text { assessing conservation priorities with taxonomic data. } \\
\text { Biodiversity and Conservation, } 7: 1577-1587 \text {. }\end{array}$ \\
\hline 2002 & & & & & $\checkmark$ & & ND & & Land use & & $\begin{array}{l}\text { Veríssimo A et al., 2002. Priority Areas for Establishing } \\
\text { National Forests in the Brazilian Amazon. Conservation } \\
\text { Ecology, } 6 \text { (1):4. }\end{array}$ \\
\hline 2002 & $\checkmark$ & & & $\begin{array}{l}\text { Overall richness, } \\
\text { taxonomic }\end{array}$ & & & ND & & & & $\begin{array}{l}\text { Grelle CEV, 2002. Is higher-taxon analysis an useful } \\
\text { surrogate of species richness in studies of Neotropical } \\
\text { mammal diversity? Biological Conservation, 108:101- } \\
106 .\end{array}$ \\
\hline 2003 & & & & & $\checkmark$ & & ND & Default threshold & & & $\begin{array}{l}\text { Ferraz SF de B \& Vettorazzi CA, 2003. Identificação de } \\
\text { áreas para recomposição florestal com base em } \\
\text { princípios de ecologia de paisagem. Revista Árvore, } 27 \\
\text { (4): 575-583. }\end{array}$ \\
\hline 2003 & $\checkmark$ & & & Threatened & & & ND & & & & $\begin{array}{l}\text { Loiselle BA et al., 2003.Avoiding pitfalls of using } \\
\text { species-distribution models in conservation planning. } \\
\text { Conservation Biology, } 17 \text { (6):1-10. }\end{array}$ \\
\hline 2003 & & & & & $\checkmark$ & $\checkmark$ & ND & & & & $\begin{array}{l}\text { De Novaes P da C, Dias R \& Ferreira, LG, } 2003 . \\
\text { Identificação de áreas prioritárias para conservação da } \\
\text { biogeodiversidade no Estado de Goiás. Boletim Goiano } \\
\text { de Geografia, } 23 \text { (1):40-58. }\end{array}$ \\
\hline
\end{tabular}




\begin{tabular}{|c|c|c|c|c|c|c|c|c|c|c|c|}
\hline \multicolumn{8}{|c|}{ REPRESENTATIVENESS } & \multirow{2}{*}{\multicolumn{3}{|c|}{ PERSISTENCE }} & \multirow{4}{*}{ REFERENCES } \\
\hline \multicolumn{8}{|c|}{ FEATURES } & & & & \\
\hline \multicolumn{5}{|c|}{ SPECIES } & \multirow{2}{*}{ ENV. } & \multirow{2}{*}{ ENV. PROCESS } & \multirow{2}{*}{ FEATURES N ${ }^{0}$} & \multirow{2}{*}{ TARGETS } & \multirow{2}{*}{$\begin{array}{c}\text { STATIC } \\
\text { THREATS }\end{array}$} & \multirow{2}{*}{$\begin{array}{l}\text { DYNAMIC } \\
\text { THREATS }\end{array}$} & \\
\hline YEAR & $\mathbf{v}$ & I. & $\mathbf{P}$. & \begin{tabular}{|c} 
SPECIES DIVERSITY \\
INDEX
\end{tabular} & & & & & & & \\
\hline 2004 & $\checkmark$ & & & Endemism & & & 105 & Default threshold & $\begin{array}{l}\text { Land use/ socio- } \\
\text { economic } \\
\text { variables }\end{array}$ & & $\begin{array}{l}\text { Diniz-Filho JAF et al., 2004. Spatial patterns in species } \\
\text { richness and priority areas for conservation of anurans } \\
\text { in the Cerrado region, Central Brazil. Amphibia- } \\
\text { Reptilia, 25: 63-75. }\end{array}$ \\
\hline 2005 & $\checkmark$ & & & $\begin{array}{l}\text { Overall richness, } \\
\text { endemism }\end{array}$ & & & 105 & $\begin{array}{l}\text { Minimum viable } \\
\text { population }\end{array}$ & & & $\begin{array}{l}\text { Diniz-Filho JAF et al., 2005. Priority areas for anuran } \\
\text { conservation using biogeographical data: a comparison } \\
\text { of greedy, rarity, and simulated annealing algorithms to } \\
\text { define reserve networks in Cerrado. Brazilian Journal of } \\
\text { Biology, } 65 \text { (2):251-261. }\end{array}$ \\
\hline 2005 & $\checkmark$ & & & $\begin{array}{l}\text { Overall richness, } \\
\text { threatened, rarity, } \\
\text { flagship or/and } \\
\text { umbrella }\end{array}$ & & & 315 & & & & $\begin{array}{l}\text { Tognelli MF, 2005. Assessing the utility of indicator } \\
\text { groups for the conservation of South American } \\
\text { terrestrial mammals. Biological Conservation, 121(3): } \\
\text { 409-417. }\end{array}$ \\
\hline 2005 & & & & & $\checkmark$ & & 11 & Ecological threshold & & & $\begin{array}{l}\text { Anacleto TC da S et al., 2005. Seleção de áreas de } \\
\text { interesse ecológico através de sensoriamento remoto e } \\
\text { de otimização matemática: um estudo de caso no } \\
\text { município de Cocalinho, MT. Acta Amazonica, } 35 \text { (4), } \\
\text { 437-444. }\end{array}$ \\
\hline 2005 & & & & & $\checkmark$ & $\checkmark$ & ND & Landscape metrics & & & $\begin{array}{l}\text { Medeiros J de D, Savi M \& Brito BFAD, 2005. Seleção } \\
\text { de áreas para criação de Unidades de Conservação na } \\
\text { Floresta Ombrófila Mista. Biotemas, } 18 \text { (2), 33-50. }\end{array}$ \\
\hline 2006 & $\checkmark$ & & & Overall richness & & & 131 & Default threshold & $\begin{array}{l}\text { Land use and/or } \\
\text { socio-economic } \\
\text { variables }\end{array}$ & & $\begin{array}{l}\text { Silva RDJ et al., 2006. Malha rodoviária e conflitos de } \\
\text { conservação no cerrado: um estudo para a preservação } \\
\text { de anfíbios. Acta Scientiarum Biological Sciences, } 28 \\
\text { (4): 373-378. }\end{array}$ \\
\hline 2006 & $\checkmark$ & & & Overall richness & & & 131 & Default threshold & $\begin{array}{l}\text { Land use and/or } \\
\text { socio-economic } \\
\text { variables }\end{array}$ & & $\begin{array}{l}\text { Diniz-Filho JAF et al., 2007. Conservation } \\
\text { biogeography of anurans in Brazilian Cerrado. } \\
\text { Biodiversity and Conservation, 16:997-1008. }\end{array}$ \\
\hline 2006 & $\checkmark$ & & & Overall richness & & & 131 & Default threshold & $\begin{array}{l}\text { Land use and/or } \\
\text { socio-economic } \\
\text { variables }\end{array}$ & & $\begin{array}{l}\text { Diniz-Filho JAF et al., 2006. Anuran species richness, } \\
\text { complementarity and conservation conflicts in Brazilian } \\
\text { Cerrado. Acta Oecologica, 29: 9-15. }\end{array}$ \\
\hline 2006 & & & $\checkmark$ & Restricted-range & $\checkmark$ & $\checkmark$ & ND & & $\begin{array}{l}\text { Land use and/or } \\
\text { socio-economic } \\
\text { variables, } \\
\text { landscape } \\
\text { metrics, invasive } \\
\text { species }\end{array}$ & & $\begin{array}{l}\text { Durigan } \mathrm{G} \text { et al., 2006. Seleção de fragmentos } \\
\text { prioritários para a criação de unidades de conservação } \\
\text { do Cerrado no Estado de São Paulo. Revista do Instituto } \\
\text { Florestal, 18: 23-37. }\end{array}$ \\
\hline
\end{tabular}




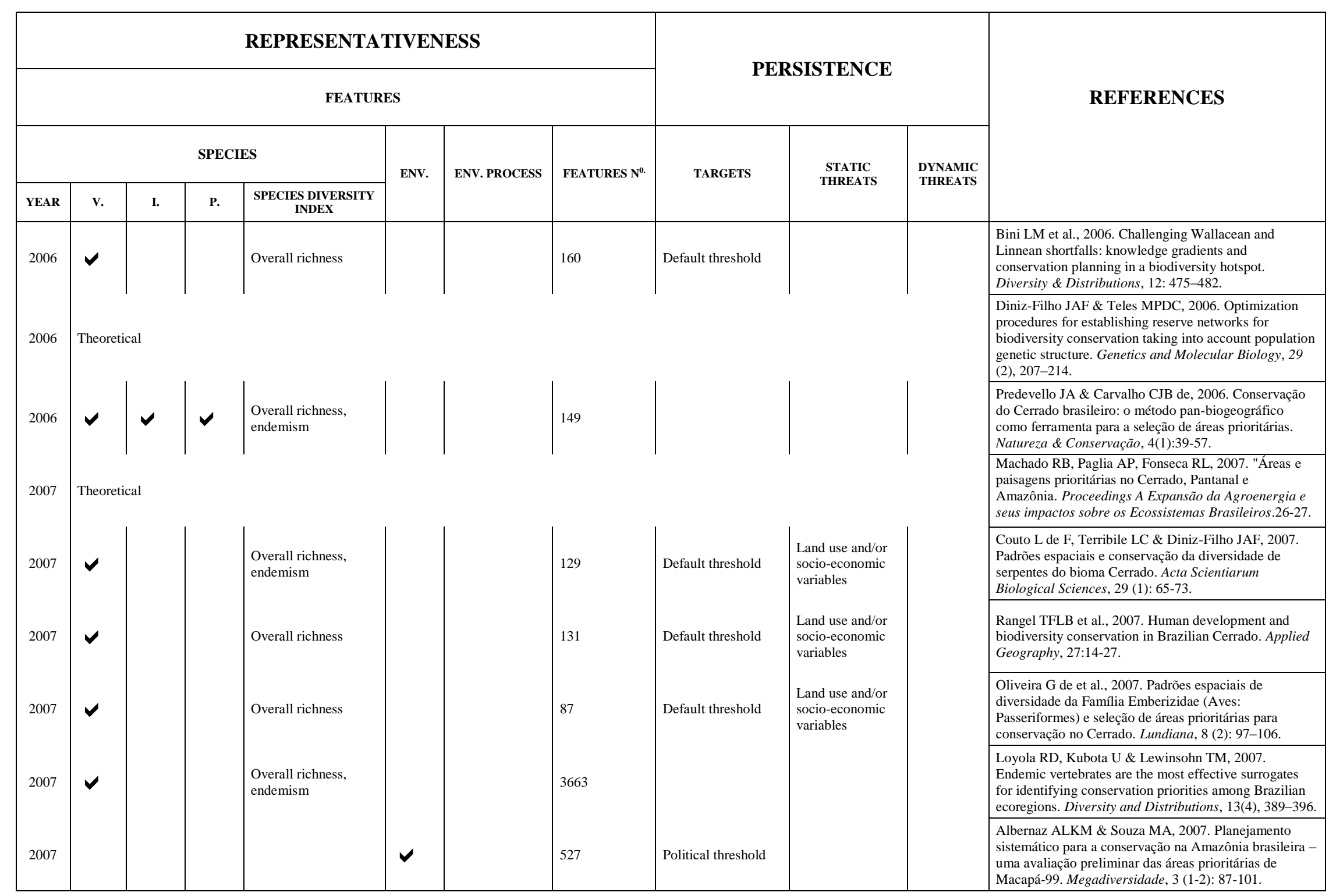




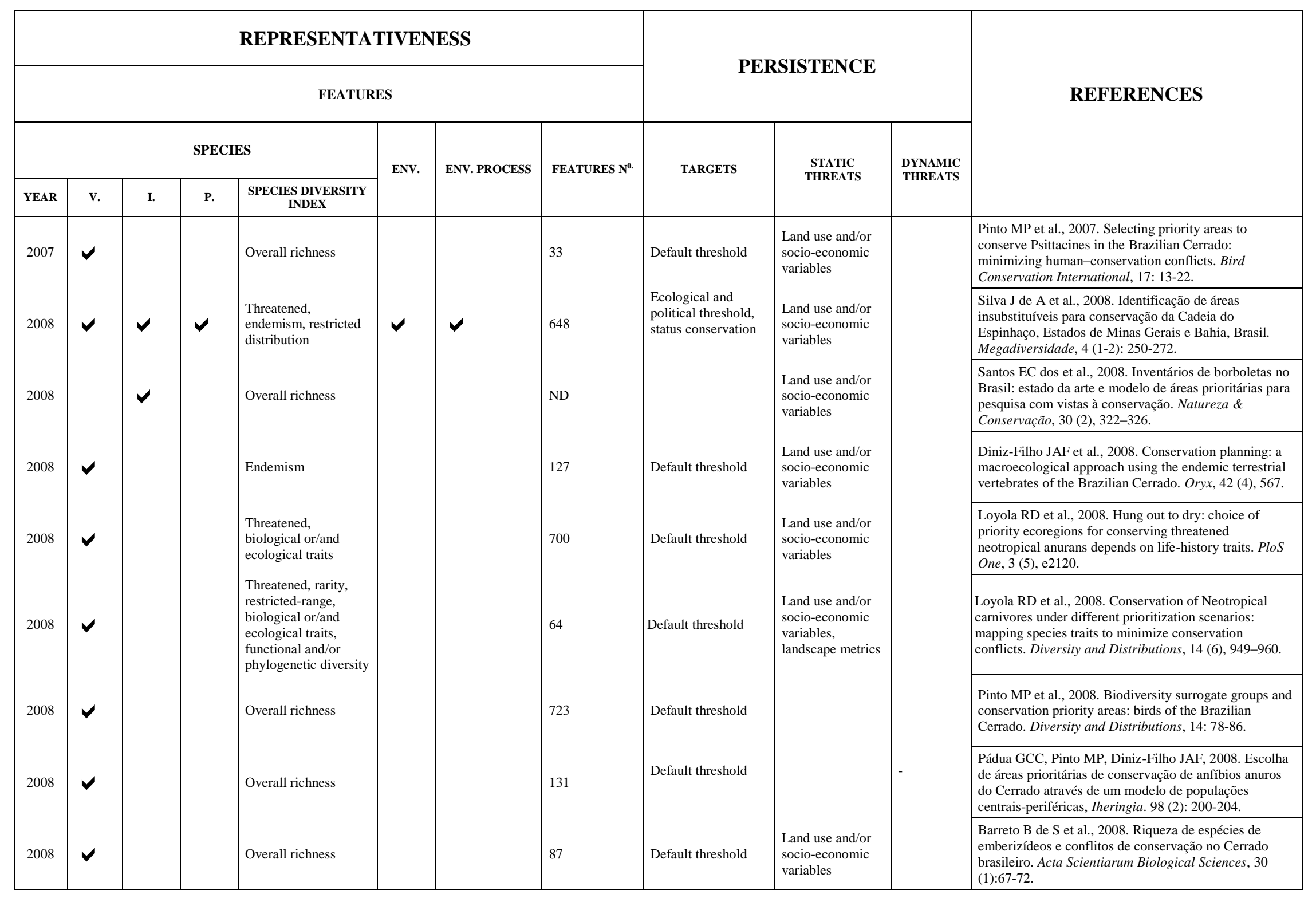




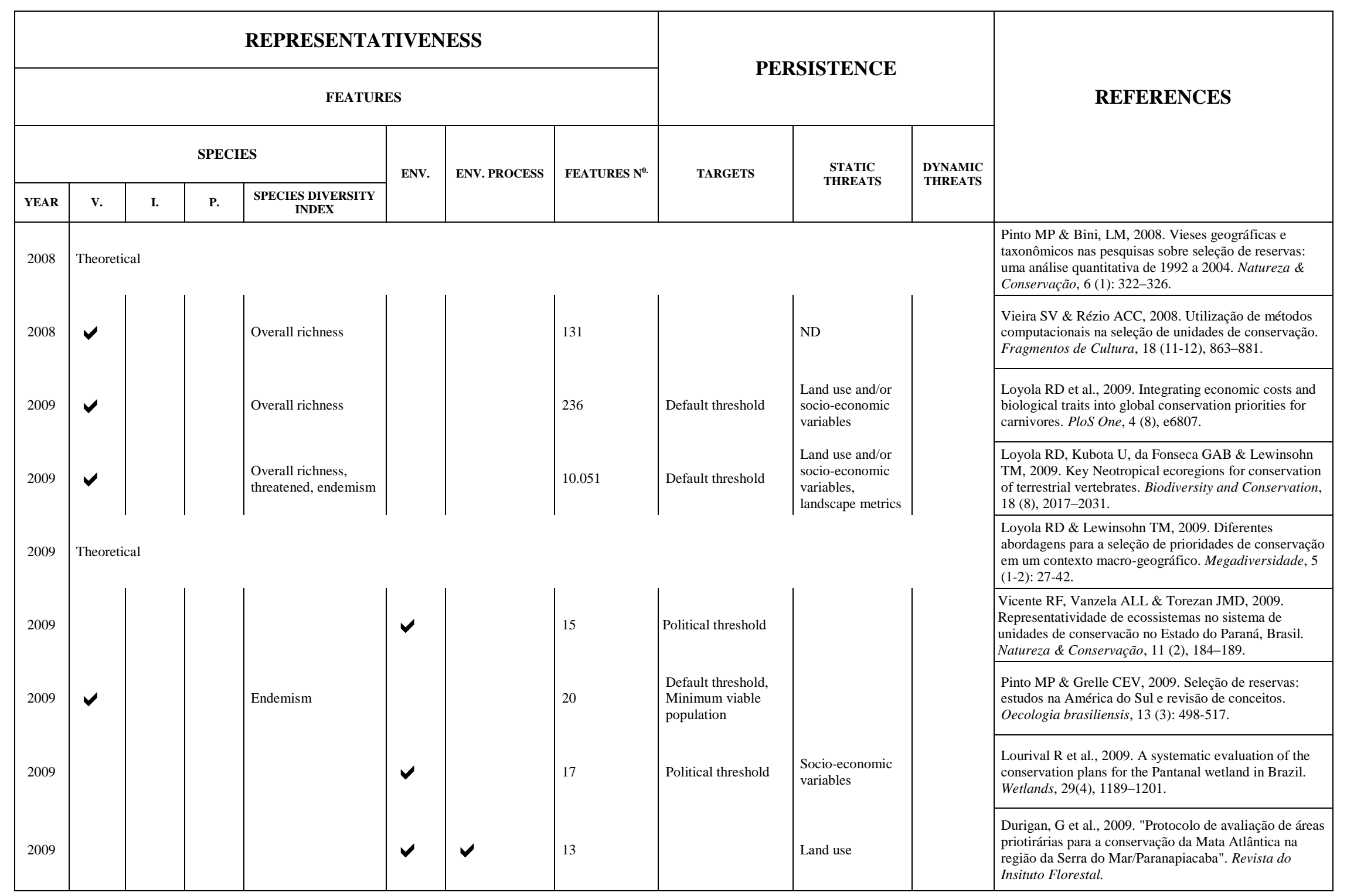




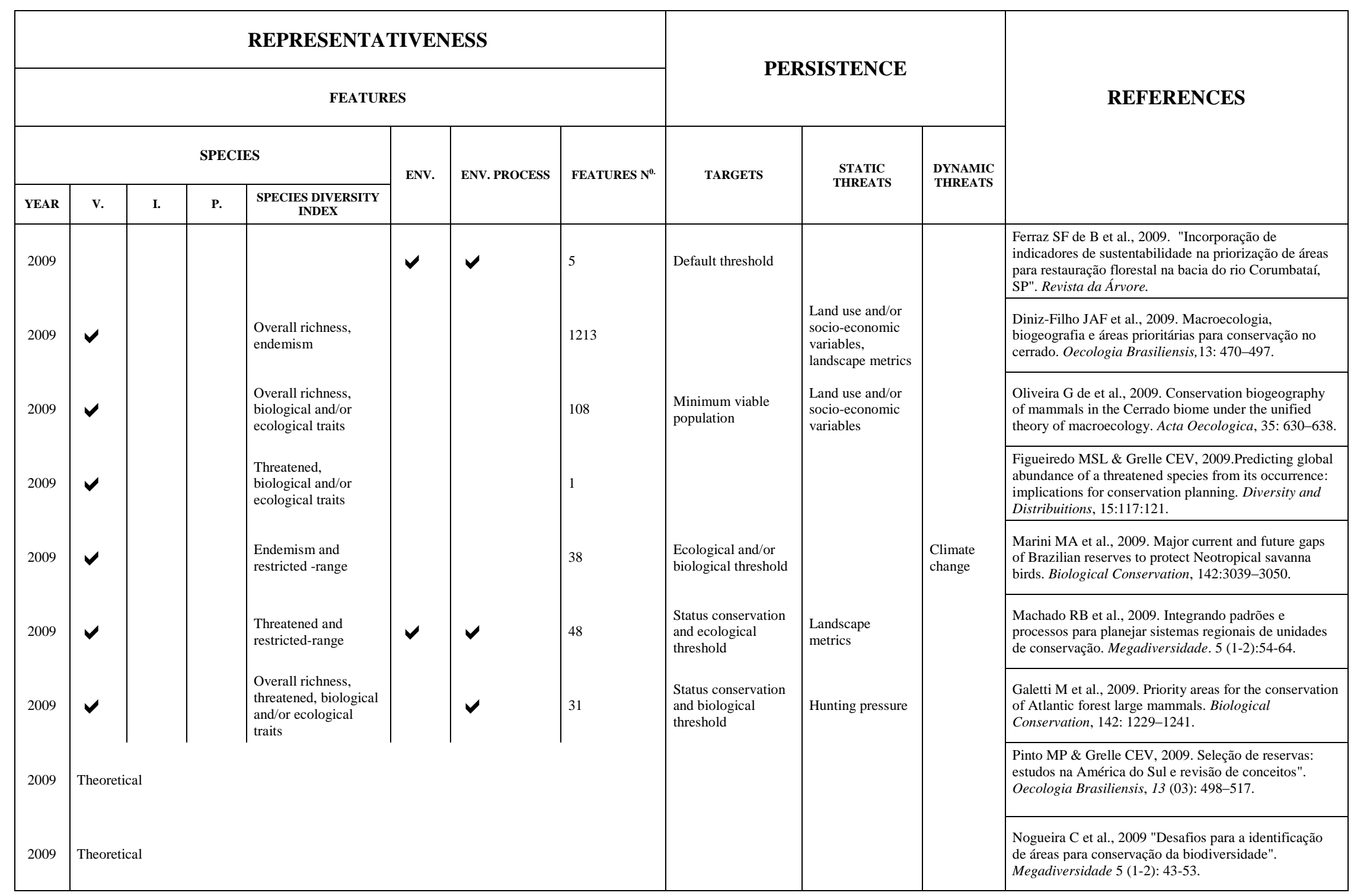




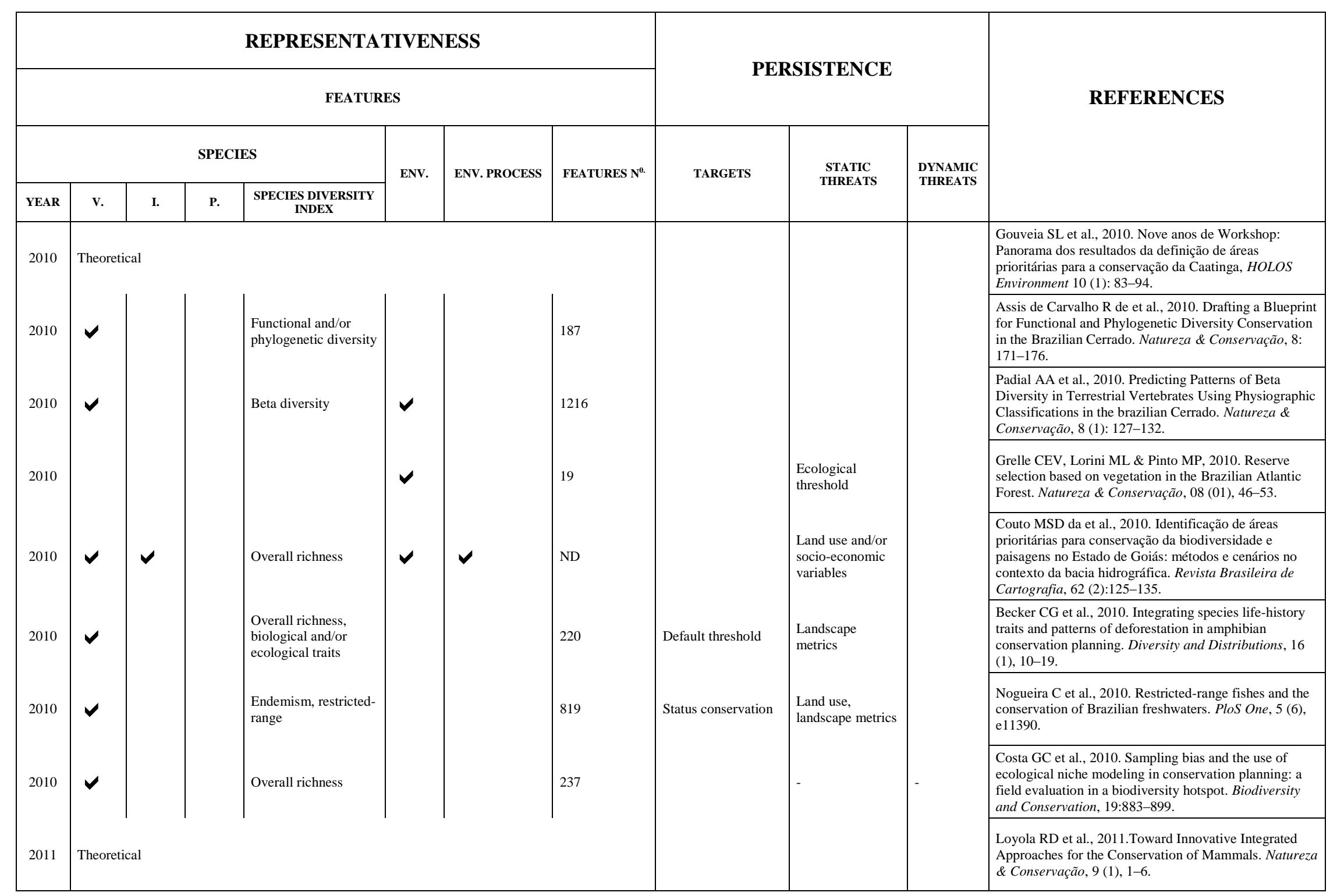




\begin{tabular}{|c|c|c|c|c|c|c|c|c|c|c|c|}
\hline \multicolumn{8}{|c|}{ REPRESENTATIVENESS } & \multirow{2}{*}{\multicolumn{3}{|c|}{ PERSISTENCE }} & \multirow{4}{*}{ REFERENCES } \\
\hline \multicolumn{8}{|c|}{ FEATURES } & & & & \\
\hline \multicolumn{5}{|c|}{ SPECIES } & \multirow{2}{*}{ ENV. } & \multirow{2}{*}{ ENV. PROCESS } & \multirow{2}{*}{ FEATURES $\mathbf{N}^{0}$} & \multirow{2}{*}{ TARGETS } & \multirow{2}{*}{$\begin{array}{l}\text { STATIC } \\
\text { THREATS }\end{array}$} & \multirow{2}{*}{$\begin{array}{l}\text { DYNAMIC } \\
\text { THREATS }\end{array}$} & \\
\hline YEAR & v. & I. & $\mathbf{P}$. & $\begin{array}{c}\text { SPECIES DIVERSITY } \\
\text { INDEX }\end{array}$ & & & & & & & \\
\hline 2011 & & $\checkmark$ & $\checkmark$ & Overall richness & & & 176 & & - & - & $\begin{array}{l}\text { Araújo WS de, 2011. Can host plant richness be used as } \\
\text { a surrogate for galling insect diversity? Tropical } \\
\text { Conservation Science, } 4 \text { (4), 420-427. }\end{array}$ \\
\hline 2011 & & & $\checkmark$ & $\begin{array}{l}\text { Alfa and Beta } \\
\text { diversity }\end{array}$ & & & 6 & & & & $\begin{array}{l}\text { Carvalho FA \& Felfili JM, 2011. Aplicação da } \\
\text { diversidade alfa e beta para definição de áreas } \\
\text { prioritárias para conservação: uma análise das florestas } \\
\text { deciduais sobre afloramentos calcários no Vale do } \\
\text { Paranã, Goiás. Bioscience Journal, } 27 \text { (5): 830-838. } \\
\end{array}$ \\
\hline 2011 & & & & & $\checkmark$ & $\checkmark$ & ND & & $\begin{array}{l}\text { Land use and/or } \\
\text { socio-economic } \\
\text { variables }\end{array}$ & & $\begin{array}{l}\text { Couto MS et al., 2011. Modelagem matemática para } \\
\text { seleção de áreas prioritárias à conservação ou } \\
\text { restauração no Cerrado goiano. Revista Mercator, } 10 \\
\text { (23): 225-a. }\end{array}$ \\
\hline 2011 & $\checkmark$ & & & Endemism & & & 19 & Default threshold & $\begin{array}{l}\text { Socio-economic } \\
\text { variables }\end{array}$ & & $\begin{array}{l}\text { Pinto MP \& Grelle CEV, 2011. Minimizing } \\
\text { conservation conflict for endemic primate species in } \\
\text { Atlantic forest and uncovering knowledge bias. } \\
\text { Environmental Conservation, } 39 \text { (1): } 30-37 . \\
\end{array}$ \\
\hline 2011 & & & & & $\checkmark$ & & 5 & Default threshold & 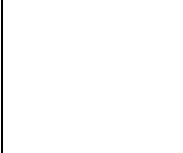 & LUCC & $\begin{array}{l}\text { Lourival R et al., 2011.Planning for reserve adequacy in } \\
\text { dynamic landscapes; maximizing future representation } \\
\text { of vegetation communities under flood disturbance in } \\
\text { the Pantanal wetland. Diversity and Distributions, } 17 \\
\text { (2), 297-310. }\end{array}$ \\
\hline 2011 & $\checkmark$ & & & $\begin{array}{l}\text { Endemism, } \\
\text { threatened, taxonomic } \\
\text { richness, restricted - } \\
\text { range }\end{array}$ & & & 392 & Default threshold & & & $\begin{array}{l}\text { Trindade-Filho J \& Loyola RD, 2011. Performance and } \\
\text { consistency of indicator groups in two biodiversity } \\
\text { hotspots. PloS One, } 6 \text { (5), e19746. }\end{array}$ \\
\hline 2011 & $\checkmark$ & & & & & $\checkmark$ & 308 & Status conservation & $\begin{array}{l}\text { Landscape } \\
\text { metrics }\end{array}$ & & $\begin{array}{l}\text { Albuquerque FS de et al., 2011. Identification of Critical } \\
\text { Areas for Mammal Conservation in the Brazilian } \\
\text { Atlantic Forest Biosphere Reserve. Natureza \& } \\
\text { Conservação, } 9 \text { (1):73-78. } \\
\end{array}$ \\
\hline 2011 & Theo & & & & & & & & & & $\begin{array}{l}\text { Lemes P et al., 2011. Refinando Dados Espaciais para a } \\
\text { Conservação da Biodiversidade. Natureza \& } \\
\text { Conservąão, } 9 \text { (2), 240-243. }\end{array}$ \\
\hline 2011 & $\checkmark$ & & & $\begin{array}{l}\text { Temporal variability, } \\
\text { biological and/or } \\
\text { ecological traits }\end{array}$ & & & 39 & Default threshold & $\begin{array}{l}\text { Landscape } \\
\text { metrics }\end{array}$ & & $\begin{array}{l}\text { Felinks B et al., 2011. Effects of species turnover on } \\
\text { reserve site selection in a fragmented landscape. } \\
\text { Biodiversity and Conservation, } 20 \text { (5), 1057-1072. }\end{array}$ \\
\hline
\end{tabular}




\begin{tabular}{|c|c|c|c|c|c|c|c|c|c|c|c|}
\hline \multicolumn{8}{|c|}{ REPRESENTATIVENESS } & \multirow{2}{*}{\multicolumn{3}{|c|}{ PERSISTENCE }} & \multirow{4}{*}{ REFERENCES } \\
\hline \multicolumn{8}{|c|}{ FEATURES } & & & & \\
\hline \multicolumn{5}{|c|}{ SPECIES } & \multirow{2}{*}{ ENV. } & \multirow{2}{*}{ ENV. PROCESS } & \multirow{2}{*}{ FEATURES $\mathbf{N}^{0}$} & \multirow{2}{*}{ TARGETS } & \multirow{2}{*}{$\begin{array}{l}\text { STATIC } \\
\text { THREATS }\end{array}$} & \multirow{2}{*}{$\begin{array}{l}\text { DYNAMIC } \\
\text { THREATS }\end{array}$} & \\
\hline YEAR & v. & I. & P. & $\begin{array}{l}\text { SPECIES DIVERSITY } \\
\text { INDEX }\end{array}$ & & & & & & & \\
\hline 2012 & $\checkmark$ & & & Threatened/DD & & & 104 & $\begin{array}{l}\text { Ecological and/or } \\
\text { biological threshold }\end{array}$ & & & $\begin{array}{l}\text { Trindade-Filho J et al., 2012. How does the inclusion of } \\
\text { Data Deficient species change conservation priorities for } \\
\text { amphibians in the Atlantic. Biodiversity and } \\
\text { Conservation, 21:2709-2718. }\end{array}$ \\
\hline 2012 & $\checkmark$ & & & $\begin{array}{l}\text { Functional and/or } \\
\text { phylogenetic diversity }\end{array}$ & & & 854 & Default threshold & & & $\begin{array}{l}\text { Trindade-Filho J et al., 2012. Using indicator groups to } \\
\text { represent bird phylogenetic and functional diversity. } \\
\text { Biological Conservation, 146: } 155-162 \text {. }\end{array}$ \\
\hline 2012 & & & $\checkmark$ & & & & & ND & $\begin{array}{l}\text { Land use and/or } \\
\text { socio-economic } \\
\text { variables }\end{array}$ & & $\begin{array}{l}\text { Albernaz AL et al., 2012. Tree species compositional } \\
\text { change and conservation implications in the white-water } \\
\text { flooded forests of the Brazilian Amazon. Journal of } \\
\text { Biogeography, 39: 869-883. }\end{array}$ \\
\hline 2012 & & & $\checkmark$ & Genetic & & & 25 & & $\begin{array}{l}\text { Landscape } \\
\text { metrics }\end{array}$ & & $\begin{array}{l}\text { Diniz-Filho JAF et al., 2012. Planning for optimal } \\
\text { conservation of geographical genetic variability within } \\
\text { species. Conservation Genetics, 13:1085-1093. }\end{array}$ \\
\hline 2012 & $\checkmark$ & $\checkmark$ & $\checkmark$ & $\begin{array}{l}\text { Overall richness, } \\
\text { taxonomic }\end{array}$ & & & 22 & & 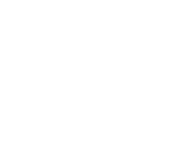 & & $\begin{array}{l}\text { Landeiro VL et al., 2012. How far can we go in } \\
\text { simplifying biomonitoring assessments? An integrated } \\
\text { analysis of taxonomic surrogacy, taxonomic sufficiency } \\
\text { and numerical resolution in a megadiverse region. } \\
\text { Ecological Indicators, } 23: 366-373 \text {. }\end{array}$ \\
\hline 2012 & $\checkmark$ & & & $\begin{array}{l}\text { Overall richness, } \\
\text { threatened }\end{array}$ & & & 814 & Political threshold & & LUCC & $\begin{array}{l}\text { Bird JP et al., 2012. Integrating spatially explicit habitat } \\
\text { projections into extinction risk assessments: a } \\
\text { reassessment of Amazonian avifauna incorporating } \\
\text { projected deforestation. Diversity and Distributions, } \\
\text { 18:273-281. }\end{array}$ \\
\hline 2013 & & & & & $\checkmark$ & & 8 & $\begin{array}{l}\text { biological and/or } \\
\text { ecological threshold }\end{array}$ & $\begin{array}{l}\text { Landscape } \\
\text { metrics }\end{array}$ & & $\begin{array}{l}\text { Magris RA et al., 2013. Analysis of Progress Towards a } \\
\text { Comprehensive System of Marine Protected Areas in } \\
\text { Brazil. Nat. Conserv. 11:1-7. }\end{array}$ \\
\hline 2013 & $\checkmark$ & & & $\begin{array}{l}\text { Threatened, flagship } \\
\text { and/ or umbrella, } \\
\text { functional and/or } \\
\text { phylogenetic diversity }\end{array}$ & & & 701 & $\begin{array}{l}\text { Status conservation, } \\
\text { biological and/or } \\
\text { ecological threshold }\end{array}$ & & & $\begin{array}{l}\text { Alves DMCC. \& Brito, D., 2013. Priority mammals for } \\
\text { biodiversity conservation in Brazil. Trop. Conserv. Sci. } \\
\text { 6: 558-583. }\end{array}$ \\
\hline 2013 & $\checkmark$ & & & Threatened & & & 154 & Political threshold & & LUCC & $\begin{array}{l}\text { Faleiro FV, Machado RB, Loyola RD, 2013. Defining } \\
\text { spatial conservation priorities in the face of land-use } \\
\text { and climate change. Biol. Conserv. 158: } 248-257\end{array}$ \\
\hline
\end{tabular}




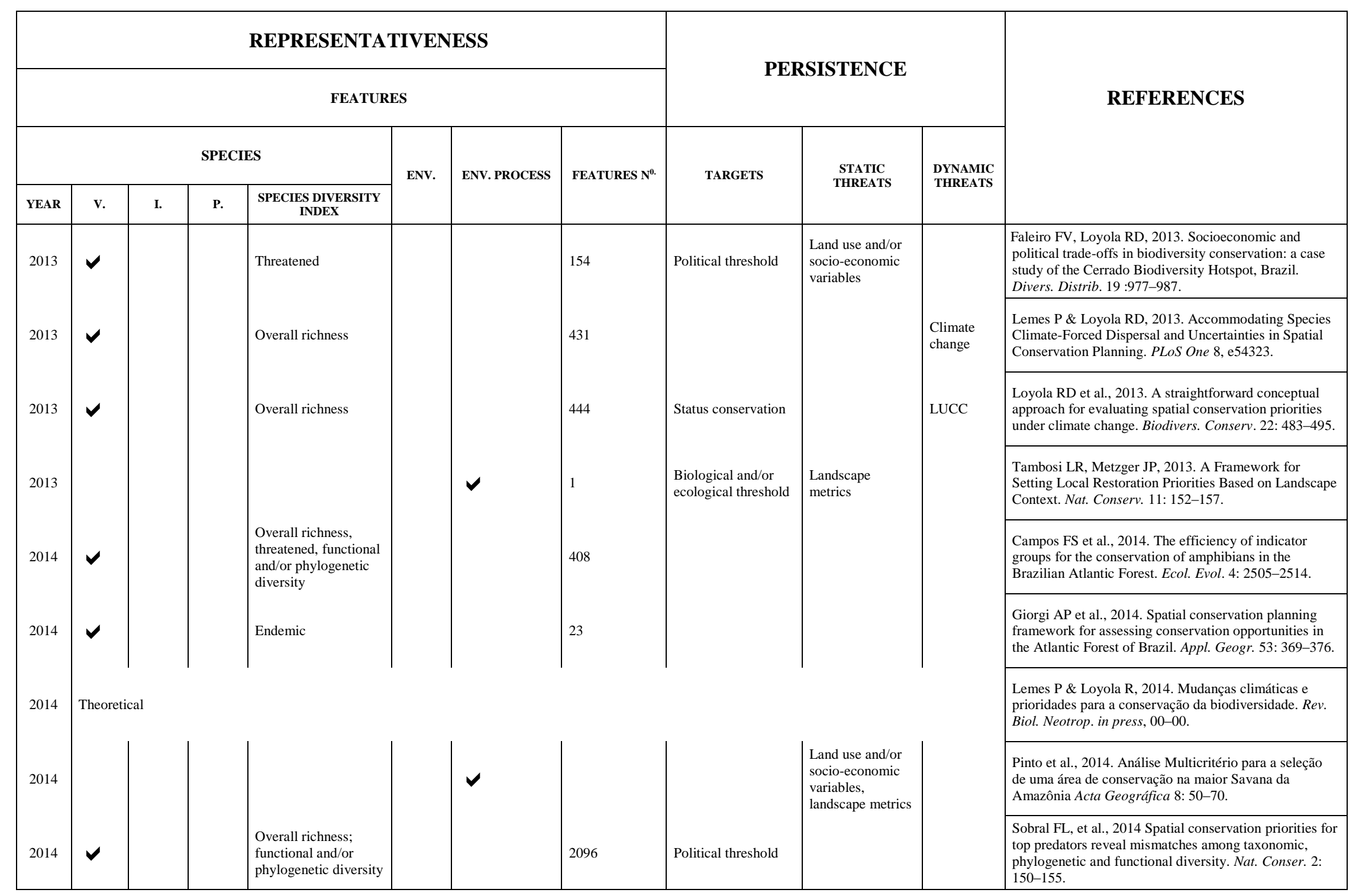




\begin{tabular}{|c|c|c|c|c|c|c|c|c|c|c|c|}
\hline \multicolumn{8}{|c|}{ REPRESENTATIVENESS } & \multirow{2}{*}{\multicolumn{3}{|c|}{ PERSISTENCE }} & \multirow{4}{*}{ REFERENCES } \\
\hline \multicolumn{8}{|c|}{ FEATURES } & & & & \\
\hline \multicolumn{5}{|c|}{ SPECIES } & \multirow{2}{*}{ ENV. } & \multirow{2}{*}{ ENV. PROCESS } & \multirow{2}{*}{ FEATURES $\mathbf{N}^{0}$} & \multirow{2}{*}{ TARGETS } & \multirow{2}{*}{$\begin{array}{c}\text { STATIC } \\
\text { THREATS }\end{array}$} & \multirow{2}{*}{$\begin{array}{l}\text { DYNAMIC } \\
\text { THREATS }\end{array}$} & \\
\hline YEAR & v. & I. & P. & $\begin{array}{l}\text { INDEX } \\
\text { INDE }\end{array}$ & & & & & & & \\
\hline 2014 & $\checkmark$ & & & Endemic & & & 30 & $\begin{array}{l}\text { Status conservation, } \\
\text { biological and/or } \\
\text { ecological }\end{array}$ & & LUCC & $\begin{array}{l}\text { Silva VDNE et al., 2014. Formulating conservation } \\
\text { targets for a gap analysis of endemic lizards in a } \\
\text { biodiversity hotspot. Biol. Conserv. 180, } 1-10 \text {. }\end{array}$ \\
\hline 2015 & & & $\checkmark$ & $\begin{array}{l}\text { Overall richness, } \\
\text { functional and/or } \\
\text { phylogenetic } \\
\text { diversity, restricted - } \\
\text { range, endemic }\end{array}$ & & & 25 & $\begin{array}{l}\text { Status conservation, } \\
\text { biological and/or } \\
\text { ecological threshold }\end{array}$ & & & $\begin{array}{l}\text { Menezes MOT \& Ribeiro-Silva S, 2015. Cactáceas do } \\
\text { Ceará, Brasil: Prioridades para a conservação. Gaia Sci. } \\
\text { 9: 67-76. }\end{array}$ \\
\hline 2015 & $\checkmark$ & & & $\begin{array}{l}\text { Biological and/or } \\
\text { ecological traits }\end{array}$ & & & 2 & Political threshold & $\begin{array}{l}\text { Land use and/or } \\
\text { socio-economic } \\
\text { variables, } \\
\text { landscape metrics }\end{array}$ & & $\begin{array}{l}\text { Crouzeilles R et al., 2015. Incorporating habitat } \\
\text { availability into systematic planning for restoration: a } \\
\text { species-specific approach for Atlantic Forest mammals. } \\
\text { Divers. Distrib. 1-11. }\end{array}$ \\
\hline 2015 & $\checkmark$ & & & Overall richness & & & 47 & $\begin{array}{l}\text { Status conservation, } \\
\text { biological and/or } \\
\text { ecological threshold, }\end{array}$ & $\begin{array}{l}\text { Land use and/or } \\
\text { socio-economic } \\
\text { variables }\end{array}$ & & $\begin{array}{l}\text { Gaiarsa MP et al., 2015. Setting conservation priorities } \\
\text { within monophyletic groups: An integrative approach. } J \text {. } \\
\text { Nat. Conserv. 24: 49-55. }\end{array}$ \\
\hline 2015 & $\checkmark$ & & & Overall richness & & & 745 & $\begin{array}{l}\text { Status conservation, } \\
\text { political threshold }\end{array}$ & $\begin{array}{l}\text { Land use and/or } \\
\text { socio-economic } \\
\text { variables }\end{array}$ & & $\begin{array}{l}\text { Vilar CC, Joyeux JC, Loyola R., Spach HL, } 2015 . \\
\text { Setting priorities for the conservation of marine } \\
\text { vertebrates in Brazilian waters. Ocean Coast. Manag. } \\
\text { 107: } 28-36 .\end{array}$ \\
\hline
\end{tabular}

Shaffer SL, 1981. Minimum population sizes for species conservation. BioScience. 31:131-134 
Table S5: The representativeness and persistence in the practice. Features: types of species (V=vertebrates; I=invertebrates; P=plants), species diversity indexes, environmental (e.g. vegetation), environmental process (e.g. fragmentation, refuges). Targets: default threshold (uniform goals), political threshold (legally established goals), biological and ecological threshold (goals according to biological and ecological traits of features), minimum viable population size (Shaffer, 1981), conservation status and others. Threats: static (considering the current conditions) or dynamic (predictive models).

\begin{tabular}{|c|c|c|c|c|c|c|c|c|c|c|c|}
\hline \multicolumn{8}{|c|}{ REPRESENTATIVENESS } & \multirow{2}{*}{\multicolumn{3}{|c|}{ PERSISTENCE }} & \multirow{4}{*}{ REFERENCES } \\
\hline \multicolumn{8}{|c|}{ FEATURES } & & & & \\
\hline \multicolumn{5}{|c|}{ SPECIES } & \multirow{2}{*}{ ENV. } & \multirow{2}{*}{$\begin{array}{l}\text { ENV. } \\
\text { PROCESS }\end{array}$} & \multirow{2}{*}{$\underset{\mathbf{N}^{0 .}}{\text { FEATUS }}$} & \multirow{2}{*}{ TARGETS } & \multirow{2}{*}{$\begin{array}{l}\text { STATIC } \\
\text { THREATS }\end{array}$} & \multirow{2}{*}{$\begin{array}{l}\text { DYNAMIC } \\
\text { THREATS }\end{array}$} & \\
\hline YEAR & v. & I. & $\mathbf{P}$ & $\begin{array}{l}\text { SPECIES DIVERSITY } \\
\text { INDEX }\end{array}$ & & & & & & & \\
\hline $\begin{array}{l}1976 / \\
1990\end{array}$ & $\checkmark$ & $\checkmark$ & $v$ & Overall richness, endemism & $\checkmark$ & $\checkmark$ & $\mathrm{ND}^{3}$ & & Land use & & $\begin{array}{l}\text { Wetterberg, GB et al., 1976. Uma Análise de Prioridades da } \\
\text { Conservação da Natureza na Amazônia. } \\
\text { PRODEPEF/UNDP/FAO/IBDF/BRA-45. Série Técnica } 8 . \\
\text { Rylands AB, 1990. Priority areas for conservation in the Amazon. } \\
\text { Trends in Ecology \& Evolution. } 5 \text { (8):240-214. }\end{array}$ \\
\hline 1993 & $?^{4}$ & ? & $?$ & Overall richness, endemism & $\checkmark$ & & ND & & Landscape metrics & & $\begin{array}{l}\text { Conservação Internacional do Brasil (CI-Brasil) et al., 1994. Workshop } \\
\text { "Áreas prioritárias para conservação da Mata Atlântica Nordeste, } \\
\text { 1993". Pernambuco/ Belo Horizonte, CI-Brasil/ Fundação Biodiversitas } \\
\text { \& Sociedade Nordestina de Ecologia. }\end{array}$ \\
\hline 1998 & $\checkmark$ & $\checkmark$ & $\checkmark$ & $\begin{array}{l}\text { Overall richness, endemism, } \\
\text { rarity, threatened, restricted- } \\
\text { range. }\end{array}$ & $\checkmark$ & $\checkmark$ & ND & & $\begin{array}{l}\text { Land use, } \\
\text { landscape metrics }\end{array}$ & & $\begin{array}{l}\text { Drummond et al., 1998. Biodiversidade em Minas Gerais: Um Atlas } \\
\text { para sua Conservação. Belo Horizonte, Fundação Biodiversitas. }\end{array}$ \\
\hline 1998 & $\checkmark$ & $\checkmark$ & $\checkmark$ & $\begin{array}{l}\text { Overall richness, } \\
\text { Endemism, threatened, } \\
\text { rarity, migratory, others. }\end{array}$ & $\checkmark$ & & ND & & Land use & & $\begin{array}{l}\text { Brasil, 2002. Cerrado e Pantanal In: Biodiversidade brasileira: } \\
\text { Avaliação e identificação de áreas e ações prioritárias para } \\
\text { conservação, utilização sustentável e repartição dos benefícios da } \\
\text { biodiversidade nos biomas brasileiros. Brasília, MMA/SBF: 175-214. }\end{array}$ \\
\hline & & & & & & & & & & & $\begin{array}{l}\text { Brasil, 2007. "Biodiversidade do Cerrado e Pantanal: áreas e accoes } \\
\text { prioritárias para conservação da biodiversidade". Brasília, MMA: 540p. }\end{array}$ \\
\hline 1999 & $\checkmark$ & $\checkmark$ & $\checkmark$ & $\begin{array}{l}\text { Overall richness, } \\
\text { threatened, endemic, } \\
\text { restricted-range }\end{array}$ & $\checkmark$ & & ND & & $\begin{array}{l}\text { Natural dynamic, } \\
\text { land use. } \\
\text { overfishing }\end{array}$ & & $\begin{array}{l}\text { Brasil, 2002. Zona Costeira e Marinha In: Biodiversidade brasileira: } \\
\text { Avaliação e identificação de áreas e açães prioritárias para } \\
\text { conservaçãoo, utilizaçâo sustentável erepartição dos benefícios da } \\
\text { biodiversidade nos biomas brasileiros. Brasília, MMA/SBF: } 267-340 \text {. }\end{array}$ \\
\hline 1999 & $\checkmark$ & $\checkmark$ & $\checkmark$ & $\begin{array}{l}\text { Overall richness, endemism, } \\
\text { threatened, rarity }\end{array}$ & $\checkmark$ & $\checkmark$ & ND & & $\begin{array}{l}\text { Land use, } \\
\text { fragmentation }\end{array}$ & & $\begin{array}{l}\text { Conservação Internacional do Brasil et al., 2000. Avaliação e ações } \\
\text { prioritárias para a conservação da biodiversidade da Mata Atlântica e } \\
\text { Campos Sulinos. Brasília, MMA/SBF: } 40 \mathrm{p} \text {. }\end{array}$ \\
\hline
\end{tabular}

${ }^{3}$ Not described

${ }^{4}$ We couldn't access the fully information 


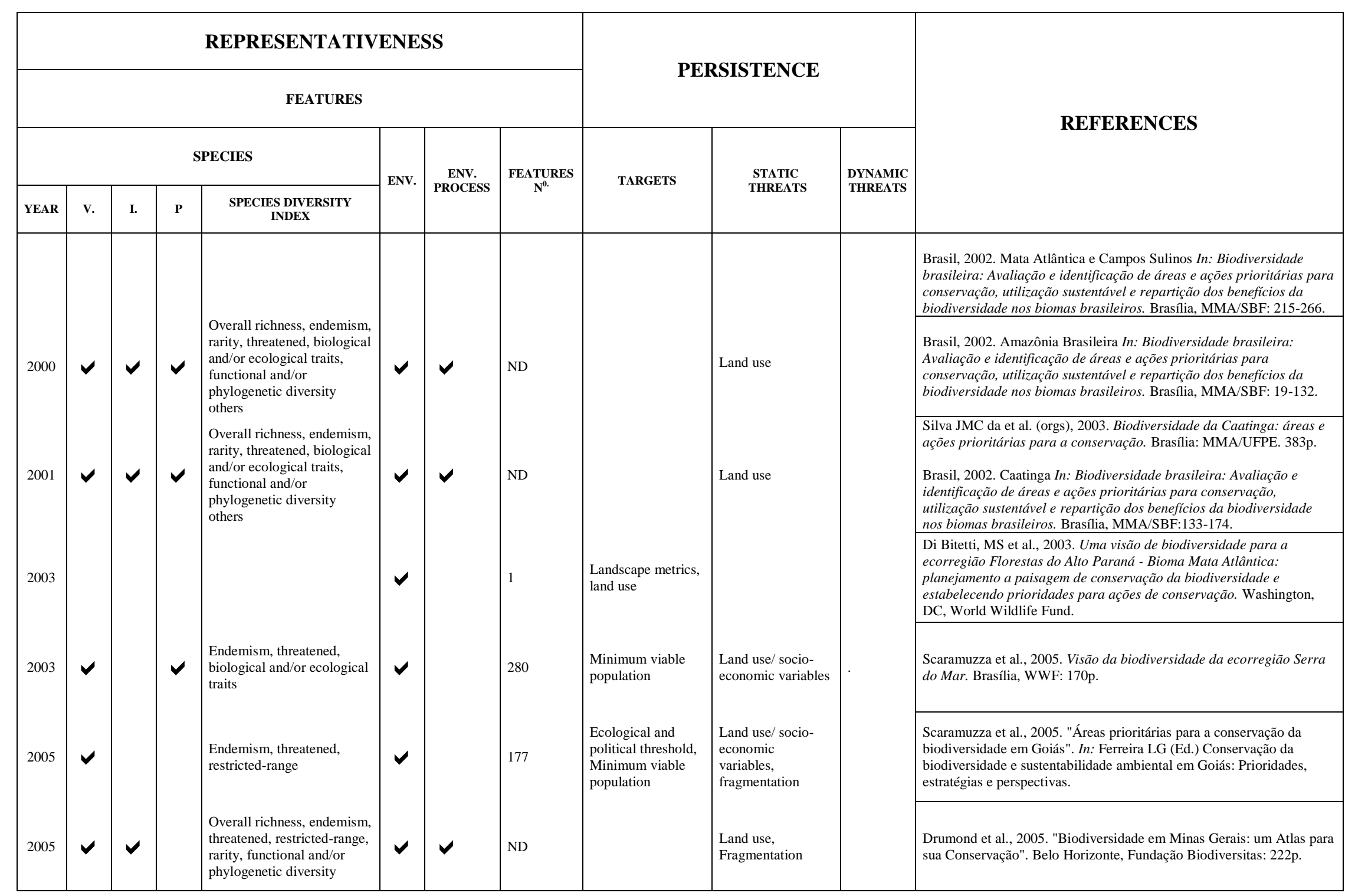




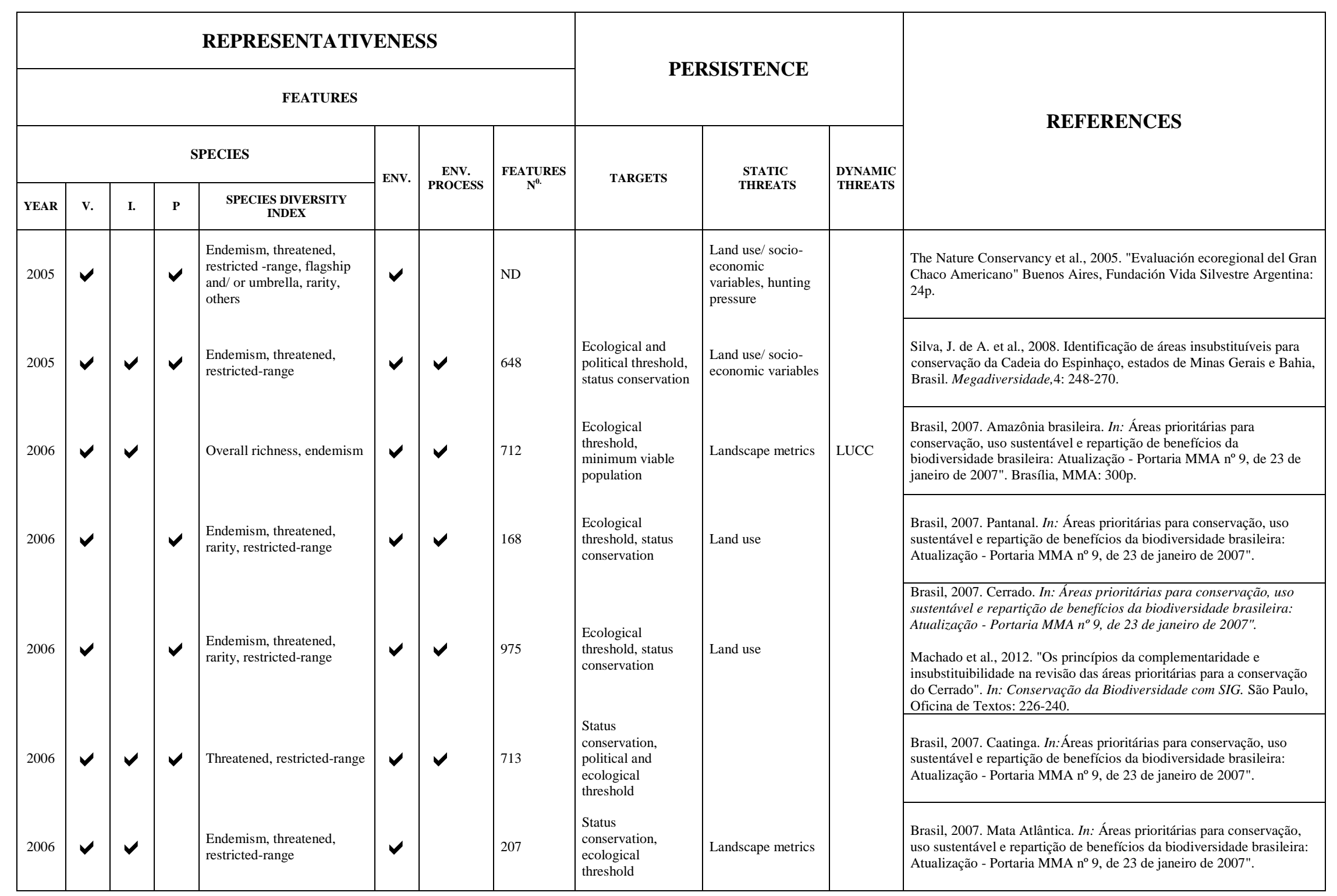




\begin{tabular}{|c|c|c|c|c|c|c|c|c|c|c|c|}
\hline \multicolumn{8}{|c|}{ REPRESENTATIVENESS } & \multirow{2}{*}{\multicolumn{3}{|c|}{ PERSISTENCE }} & \multirow{4}{*}{ REFERENCES } \\
\hline \multicolumn{8}{|c|}{ FEATURES } & & & & \\
\hline \multicolumn{5}{|c|}{ SPECIES } & \multirow{2}{*}{ ENV. } & \multirow{2}{*}{$\begin{array}{l}\text { ENV. } \\
\text { PROCESS }\end{array}$} & \multirow{2}{*}{$\begin{array}{l}\text { FEATURES } \\
\mathbf{N}^{0 .}\end{array}$} & \multirow{2}{*}{ TARGETS } & \multirow{2}{*}{$\begin{array}{l}\text { STATIC } \\
\text { THREATS }\end{array}$} & \multirow{2}{*}{$\begin{array}{l}\text { DYNAMIC } \\
\text { THREATS }\end{array}$} & \\
\hline YEAR & v. & I. & $\mathbf{P}$ & $\begin{array}{l}\text { SPECIES DIVERSITY } \\
\text { INDEX }\end{array}$ & & & & & & & \\
\hline 2006 & $\checkmark$ & $\checkmark$ & & - & $\checkmark$ & & 116 & $\begin{array}{l}\text { Status } \\
\text { conservation, } \\
\text { ecological } \\
\text { threshold }\end{array}$ & Land use & & $\begin{array}{l}\text { Brasil, 2007. Pampa. In: Áreas prioritárias para conservação, uso } \\
\text { sustentável e repartição de benefícios da biodiversidade brasileira: } \\
\text { Atualização - Portaria MMA n⿳ } 9 \text {, de } 23 \text { de janeiro de } 2007 " \text {. }\end{array}$ \\
\hline 2006 & $\checkmark$ & $\checkmark$ & & - & $\checkmark$ & & 239 & $\begin{array}{l}\text { Ecological } \\
\text { threshold and status } \\
\text { conservation }\end{array}$ & & & $\begin{array}{l}\text { Brasil, 2007. Zona Costeira e marinha. In:Áreas prioritárias para } \\
\text { conservação, uso sustentável e repartição de benefícios da } \\
\text { biodiversidade brasileira: Atualização - Portaria MMA nº } 9 \text {, de } 23 \text { de } \\
\text { janeiro de 2007". }\end{array}$ \\
\hline 2006 & $\checkmark$ & $\checkmark$ & $\checkmark$ & $\begin{array}{l}\text { Endemism, threatened, } \\
\text { rarity, biological and/or } \\
\text { ecological traits, restricted- } \\
\text { range }\end{array}$ & $\checkmark$ & & 10585 & Landscape metrics & Land use & & $\begin{array}{l}\text { Rodrigues et al. (orgs.), 2008. Diretrizes para a conservação e } \\
\text { restauração da biodiversidade no Estado de São Paulo. São Paulo, } \\
\text { SMA: 250p. }\end{array}$ \\
\hline 2006 & $\checkmark$ & $\checkmark$ & $\checkmark$ & $\begin{array}{l}\text { Endemism, threatened, } \\
\text { restricted-range }\end{array}$ & $\checkmark$ & $\checkmark$ & 345 & $\begin{array}{l}\text { Status } \\
\text { conservation, } \\
\text { ecological } \\
\text { threshold }\end{array}$ & $\begin{array}{l}\text { Land use/ socio- } \\
\text { economic variables }\end{array}$ & & $\begin{array}{l}\text { Brasil, 2011. Diagnóstico do macrozoneamento ecológico-econômico } \\
\text { da Bacia Hidrográfica do Rio Francisco. Brasília, MMA: 488p. }\end{array}$ \\
\hline 2006 & $?$ & $?$ & $?$ & $\begin{array}{l}\text { Overall richness, endemism, } \\
\text { threatened, restricted-range, } \\
\text { rarity, others }\end{array}$ & $\checkmark$ & & $?$ & & Landscape metrics & & $\begin{array}{l}\text { Araújo, M \& Souza, PVN, 2009. Plano de ação para a conservação da } \\
\text { biodiversidade do sul da Bahia. Ilhéus, IESB: 37p. }\end{array}$ \\
\hline 2011 & $?$ & $?$ & $?$ & $\begin{array}{l}\text { Endemism, threatened, } \\
\text { rarity, biological and/or } \\
\text { ecological traits }\end{array}$ & $\checkmark$ & $\checkmark$ & $?$ & & Land use & & $\begin{array}{l}\text { Ipema, 2011. Áreas e ações prioritárias para a conservação da } \\
\text { biodiversidade no estado do Espírito Santo. Vitória, Instituto de } \\
\text { Pesquisas da Mata Atlântica: 64p. }\end{array}$ \\
\hline 2012 & $\checkmark$ & $\checkmark$ & & $\begin{array}{l}\text { Endemism, threatened, } \\
\text { ecological and economical } \\
\text { traits }\end{array}$ & $\checkmark$ & - & ND & & $\begin{array}{l}\text { Land use/ socio- } \\
\text { economic variables }\end{array}$ & & $\begin{array}{l}\text { Brasil, 2012. Proposição de Unidades de Conservação na Região dos } \\
\text { Abrolhos. 27p. }\end{array}$ \\
\hline
\end{tabular}

Shaffer SL, 1981. Minimum population sizes for species conservation. BioScience. 31:131-134 


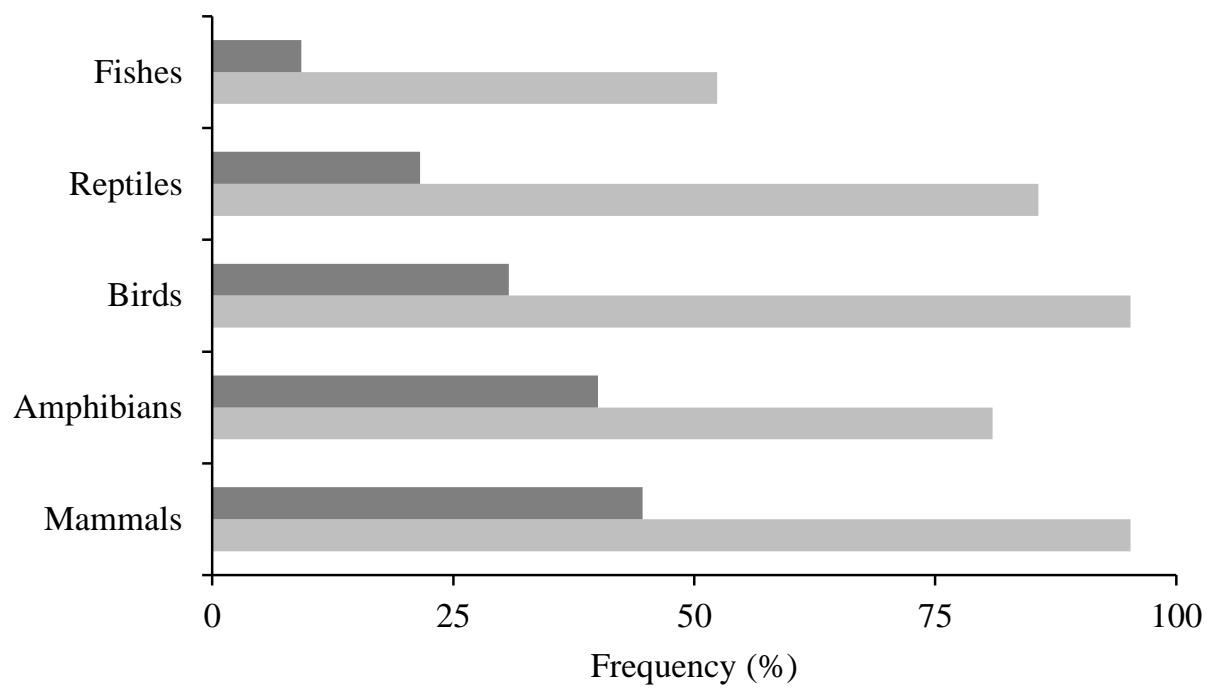

Figure S6: Frequency of features (groups of vertebrates) in science (dark grey) and in practice (light grey). 


\title{
CAPÍTULO 2
}

\section{CENÁRIOS PARA A CONSERVAÇÃO DA BIODIVERDADE NO BRASIL: AS METAS PARA A CONVENÇÃO SOBRE DIVERSIDADE BIOLÓGICA SÃO SUFICIENTES?}

\begin{abstract}
RESUMO
O Brasil como signatário da CDB acordou metas para a conservação das espécies e ecossistemas, e diminuição das perdas de hábitats. Considerada a principal causa da perda da biodiversidade. Modelos sobre a dinâmica do uso e ocupação do solo (LUCC) podem antecipar os riscos sobre o território. A partir desses modelos desenvolvemos cenários Business as usual - "BAU" (manutenção das atuais tendências de desflorestamento) e "GOV", construídos de acordo com algumas das metas estipuladas pelo Brasil para a CDB, por um período de 40 anos. Medimos a evolução das áreas remanescentes e métricas da paisagem nestes cenários; a vulnerabilidade dos ecossistemas e a representatividade ecossistêmica de classes de vegetação. Nossos resultados mostram que estes cenários oferecem diferentes graus de proteção, alterando a vulnerabilidade do território, a estrutura da paisagem e a representatividade dos alvos de proteção selecionados. $\mathrm{O}$ Cenário CDB 4, que combina políticas de comando e controle e de governança ambiental, pode proteger frações representativas do Cerrado, as grandes áreas remanescentes ao norte e as porções mais fragmentadas ao sul. Mas a implementação dessas ações demandaria a complementação do sistema de áreas protegidas aliadas a estratégias que busquem a conectividade de áreas com alto índice de fragmentação.
\end{abstract}

PALAVRAS-CHAVE: Mudanças no uso e cobertura da terra; Convenção sobre Diversidade Biológica; desmatamento; cenários; Cerrado. 


\section{INTRODUÇÃO}

A Conferência das Partes (COP), órgão da Convenção sobre Diversidade Biológica (CDB), estabeleceu em sua 10a reunião em Aichi, ocorrida em 2010, várias metas para até 2020, com o objetivo de reduzir as pressões sobre os ecossistemas e buscar a proteção da biodiversidade (CDB, 2013; Brasil, 2010b). A meta 5 objetiva a redução da perda, degradação e fragmentação dos hábitats naturais e a meta 11 , estabelece percentuais de ecossistemas a serem protegidos, por meio de áreas protegidas, considerando a sua representatividade e conectividade (CDB, 2013). Uma das mais importantes estratégias de conservação é a criação de áreas protegidas, talvez a mais efetiva para a proteção da biodiversidade (CDB, 1992; Bruner et al., 2001; Lawler et al., 2003; Kharouba \& Kerr, 2010, Carranza et al., 2014; Carranza et al, 2014b) e o foco da maioria das agências que promovem a conservação no mundo (Baudron \& Giller, 2010). Por isso, é importante que essas áreas protegidas sejam representativas quanto à biodiversidade que protegem (Lawler et al., 2003).

O conceito de representatividade implica que a biodiversidade regional deva ser conservada nos seus diferentes níveis organizacionais (Noss, 1990; CDB, 1992). A partir de recomendações decorrentes da COP-7/CDB (UNEP-CDB, 2004) o governo brasileiro passou a utilizar a identificação sistemática de prioridades espaciais para a conservação da biodiversidade (Brasil, 2004b; Brasil, 2007; Brasil, 2007b). Tal abordagem consta na Política Nacional de Biodiversidade (Brasil, 2002) e, a partir de critérios de representatividade, tem o objetivo de identificar áreas específicas para a complementação do sistema de áreas protegidas (Brasil, 2000; Weigand Jr et al., 2011). A definição de áreas prioritárias a partir do uso da complementaridade é bastante relevante e essa importância é corroborada pelo estudo de Rodrigues et al. (2004). Os autores estimaram que $12 \%$ da diversidade de espécies de distribuição restrita e ameaçadas de extinção não estão protegidas por qualquer área protegida (Rodrigues et al., 2004), que por sua vez, representam $13 \%$ das áreas terrestres (CDB, 2013).

Além da representatividade, a persistência de processos biológicos e espécies também deve ser considerada em estudos para o planejamento da conservação (Cowling et al. 1999; Margules $\&$ Pressey, 2000; Gaston et al., 2002; Pressey et al., 2007). A vulnerabilidade é a probabilidade ou iminência de perda de biodiversidade causada por ameaças antrópicas ou naturais (Pressey \& Taffs, 2001). A análise da vulnerabilidade tem por objetivo minimizar os riscos aos quais elementos da biodiversidade estão expostos, garantindo assim, a persistência das espécies ou a viabilidade de populações (Margules \& Pressey, 2000; Pressey \& Taffs, 2001; Gaston et al., 2002; Lawler et al., 2003; Shi et al., 2005). Ela pode ser analisada sob a óptica das mudanças climáticas (Marini et al., 2009; Kharouba et al., 2010) ou mesmo considerando a invasão de espécies exóticas (Rouget et al., 2003). Contudo, a perda e a fragmentação de hábitats (Fahrig, 2003; Li et al., 2010) 
convertidos para uso antrópicos (Verburg et al., 2006; Pressey et al., 2007; UNEP, 2012), talvez sejam as mais prementes ameaças à conservação da biodiversidade (Gaston et al., 2002). A abordagem mais conhecida de priorização de áreas, envolvendo o conceito de vulnerabilidade é aquela relativa aos "Hotspots da biodiversidade” (Myers et al., 2000; Mittermeier et al, 2004), que são áreas com alto endemismo de plantas e com grandes taxas de conversão do solo para uso antrópico.

Apesar do Brasil ter sido um dos primeiros signatários da Convenção sobre Diversidade Biológica - CDB (CDB, 2015) e possuir uma ampla legislação ambiental que atende em grande parte aos objetivos da CDB, ainda enfrenta intensas contradições internas, marcadas pela forte tensão entre os segmentos conservacionista e ruralista. De acordo com Teixeira (2012), entretanto, o modo com que as políticas ambientais no país vêm sendo conduzidas parece estar em oposição aos compromissos internacionais assumidos pelo Brasil.

A fronteira agrícola brasileira teve uma rápida expansão, a partir dos anos 70 (Aguiar \& Monteiro, 2005) e essa situação foi mais drástica no Cerrado, onde as taxas de desmatamento foram impulsionadas pela agricultura mecanizada de soja (Smith et al., 1998; Silva et al., 2006; Jepson et al., 2010). Apesar das altas taxas de conversão dos biomas brasileiros, observa-se uma tendência na diminuição das taxas de desmatamento (Brasil, 2011), que pode estar sendo influenciada pela efetividade de algumas ações de conservação, como os Planos de Ação para a Prevenção e Controle do Desmatamento - Amazônia \& Cerrado (Brasil, 2015), a moratória da soja (Barreto et al., 2013, Arima et al., 2014) e a criação de áreas protegidas (Verburg et al., 2006), pelos investimentos tecnológicos no uso da terra pelo agronegócio (Barreto et al., 2013) e pela queda no preço das commodities agrícolas no mercado internacional (Killeen et al., 2007, Jepson et al., 2010, Arima et al., 2014, Verburg et al., 2014).

O conceito de vulnerabilidade que utilizamos nesse artigo parte da compreensão da dinâmica do uso do solo e cobertura da terra (Land Use Cover Change - LUCC). A vulnerabilidade deve ser avaliada tanto na escala espacial, como na temporal (Gaston et al., 2002; Pressey et al., 2007), dada as altas taxas de mudanças na paisagem. Assim, é provável que o problema da manutenção da persistência das populações e ecossistemas não possa ser resolvido se considerado o cenário atual de ameaças (Lawler et al., 2003; Pérez-Vega et al., 2012). Por isso, os modelos de simulação dinâmica, são importantes instrumentos, ao auxiliar na compreensão dos processos naturais e antropogênicos como agentes de mudanças nos ecossistemas, antecipando os possíveis caminhos da evolução do espaço geográfico (Callicott et al., 2007). Por meio dos modelos de LUCC, os formuladores de políticas e gestores podem analisar padrões e projetar cenários que mostrem como decisões políticas, econômicas e sociais afetarão no futuro o sistema retratado (Mas et al., 2005; Callicott et al., 2007; Abreu et al., 2011; Pérez-Vega et al., 2012; Müller et al., 2013). 
A definição de indicadores ambientais (Brasil, 2014), seu monitoramento (Balmford et al., 2005) com a produção de séries de dados temporais e disponibilização desses dados, juntamente com o estabelecimento de metas realistas e mensuráveis, do desenvolvimento de ferramentas de sensoriamento remoto e dos modelos quantitativos ecológicos e de uso da terra são instrumentos que, alinhados, podem auxiliar os tomadores de decisão a conduzir as políticas públicas no país. No Brasil, o governo federal passou a monitorar o desmatamento da cobertura vegetal como uma forma de avaliar a efetividade de alguns dos objetivos assumidos perante a CDB (Brasil, 2000; UNEP-CDB, 2004) e como as atuais políticas ambientais poderão afetar a conservação da biodiversidade. Assim, a partir de 2008, séries temporais tornaram-se disponíveis indicando as taxas de desmatamento em cada um dos biomas brasileiros (Brasil, 2011). Com base nesses dados, é possível estabelecer cenários tendenciais ou prognósticos de perda de áreas para os biomas já monitorados.

Ainda há pouco acúmulo de conhecimento sobre esse tema no Brasil. No Cerrado, alguns estudos buscaram entender como as forçantes socioeconômicas e políticas atuam na evolução do uso e ocupação do solo e, para isso, utilizaram projeções estatísticas e modelagens espaciais (Klink \& Machado, 2005; Jepson et al., 2010; Ferreira et, 2012; Salmona; 2013; Faleiro \& Loyola, 2013; Faleiro \& Loyola, 2013b; Grecchi et al., 2014; Stan et al., 2015; Blauchle et al., 2015).

O conjunto de metas do governo brasileiro (Tab. S1) pode orientar as políticas públicas quando estabelecidas por meio de critérios técnicos e científicos, mas se forem baseadas somente em argumentos políticos ou limiares padrão, podem acabar desviando os objetivos da conservação (Pressey et al., 2003; Svancara et al., 2005). O objetivo do nosso trabalho foi testar a eficácia de algumas das metas estipuladas pelo Brasil no âmbito da CDB para a conservação da biodiversidade do Cerrado, particularmente em relação à sua representatividade e persistência. Para tanto, foram utilizados modelos da dinâmica de LUCC para o bioma, modelos esses baseados nos cenários Business as usual - BAU (manutenção das atuais tendências de desmatamento) e de cenários "GOV", construídos de acordo com algumas das metas estipuladas pelo Brasil para a CDB. Os modelos projetaram as mudanças no uso da terra por um período de 40 anos. Para avaliar a persistência, analisamos os remanescentes do bioma Cerrado, de acordo com o tempo pretérito, atual e em cenários futuros. Com isso foi possível uma avaliação da evolução das métricas da paisagem nos cenários propostos e ainda as metas alteram a vulnerabilidade dos ecossistemas. Em relação à representatividade ecossistêmica do bioma, analisamos as lacunas de conservação dos alvos (Jennings, 2000) selecionados nos cenários propostos, avaliando como as estratégias propostas pelo Brasil podem alterar esse modelo.

Como premissa, assumimos que o cenário futuro de BAU segue a tendência de desmatamento observada mais recentemente, levando em conta os arranjos da paisagem já consolidados e que 
portanto, não incorpora alterações na governança previstas pela CDB. Nesse contexto, pergunta-se: as metas estipuladas por ocasião da CDB serão realistas e, portanto, suficientes para alterar este modelo? A hipótese é de que a aplicação das metas assumidas pelo Brasil perante a CDB também não serão suficientes para alterar o modelo atual de uso do solo e que, para garantir a persistência e representatividade da biodiversidade, serão necessários esforços de conservação diferenciados. Assim, utilizamos o caso do bioma Cerrado, que possui grande heterogeneidade espacial de sua biodiversidade (Ratter \& Dargie 1992; Silva et al., 2006; Brannstrom et al., 2008) e discrepantes modelos de ocupação antrópica em suas diferentes porções (Machado et al., 2004; Brannstrom et al., 2008; Jepson et al., 2010).

\section{METODOLOGIA}

\section{1. Área de estudo}

O Cerrado, correspondente a uma área de 2 milhões de $\mathrm{Km}^{2}$ (Brasil, 2004), é o segundo maior bioma brasileiro em extensão territoral e está localizada na porção central do Brasil. O bioma guarda uma grande diversidade de tipos de solos, geologia, geomorfologia e clima (Silva et al., 2006), vegetação savânica, campestre e ambientes florestais (Ratter et al., 1997; Oliveira-Filho \& Ratter, 2002). Considerado um hotspot mundial para a biodiversidade (Mittermeier et al., 1998; Myers et al., 2000; Mittermeier et al., 2004), tem distribuição espacial da vegetação natural altamente heterogênea e os maiores fragmentos remanescentes estão localizados principalmente em sua parte norte (Sano et al., 2010). A ocupação comercial do Cerrado foi iniciada nos anos 20 com a cultura do café e, hoje, é uma importante frente de expansão da agricultura mecanizada. Uma análise da dinâmica de uso do solo mostra a expansão e a retração dos plantios de soja, milho, algodão e cana-de-açúcar, e também no número de cabeças de gado. Alguns autores sugerem a existência de um fenômeno de substitução de uma cultura ou atividade econômica pela outra provocando o deslocamento das atividades substituídas para áreas de vegetação nativa (Brandão et al., 2006; Rêdo et al., 2013, Grecchi et al., 2014). Mas, de fato, estudos que analisaram o uso e ocupação do Cerrado mostram que as principais causas de desmatamento, são a agricultura mecanizada e as pastagens para forrageio bovino (Alho \& Martins, 1995; Smith et al., 1998; Klink \& Moreira, 2002; Klink \& Machado, 2005; Jepson, 2005; Brannstron et al., 2008; Jepson et al., 2010). Atualmente, o Cerrado ainda é um dos biomas brasileiros mais ameaçados. Em 2011 sua área remanescente de vegetação ocupava cerca de $50 \%$ e a taxa de desmatamento anual é 7.246,67 $\mathrm{Km}^{2}$ ano $^{-1}$, a maior taxa dentre os seis biomas brasileiros (Brasil, 2011). Além disso, as taxas de desmatamento do bioma são bastante variáveis entre as suas porções (Brannstrom et al., 2008). 


\subsection{Construção do modelo de transição potencial}

Nós elaboramos os modelos de uso do solo utilizando o algoritmo Land Change Modeler for Ecological Sustainability (LCM) do programa Idrisi 16.05 (Eastman, 2009). Esta plataforma usa como entrada principal um mapa de paisagem e as variáveis espaciais, estruturadas em dois subconjuntos cartográficos, de acordo com sua natureza dinâmica ou estática. Como saída, são gerados mapas de paisagem simulados e multitemporais, os quais indicam a probabilidade de uma célula em uma posição, mudar de estado para estado. O algoritmo utilizado foi o Multi-Layer Perceptron (MLP), que é uma rede neural que utiliza complexas funções não-lineares para encontrar a relação entre mudanças do uso do solo e suas variáveis espaciais (Eastman, 2009). Assim, calculamos as probabilidades de mudanças entre as classes de uso do solo (cerrado para antrópico) a partir de mapas de remanescentes da cobertura vegetal brasileira: um mapa representando $\mathrm{t}_{0}$, ano de 2002 (PROBIO/MMA, 2007, disponível em http://mapas.mma.gov.br/mapas/aplic/probio/datadownload.htm) e $\mathrm{t}_{1}$, ano de 2010 (PMDBBS IBAMA/MMA, 2012 disponível em http://siscom.ibama.gov.br/monitorabiomas/) contendo as alterações na paisagem.

Nesse modelo, utilizamos as seguintes variáveis espaciais: estáticas (altimetria, declividade, pluviosidade e distância de estradas) e dinâmicas (distância dos desmatamentos: 2002) (Tab. S2). Essas variáveis foram selecionadas quanto ao seu poder de explicação e utilizamos aquelas que apresentaram um valor superior a 0,12 para a estatística V de Cramer (Eastman, 2009). As variáveis também analisadas quanto à sua redundância, pela função Análise de Componentes Principais (PCA) fator de colinearidade < 0,6, todas ajustadas e formatadas em Salmona (2013). Essas variáveis e a transição de uso do solo entre $t_{0}$ e $t_{1}$, foram inseridas no modelo para subsidiar as redes neurais e gerar a Cadeia de Markov, que calcula a probabilidade de mudança entre as classes de uso do solo. Os cenários futuros foram projetados a partir desse modelo. A validação do modelo seguiu os procedimentos dados por Salmona (2013) que por sua vez observou a metodologia de Pontius Jr (2004). A abordagem compara o resultado da previsão com um modelo nulo e analisa-o em múltiplas resoluções (ver Salmona, 2013 para detalhes). Como resultado desse processo, criamos um mapa de transição potencial, que aloca as probabilidades de transição de uma classe de uso do solo para outra, neste caso, de cerrado para antrópico. Essas probabilidades de transição foram utilizadas como um mapa de vulnerabilidade ao desmatamento. Todos as imagens foram padronizadas para a resolução de $0,5 \mathrm{Km}$, com sistema de coordenadas geográficas: GCS-Sirgas 2000. Outros parâmetros utilizados para a construção do modelo de transição potencial estão descrito em material suplementar (Fig. S1) 


\subsection{Desenho conceitual e elaboração dos cenários}

Nós elaboramos cenários Business as usual - BAU, ou "o mesmo de sempre" (Soares-Filho et al., 2006) e 4 cenários que consideram a governança ambiental (GOV.), estes últimos concebidos para testar as metas acordadas pelo governo em virtude da Convenção sobre Diversidade Biológica (CDB) (Tab. 2).

Tab. 2: Descrição dos cenários elaborados neste estudo.

\begin{tabular}{|c|c|}
\hline CENÁRIOS & DESCRIÇÃO \\
\hline BAU & $\begin{array}{l}\text { As taxas de desmatamento tendem a continuar fora das Unidades de Conservação de Proteção Integral } \\
\text { (Categorias 1-IV da IUCN) e Terras Indígenas e mantêm suas tendências históricas nas Unidades de } \\
\text { Conservação de Uso Sustentável; novas unidades de conservação não serão criadas; nenhuma nova } \\
\text { política pública será planejada visando conciliar o desenvolvimento com a conservação do meio } \\
\text { ambiente, as metas assumidas pelo governo perante a CDB não são consideradas nas mesas de negociação } \\
\text { setoriais. }\end{array}$ \\
\hline $\begin{array}{l}\text { CDB } 1 \text { (Aumento da } \\
\text { governança) }\end{array}$ & $\begin{array}{l}\text { Cumprimento das metas brasileiras (BRASIL, 2006), elaboradas no âmbito da Meta } 11 \text { (AICHI, 2011), } \\
\text { onde está previsto que a "proteção da biodiversidade deverá ser assegurada em pelo menos } 2 / 3 \text { das Áreas } \\
\text { Prioritárias para Biodiversidade por meio de Unidades do SNUC, Terras Indígenas e Territórios } \\
\text { Quilombolas". As taxas de desflorestamento tendem a continuar fora das Unidades de Conservação de } \\
\text { Proteção Integral e Terras Indígenas e mantêm suas tendências históricas nas Unidades de Conservação } \\
\text { de Uso Sustentável. Novas Unidades de Conservação serão criadas em } 2 / 3 \text { das áreas consideradas } \\
\text { prioritárias para a conservação da biodiversidade, de acordo com sua importância biológica e } \\
\text { vulnerabilidade ao desmatamento. }\end{array}$ \\
\hline $\begin{array}{l}\text { CDB } 2 \text { (Aumento da } \\
\text { governança) }\end{array}$ & $\begin{array}{l}\text { Cumprimento da Meta } 11 \text { (AICHI, 2011) "17\% das áreas terrestres, especialmente áreas de particular } \\
\text { importância para a biodiversidade e serviços ecossistêmicos, devem estar satisfatoriamente } \\
\text { interconectadas e conservadas pelo Sistema Nacional de Áreas Protegidas". As taxas de desmatamento } \\
\text { tendem a continuar fora das Unidades de Conservação de Proteção Integral e Terras Indígenas, mantendo } \\
\text { suas tendências históricas nas Unidades de Conservação de Uso Sustentável. Novas Unidades de } \\
\text { Conservação serão criadas em } 17 \% \text { das áreas representativas da vegetação, de acordo com sua } \\
\text { importância biológica e vulnerabilidade. }\end{array}$ \\
\hline $\begin{array}{l}\text { CDB } 3 \text { (Comando e } \\
\text { Controle) }\end{array}$ & $\begin{array}{l}\text { Cumprimento da meta brasileira (BRASIL, 2006) e Meta } 5 \text { (AICHI, 2011), redução na taxa de perda de } \\
\text { todos os hábitats naturais, incluindo as florestas, de pelo menos } 50 \% \text {, e se possível levado para perto de } \\
\text { perda zero (para 2020) e consequente redução significativa da fragmentação. As taxas de desmatamento } \\
\text { devem ser reduzidas em } 50 \% \text { fora das Unidades de Conservação de Proteção Integral e também devem } \\
\text { ser reduzidas nesta mesma taxa no interior das Unidades de Conservação de Uso Sustentável e Terras } \\
\text { Indígenas. Políticas públicas de comando e controle serão intensificadas, mas nenhuma nova Unidade de } \\
\text { Conservação será criada no período. }\end{array}$ \\
\hline $\begin{array}{l}\text { CDB } 4 \text { (Aumento da } \\
\text { Governança e } \\
\text { Comando e } \\
\text { Controle) }\end{array}$ & $\begin{array}{l}\text { Este cenário busca a combinação de políticas de comando e controle (descritas no Cenário CDB } 3 \text { ) e } \\
\text { governança ambiental (Cenários CDB } 1 \text { e } 2 \text { ). }\end{array}$ \\
\hline
\end{tabular}

Para gerar os cenários utilizamos dois recursos do LCM: alteração das taxas de probabilidade de desmatamento da cadeia de Markov, no Change Demand Modeling e em Constraints and Incentives (opção do programa para impor restrições espaciais aos cenários), foram inseridas camadas de restrição (de acordo com os cenários descritos) que promovem a inibição total à conversão do uso do solo. O módulo Change Prediction do LCM foi utilizado para gerar os cenários futuros: os mapas de remanescentes (hard prediction) e outro mapa de vulnerabilidade ao desmatamento (soft prediction).

Todos os cenários têm como camada de restrição Unidades de Conservação de Proteção Integral e Terras Indígenas (default). O conjunto de tais áreas para o Cerrado perfaz um total de 237 áreas protegidas, com área de $142.000 \mathrm{Km}^{2}$ (Brasil, 2013b). A opção pela inclusão dos territórios indígenas justificou-se pelo fato de serem computadas pelo Brasil para cumprimento 
das metas para a CDB. Pelo mesmo motivo, deveríamos ter utilizado os Territórios Quilombolas, mas neste caso não houve a inclusão, pelo fato de não haver mapeamentos disponíveis dessas áreas.

Para a criação do cenário BAU (Fig. 2A) utilizamos a camada de restrição default mantendose as mesmas taxas de desmatamento calculadas automaticamente pelo LCM.

Para o Cenário CDB1 (Fig. 2B), utilizamos a camada de restrição default acrescida de 2/3 das Áreas Prioritárias para a Conservação da Biodiversidade do Cerrado (Brasil, 2013b), descontadas aquelas já protegidas pelas Unidades de Conservação de Proteção Integral e Terras Indígenas. Para a seleção dos $2 / 3$ das áreas prioritárias a serem incluídas como camada de restrição, utilizamos os conceitos de insubstituibilidade e vulnerabilidade (Pressey \& Taffs, 2001; Pressey et al., 2007). Em primeiro lugar foram selecionadas todas aquelas áreas com maior prioridade e, portanto, maior valor para a conservação, ou com maior insubstituibilidade. Depois, como critério de desempate, utilizamos o conceito de vulnerabilidade que, neste caso, significou a maior probabilidade de conversão de áreas naturais em antrópicas, entre o período de 2002 e 2010 abordagem reativa (Brooks et al., 2006) (Fig.1). Esse mapa foi produzido em etapa anterior pelo modelo LCM. Assim, das 289 áreas prioritárias, foram selecionadas 157, com área total de $231.902 \mathrm{Km}^{2}$ o que representa $11,37 \%$ da área total do Cerrado.

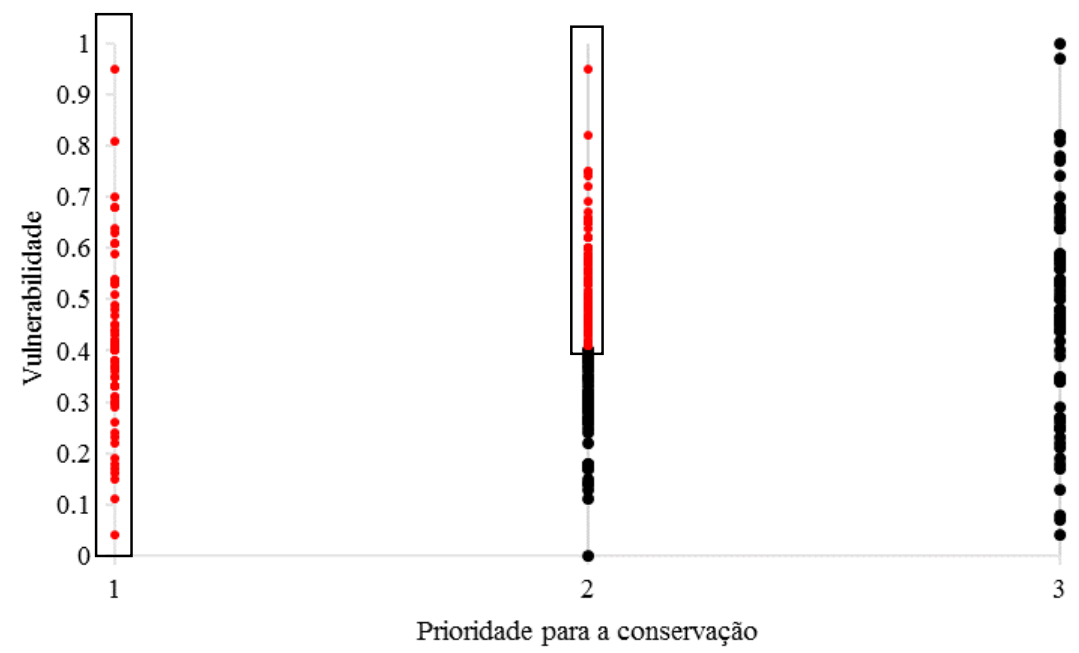

Fig. 1: Análise de vulnerabilidade versus prioridades para a conservação: os símbolos em preto representam 289 áreas prioritárias e, em vermelho, são aquelas selecionadas para compor o CDB1.

Para o Cenário CDB2 (Fig. 2C), que busca cumprir as metas acordadas em Aichi, 28 classes de vegetação natural (Brasil, 1993) foram utilizadas como substitutos das "áreas terrestres, especialmente áreas de particular importância para a biodiversidade e serviços ecossistêmicos" (Tab. S3). A meta de 17\% dessas áreas foi calculada de acordo com a cobertura pretérita do Cerrado (Brasil, 2010) (Fig.S3). Para desenhar a camada de restrição deste cenário, utilizamos o 
programa MARXAN (Ball et al, 2000; Ball et al., 2009), para selecionar áreas que representem as metas propostas, mantendo a coesão espacial das unidades, minimizando os custos de implementação do sistema e complementando as áreas protegidas já existentes. Para facilitar a integração das informações a área de estudo foi dividida em 19.975 Unidades de Planejamento (UPs): hexágonos de $100 \mathrm{Km}^{2}$ de área e Unidades de Proteção Integral e Terras Indígenas.

A probabilidade de conversão de áreas naturais em antrópicas, entre o período de 2002 e 2010, foi utilizada como custo no programa MARXAN. Esses valores de probabilidade foram padronizados para que o maior valor fosse 1 e depois invertidos. Então, quanto maior a vulnerabilidade da área, menor seu valor de custo, uma vez que o programa prioriza as soluções com menor custo. O MARXAN também permite ajustar a aglutinação das áreas selecionadas, por meio de um peso de borda (Boundary Lenght Modifier - BLM). Para definir a borda utilizamos a metodologia sugerida por Stewart \& Possingham (2005). O programa também foi calibrado para considerar as Unidades de Proteção Integral e Terras Indígenas como já protegidas. Além disso, foram indicadas UPs que coincidiam com as Áreas Prioritárias para a conservação da biodiversidade (Brasil, 2013b), como áreas preferenciais na solução final. Os demais parâmetros estão descritos em material suplementar (Fig. S2). Utilizamos o melhor modelo do MARXAN (Best Solution) para a construção da camada de restrição.

Para o Cenário CDB3 (Fig. 2A), reduzimos pela metade as taxas de desmatamento calculadas pela cadeia de Markov, e a camada de restrição utilizada é a mesma do cenário BAU.

O Cenário CDB4 (Fig. 2D) prevê a combinação das metas assumidas pelos CDB1, CDB2 e CDB3. Para tanto, utilizamos o MARXAN para complementar as áreas selecionadas pelo cenário CDB1, de modo que as metas do cenário CDB2 também fossem atingidas. No MARXAN indicamos que as Áreas Prioritárias para a Conservação da Biodiversidade, selecionadas em CDB1, deveriam necessariamente constar da solução final. O valor de borda foi zero para que não fosse gerada uma solução com áreas muito extensas. Os demais parâmetros foram os mesmos descritos no cenário CBD2. Assim, a camada de restrição deste cenário é o produto desse processamento acrescido da camada default. As taxas de desmatamento calculadas automaticamente pela cadeia de Markov, do LCM, também foram reduzidas pela metade. Os demais parâmetros estão descritos em material suplementar (Fig. S2). 

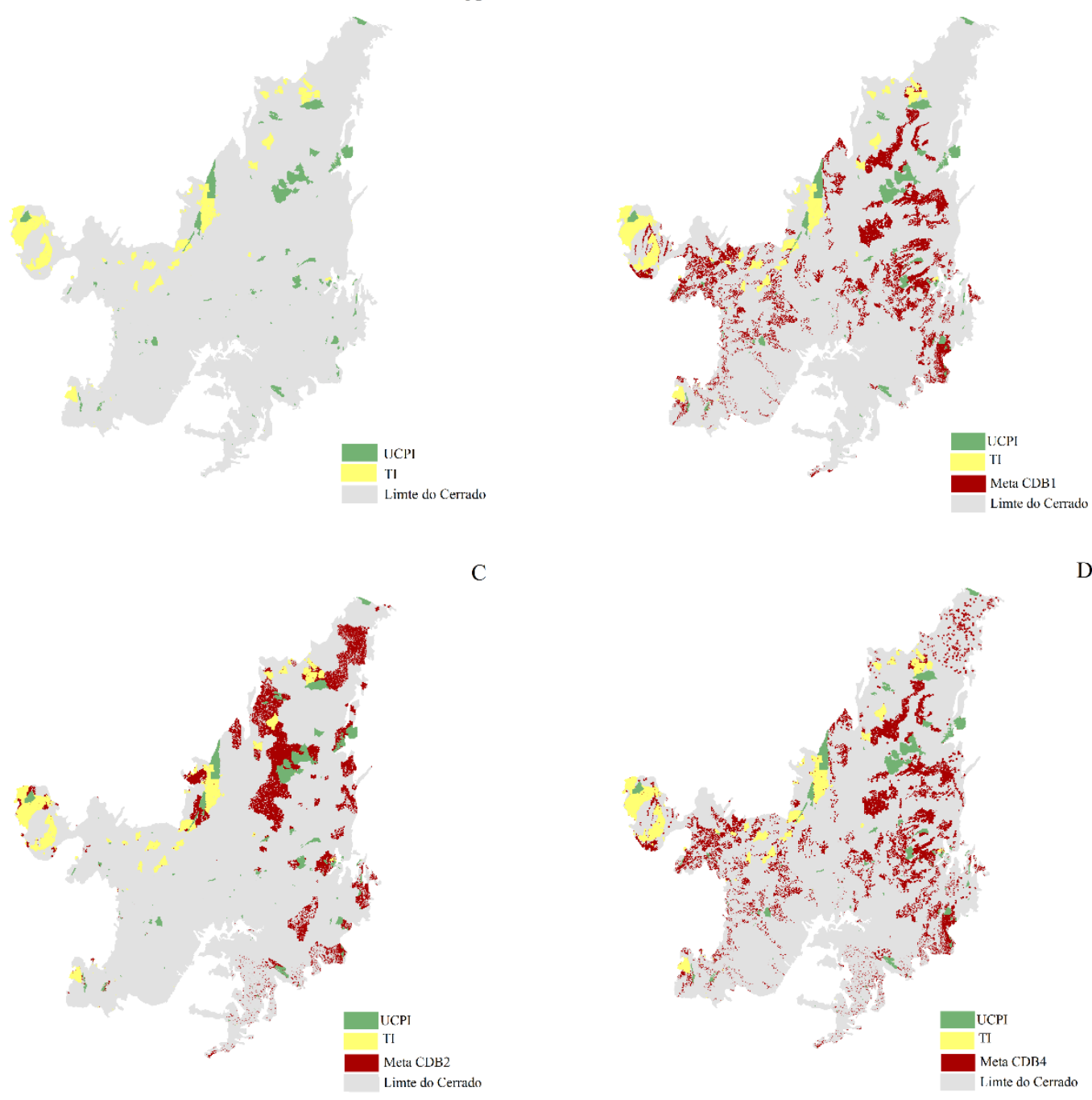

$\mathrm{C}$

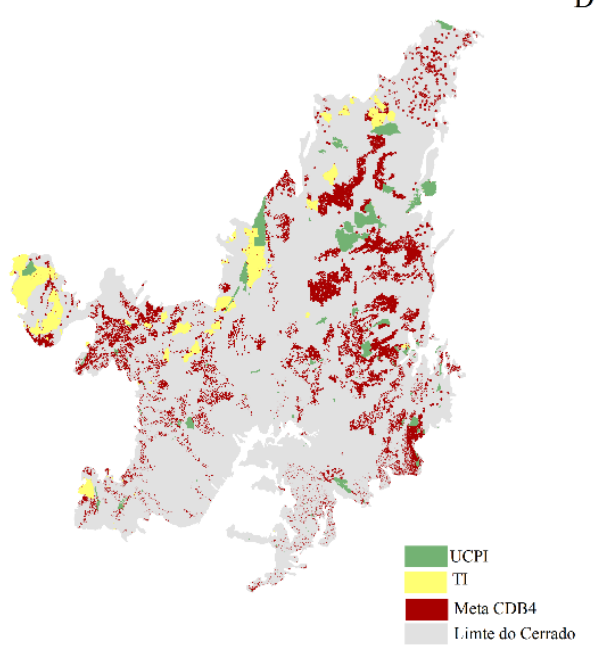

Fig. 2: Camadas de restrição para os cenários: A- cenários BAU e GOV. (CDB3), de B - D cenários GOV. (CDB1, CDB2 e e CDB4, respectivamente).

\subsection{Análises dos cenários}

Comparamos a perda quantitativa de potenciais hábitats entre os tempos pretéritos (1988 a 1994, MCTI; 1995 a 2001, Funcate; 2002 a 2010, IBAMA/MMA) e os cenários futuros, BAU e CDB3 e CDB4. Para inferir sobre a conversão do uso do solo nos diferentes cenários (BAU e GOV.), foi calculado o Kappa Index of Agreement - KIA (Pontius, 2000), utilizando o algoritmo Crosstab do software Idrisi 16.05 (Eastman, 2009). Segundo esse cálculo, índices próximos a 1 indicam correlação total na comparação entre dois mapas.

Utilizamos as métricas da paisagem (McGarigal \& Marks, 1995) para identificar tendências e padrões de modificação da estrutura da paisagem. Tal abordagem permite inferir como os cenários propostos contribuíram para garantir a persistência e funcionalidade dos ecossistemas do 
Cerrado. As métricas de paisagem foram calculadas utilizando as rotinas ClassStat e PatchStat do pacote SDMTools (Vanderwal et al., 2014) do programa R (R Core Team, 2012), para o bioma:

1. Métricas de fragmentos: área total (TA), número de fragmentos (NP), densidade de fragmentos (PD), tamanho médio dos fragmentos (MPS), área máxima do fragmento (MaxPA), índice do maior fragmento (LPI), desvio-padrão da área dos fragmentos (PSSD), tamanho dos fragmentos;

2. Métrica de borda: total de bordas (TE);

3. Métricas de forma: índice de forma da paisagem (LSI);

4. Métricas de área central: área central total (TCA);

5. Métricas de agregação: índice de agregação (AI); índice de divisão da paisagem (Division), índice de divisão (Split), tamanho efetivo da malha (Mesh).

Fizemos uma análise exploratória das variáveis usando uma Análise de Componentes Principais (PCA) com matriz de correlação e os dados em escala logarítmica. Em seguida, selecionamos as métricas representadas pelos primeiros componentes da PCA, revelando aquelas que explicam a maior variabilidade de padrões da paisagem. Utilizamos a rotina ggbiplot do pacote devtools do programa R (R Core Team, 2012) para essas análises.

Para observar padrões de desmatamentos futuros acumulados (de 2010 a 2050), fizemos uma interpolação com um polinômio de 9a. Ordem, utilizando o Trend Surface Analysis of Change do Idrisi. Para avaliar a vulnerabilidade ao desmatamento dos alvos do estudo, compilamos os valores médios de probabilidade de desmatamento extraídos das camadas soft preditions para cada um dos cenários propostos, por cada uma das 28 classes de vegetação (Brasil, 1993). Também fizemos uma análise agrupando as classes de vegetação (Brasil, 1992) por formação ou áreas de tensão ecológica: ecótonos ou encraves, florestas estacionais, formações pioneiras e savanas. Para esses cálculos, nós utilizamos zonal statistics do ArcGis 10.3.1 (Esri, 2015).

Para avaliar a representatividade dos alvos (28 classes de vegetação - Brasil, 1993) (Tab. S3), combinamos a vegetação pretérita (Brasil, 2010) (Fig. S3) e os remanescentes contidos nos cenários gerados, a cada 10 anos. Consideramos como lacunas de representação, os alvos com áreas menores que 17\% de sua área pretérita. Procedemos as análises no Image calculator (Idrisi).

\section{RESULTADOS}

\subsection{As taxas de desmatamento do Cerrado}

As taxas históricas de desmatamento do Cerrado, fornecem um panorama sobre a evolução do uso e ocupação do solo do bioma e também sobre o cumprimento das metas de conservação estabelecidas. A Fig. 3 mostra essa evolução e o prognóstico futuro de desmatamento, 
considerando os cenários $\mathrm{BAU}$ e CDB3 ou CDB4, que preveem uma redução de $50 \%$ nas taxas de desmatamento calculadas entre os anos de 2002 e 2010. A meta de desmatamento anual deverá ser $\leq 7.637 \mathrm{Km}^{2}\left(0,36 \%\right.$ ano $^{-1}$, taxa bruta $)$ ou de $\left(0,735 \%\right.$ ano $^{-1}$, taxa líquida $)$ no âmbito da CDB (Brasil, 2013c), e para o clima (Política Nacional sobre Mudança do Clima - PNMC), $\leq$ de 7.851 $\mathrm{Km}^{2}\left(0,37 \%\right.$ ano $^{-1}$ taxa bruta), calculada em relação à média de 1999 a 2008 , ou $15.700 \mathrm{~km}^{2}$ (Brasil, 2010c). O cenário BAU mostra que essas metas não deverão ser atingidas e que, em 2050, essa taxa permanecerá em 8.979,18 Km². No Cenário CDB3, essa meta seria atingida já em 2020, tendendo ao decréscimo até o ano de 2050 (Fig. 4). Mesmo mantendo-se dentro das metas estabelecidas pela $\mathrm{CDB}$, como mostram os últimos dados de desmatamento publicados (Brasil, 2011), o Cerrado teria em 2020 uma área remanescente $1.023 .8493 \mathrm{Km}^{2}$, ou menos de $50 \%$ de sua vegetação remanescente e, em 2050 , teria perdido mais de $60 \%$ de sua vegetação original (Fig. 5).

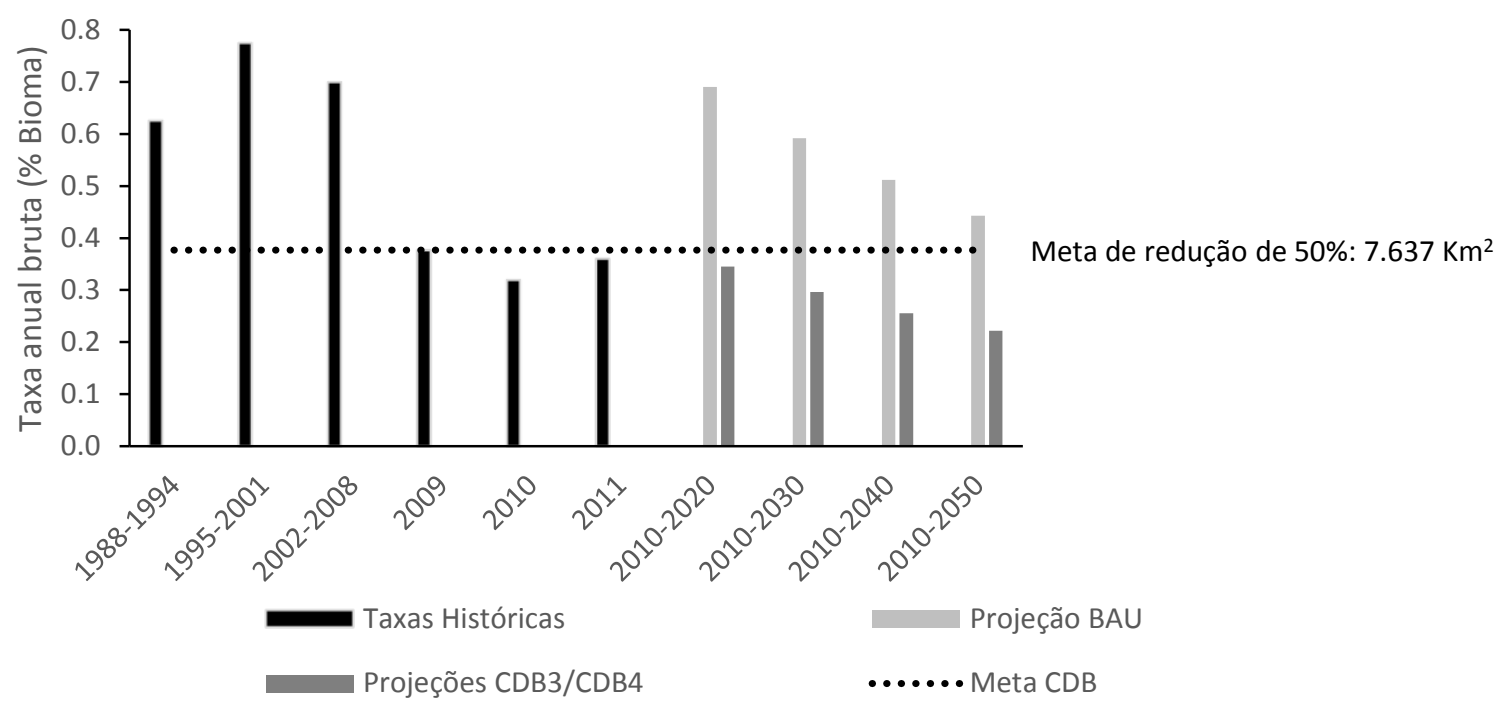

Fig. 3: Taxas históricas de desmatamento do Cerrado (Fontes: 1988 a 1994, MCTI; 1995 a 2001, Funcate; 2002 a 2008, MMA; 2009 e 2011, IBAMA/MMA) e Projeção cenários BAU e CDB3); Metas de redução do desmatamento, segundo o plano nacional apresentado à CDB (Brasil, 2013c). 


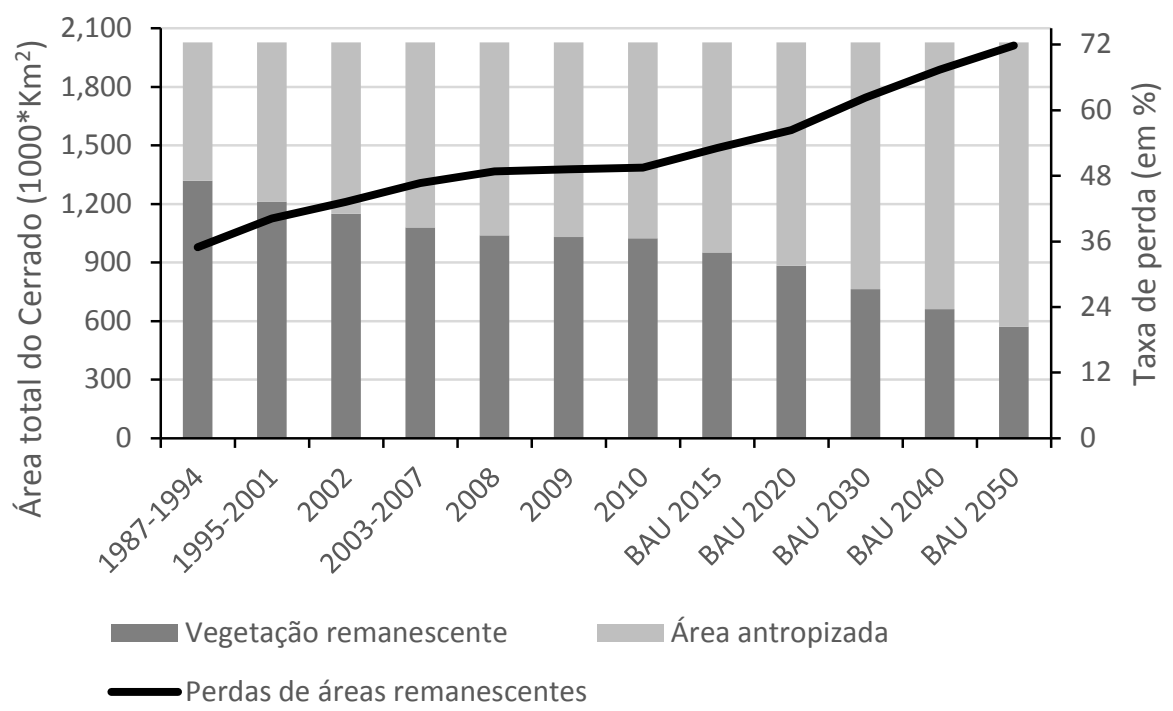

Fig. 4: Série histórica de desmatamento do Cerrado (Fontes: 1987 a 1994, MCTI; 1995 a 2001, Funcate; 2002 a 2008, MMA; 2009 e 2010, MMA/IBAMA), os valores absolutos foram calculados com base nas taxas médias retroativas de desmatamento) e Projeção Cenário BAU.

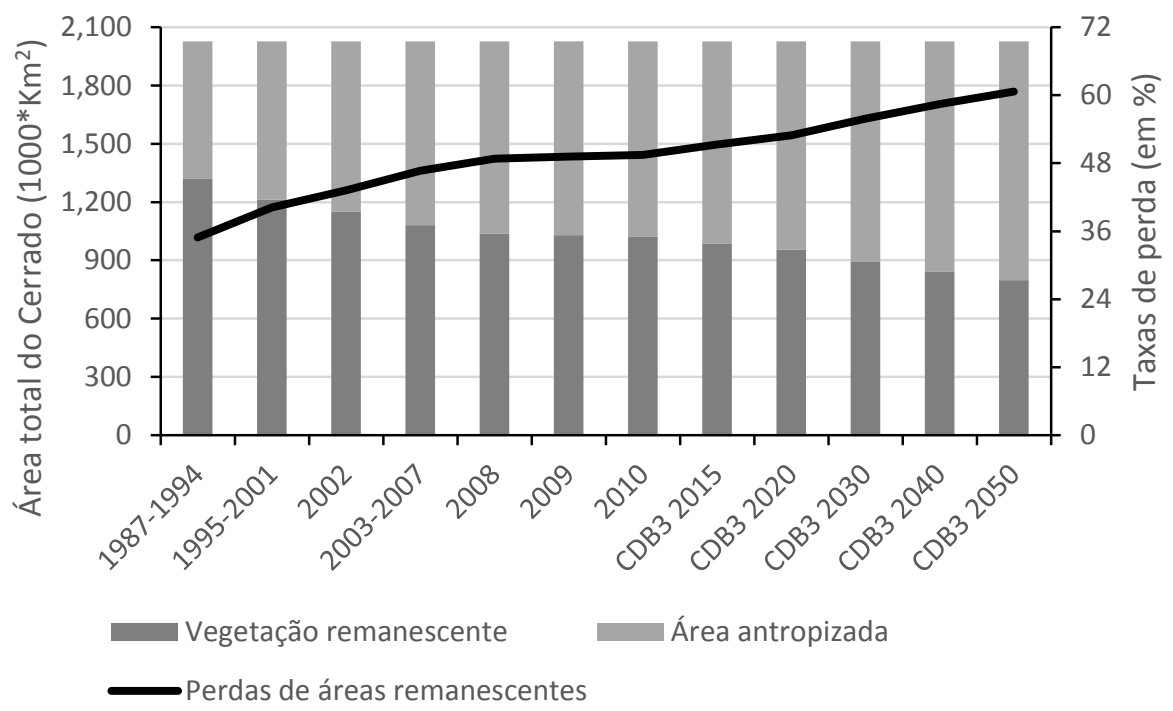

Fig. 5: Série histórica de desmatamento do Cerrado (Fontes: 1987 a 1994, MCTI; 1995 a 2001, Funcate; 2002 a 2008, MMA; 2009 e 2010, MMA/IBAMA), os valores absolutos foram calculados com base nas taxas médias retroativas de desmatamento) e Projeção Cenário CDB3. 


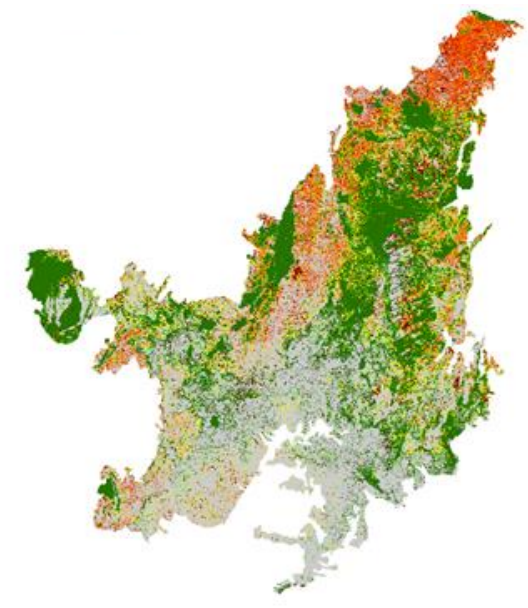

A
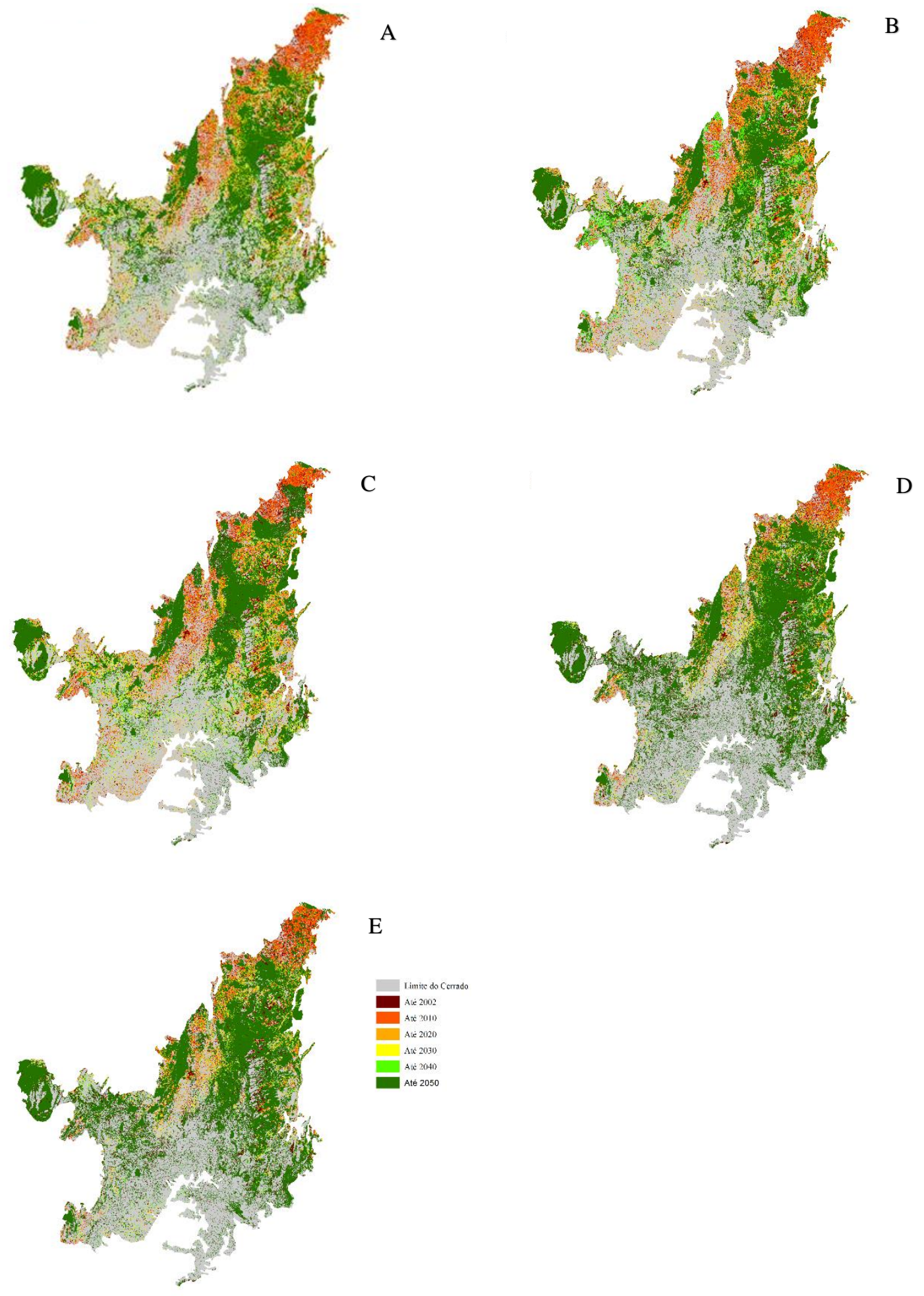

Fig. 6: Evolução do desmatmento do Cerrado em cada um dos cenários: A- BAU, B - CDB1, C - CDB2, $\mathrm{D}-\mathrm{CDB} 3$ e $\mathrm{E}-\mathrm{CDB} 4$. 

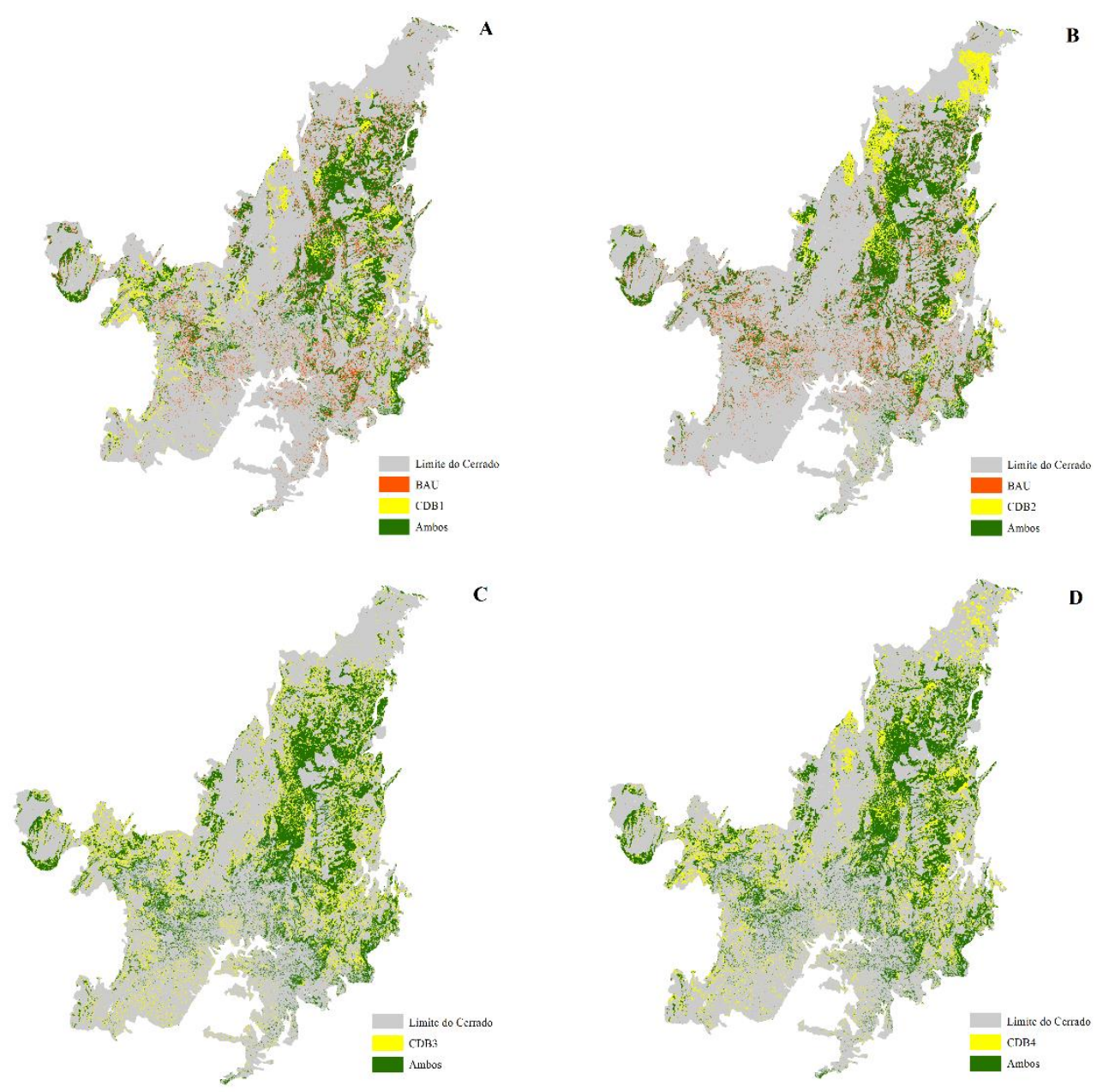

Fig. 7: Comparação entre os cenários BAU e GOV quanto à sua área remanescente de Cerrado, em 2050: A - BAU e CDB1; B - BAU e CBD2; C - BAU e CDB3 e; D - BAU e CDB4.

A estatística KIA indica uma alta congruência entre os cenários GOV. e BAU. Porém os valores de KIA são menores entre os cenários que incluem a redução na taxa de desmatamento (CDB 3 e 4), do que nos cenários onde há somente a reserva de áreas (CDB 1 e 2) (Fig. 6-7 e Tab. 3).

Tab. 3: Relação entre os cenários de acordo com a vegetação remanescente de Cerrado, calculada pelo índice Kappa (KIA)

\begin{tabular}{|lllll|}
\hline Cenários & $\mathbf{2 0 2 0}$ & $\mathbf{2 0 3 0}$ & $\mathbf{2 0 4 0}$ & $\mathbf{2 0 5 0}$ \\
\hline BAU versus CDB1 & 0.9735 & 0.9286 & 0.8701 & 0.8028 \\
BAU versus CDB2 & 0.9473 & 0.9027 & 0.8583 & 0.8030 \\
BAU versus CDB3 & 0.9111 & 0.8289 & 0.7517 & 0.6797 \\
BAU versus CDB4 & 0.9111 & 0.8288 & 0.7517 & 0.6796 \\
\hline
\end{tabular}




\subsection{A fragmentação do Cerrado}

O resultado da PCA mostrou que, independentemente do cenário, nove métricas explicam 93,04\% da variância dos dados (Fig. S4, Tab. S4).
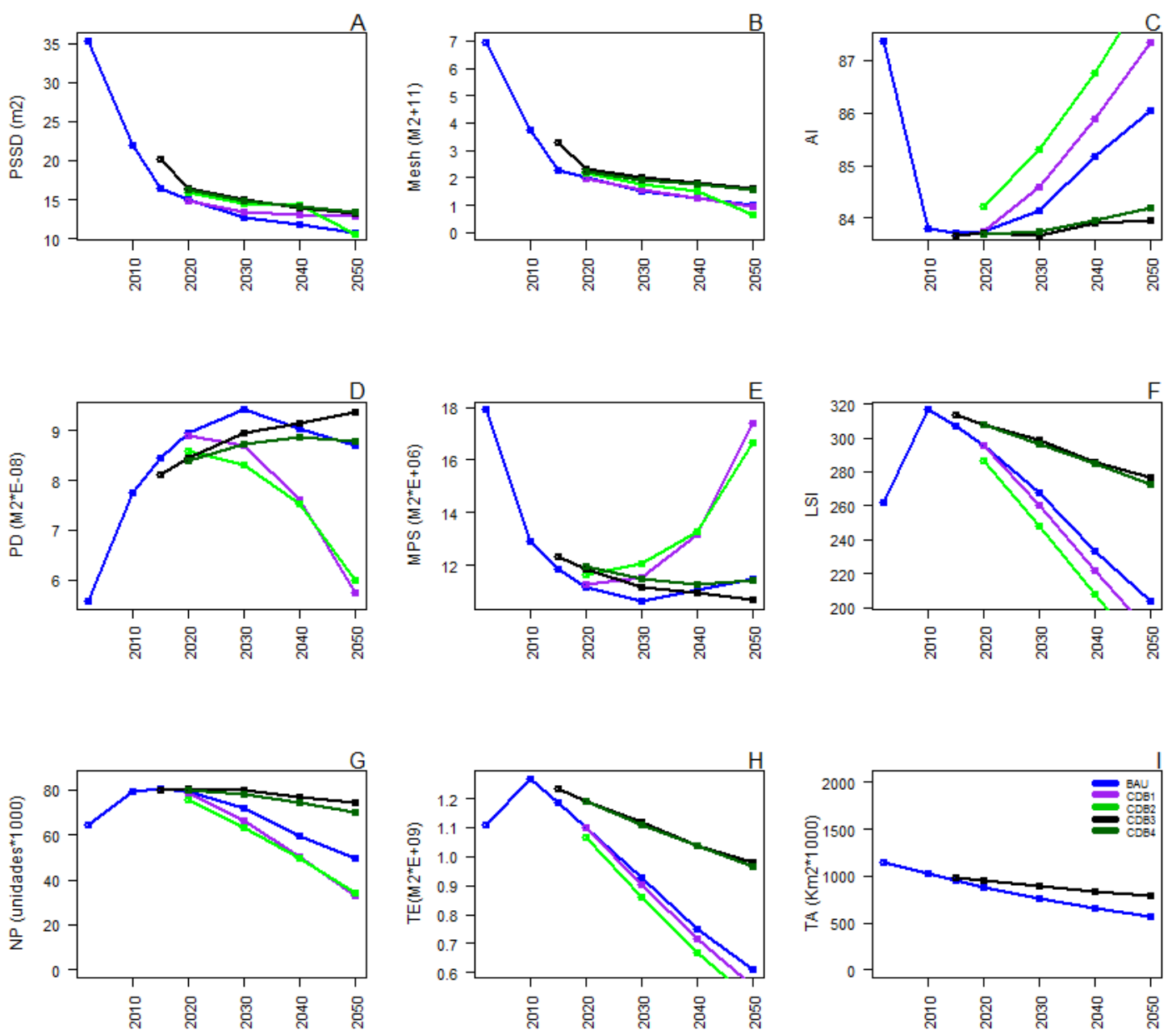

Fig. 8: Tendências dos cenários propostos em relação às métricas de fragmentos selecionadas pelo primeiro componente da PCA, de A-E (variação média: 55,29\%): desvio-padrão do tamanho dos fragmentos (PSSD); tamanho médio dos fragmentos (MPS), tamanho efetivo da malha (Mesh); índice de agregação (AI) e densidade de fragmentos (PD) e, pelo componente 2, de F-I (variação média: 37,8\%): área total (TA); número de fragmentos (NP); borda total (TE); índice de forma da paisagem (LSI). 
A análise da fragmentação (NP) (Fig. 8G) indica um aumento do número de fragmentos e, posteriormente uma queda, relacionada à quantidade de remanescentes perdidos. Os cenários que propõem a redução na taxa de desmatamento $(\mathrm{CDB} 3, \mathrm{CDB} 4)$ indicam que há uma diminuição na perda de fragmentos, enquanto que nos cenários CDB1 e CDB2, que apenas imobilizam áreas para a conservação, essa queda é mais pronunciada quando comparada ao cenário BAU. A diminuição das bordas totais (TE) (Fig. 8H) e o índice de forma da paisagem (LSI) (Fig. 8F) dos cenários seguem o mesmo comportamento de NP, pois têm uma forte correlação média com esta métrica (média da rho=0,9 e rho=0,98942, respectivamente) (Fig. S5). Nas projeções dos cenários para o período de 2010-2050, também foi observada uma maior uniformização do tamanho dos fragmentos e a diminuição de seu tamanho médio, evidenciadas neste estudo pelas métricas: desvio-padrão do tamanho dos fragmentos (PSSD) (Fig. 8A) e tamanho médio dos fragmentos (MPS), já CDB1 e CDB2 têm um aumento de MPS (Fig. 8E). As duas métricas têm alta correlação nos cenários propostos (média da rho=0,82) (Fig. S5). O índice o tamanho efetivo da malha (Mesh) (Fig. 8B) e o índice de agregação (AI) (Fig. 8C), que expressam a complexidade da paisagem indicam que, as paisagens tendem a se tornar mais desconectadas como um todo e mais agregadas em algumas regiões, sobretudo para BAU, CDB1 e CDB2. A densidade de fragmentos (PD) (Fig. 8D) mostram que em 2050, haveria um incremento de cerca de 50\% no CDB4 em relação às menores densidades (CBD1 e CDB2). Há uma correlação negativa entre o índice de agregação (AI) e densidade de fragmentos (PD) (média da rho=-0,97) (Fig. S5). LSI descreve paisagens menos desagregadas para CDB3 e CDB4 que outros cenários, provavelmente pela manutenção dos fragmentos menores.

O cenário CDB 3 será aquele que em 2050, terá o maior número de fragmentos com até 50 $\mathrm{Km}^{2}$. CDB 1 e 2 concentram as maiores áreas de fragmentos entre 1.000 e $10.000 \mathrm{Km}^{2}$ e CDB3 e CDB4 são aqueles que terão fragmentos acima de $10.000 \mathrm{Km}^{2}$ (Fig. 9 e Tab. S5). Não foram detectadas diferenças significativas entre os cenários $(F=1,0545 ; \mathrm{df}=4 ; \mathrm{p}>0,05)$, somente entre as classes de fragmentos $(\mathrm{F}=34,5998 ; \mathrm{df}=5 ; \mathrm{p}>0,001)$ (quando consideramos o número de fragmentos por classe). 


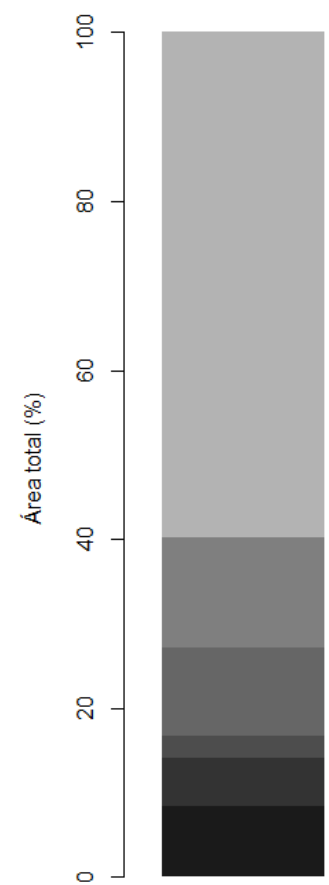

BAU

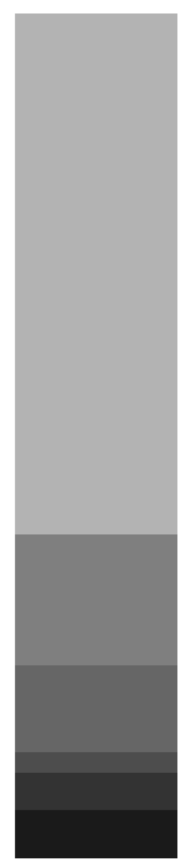

CDB 1

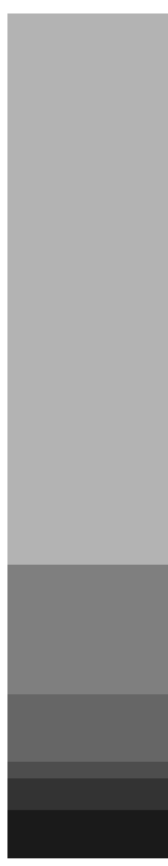

CDB 2

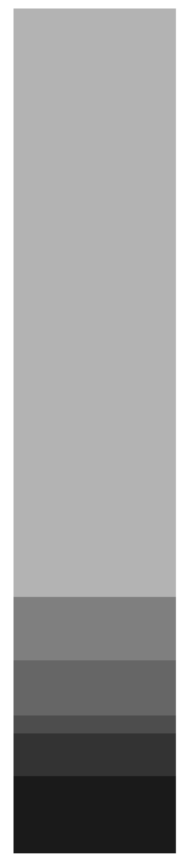

CDB 3

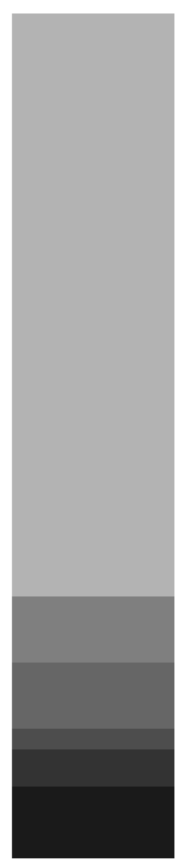

CDB 4

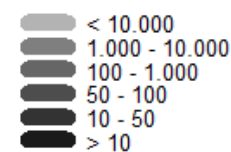

Fig 9: Distribuição de fragmentos por classes de tamanho $\left(\mathrm{Km}^{2}\right)$, considerando a área remanescente total no período de 2010-2050 e cada cenário proposto. Classes de fragmentos: $<10 \mathrm{Km}^{2}$; entre 10 e $50 \mathrm{Km}^{2}$; entre 50 e $100 \mathrm{Km}^{2}$, entre 100 e $1.000 \mathrm{Km}^{2}$, entre 1.000 e $10.000 \mathrm{Km}^{2}$ e > $10.0000 \mathrm{Km}^{2}$.

\subsection{A representatividade do Cerrado}

As Unidades de Conservação de Proteção Integral e as Terras Indígenas conservam um total de $6,91 \%$ da vegetação remanescente do Cerrado, portanto aquém das metas estipuladas pela CDB. Quatro classes de vegetação estão devidamente protegidas por Unidades de Conservação de Proteção Integral e Terras Indígenas: duas classes de vegetação de tensão ecológica $(\mathrm{Rm}=21 \%$ e $\mathrm{Tg}=83 \%)$, uma referente à floresta estacional $(\mathrm{Cb}=98 \%)$ e uma de formação pioneira $(\mathrm{Pm}=19 \%)$. As classes de vegetação típicas do Cerrado: Savana Arborizada $(\mathrm{Sa}=6 \%)$; Savana Arbórea Densa-florestada ( $\mathrm{Sd}=9 \%)$; Savana Gramíneo-lenhosa $(\mathrm{Sg}=6 \%)$ e Savana-Parque $(\mathrm{Sp}=8 \%)$, têm percentuais de proteção bem abaixo da meta proposta (17\%) (Fig. S6).

Até 2020 , os modelos indicam que pelo menos $17 \%$ de cada tipo de vegetação estariam representados (Figs. S7 e S8), com exceção da Floresta Ombrófila Densa Montana (Dm) (8\%); da Floresta Estacional Semidecidual Montana (Fm) (13\%) e da Floresta Ombrófila Mista Montana (Mm) (16\%) (Fig. S7). A resolução temporal mostra que, a partir de 2030, com o avanço do desmatamento surgem lacunas na representatividade do Cerrado (de 30 a 70\%, dependendo do cenário) (Fig. 10, Figs. S9, S10 e S11). 


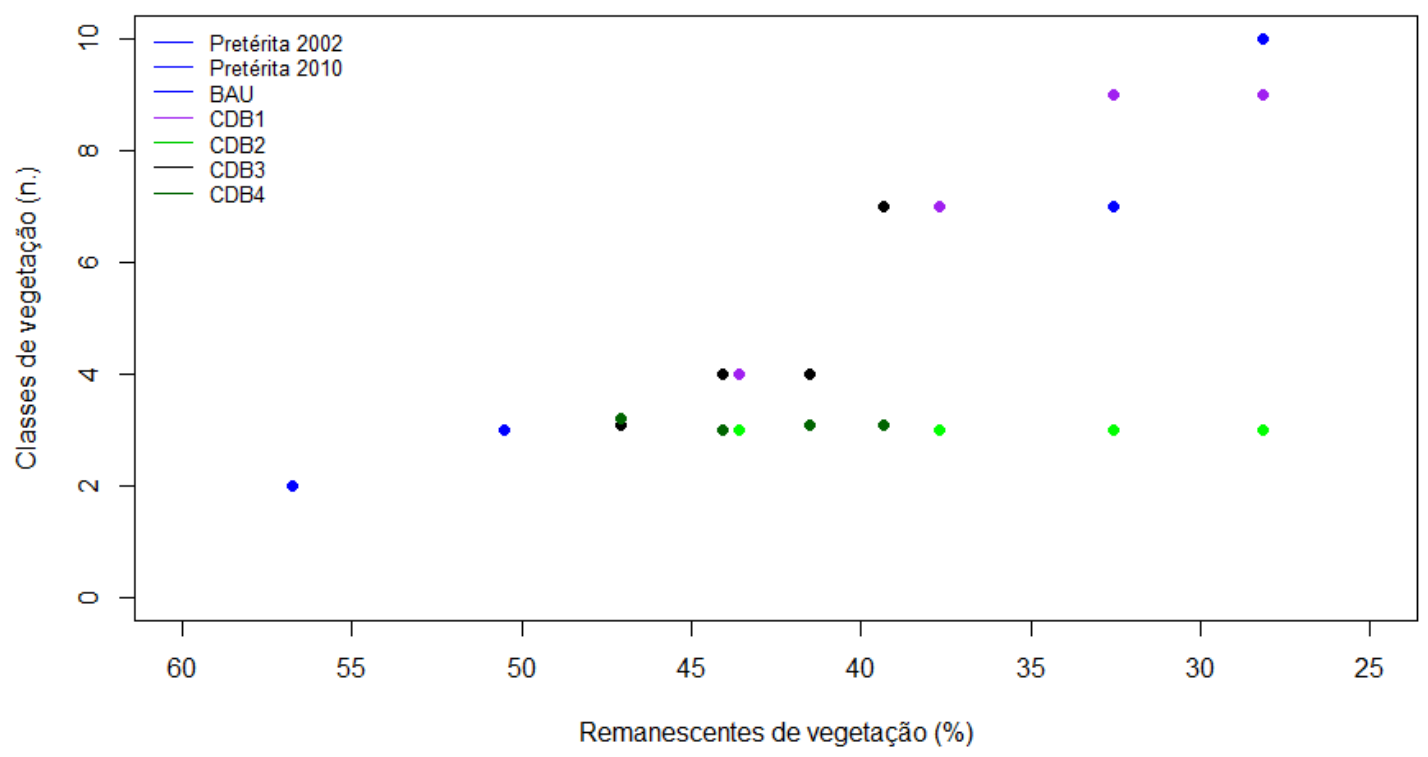

Fig. 10: Número de classes de vegetação abaixo do limiar de $17 \%$ de acordo com os remanescentes dos cenários propostos, período de 2002 a 2050.

\subsection{A vulnerabilidade do Cerrado}

Os cenários BAU e CDB3 (rho=1) e CDB2 e CDB4 (rho=0,9513) têm as maiores correlações em termos de vulnerabilidade média ao desmatamento, de acordo com as classes de vegetação (Fig.11). O aumento das ações de comando e controle, dissociadas de ações de conservação in situ não seriam suficientes para alterar os padrões de probabilidade de desmatamento do Cerrado. CDB1 e CDB2, têm a menor correlação, expressando as maiores diferenças em termos de vulnerabilidade do território (Fig. 11). 


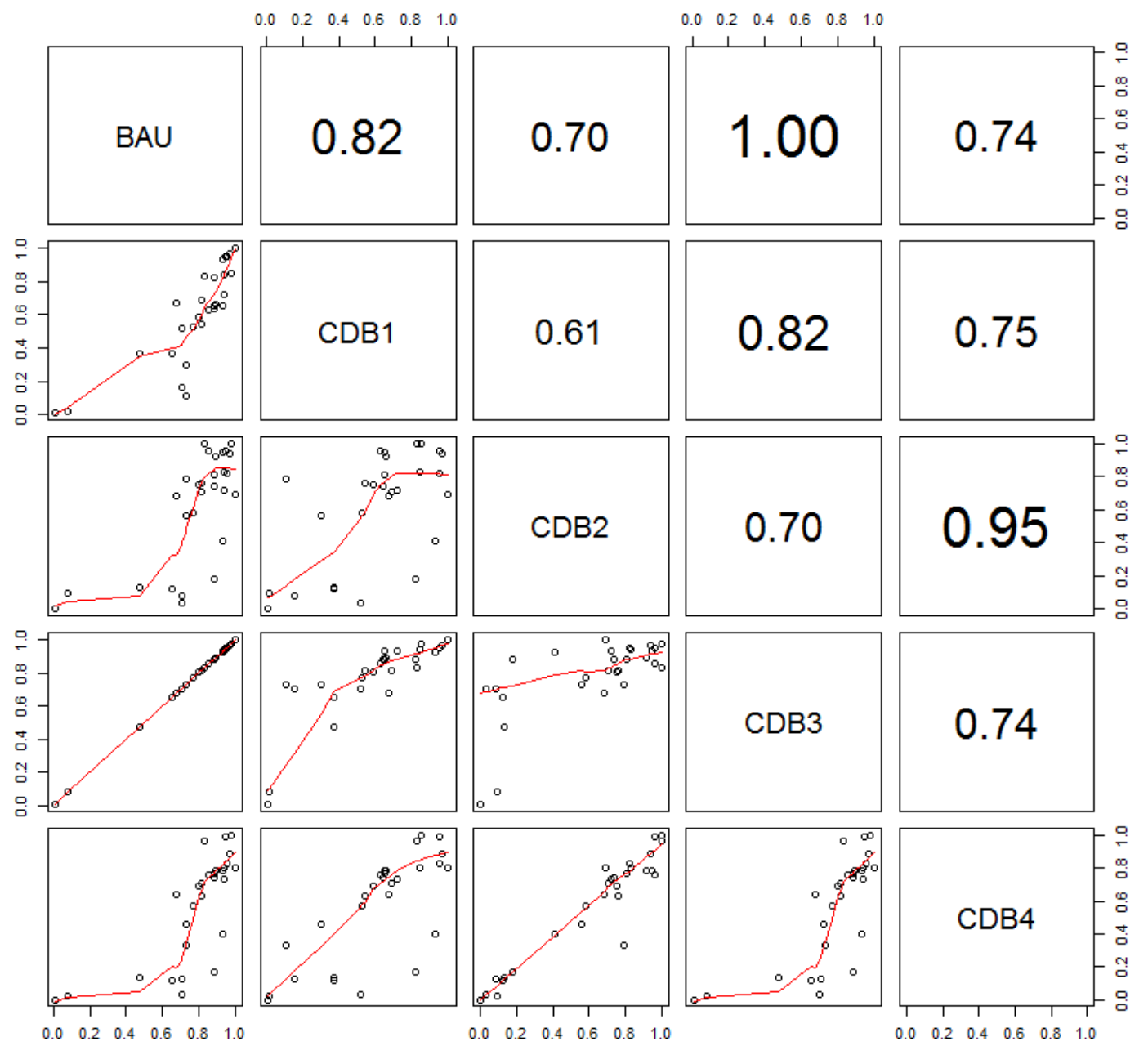

Fig. 11: Gráfico de dispersão entre as probabilidades médias de conversão das classes de vegetação, por cenário proposto e respectivos valores das análises de correlação de Spearman (em escala logarítmica); p-values $\leq 0,01$. 

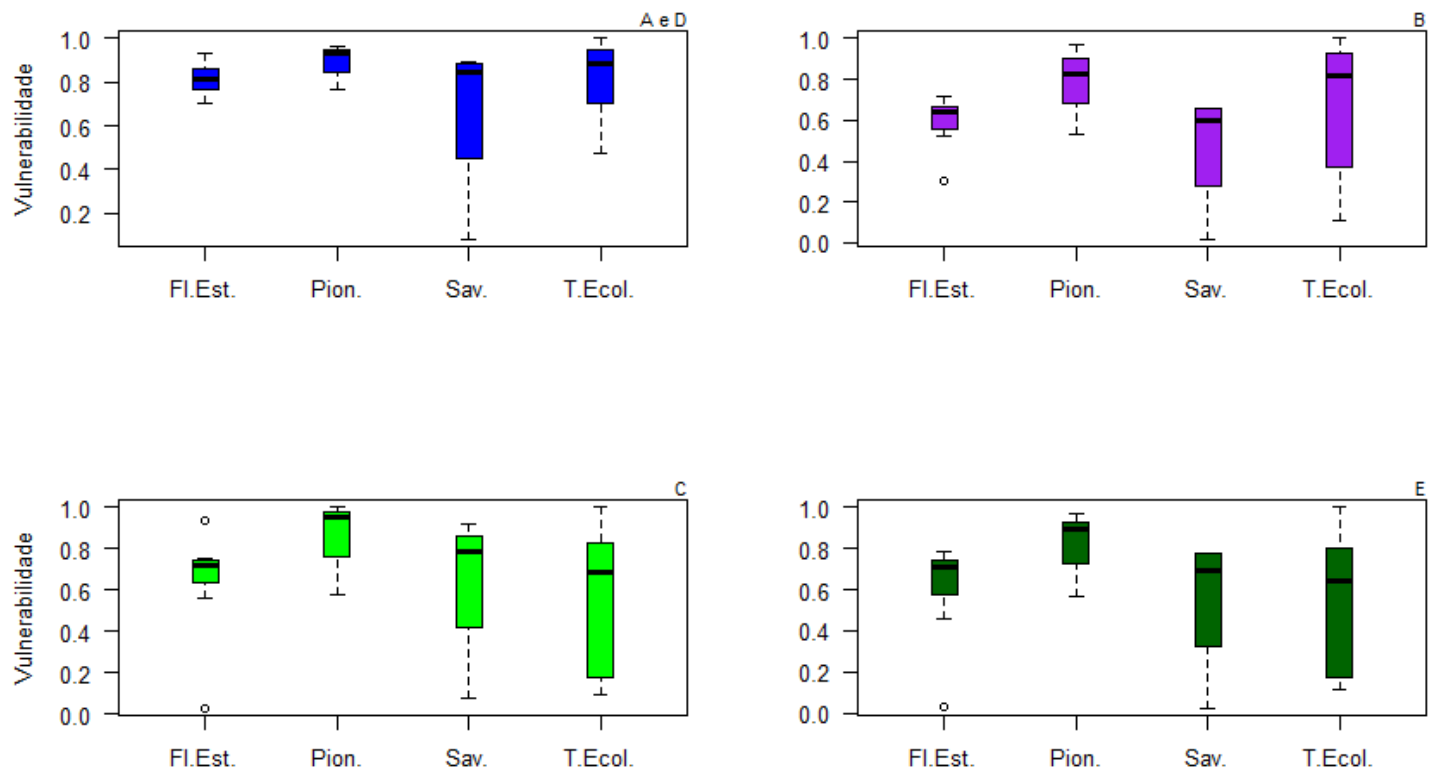

Fig. 12: Distribuição da probabilidade de conversão de áreas naturais pelas classes de vegetação agrupadas por formações vegetacionais (Florestas Estacionais - Fl.Est., Formações Pioneiras - Pion., Savanas - Sav. e Áreas de Tensão Ecológica (T.Ecol.), em cada um dos cenários: A e D - BAU e CDB3; B - CDB1; CCDB2; E-CDB4.

A distribuição da probabilidade pelas classes de vegetação se mostra uniforme entre os cenários BAU e CDB3. A pressão de desmatamento das Florestas Estacionais é reduzida nos cenários CDB1, CDB2 e CDB4 (Fig. 12 B, C e E). Já CDB1 e CDB4 diminuem a pressão sobre as Savanas (Fig. 12 B e E). 


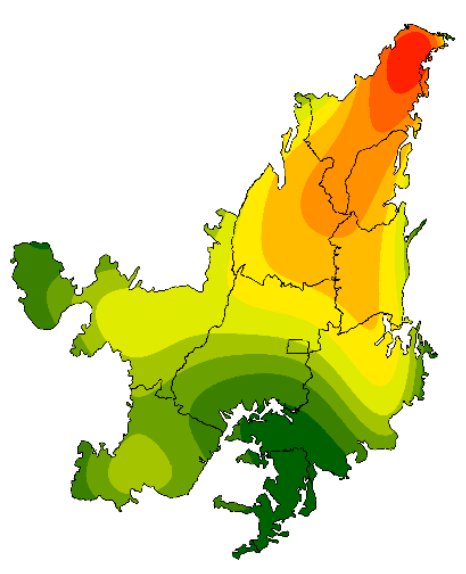

A

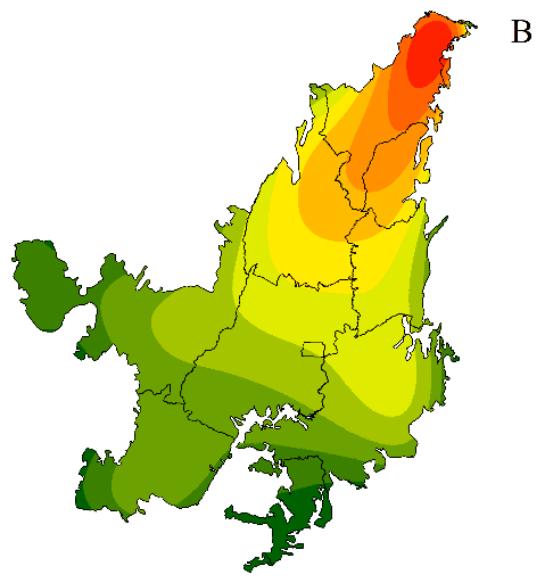

B

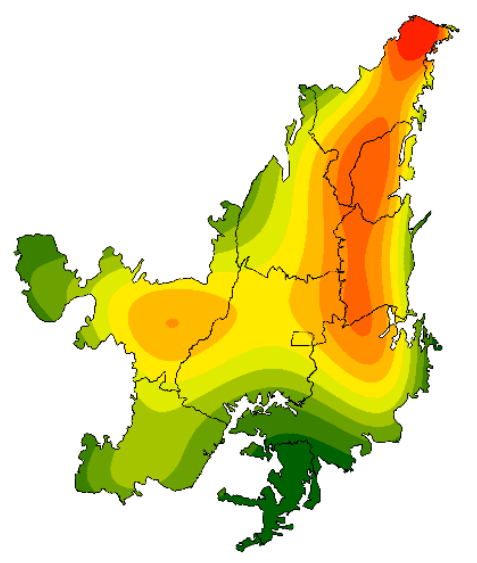

C

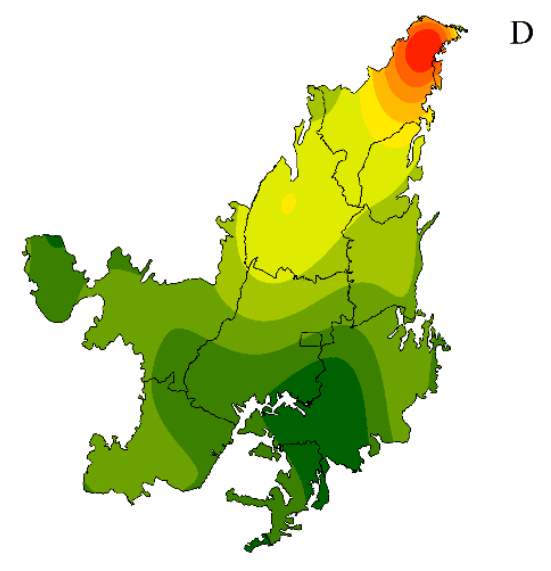

E
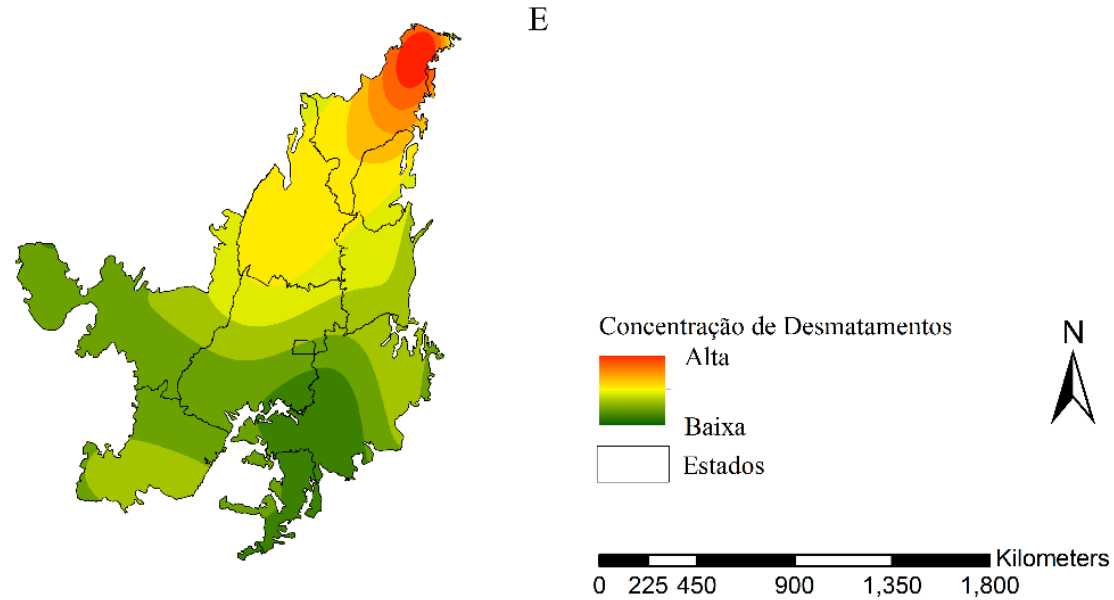

Fig. 13: Mapas mostrando tendências de desmatamentos futuros (2010 a 2050) no Cerrado, em cada cenário proposto $\left(\mathrm{A}-\mathrm{BAU}: \mathrm{R}^{2}=15,77 \% ; \mathrm{B}-\mathrm{CDB} 1 \mathrm{R}^{2}=15,42 \% ; \mathrm{C}-\mathrm{CDB} 2 \mathrm{R}^{2}=12,78 \%\right.$; $\left.\mathrm{D}-\mathrm{CDB} 3 \mathrm{R}^{2}=15,68 \% ; \mathrm{E}-\mathrm{CDB} 4 \mathrm{R}^{2}=12,69 \%\right)$. 

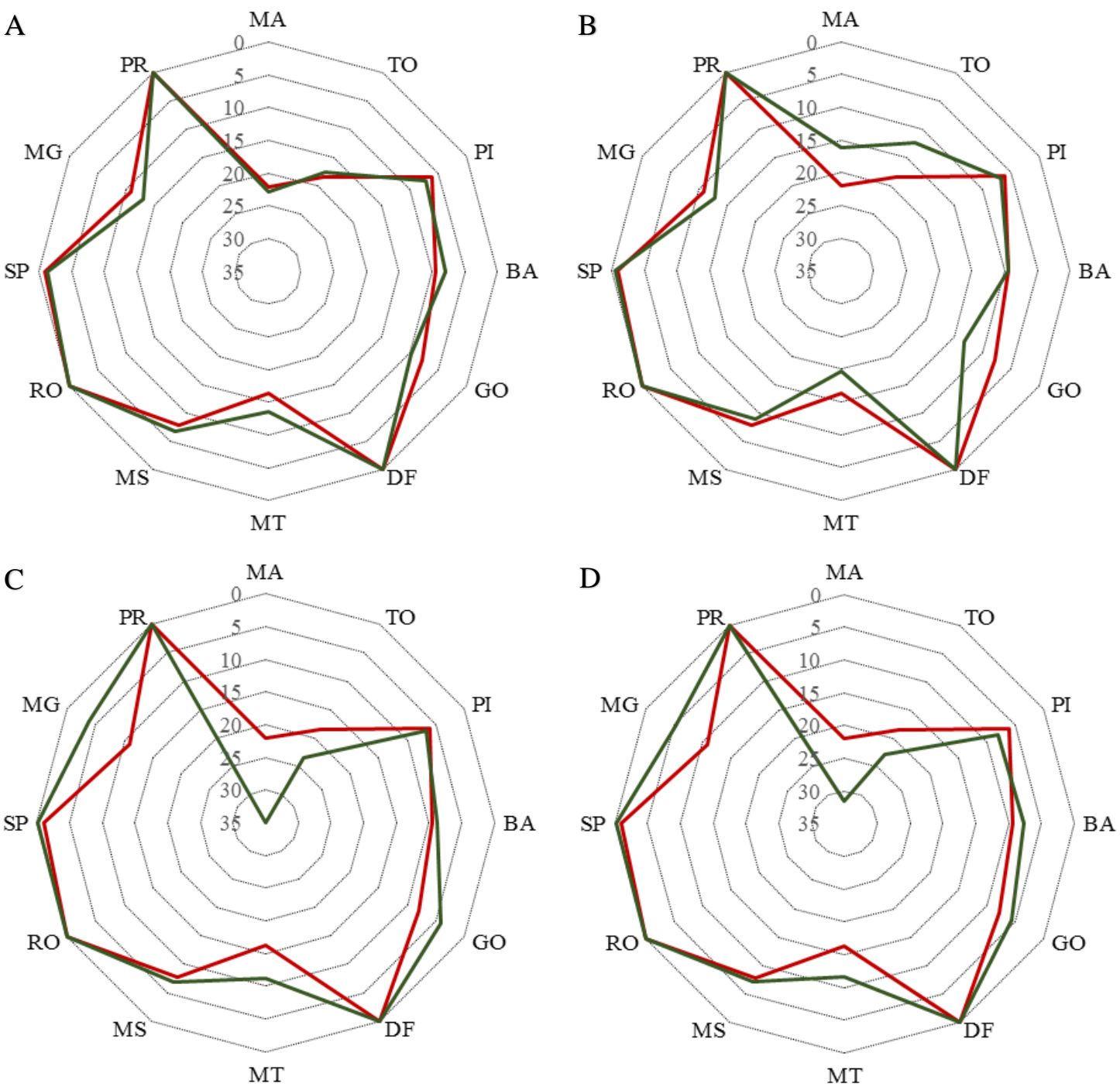

Fig. 14: Distribuição dos desmatamentos entre 2010 - 2050, por estados no Cerrado, Comparação entre o cenário BAU (linha vermelha) e cenários GOV. (linha verde) - A- CDB1; B - CDB2; C - CDB3 e D CDB4.

Quando analisados os padrões de conversão de áreas nativas do Cerrado (Fig. 13), observamos uma concentração dos desmatamentos no extremo norte do bioma, independente do cenário. CDB2 acumula taxas maiores no estado do Mato Grosso, que em outros cenários (Fig.14, Fig. S13). Nos cenários com redução das taxas de desmatamento observa-se uma diminuição da concentração de desmatamentos ao sul do bioma (Figs 13, D e E e Figs 14, C e D, Fig.S13).

\section{DISCUSSÃO}

\subsection{Os padrões de desmatamento, suas forçantes e os modelos de LUCC}

Há uma taxa líquida de desmatamento $\left(0,53 \%\right.$ ano $\left.^{-1}\right)$ de nossos cenários mais otimistas e estimamos uma perda adicional de 11,5\% na área total do Cerrado, em 2050. Nossos modelos apontam que dependendo da ação de conservação implementada, as taxas e os padrões espaciais 
de desmatamento variam entre as porções do Cerrado (estados e sub-bacias). Brannstrom et al. (2008) comparando regiões do Cerrado com diferentes históricos de ocupação, encontraram taxas líquidas de desmatamento bastante discrepantes entre si $\left(2,6 \%\right.$ ano $^{-1}$ no Oeste da Bahia e de 1,3\% ano $^{-1}$ no Mato Grosso). Avaliando os padrões de desmatamentos futuros encontramos maior vulnerabilidade nas porções centro-oeste (BAU, CDB2) e extremo norte do bioma (para todos os cenários). Contudo, ressaltamos que, no extremo norte do bioma há um viés de classificação no mapeamento utilizado (vegetação remanescente transição Caatinga/Cerrado classificada como desmatamento), assim é oportuno observar que essa tendência pode ter sido acentuada em nossas análises. Porém, outros autores (Ferreira et al., 2012 e Salmona, 2013) também observaram um direcionamento das tendências de desmatamento para o norte do bioma. A proposta de conservação de grandes blocos de remanescentes do Cerrado pelos cenários aliada às poucas opções espaciais para a ocorrência de desmatamentos, explica a grande coincidência entre as áreas conservadas em todos os cenários (KIA $\geq 0,6796)$. A conversão de áreas nativas no Cerrado ocorre, principalmente, a partir dos pequenos fragmentos (Salmona, 2013). Assim as maiores diferenças são encontradas entre BAU e os cenários CDB3 e CDB4, que preveem uma queda de $50 \%$ nas taxas de desmatamento.

O histórico de ocupação, o tamanho das propriedades, a predominância das atividades econômicas e as características físicas das áreas alteram os padrões de desmatamento (Brannstrom et al., 2008), além das forçantes econômicas, sociais e políticas. No Cerrado, a baixa declividade e regime de chuvas favoreceram a consolidação da agricultura mecanizada (Ferreira et al., 2012). Contudo, fatores antrópicos, como a infraestrutura de estradas e a proximidade com áreas desmatadas, tiveram maior associação aos desmatamentos entre $2002 \mathrm{e}$ 2010 no Cerrado ( $\phi:$ 0,9624 e $\phi:$ 0,9624, respectivamente) do que as características físicas (Fig. S1). Como há pouco acúmulo de conhecimento sobre as forçantes socioeconômicas no Cerrado (Jepson et al., 2010), podemos fazer uma analogia com o modelo de conversão do uso do solo na Amazônia, já que muitas tendências são similares entre eles (Barreto et al, 2013). Observa-se, que em alguns períodos, as taxas de desmatamento estão correlacionadas ao preço das commodities de soja e de gado (Brannstrom et al., 2011; Barreto et al., 2013; Arima et al., 2014). Além dos mercados globais, políticas de controle do desmatamento e acordos setoriais (PPCDAM e moratória da soja, respectivamente) podem ter afetado essas taxas (Rosa et al., 2014; Arima et al., 2014, Gibbs et al., 2015). De Souza et al., 2013, encontraram uma fraca correlação entre os embargos ambientais (restrição financeira aos proprietários rurais infratores) (Brasil, 2008) e a diminuição das taxas de desmatamento de pequenas áreas na Amazônia. Verburg et al., 2014 ao simular cenários, para a Amazônia, considerando o preço de commodities (de gado e da soja), as taxas de migração e a governança ambiental, observaram incremento de $41-57 \%$ nas taxas de desmatamento. 
Dados de monitoramento podem ser discrepantes entre si, quanto à sua resolução espacial (como por exemplo aqueles utilizados por Klink \& Machado et al., 2005; Ferreira et al., 2012) e em relação à metodologia empregada nos mapeamentos. Os estudos de Beuchle et al. (2015) indicaram taxas de desmatamento superiores (11.812 $\left.\mathrm{Km}^{2} / \mathrm{ano}\right)$ e menores quantitativos de remanescentes de Cerrado (47\%), em 2010, que aqueles utilizados em nossas predições (50,8\% e $6.469 \mathrm{Km}^{2} / \mathrm{ano}$ ). Ou, taxas de desmatamento inferiores como àquelas do Sistema de Detecção de Desmatamento no Cerrado - SIAD (Ferreira et al., 2007). Mas, além dos dados de entrada (Brannstrom et al., 2008), os modelos podem ser afetados pela utilização de diferentes algorítmos (Pérez-Vega et al., 2012; Ganem et al., 2013; Mas et al., 2014), diferentes resoluções espaciais e pelas variáveis empregadas nos modelos, produzindo resultados diversos (tais como os de Ferreira et al., 2012).

\subsection{A fragmentação do Cerrado}

Quando analisamos a tendência à perda de fragmentos, observamos uma trajetória do tipo parábola invertida, tal como descreve Fahrig (2003). Esta tendência é mais acentuada nos cenários BAU e CDB1 e CDB2. Essa diminuição do número de fragmentos pode estar relacionada à perda de fragmentos completos (Machado et al., 2012; Stan et al., 2015), pois há uma correlação moderada (média de rho= 0.64) com a área total remanescente (Fig. S5). Essa análise também carrega o efeito da resolução espacial utilizada no estudo, pois, de fato, fragmentos menores que $0,25 \mathrm{Km}^{2}$ não foram analisados. Stan et al. (2015) observaram que o desaparecimento dos fragmentos menores ocorre principalmente pela proximidade às áreas já convertidas, por causa dos menores custos com logística e comercialização da produção agrícola. CDB1 e CDB2 têm uma menor diminuição do tamanho médio dos fragmentos e, pela perda dos fragmentos menores, têm uma tendência a homogeneização de seus tamanhos ao longo do tempo (Stan et al., 2015), promovendo também uma maior agregação da paisagem. Isso ocorre quando as iniciativas de conservação estão dissociadas das quedas das taxas do desmatamento e tendem a conservar blocos contíguos de remanescentes. Há uma forte indicação que esses cenários contribuirão para paisagens ainda mais desconectadas, principalmente na porção sul do bioma. De fato, propostas de áreas protegidas desconectadas de outras ações de comando e controle, não serão suficientes para conter a contínua fragmentação do Cerrado (Ganem et al., 2013).

Os cenários CDB3 e CDB4, que incluem a queda na taxa de desmatamento, conservam o maior percentual de áreas acima de $10.000 \mathrm{Km}^{2}$, corroborando Stan et al. (2015), que simulando cenário de governança ambiental (no estado de Minas Gerais) observaram que a diminuição das taxas de desmatamento implicaram na conservação dos maiores fragmentos.

Ainda não é possivel compreender a totalidade do impacto dos processos de fragmentação nas espécies e nos ecossistemas, mas pode-se inferir que esse decréscimo na conectividade 
estrutural pode levar a um rápido aumento na taxa de extinção das espécies (Metzger \& Décamps, 1997; Haddad et al., 2015). O declínio pode se tornar mais evidente logo após o evento de fragmentação, ou depois de muitos anos e esse limiar de perda por fragmentação pode alterar a riqueza de espécies entre 20 a 75\%, dependendo da espécie (Haddad et al., 2015). Essas taxas oscilam de acordo com características biológicas das espécies (Metzger \& Décamps, 1997). Para espécies com baixa capacidade de dispersão e alto requerimento de área, pode variar entre $40 \mathrm{a}$ 60\% (McLellan et al., 1986 apud Metzger \& Décamps, 1997) ou entre 25\% a 50\% para espécies com altas taxas de fecundidade e de sobrevivência (Lande's, 1987). A partir de $20 \%$ de fragmentação de hábitats, problemas com a persistência das espécies começam a se tornar evidentes (Fahrig, 2001). Esses limiares ambientais também podem alterar qualitativamente os processos ambientais (Turner, 1989).

\subsection{A conservação do Cerrado e as metas da CDB}

Ambientes com alta representatividade e muito fragmentados são causas perdidas? As análises dos padrões de fragmentação dos cenários propostos nos permitem demonstrar a importância de aliar a conservação dos grandes blocos de remanescentes, garantindo a persistência dos processos ecológicos, a manutenção dos pequenos fragmentos que guardam parte da representatividade do bioma, os quais estão expressos pelos cenários CDB3 e CDB4. Estudos sobre a fragmentação de hábitats podem auxiliar na condução das políticas públicas (Rosa et al., 2014b). Os hábitats na região centro-sul do bioma concentram as maiores riquezas de espécies de aves, mamíferos e anuros (Diniz-Filho et al., 2009), contudo são áreas com alta densidade populacional (Barreto et al., 2008) e já muito fragmentadas. Por outro lado, os grandes blocos de remanescentes de vegetação, que podem maximizar a persistência das espécies e garantir muitos dos processos ecossistêmicos, reduzindo a conversão de hábitats naturais (Carranza et al., 2014). Brannstrom et al. (2008) analisando duas frentes de expansão da fronteira agrícola no Cerrado com características físicas e histórico de ocupação diversos (no Oeste da Bahia e no leste do Mato Grosso), sugerem que as políticas de conservação devem ter abordagens adequadas às suas diferenças regionais. Estes autores indicaram que a criação/expansão de áreas protegidas no Cerrado é viável, sobretudo na porção norte do bioma, que guarda grande parte de sua área remanescente (Sano et al., 2010), mas que também coincide com áreas de fronteira agrícola. Enquanto que em regiões com alta fragmentação (como é o caso do leste do Mato Grosso) políticas de comando e controle podem ser mais factíveis.

Os corredores ecológicos podem aumentar a oferta de hábitats, garantir a dispersão e migração e o fluxo gênico e consequentemente a funcionalidade dos fragmentos. O Plano Estratégico Nacional de Áreas Protegidas - PNAP (Brasil, 2006b), buscam a reconexão entre as Áreas Protegidas e, Reservas Legais (RLs) e Áreas de Preservação Permanentes (APPs) de 
propriedades particulares. Ganem et al., 2013 avaliam que as organizações não-governamentais têm tido alguns sucessos na implementação dessas políticas mas que, as ações de governo ainda são tímidas e estão muito aquém das necessidades urgentes do Cerrado. Instrumentos como o Cadastramento Ambiental Rural (CAR), que identificam as áreas rurais e suas respectivas RLs e APPs, podem ser um passo importante para restaurar parte da conectividade do bioma. As metas nacionais consideram que essas áreas legalmente protegidas e as TIs, entram no cômputo juntamente com as unidades de conservação (Brasil, 2013b).

Barreto et al., 2013, observaram que no período de 1996 a 2006, houve a expansão de áreas agrícolas e aumento da produção nos estados do Maranhão e Tocantins. O encontro desses dois estados, juntamente com o Piaú e Bahia, compõe uma região denominada como Matopiba, área de expansão da fronteira agrícola no Cerrado. Os arranjos produtivos, históricos de ocupação e organizações político-sociais ali instalados dificultam a gestão ambiental destes territórios (Brannstrom et al., 2005). O marco regulatório do "Plano de Desenvolvimento Agropecuário de Matopiba", não prevê a gestão da biodiversidade em seu escopo (Brasil, 2015b). O que corrobora os estudos que antecipam que Matopiba deverá concentrar os maiores índices de desmatamento no futuro (Ferreira et al., 2012, Salmona, 2013). Nessa região existem ainda $20.000 \mathrm{Km}^{2}$ de áreas produtivas suscetíveis a invasão legalizada pela soja, e estas atividades substituídas (em geral pastagens) deverão impulsionar o desmatamento de novas áreas, no Cerrado ou na Amazônia (Gibbs et al., 2015. Por outro lado, Matopiba é onde se localizam as maiores unidades de conservação de proteção integral do Cerrado, como o Parque Nacional Nascentes do Parnaíba $\left(7.400 \mathrm{Km}^{2}\right)$ e a Estação Ecológica da Serra Geral do Tocantins $\left(7.160 \mathrm{Km}^{2}\right)$. Assim, não somente as áreas nativas remanescentes mas também as unidades de conservação estão sob forte pressão antrópica, oficializada pelo supracitado Plano do Matopiba.

A Política Nacional do Meio Ambiente (Brasil, 1981) institui entre os seus instrumentos, o zoneamento ecológico-econômico e a avaliação de impactos ambientais, que buscam o desenvolvimento do território integrando os planos setoriais e promovendo o equilíbrio ecológico. Contudo, o que podemos observar é que no país há pouca cultura para o planejamento, sobretudo àqueles ligados às questões ambientais, que são vistos como um mero cumprimento das formalidades impostas pela legislação ambiental. Há pouca integração entre as políticas de biodiversidade e outros setores do governo (Prates \& Irving, 2015).

As COPs estabeleceram objetivos, metas e indicadores globais que devem ser os norteadores para o estabelecimento de metas nacionais, consideradas as particularidades de cada país-membro. Mas, como essas metas globais protegem nossa biodiversidade? Para responder a esse questionamento é preciso traduzí-las em metas quantitativas e operacionais, que podem ser mais facilmente defendidas pelos tomadores de decisão em fóruns setoriais (Pressey, et al., 2003). 
A avaliação dessas metas deve ser pautada no conhecimento sistemático sobre os alvos da conservação, que devem ser revistas periodicamente, à luz de novos conhecimentos científicos (Pressey et al., 2003, Svancara et al., 2005) e à medida que as ações para a conservação forem implementadas (Pressey \& Taffs, 2001). Alguns estudos demostram que as metas lineares propostas pela CDB podem ser inadequadas para espécies ameaçadas, endêmicas e/ou com distribuição pequena (De Novaes e Silva et al., 2014). Neste estudo, encontramos alguns objetos de conservação que exigiriam medidas de restauração, pois em 2010, já apresentavam um quantitativo de área abaixo da meta proposta, e em períodos futuros e, alguns cenários mostram uma tendência ao aumento dessas lacunas de representatividade (de até 30\% em CDB2 e CDB4; e de até 90\% em CDB1) (Tab. S6). O estudo de LUCC, pode revelar componentes da biodiversidade vulneráveis que necessitarão de proteção adicional, de acordo com prognósticos futuros. Adequar as metas assumidas no contexto da $\mathrm{CDB}$ às necessidades da biodiversidade (Svancara et al., 2005) pode representar uma oportunidade para a o planejamento da conservação no país (Machado et al., 2012b).

Quanto às metas de conservação, a missão que o governo do Brasil se impôs é ambiciosa (Weigand Jr et al., 2011). Nossos cenários implicam na proteção de 12 a 27\% da área total do Cerrado (cenário CDB1 e CDB4, respectivamente), o que significaria a criação e/ou gestão de mais de $238.000 \mathrm{Km}^{2}$ de áreas legalmente protegidas até 2020. Entre 2010 e 2014, no Cerrado, foram decretadas apenas 3 UCs de proteção integral $\left(79,55 \mathrm{Km}^{2}\right)$, e uma de uso sustentável no ecótono Mata Atlântica/Cerrado, com $381,77 \mathrm{Km}^{2}$.

\section{CONCLUSÃO}

Por mais árdua que seja a busca de resposta de "Quanto hábitaté necessário?” (Fahrig, 2001) para um país com dimensões continentais e com pouca experiência em planejar a conservação, é preciso enfrentar o desafio. O caminho começa pela definição dos objetivos da conservação, seguido pelo desenvolvimento de indicadores de biodiversidade para o monitoramento ambiental, da revisão das metas e da elaboração de prognósticos futuros. Os modelos espacialmente explícitos podem conduzir experimentos que nos ajudarão a entender os processos chaves para o desenvolvimento e conservação do território (Veldkamp \& Lambin, 2001). No presente estudo, buscamos, primeiro, espacializar as metas brasileiras, propondo cenários exploratórios, avaliando qual a efetividade de proteção oferecida por elas, nos tempos futuros, utilizando para isso, os modelos espacialmente explícitos de uso e ocupação do solo (LUCC). Nossos resultados mostram que os cenários oferecem diferentes graus de proteção, alterando a vulnerabilidade do território, a estrutura da paisagem e a representatividade dos alvos de proteção selecionados. O cenário que combina ações de comando e controle com a governança ambiental demonstra a necessidade da utilização de diferentes estratégias para a conservação do Cerrado. As análises em macroescalas 
oferecem padrões de desenvolvimento do território e podem subsidiar um sistema hierárquico de tomada de decisões (Diniz-Filho et al. 2009). Os modelos regionais representam apenas uma parte da complexidade dos processos envolvidos no estudo (Veldkamp \& Lambin, 2001) e mostram padrões espaciais pertinente quanto à sua escala (Diniz-Filho et al., 2009). O Brasil internalizou em seus instrumentos normativos, em suas estruturas institucionais e democráticas muitas das recomendações propostas pela CDB (Prates \& Irving, 2015), mas ainda precisa resolver suas contradições internas alavancadas pelas disputas setoriais dos territórios. Contudo, a CDB ainda é um vislumbre das oportunidades que poderá representar para a conservação, especialmente no Cerrado brasileiro. 


\section{REFERÊNCIAS BIBLIOGRAFICAS}

Abreu, C.G.; Coelho, C.G.C.; Ralha, G. Zabhetto, A.; Macchiavello, B., 2011. MASE: A Multi-Agent System for Environmental Simulation. Environ. Model. Softw. 1-32.

Aguiar, T.D.J.A. De, Monteiro, M.D.S.L., 2005. Modelo Agrícola e Desenvolvimento Sustentavél: A ocupação do Cerrado Piauiense. Ambient. Soc. 8, 1-17. doi:10.1590/S1414-753X2005000200009

Alho, C. J. R., Martins, E. D. S., 1995. De Grão em Grão, o Cerrado Perde Espaços: CerradoImpactos do Processo de Ocupação. Fundo Mundial para a Natureza/PROCER, Brasília, DF.

Arima, E.Y., Barreto, P., Araújo, E., Soares-Filho, B., 2014. Land Use Policy Public policies can reduce tropical deforestation: Lessons and challenges from Brazil. Land Use Policy 41, 465-473. doi:10.1016/j.landusepol.2014.06.026

Ball, I. R. \& Possingham, H. P., 2000. MARXAN (V1.8.2): Marine Reserve Design Using Spatially Explicit Annealing, a Manual, University of Queensland, Brisbane, Australia.

Ball, I. R., Possingham, H. P.; Watts, M. 2009. Marxan and relatives: Software for spatial conservation prioritization. In: Moilanen, A., Wilson, K.A.; Possingham H. P. (Eds)., 2009. Spatial conservation prioritization: Quantitative methods and computational tools. Oxford University Press: Oxford (UK), p.185-195.

Balmford, A. Bennun, L. Brink, B. ten; Cooper, D. Côté, I. M.; Crane, P. Dobson, A.; Dudley and Dutton, N.; Green R. E. ;Gregory, R. D.; Harrison, J. Kennedy, E. T.; Kremen, C.; LeaderWilliams, N.; Lovejoy, T. E.; Mace, G.; May, R.; Mayaux, P.; Morling, P. Philips, J.; Redford, K.; Ricketts, T. H.; Rodriguez, J. P.; Sanjayan, M.; Schei, P. J.; Jassrsveld, A.S.van \& Walther, B. A., 2005 The Convention on Biological Diversity's 2010 target. Himalayan Jounal of Sciences, v.3, n.5, Jan-Jun.

Barreto, B.D.S., Oliveira, G. De, Pinto, M.P., Mauricio, L., Alexandre, J., Diniz, F., Blamires, D., 2008. Riqueza de espécies de emberizídeos e conflitos de conservação no Cerrado brasileiro. Acta Sci.Biol.Sci. 67-72.

Barretto, A.G.O.P., Berndes, G., Sparovek, G., Wirsenius, S., 2013. Agricultural intensification in Brazil and its effects on land-use patterns: an analysis of the 1975-2006 period. Glob. Chang. Biol. 19, 1804-15. doi:10.1111/gcb.12174

Baudron, F.\& Giller, K. E., 2010. Agriculture and nature: Trouble and strife? Biological Conservation, 170, 232-246.

Beuchle, R., Grecchi, R.C., Shimabukuro, Y.E., Sano, E.E., Achard, F., 2015. Assessing land cover changes in the Brazilian Cerrado between 1990 and 2010 using a remote sensing sampling approach. Appl. Geogr. 58, 116-127.

Brandão, A.S.P., Rezende, G.C. De, Marques, R.W.D.C., 2006. Crescimento agrícola no período 1999/2004: a explosão da soja e da pecuária bovina e seu impacto sobre o meio ambiente. Econ. Apl. 10, 249-266.

Brannstrom, C., 2005. Environmental policy reform on north-eastern Brazil's agricultural frontier. Geoforum 36, 257-271. doi:10.1016/j.geoforum.2004.06.002 
Brannstrom, C., Jepson, W., Filippi, A.M., Redo, D., Xu, Z., Ganesh, S., 2008. Land change in the Brazilian Savanna (Cerrado), 1986-2002: Comparative analysis and implications for land-use policy. Land Use Policy 25, 579-595. doi:10.1016/j.landusepol.2007.11.008

Brannstrom, C., Rausch, L., Brown, J.C., de Andrade, R.M.T., Miccolis, A., 2011. Compliance and market exclusion in Brazilian agriculture: Analysis and implications for "soft" governance. Land Use Policy 29, 357-366. doi:10.1016/j.landusepol.2011.07.006

Brasil, 1981. Lei n 6.938, de 31 de Agosto de 1981.

Brasil, 1992. Manuais Técnicos em Geociências n.1. Manual Técnico da Vegetação Brasileira n.1. Rio de Janeiro, IBGE.

Brasil, 1993. Mapa de Vegetação do Brasil. Fundação Instituto Brasileiro de Geografia e Estatística, Ministério da Agricultura.

Brasil, 2000. A Convenção sobre Diversidade Biológica (CDB). Série Biodiversidade: Ministério do Meio Ambiente (MMA), Brasília: MMA/SBF, 404p..

Brasil, 2000b. Lei nº 9.985, de 18 de Julho de 2000.

Brasil, 2002. Decreto $n^{0 .} 4.339$, de 22 de Agosto de 2002.

Brasil, 2004. Mapa de Biomas do Brasil. Escala 1.5.000.000. Instituto Brasileiro de Geografia e Estatística - IBGE, Rio de Janeiro.

Brasil, 2004b. Portarias MMA nº 126, 27 de Maio de 2004.

Brasil, 2006. Resolução CONABIO n 03, de 21 de dezembro de 2006.

Brasil, 2006b. Decreto No 5.758, de 13 de Abril de 2006.

Brasil, 2007. Portaria MMA nº 9, de 23 de janeiro de 2007.

Brasil, 2007b. Biodiversidade brasileira: avaliação e identificação de áreas e ações prioritárias para conservação, utilização sustentável e repartição de benefícios da biodiversidade brasileira. Brasília: Ministério do Meio Ambiente (MMA), 31, 300p.

Brasil, 2008. Decreto n ${ }^{\circ} 6.514$ de 22 de Julho de 2008.

Brasil. Ministério da Ciência e Tecnologia. Coordenação-Geral de Mudanças Globais do Clima. Segunda Comunicação Nacional do Brasil à Convenção-Quadro das Nações Unidas sobre Mudança do Clima. Brasília: Ministério da Ciência e Tecnologia., 2010. 2v. il.col.map.; $30 \mathrm{~cm} .+\mathrm{CD}-\mathrm{ROM}\left(4 \frac{3}{4} \mathrm{in}\right.$.).

Brasil, 2010b. Diretoria do Programa Nacional de Conservação da Biodiversidade - DCBio, Quarto Relatório Nacional para a Convenção sobre Diversidade Biológica. Brasília: Ministério do Meio Ambiente (MMA).

Brasil, 2010c. Decreto nº 7.390, 9 de Dez. de 2010.

Brasil, 2011. Monitoramento do bioma Cerrado 2002 a 2008. Monitoramento do desmatamento nos biomas brasileiros por satélite: acordo de cooperação técnica MMA/IBAMA. Relatório 
técnico, Instituto Brasileiro do Meio Ambiente e dos Recursos Naturais Renováveis. http://www.mma.gov.br/portalbio.

Brasil, 2012. Lei ${ }^{\circ}$ 12.651, de 25 de Maio de 2012.

Brasil, 2013. Base digital de dados geoespacializados das áreas protegidas do Brasil, compilados pela Diretoria de Qualidade Ambiental do Ibama, com dados do Cadastro Nacional de Unidades de Conservação e órgãos estaduais e municipais de meio ambiente (atualizado até 01/02/2013).

Brasil, 2013b. Base digital de dados geoespacializados das áreas prioriárias do Cerrado, dados não-publicados.

Brasil, 2013c, Resolução CONABIO nº.6, de 3 de Setembro de 2013.

Brasil, 2014. PNIA 2012: Painel Nacional de Indicadores Ambientais.MMA: Brasília, DF.97p.

Brasil, 2015, disponível em http://www.mma.gov.br/florestas/controle-e-prevencao-dodesmatamento

Brasil, 2015. Decreto ${ }^{\circ}$ 8.447, de 6 de Maio de 2015.

Brooks, T.M., Mittermeier, R. a., da Fonseca, G. a. B., Gerlach, J., Hoffmann, M., Lamoreux, J.F., Mittermeier, C.G., Pilgrim, J.D., Rodrigues, a S.L., 2006. Global Biodiversity Conservation Priorities. Science (80-. ). 313, 58-61. doi:10.1126/science.1127609

Bruner, a G., Gullison, R.E., Rice, R.E., da Fonseca, G. a, 2001. Effectiveness of parks in protecting tropical biodiversity. Science (80-. ). 291, 125-8. doi:10.1126/science.291.5501.125

Callicott, J.B., Rozzi, R., Delgado, L., Monticino, M., Acevedo, M., Harcombe, P., 2007. Biocomplexity and conservation of biodiversity hotspots: three case studies from the Americas. Philos. Trans. R. Soc. Lond. B. Biol. Sci. 362, 321-33. doi:10.1098/rstb.2006.1989

Carranza, T., Balmford, A., Kapos, V., Manica, A., 2014. Protected area effectiveness in reducing conversion in a rapidly vanishing ecosystem: The Brazilian Cerrado. Conserv. Lett. 7, 216223. doi:10.1111/conl.12049

Carranza, T., Manica, A., Kapos, V., Balmford, A., 2014b. Mismatches between conservation outcomes and management evaluation in protected areas: A case study in the Brazilian Cerrado. Biol. Conserv. 173, 10-16. doi:10.1016/j.biocon.2014.03.004

CBD-Convention of biological diversity, 1992. United Nations. Concluded on 5 June 1992. Rio de Janeiro, Brazil.

CDB, 2013. Acessado em 12/03/2014. https://www.cbd.int/

Clark Labs.,2009. Land change modeler. Acessado em 12/03/2014. https://clarklabs.org/category/white-papers/

Cowling, R. M., R. L. Pressey, A. T. Lombard, P. G. Desmet, and A. G. Ellis.,1999. From representation to persistence: requirements for a sustainable system of conservation areas in 
the species rich Mediterranean-climate desert of southern Africa. Biodiversity and Distributions.5, 51-71.

De Souza, R.A., De Marco, P., 2014. The use of species distribution models to predict the spatial distribution of deforestation in the western Brazilian Amazon. Ecol. Modell. 291, 250-259. doi:10.1016/j.ecolmodel.2014.07.007

Eiten, G., 1972. The Cerrado vegetation of Brazil. Bot. Rev. 38, 201-341.

De Novaes e Silva, V., Pressey, R.L., Machado, R.B., VanDerWal, J., Wiederhecker, H.C., Werneck, F.P., Colli, G.R., 2014. Formulating conservation targets for a gap analysis of endemic lizards in a biodiversity hotspot. Biol. Conserv. 180, 1-10. doi:10.1016/j.biocon.2014.09.016

Diniz-Filho, J.A.F., Bini, L.M., Oliveira, G., Barreto, B.D.S., Silva, M.M.F.P., Terrible, L.C., Rangel, T.F.L.V.B., Pinto, M.P., Sousa, N.P.R., Vieira, L.C.G., Melo, A.S., de Marco Júnior, P., Vieira, C.M., Balmires, D., Bastos, R.P., Carvalho, P., Ferreira, L.G., Telles, M.P.D.C., Rodrigues, F.M., Silva, D.M., Silva Júnior, N.J., Soares, T.N., 2009. Macroecologia, biogeografia e áreas prioritárias para conservação no cerrado. Oecologia Bras. 13, 470-497. doi:10.4257/oeco.2009.1303.05

Eastman, J.R., 2009. IDRISI 16: The Taiga Edition (Worcester, MA: Clark University).

ESRI 2015. ArcGIS Desktop: Release 10.3.1. Redlands, CA: Environmental Systems Research Institute.

Fahrig, L., 2001. How much habitat is enough? Biol. Conserv. 100, 65-74. doi:10.1016/S00063207(00)00208-1

Fahrig, L., 2003.Effects of habitat fragmentation on biodiversity. Annual Review of Ecology Evolution and Systematics, 34, 487-515.

Fairbanks, D., 2001. Species and environment representation: selecting reserves for the retention of avian diversity in KwaZulu-Natal, South Africa. Biol. Conserv. 98, 365-379. doi:10.1016/S0006-3207(00)00179-8

Faleiro, F. V., Loyola, R.D., 2013. Socioeconomic and political trade-offs in biodiversity conservation: a case study of the Cerrado Biodiversity Hotspot, Brazil. Divers. Distrib. 19, 977-987. doi:10.1111/ddi.12072

Faleiro, F. V., Machado, R.B., Loyola, R.D., 2013b. Defining spatial conservation priorities in the face of land-use and climate change. Biol. Conserv. 158, 248-257. doi:10.1016/j.biocon.2012.09.020

Ferreira, M.E.; Ferreira Jr. L.G.; Huete, A.R.; Ferreira, M.E., 2007. An Operational Deforestation Mapping System Using MODIS Data and Spatial Context Analysis. International Journal of Remote Sensing, 28, 47-62.

Ferreira, M.E., Ferreira, L.G., Miziara, F., Soares-Filho, B.S., 2012. Modeling landscape dynamics in the central Brazilian savanna biome: future scenarios and perspectives for conservation. J. Land Use Sci. 1-19. doi:10.1080/1747423X.2012.675363 
Fischer, D.T. \& Church, R.L., 2003. Clustering and compactness in reserve site selection: An extension of the Biodiversity management área seletion model. Forest Science, 49, 555-564.

Ganem, R.S., Drummond, J. A, Franco, J.L. A, 2013. Conservation polices and control of habitat fragmentation in the brazilian Cerrado Biome. Ambient. Soc. 16, 99-118. doi:10.1590/S1414-753X2013000300007

Gibbs, B.H.K., Rausch, L., Munger, J., Schelly, I., Morton, D.C., Noojipady, P., Barreto, P., Micol, L., Walker, N.F., Amazon, B., Cerrado, E., 2015. Brazil's Soy Moratorium. Science (80-. ). 347, 377-378. doi:10.1126/science.aaa0181

Grecchi, R.C.. B., Gwyn, Q.H.J.., Bénié, G.B.., Formaggio, A.R.., Fahl, F.C.., 2014. Land use and land cover changes in the Brazilian Cerrado: A multidisciplinary approach to assess the impacts of agricultural expansion. Appl. Geogr. 55, 300-312. doi:10.1016/j.apgeog.2014.09.014

Gaston, K. J., Pressey, R. L., \& Margules, C. R., 2002 Persistence and vulnerability: retaining biodiversity in the landscape and in protected areas. J. Biosci. 27, 361-84. Retrieved from http://www.ncbi.nlm.nih.gov/pubmed/12177535.

Haddad, N.M., Brudvig, L. a., Clobert, J., Davies, K.F., Gonzalez, a., Holt, R.D., Lovejoy, T.E., Sexton, J.O., Austin, M.P., Collins, C.D., Cook, W.M., Damschen, E.I., Ewers, R.M., Foster, B.L., Jenkins, C.N., King, a. J., Laurance, W.F., Levey, D.J., Margules, C.R., Melbourne, B. a., Nicholls, a. O., Orrock, J.L., Song, D.-X., Townshend, J.R., 2015. Habitat fragmentation and its lasting impact on Earth's ecosystems. Sci. Adv. 1, e1500052e1500052. doi:10.1126/sciadv.1500052

Han, X., Smyth, R.L., Young, B.E., Brooks, T.M., De Lozada, A.S., Bubb, P., Butchart, S.H.M., Larsen, F.W., Hamilton, H., Hansen, M.C., Turner, W.R., 2014. A biodiversity indicators dashboard: Addressing challenges to monitoring progress towards the Aichi biodiversity targets using disaggregated global data. PLoS One 9. doi:10.1371/journal.pone.0112046

Jennings, M.D., 2000. Gap analysis: concepts, methods, and recent results. Landscape Ecology. $15,5-20$.

Jepson, W., 2005. A disappearing biome? Reconsidering land-cover change in the Brazilian savanna. Geogr. J. 171, 99-111. doi:10.1111/j.1475-4959.2005.00153.x

Jepson, W., Brannstrom, C., Filippi, a, 2010. Access Regimes and Regional Land Change in the Brazilian Cerrado, 1972-2002. Ann. Assoc. Am. Geogr. 100, 87-111. doi:10.1080/00045600903378960

Kharouba, H.M., Kerr, J.T., 2010. Just passing through: Global change and the conservation of biodiversity in protected areas. Biol. Conserv. 143, 1094-1101. doi:10.1016/j.biocon.2010.02.002

Killeen, T.J., Calderon, V., Soria, L., Quezada, B., Steininger, M.K., Harper, G., Solórzano, L. a., Tucker, C.J., Sol+|rzano, L. a, 2007. Thirty years of land-cover change in Bolivia. Ambio 36, 600-6. doi:10.1579/0044-7447(2007)36[600:TYOLCI]2.0.CO;2

Klink, C. A. \& Moreira, A. G., 2002. Past and Current Human Occupation, and Land Use. In: The Cerrados of Brazil: Ecology and Natural History. 69-90. 
Klink, C. a., Machado, R.B., 2005. Conservation of the Brazilian Cerrado. Conserv. Biol. 19, 707-713. doi:10.1111/j.1523-1739.2005.00702.x

Lande's, R., 2003. Extinction Thresholds in Demographic Models of Territorial Populations. The American Naturalist.130, 624-635, 1987.

Lawler, J.O.J.L., Hite, D.E.W., 2003. Integrating representation and vulnerability: two approaches for prioritizing areas for conservation. Ecol. Appl. 13, 1762-1772.

Li, T., Shilling, F., Thorne, J., Li, F., Schott, H., Boynton, R., Berry, A.M., 2010. Fragmentation of China's landscape by roads and urban areas. Landsc. Ecol. 25, 839-853. doi:10.1007/s10980-010-9461-6

Machado, R.B., M.B. Ramos Neto, P.G.P. Pereira, E.F. Caldas, D.A. Gonçalves, N.S. Santos, K. Tabor e M. Steininger, 2004. Estimativas de perda da área do Cerrado brasileiro. Relatório técnico não publicado. Conservação Internacional, Brasília, DF.

Machado, R.D.P., 2012b. Proposta metodológica para avaliação de impactes na paisagem. Dissertação de mestrado. Lisboa: Instituto Superior de Estatística e Gestão de Informação da Universidade Nova de Lisboa. 143p.

Machado, R.B., Souza Aguiar, L.M.de, Ramos Neto, M.B., Scaramuzza, C.A. de M., Bottura, G., F.Dias, J. Déstro, G.F.G., Bacellar-Schittini, A.E.de F. 2012b Os princípios da complementaridade e insubstituibilidade na revisão das áreas prioritárias para a conservação do Cerrado. 226-240. In: Conservação da Biodiversidade com SIG, São Paulo: Oficina de Textos.

Mas, J.-F., Kolb, M., Paegelow, M., Camacho Olmedo, M.T., Houet, T., 2014. Inductive patternbased land use/cover change models: A comparison of four software packages. Environ. Model. Softw. 51, 94-111. doi:10.1016/j.envsoft.2013.09.010

McGarigal, K. \& Marks, B. J. Fragstats Spatial Pattern Analysus Program for quantifying $\begin{array}{lllll}\text { landscape } & \text { structure. } & \text { Software. } & 1995 . & \text { Disponível }\end{array}$ http://www.umass.edu/landeco/pubs/mcgarigal.marks.1995.pdf.

Margules, C.R., Pressey, R.L., 2000. Systematic conservation planning. Nature 405, 243-53. doi: $10.1038 / 35012251$

Marini, Â.M., Barbet-massin, M., Esteves, L., Jiguet, F., 2009. Major current and future gaps of Brazilian reserves to protect Neotropical savanna birds. Biol. Conserv. 142, 3039-3050. doi:10.1016/j.biocon.2009.08.002

Metzger, J.-P., Décamps, H., 1997. The structural connectivity threshold: An hypothesis in conservation biology at the landscape scale. Acta Oecologica. doi:10.1016/S1146609X(97)80075-6

Miccolis, A., Andrade, R.M.T., Pacheco, P., 2014. Land-use trends and environmental governance policies in Brazil - Paths forward for sustainability. doi:10.17528/cifor/005435

Mittermeier, R. A., Myers, N., Thomsen, J. B., da Fonseca, G. A. B. \& Olivieri, S., 1998. Biodiversity hotspots and major tropical wilderness areas: approaches to setting conservation priorities. Conserv. Biol.12, 516-520. 
Mittermeier, R.A.; P. Robles-Gil; M. Hoffman; J. Pilgrim; T. Brooks, C.G. Mittermeier; J.F. Lamoreux; G.A.B. da Fonseca, 2004. Hotspots revisited: Earth's biologically richest and most endangered terrestrial ecoregions. CEMEX, Cidade do México, México.

Müller, R., Pistorius, T., Rohde, S., Gerold, G., Pacheco, P., 2013. Policy options to reduce deforestation based on a systematic analysis of drivers and agents in lowland Bolivia. Land Use Policy 30, 895-907. doi:10.1016/j.landusepol.2012.06.019

Myers, N.; Mittermeier, R. A.; Mittermeier, C. G.; da Fonseca, G. A.; Kent, J., 2000. Biodiversity hotspots for conservation priorities. Nature, 403, 853-858.

Noss, R.F., 1990. Indicators for Monitoring Biodiversity: A Hierarchical Approach. Conserv. Biol. 4, 355-364. doi:10.1111/j.1523-1739.1990.tb00309.x

Oliveira-Filho, A.T. \& Ratter, J.A., 2002. Vegetation physiognomies and wood flora of the bioma Cerrado. In: OLIVEIRA, P. S.; MARQUIS, R. J. (Eds.). In: The Cerrados of Brazil: ecology and natural history of a neotropical Savanna. New York: Columbia University Press,91-120.

Pérez-Vega, A., Mas, J.-F., Ligmann-Zielinska, A., 2012. Comparing two approaches to land use/cover change modeling and their implications for the assessment of biodiversity loss in a deciduous tropical forest. Environ. Model. Softw. 29, 11-23. doi:10.1016/j.envsoft.2011.09.011

Pontius, R.G., Gilmore Pontius, Robert, J., 2000. Quantification error versus location error in comparison of categorical maps. Photogramm. Eng. Remote Sensing. doi:Cited By (since 1996) 180 Export Date 10 May 2012

Pontius Jr.; Gil, R.; Schneider, L. C., 2004 Land-cover change model validation by an ROC method for the Ipswich watershed, Massachusetts , USA. Environment, 85, 239-248.

Prates, A.P.L.\& Irving, M. d. A., 2015. Conservação da Biodiversidade e Políticas Públicas para as Áreas Protegidas no Brasil: Desafios e Tendências da Origem da CDB às Metas de Aichi. Rev. Bras. Políticas Públicas 1, 28-58. doi:10.1017/CBO9781107415324.004

Pressey, R.L.;Taffs, K.H., 2001. Scheduling priority conservation action in production landscapes : priority areas in western New South Wales defined by irreplaceability and vulnerability to vegetation loss. Biological Conservation, 100, 345-376.

Pressey, R.L., Cowling, R.M., Rouget, M., 2003. Formulating conservation targets for biodiversity pattern and process in the Cape Floristic Region, South Africa. Biol. Conserv. 112, 99-127. doi:10.1016/S0006-3207(02)00424-X

Pressey, R.L., Cabeza, M., Watts, M.E., Cowling, R.M., Wilson, K. a, 2007. Conservation planning in a changing world. Trends Ecol. Evol. 22, 583-92. doi:10.1016/j.tree.2007.10.001

R Core Team , 2012. R: A language and environment for statistical computing. R Foundation for Statistical Computing, Vienna, Austria. ISBN 3-900051-07-0, URL http://www.Rproject.org/.

Ratter J.A., Dargie T.C.D., 1992. An analysis of the floristic composition of 26 cerrado areas in Brazil. Edinburgh Journal of Botany. 49, 235-250. 
Ratter, J.A.; Ribeiro, J.F. and Bridgewater, S., 1997. The Brazilian Cerrado vegetation and threats to its biodiversity. Bot. Brief. 223-230.

Rêdo, D., Aide, T.M., Clark, M.L., 2013. Vegetation change in Brazil 's dryland ecoregions and the relationship to crop production and environmental factors : Cerrado, Caating, and Mato Grosso, 2001 - 2009. J. Land Use Sci. 37-41.

Rodrigues, A.S.L., Andelman, S.J., Bakarr, M.I., Boitani, L., Brooks, T.M., Cowling, R.M., Fishpool, L.D.C., Fonseca, G.A.B., Gaston, K.J., Hoffmann, M., Long, J.S., Marquet, P.A., Pilgrim, J.D., Pressey, R.L., Schipper, J., Sechrest, W., Stuart, S.N., Underhill, L.G., Waller, R.W., Watts, M.E.J., Yan, X., Animale, B., 2004. Effectiveness of the global protected area network in representing species diversity. Nature 428, 9-12. doi:10.1038/nature02459.1.

Rosa, I.M.D., Purves, D., Carreiras, J.M.B., Ewers, R.M., 2014. Modelling land cover change in the Brazilian Amazon: temporal changes in drivers and calibration issues. Reg. Environ. Chang. 1-30. doi:10.1007/s10113-014-0614-z

Rosa, I.M.D., Souza, C., Ewers, R.M., 2012. Changes in Size of Deforested Patches in the Brazilian Amazon. Conserv. Biol. 26, 932-937. doi:10.1111/j.1523-1739.2012.01901.x

Rouget, M., Richardson, D.M., Cowling, R.M., Lloyd, J.W., Lombard, A.T., 2003. Current patterns of habitat transformation and future threats to biodiversity in terrestrial ecosystems of the Cape Floristic Region, South Africa. Biol. Conserv. 112, 63-85. doi:10.1016/S00063207(02)00395-6

Salmona, Y. B., 2010. Cerrado com C ou com S? Modelagem de cenários futuros para o bioma. Dissertação. 87p. Universidade de Brasília (DF), 87p.

Sano, E.E., Rosa, R., Brito, J.L.S., Ferreira, L.G., 2010. Land cover mapping of the tropical savanna region in Brazil. Environ. Monit. Assess. 166, 113-24. doi:10.1007/s10661-0090988-4

Shi, H., Singh, A., Kant, S., Zhu, Z., Waller, E., 2005. Integrating Habitat Status, Human Population Pressure, and Protection Status into Biodiversity Conservation Priority Setting. Conserv. Biol. 19, 1273-1285. doi:10.1111/j.1523-1739.2005.00225.x

Smith, J., Winograd, M., Gallopin, G., Pachico, D., 1998. Dynamics of the agricultural frontier in the Amazon and savannas of Brazil : analyzing the impact of policy and technology. Environ. Model. Assess. 3, 31-46.

Stan, K., Sanchez-Azofeifa, A., Espírito-Santo, M., Portillo-Quintero, C., 2015. Simulating deforestation in Minas Gerais, Brazil, under changing government policies and socioeconomic conditions. PLoS One 10, 1-19. doi:10.1371/journal.pone.0137911

Svancara, L.K., Brannon J., R., Scott, M., Groves, C.R., Noss, R.F., Pressey, R.L., 2005. Policydriven versus Evidence-based Conservation: A Review of Political Targets and Biological Needs. Bioscience 55, 989. doi:10.1641/0006-3568(2005)055[0989:PVECAR]2.0.CO;2

Silva, J. F.; Farinãs, M. R.; Felfili, J. M. \& Klink, C. A., 2006. Spatial heterogeneity, land use and conservation in the Cerrado region of Brazil. Journal of biogeography, 33, 536-548. 
Soares-Filho, B.S., Nepstad, D.C., Curran, L.M., Cerqueira, G.C., Garcia, R.A., Ramos, C.A., Voll, E., McDonald, A., Lefebvre, P., Schlesinger, P., 2006. Modelling conservation in the Amazon basin. Nature 440, 520-3. doi:10.1038/nature04389

Stewart, R.R. \& Possingham, H.P., 2005. Efficiency, costs and trade-offs in marine reserve system design. Environmental Modeling and Assessment, 10, 203-213.

Teixeira, E. C., 2012. A Convenção sobre Diversidade Biológica vinte anos depois: uma análise econômica ambiental dos caminhos percorridos e das perspectivas. Tese. 127p.

Turner, M.G., 1989. Landscape Ecology: The Effect of Pattern on Process. Annu. Rev. Ecol. Syst. 20, 171-197. doi:10.1146/annurev.es.20.110189.001131

UNEP-CDB, 2004. Programme of Work on protected areas - 7th Conference of Parties - Decision vii/28. p.22. Convention on Biological Diversity, Kuala Lumpur, Indonesia.

UNEP, 2012. Perspectivas do meio ambiente mundial. GEO-3. Universidade Livre da Mata Atlântica. 2004. Disponível em: http://www.wwiuma.org.br/geo_mundial_arquivos. Acesso em: fevereiro de 2012.

VanDerWal, J. Falconi, L. Januchowski, S. Shoo, L. Storlie, C., 2014. SDMTools: Species distribution modelling tools: Tools for processing data associated with species distribution modelling exercises. $\mathrm{R}$ package version 1.1-20. http://CRAN.Rproject.org/package=SDMTools, 2014.

Veldkamp, a, Lambin, E.., 2001. Predicting land-use change. Agric. Ecosyst. Environ. 85, 1-6. doi:10.1016/S0167-8809(01)00199-2

Verburg, P.H., Overmars, K.P., Huigen, M.G. a., de Groot, W.T., Veldkamp, a., 2006. Analysis of the effects of land use change on protected areas in the Philippines. Appl. Geogr. 26, 153 173. doi:10.1016/j.apgeog.2005.11.005

Verburg, R., Filho, S.R., Lindoso, D., Debortoli, N., Litre, G., Bursztyn, M., 2014. The impact of commodity price and conservation policy scenarios on deforestation and agricultural land use in a frontier area within the Amazon. Land Use Policy 37, 14-26. doi:10.1016/j.landusepol.2012.10.003

Weigand Jr., Silva, D. C. da, Oliveira e Silva, D. de., 2001. Metas de Aichi: Situação atual no Brasil. Brasília: UICN, WWF-Brasil e IPÊ, 67p.

Wolff, S., 2000. Legislação Ambiental Brasileira: Grau de Adequação à Convenção sobre Diversidade Biológica. Brasília: MMA, 88p. 


\section{MATERIAL SUPLEMENTAR}

Tabela S1: Síntese sobre as metas no âmbito da CDB e metas brasileiras - Resolução CONABIO n ${ }^{\text {o. }} 3$ (MMA, 2006), *Metas de Aichi (CDB, 2013).

\begin{tabular}{|c|c|c|c|}
\hline OBJETIVOS CDB & METAS DE AICHI & METAS BRASILEIRAS & INDICADORES DE AICHI \\
\hline $\begin{array}{c}\text { "Reduzir as pressões } \\
\text { diretas sobre a } \\
\text { biodiversidade e } \\
\text { promover o uso } \\
\text { sustentável". }\end{array}$ & $\begin{array}{l}\text { Meta 5: "Em 2020, a taxa de perda de } \\
\text { todos os hábitats naturais, incluindo } \\
\text { as florestas, deverá ser, pelo menos a } \\
\text { metade e sempre que possível, levada } \\
\text { para perto de zero, e a degradação e a } \\
\quad \text { fragmentação serão } \\
\text { significativamente reduzidas". }\end{array}$ & $\begin{array}{c}\text { "Redução na taxa de } \\
\text { desmatamento de 50\% no Bioma } \\
\text { Cerrado". }\end{array}$ & $\begin{array}{l}\text { "Tendências em proporção de } \\
\text { hábitats } \\
\text { degradados/ameaçados; } \\
\text { Tendências da extensão de } \\
\text { biomas, ecossistemas e } \\
\text { hábitats selecionados; } \\
\text { Tendências da condição e da } \\
\text { vulnerabilidade dos } \\
\text { ecossistemas; Tendências na } \\
\text { fragmentação dos hábitats } \\
\text { naturais". }\end{array}$ \\
\hline $\begin{array}{l}\text { "Melhorar a situação } \\
\text { da biodiversidade } \\
\text { protegendo } \\
\text { ecossistemas, espécies } \\
\text { e diversidade } \\
\text { genética". }\end{array}$ & $\begin{array}{c}\text { Meta 11: "Em 2020, pelo menos } 17 \% \\
\text { das áreas terrestres especialmente } \\
\text { áreas de particular importância para a } \\
\text { biodiversidade e serviços } \\
\text { ecossistêmicos, conservadas por um } \\
\text { sistema de áreas protegidas (e outras } \\
\text { áreas importantes para a } \\
\text { conservação), ecologicamente } \\
\text { representativo, satisfatoriamente } \\
\text { interligados".* }\end{array}$ & $\begin{array}{c}\text { "Proteção da biodiversidade } \\
\text { assegurada em pelo menos } 2 / 3 \\
\text { das Áreas Prioritárias para a } \\
\text { biodiversidade, por meio de } \\
\text { Unidades de Conservação, Terras } \\
\text { Indígenas e Territórios } \\
\text { Quilombolas". } \\
\text { "Pelo menos } 17 \% \text { do bioma } \\
\text { Cerrado protegido por Unidades } \\
\text { de Conservação do SNUC".* }\end{array}$ & $\begin{array}{l}\text { "Tendências na cobertura } \\
\text { representativa de áreas } \\
\text { protegidas e outras } \\
\text { abordagens baseadas em } \\
\text { áreas". }\end{array}$ \\
\hline
\end{tabular}

Tabela S2: Fontes das variáveis utilizadas nos modelos.

\begin{tabular}{|lll|}
\hline & Distância de estradas & DNIT. Departamento Nacional de Infraestrutura e Transporte, 2012. \\
& Altimetria & USGS, 2010 \\
ESTATÍCAS & Declividade & USGS, 2010 \\
& $\begin{array}{l}\text { Pluviosidade anual } \\
\text { acumulada }\end{array}$ & Camaron, Hijmans et al., 2005 \\
\hline DINÂMICAS & Desmatamentos (2002) & MMA, 2006 \\
\hline
\end{tabular}


$\mathrm{Na}$ janela do LCM, Change Maps, foram ignoradas transições $\leq 100$ células. Em Variable Transformation Utility, foi utilizada a transformação Evidence Likehood. Em Test and Selection Site and Driver Variables: Declividade (V de Cramer $\phi$ : 0,1226); Distância de todas as estradas ( $\phi: 0,9624)$; Distância de desmatamentos - t1 $(\phi: 0,9624)$; Altimetria $(\phi: 0,199)$ e Pluviosidade $(\phi: 0,2301)$. Em Run Transition Sub-Model, foram utilizados os seguintes parâmetros: MLP Neural Network, Max sample size: 508094; MLP neural network parameters: Use automatic training e Use dynamic leaning rate; Start learnig rate: 0,00125 (até 1,29898); End learning rate:0,000125 (1,95313), Número de nós: 5 . O modelo completou 10.000 interações, com taxa de acurácia de 83,88\%. O restante dos parâmetros foram default.

Figura S1: Calibragem do LCM do Idrisi

Foram utilizadas 10.000 repetições, nos modelos CDB2 e CDB4. Em CDB 2, o valor da borda foi multiplicado por 5E-5, e em CDB 4, por 0. Em Run Options, foi assinalada a opção Simulated Annealing e Iterative Improvement: Two Step Iterative Improvement. Foram utilizadas 10.000 .000 interações com Decréscimo de temperatura de 100.000 e assinalada a opção Adaptive Annealing.

Figura S2: Calibragem do Marxan 
Tabela S3: Classes de vegetação do Cerrado (Brasil, 1992)

\begin{tabular}{|c|c|c|}
\hline & SIGLA & CLASSE DE VEGETAÇÃO \\
\hline \multirow{7}{*}{ FLORESTAS ESTACIONAIS } & $\mathrm{Cb}$ & Floresta Estacional Decidual das Terras Baixas \\
\hline & $\mathrm{Cm}$ & Floresta Estacional Decidual Montana \\
\hline & Cs & Floresta Estacional Decidual Submontana \\
\hline & $\mathrm{Fa}$ & Floresta Estacional Semidecidual Aluvial \\
\hline & $\mathrm{Fb}$ & Floresta Estacional Semidecidual de Terras Baixas \\
\hline & Fm & Floresta Estacional Semidecidual Montana \\
\hline & Fs & Floresta Estacional Semidecidual Submontana \\
\hline \multirow{14}{*}{$\begin{array}{l}\text { ÁREAS DE TENSÃO } \\
\text { ECOLÓGICA }\end{array}$} & $\mathrm{Ta}$ & Savana-Estépica Arborizada \\
\hline & $\mathrm{Td}$ & Savana-Estépica Florestada \\
\hline & $\operatorname{Tg}$ & Savana-Estépica Gramíneo-Lenhosa \\
\hline & $\mathrm{Tp}$ & Savana-Estépica Parque \\
\hline & $\mathrm{Aa}$ & Floresta Ombrófila Aberta Aluvial \\
\hline & $\mathrm{Ab}$ & Floresta Ombrófila Aberta das Terras Baixas \\
\hline & As & Floresta Ombrófila Aberta Submontana \\
\hline & $\mathrm{Da}$ & Floresta Ombrófila Densa Aluvial \\
\hline & $\mathrm{Dm}$ & Floresta Ombrófila Densa Montana \\
\hline & Ds & Floresta Ombrófila Densa Submontana \\
\hline & Ml & Floresta Ombrófila Mista Alto-Montana \\
\hline & $\mathrm{Mm}$ & Floresta Ombrófila Mista Montana \\
\hline & $\mathrm{Rm}$ & Refúgio Montano \\
\hline & $\mathrm{Ea}$ & Estepe Arborizada \\
\hline \multirow{3}{*}{ FORMAÇÕES PIONEIRAS } & $\mathrm{Pa}$ & Formação Pioneira com Influência Fluvial e/ou Lacustre \\
\hline & $\mathrm{Pf}$ & Formação Pioneira com Influência Flúvio-Marinha \\
\hline & $\mathrm{Pm}$ & Formação Pioneira com Influência Marinha \\
\hline \multirow{4}{*}{ SAVANAS } & $\mathrm{Sa}$ & Savana Arborizada \\
\hline & $\mathrm{Sd}$ & Savana Florestada \\
\hline & $\mathrm{Sg}$ & Savana Gramíneo-Lenhosa \\
\hline & Sp & Savana Parque \\
\hline
\end{tabular}




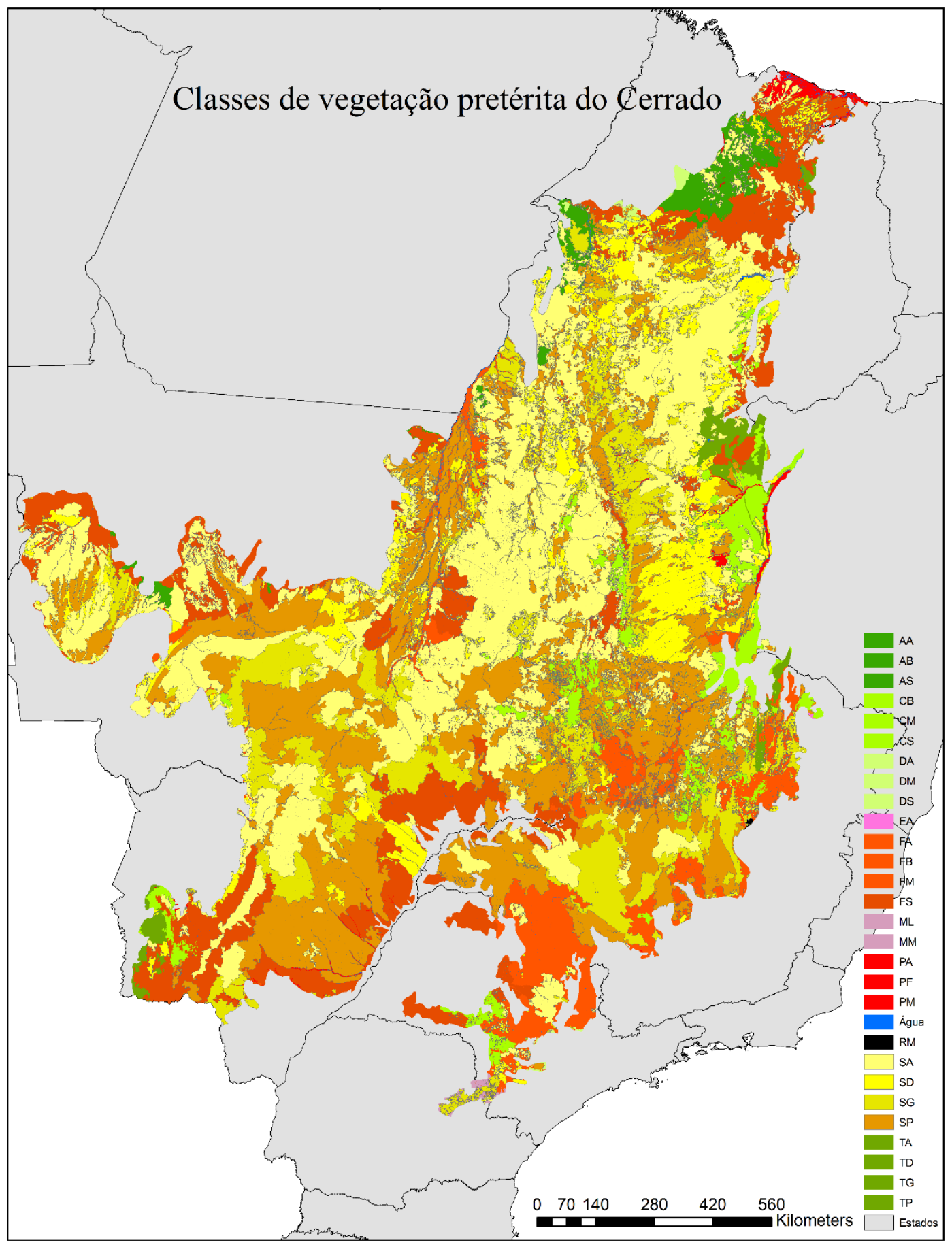

Figura S3: Classes de vegetação pretérita do Cerrado (Brasil, 2010) 
Tabela S4: Contribuição das principais métricas da paisagem na PCA.

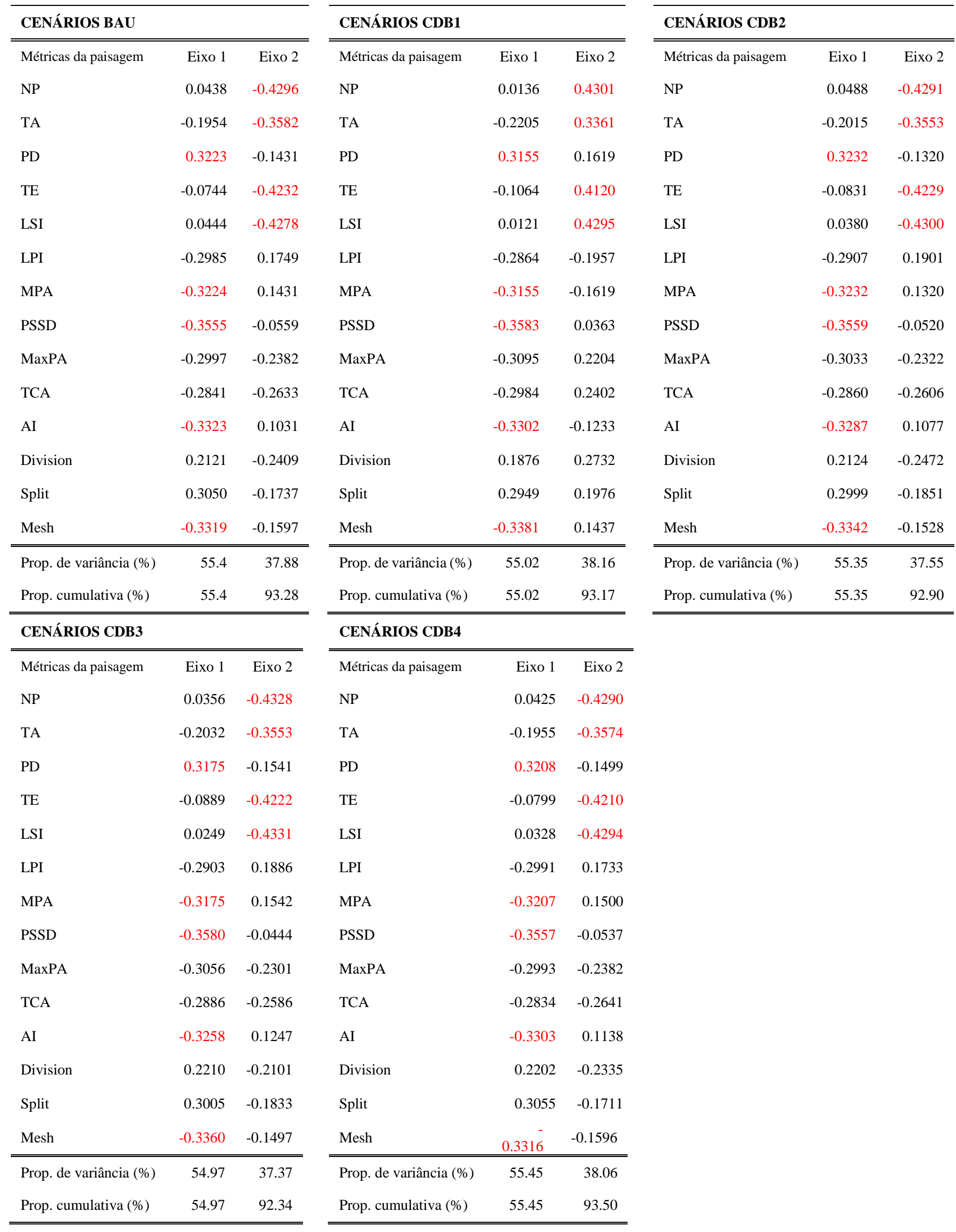




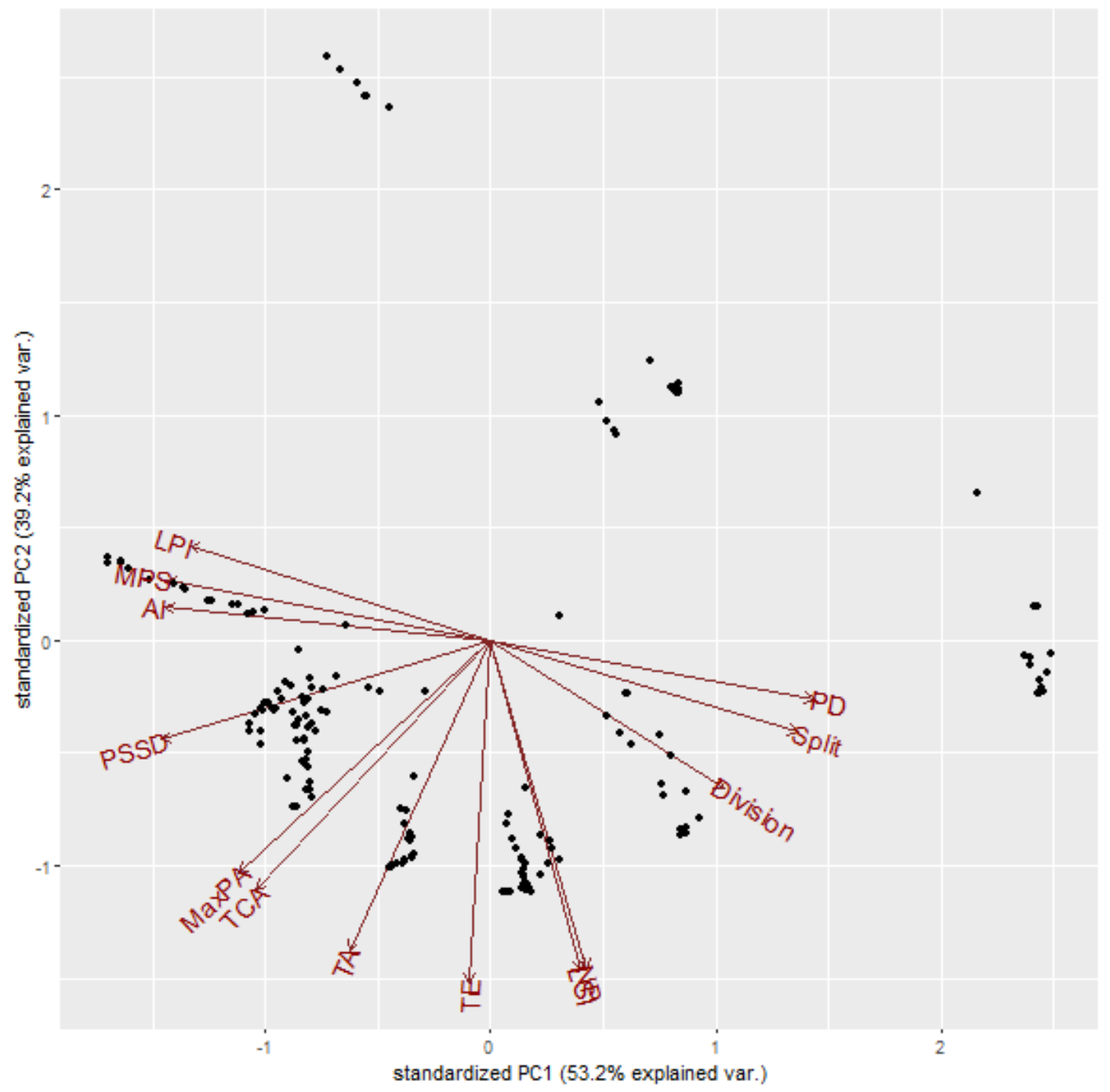

Figura S4: Gráfico representando os dois primeiros eixos da PCA de todos os cenários, em todos os anos e com todas as métricas: LPI (índice do maior fragmento); MPS (tamanho médio dos fragmentos); AI (índice de agregação); PSSD (desvio-padrão da área dos fragmentos); Mesh (tamanho efetivo da malha); MaxPA (área máxima do fragmento); TCA (área central total ); TA (área total); TE (total de bordas); LSI (índice de forma da paisagem); NP (número de fragmentos); Division (índice de divisão da paisagem); Split (índice de divisão) e PD (densidade de fragmentos) . 


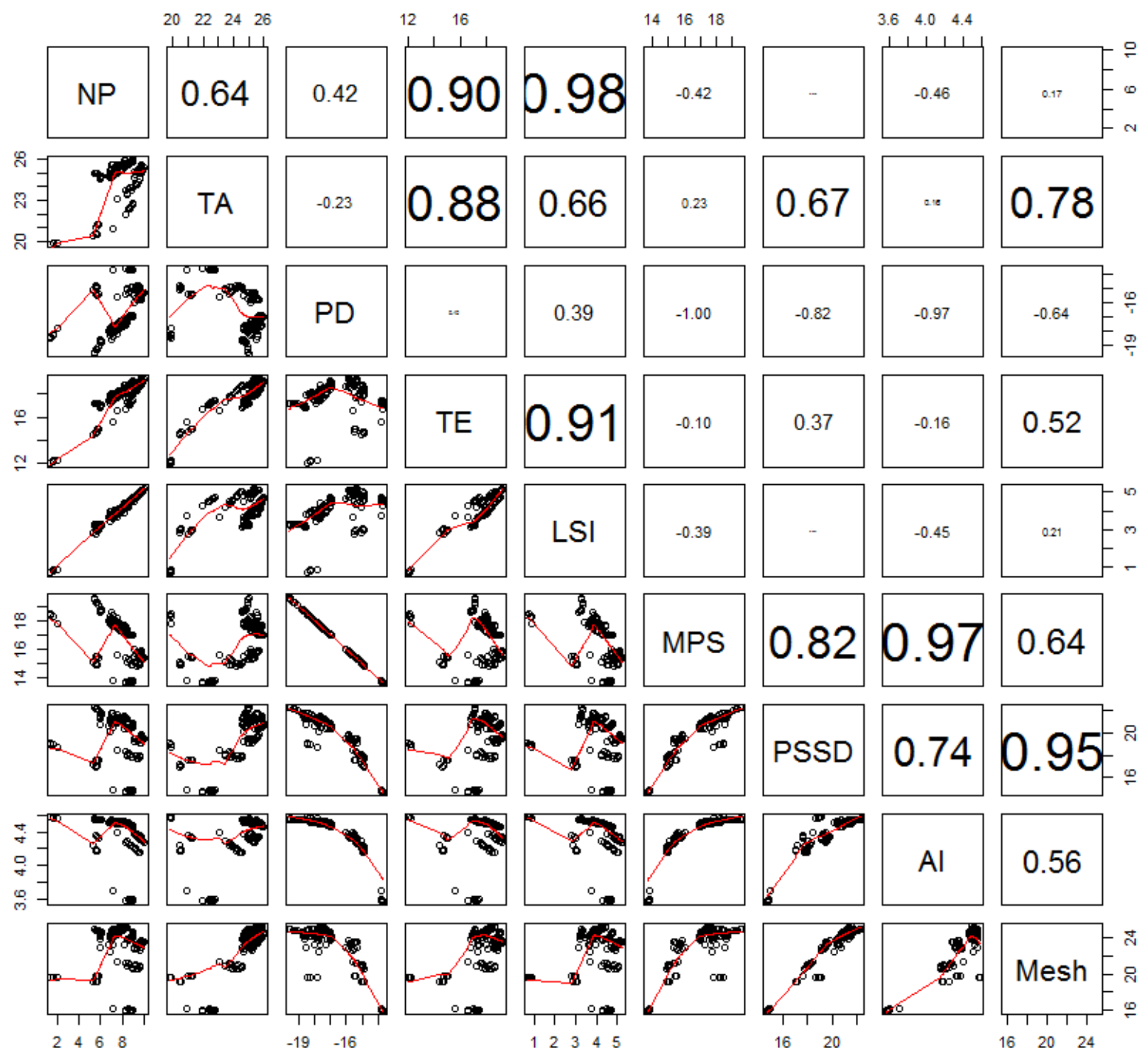

Figura S5: Gráfico de dispersão entre as métricas selecionadas e valores das análises de correlação de Spearman (em escala logarítmica); $p$-values $\leq 0,01$ (com exceção de NP versus $\mathrm{PSSD}=0,78 ;$ PD versus $\mathrm{TE}=0,12 ;$ TE versus $\mathrm{MPS}=0,12 ;$ TE versus $\mathrm{AI}=0,38 ; \mathrm{LSI}$ versus $\mathrm{PSSD}=0,3$ ). 
Tabela S2: Número de fragmentos agrupados por classes de tamanho em cada um dos cenários, período 2010 - 2050

\begin{tabular}{llllllll}
\hline CENÁRIOS & $\mathbf{1 1 0}$ & De 10 a 50 & De 50 a 100 & De 100 a 1000 & De 1000 a 10000 & $\leq \mathbf{1 0 0 0 0}$ & N. total \\
\hline BAU & 47561 & 1544 & 215 & 227 & 28 & 5 & 49580 \\
\hline CDB1 & 31104 & 1209 & 202 & 223 & 34 & 5 & 32777 \\
\hline CDB2 & 32789 & 1065 & 169 & 175 & 31 & 8 & 34237 \\
\hline CDB3 & 71966 & 1995 & 243 & 202 & 27 & 7 & 74440 \\
\hline CDB4 & 67430 & 1821 & 269 & 246 & 26 & 69799 \\
\hline
\end{tabular}

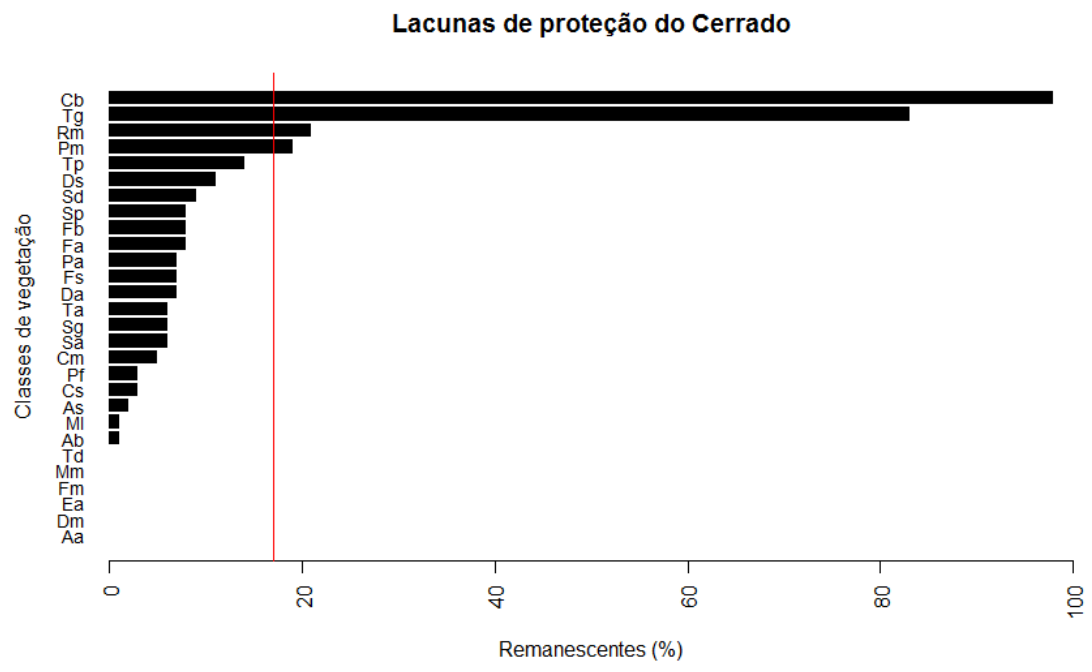

Figura S6: Análise de lacunas de proteção (17\%) por classe de vegetação em Unidades de Conservação de Proteção Integral e Terras Indígenas no Cerrado.
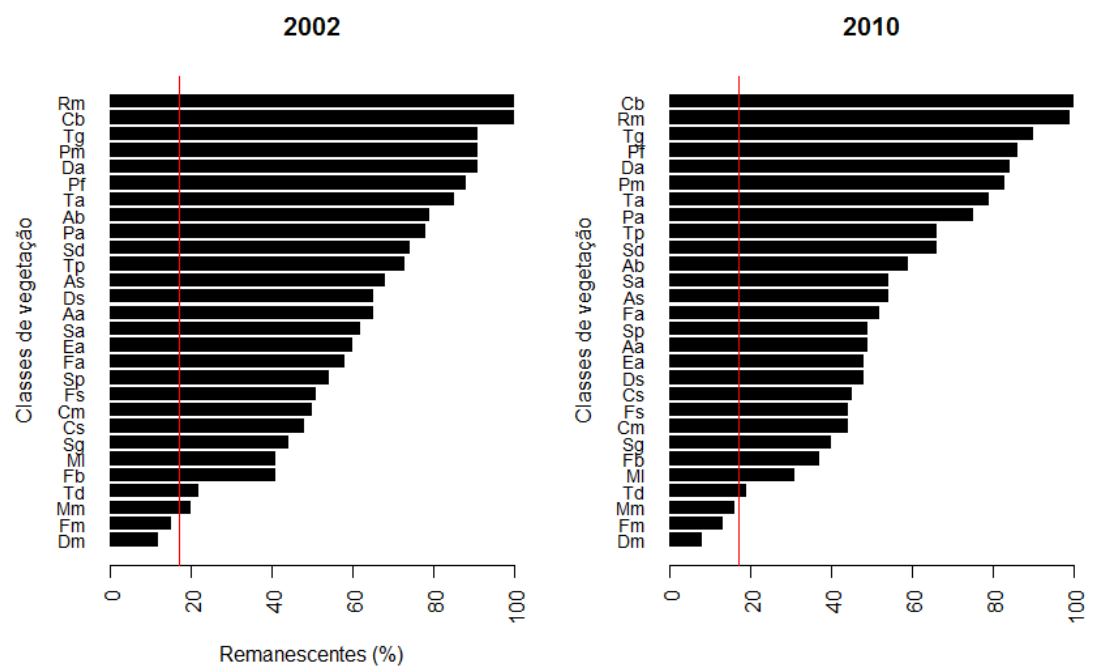

Figura S7: Remanescentes de Cerrado, distribuídos por classe de vegetação no Cerrado, anos 2002 e 2010. 

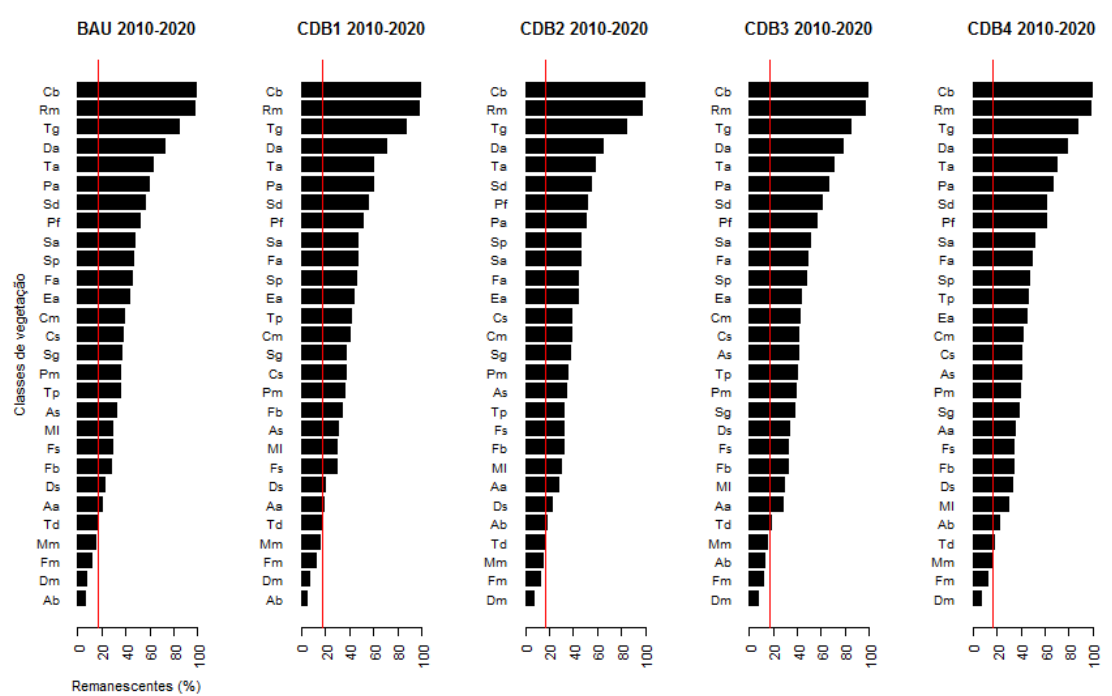

Figura S8: Remanescentes de Cerrado, distribuídos por classe de vegetação no Cerrado, nos cenários propostos, período de 2010-2020.
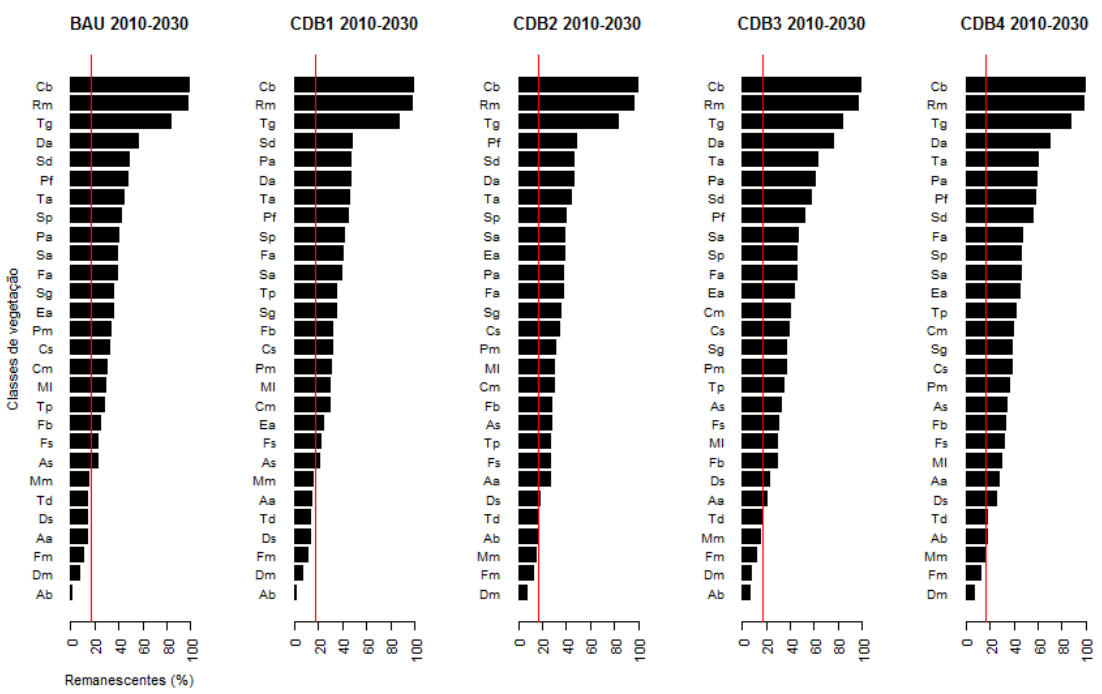

Figura S9: Remanescentes de Cerrado, distribuídos por classe de vegetação no Cerrado, nos cenários propostos, período de 2010-2030. 

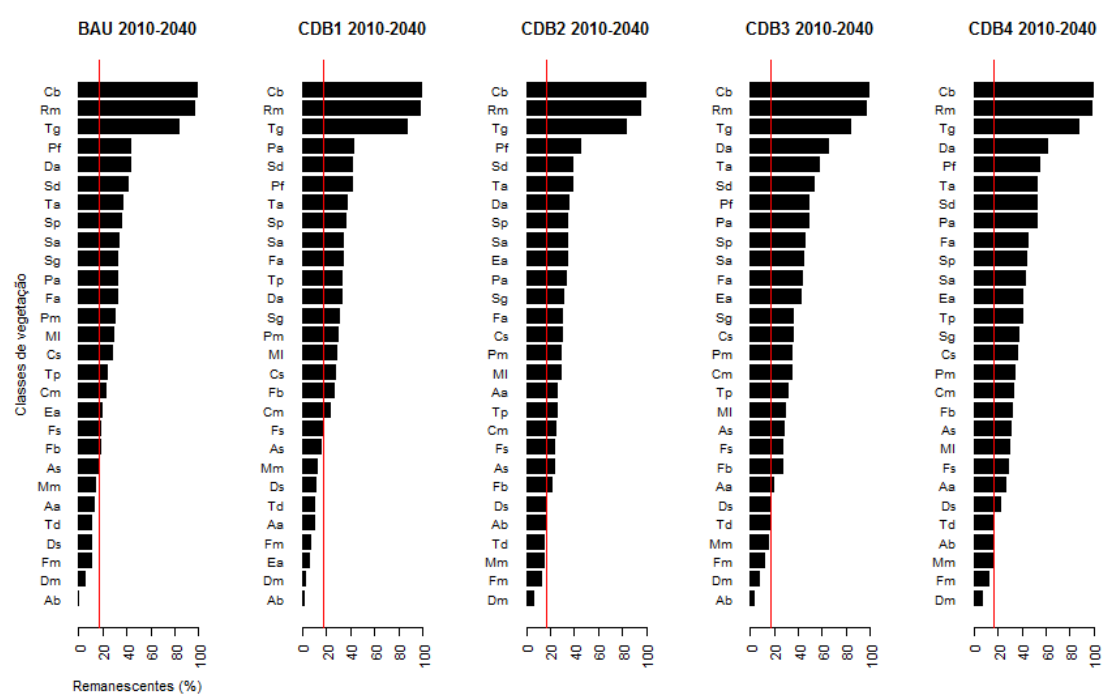

Figura S10: Remanescentes de Cerrado, distribuídos por classe de vegetação no Cerrado, nos cenários propostos, período de 2010-2040.

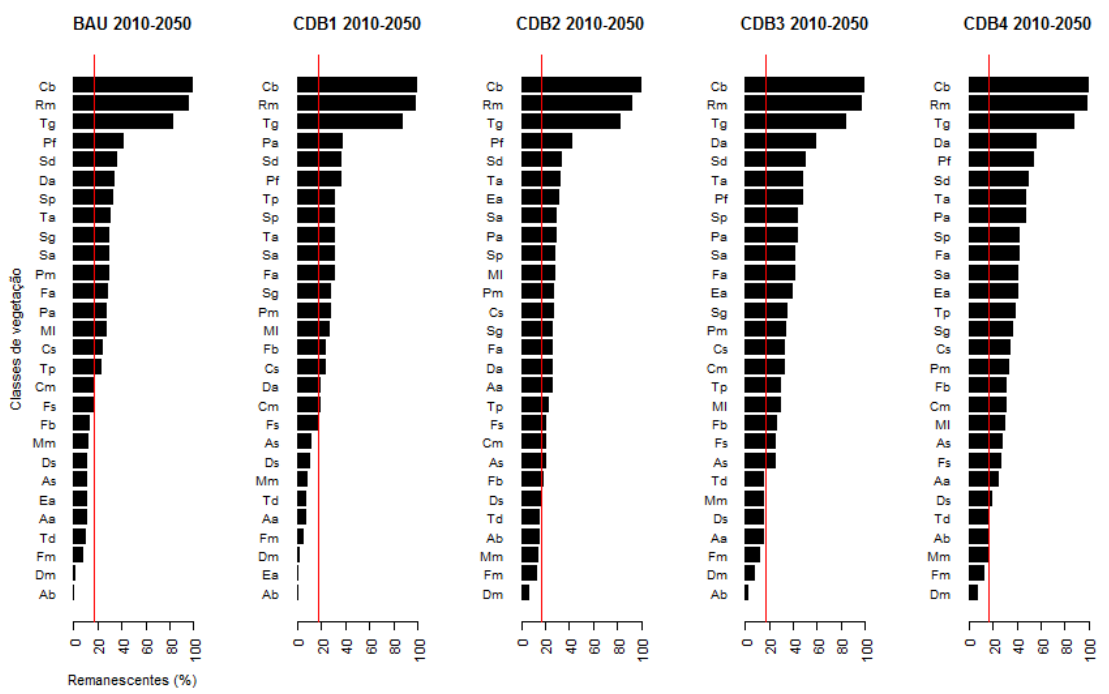

Figura S11: Remanescentes de Cerrado, distribuídos por classe de vegetação no Cerrado, nos cenários propostos, período de 2010-2050 


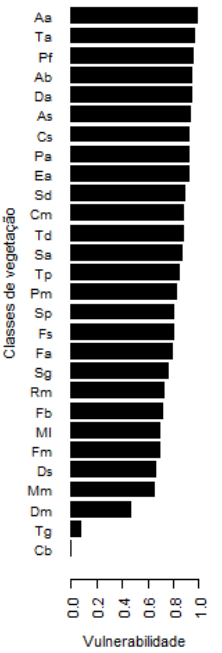

CDB1

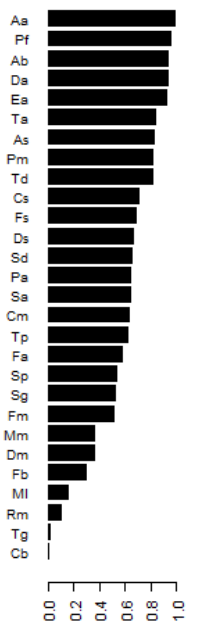

CDB2

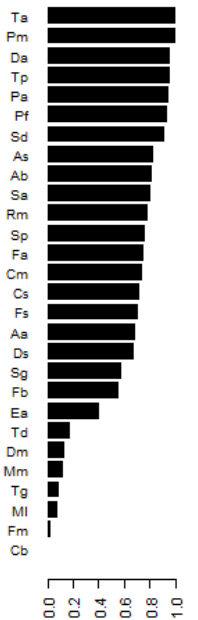

CDB3

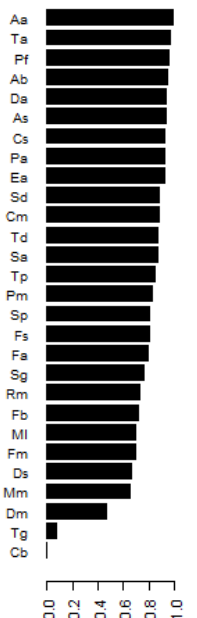

CDB4

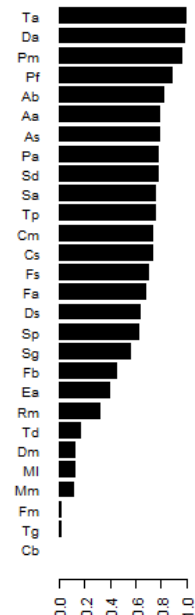

Figura S12: Distribuição da probabilidade de conversão de áreas naturais pelas classes de vegetação remanescentes de 2010 - 2020.

Tabela S6: Síntese dos padrões expressos pelos cenários propostos em relação ao Cenário BAU.

\begin{tabular}{|c|c|c|c|c|}
\hline & CDB1 & CDB2 & CDB3 & CDB4 \\
\hline Representatividade & $\begin{array}{l}\text { Diminui em } 10 \% \text { a perda } \\
\text { de alvos }\end{array}$ & $\begin{array}{l}\text { Diminui a perda dos alvo } \\
\text { em até } 70 \% \text {. }\end{array}$ & $\begin{array}{l}\text { Diminui a perda de alvos } \\
\text { em até } 30 \% \text {. }\end{array}$ & $\begin{array}{l}\text { Diminui a perda de alvos } \\
\text { em até } 70 \% \text {. }\end{array}$ \\
\hline Desmatamento & Mantém a perda. & Mantém a perda. & Diminui a perda. & Diminui a perda. \\
\hline Vulnerabilidade & $\begin{array}{l}\text { Altera pouco o padrão de } \\
\text { desmatamento. Diminui a } \\
\text { probabilidade de } \\
\text { conversão das Formações } \\
\text { Pioneiras e Savanas. }\end{array}$ & $\begin{array}{l}\text { Concentração de } \\
\text { desmatametos desde o } \\
\text { extremo norte ao sudoeste } \\
\text { do bioma e no estado de } \\
\text { Mato Grosso. } \\
\text { Diminui a probabilidade } \\
\text { de conversão das } \\
\text { Florestas Estacionais. }\end{array}$ & $\begin{array}{l}\text { Concentra os } \\
\text { desmatamentos na porção } \\
\text { norte do bioma. Mantém a } \\
\text { probabilidade conversão } \\
\text { das fitofisionomias. }\end{array}$ & $\begin{array}{l}\text { Concentra os } \\
\text { desmatamentos na porção } \\
\text { norte do bioma. Diminui a } \\
\text { probabilidade de conversão } \\
\text { das Formações Pioneiras e } \\
\text { Savanas. }\end{array}$ \\
\hline Fragmentação & $\begin{array}{l}\text { Diminui o número de } \\
\text { fragmentos (NP), o total de } \\
\text { bordas (TE) e a densidade } \\
\text { média (PD); aumenta o } \\
\text { tamanho médio dos } \\
\text { fragmentos (MPS) e os } \\
\text { torna menos homogêneos } \\
\text { (PSSD); aumenta o } \\
\text { isolamento dos fragmentos } \\
\text { (AI), diminui o índice de } \\
\text { forma da paisagem (LSI) e } \\
\text { mantém a conexão da } \\
\text { paisagem (Mesh). }\end{array}$ & $\begin{array}{l}\text { Diminui o número de } \\
\text { fragmentos (NP), o total } \\
\text { de bordas (TE) e a } \\
\text { densidade média (PD); } \\
\text { aumenta o tamanho médio } \\
\text { dos fragmentos (MPS) e } \\
\text { os torna menos } \\
\text { homogêneos (PSSD); } \\
\text { aumenta o isolamento dos } \\
\text { fragmentos (AI), diminui } \\
\text { o índice de forma da } \\
\text { paisagem (LSI) e torna a } \\
\text { paisagem mais conectada } \\
\text { (Mesh). }\end{array}$ & $\begin{array}{l}\text { Aumenta o número de } \\
\text { fragmentos (NP), o total de } \\
\text { bordas (TE) e diminui a } \\
\text { densidade média (PD); } \\
\text { aumenta o tamanho médio } \\
\text { dos fragmentos (MPS) e os } \\
\text { torna menos homogêneos } \\
\text { (PSSD); diminui o } \\
\text { isolamento dos fragmentos } \\
\text { (AI), aumenta o índice de } \\
\text { forma da paisagem (LSI) e } \\
\text { torna a paisagem mais } \\
\text { conectada (Mesh). }\end{array}$ & $\begin{array}{l}\text { Aumenta o número de } \\
\text { fragmentos (NP), o total de } \\
\text { bordas (TE) e diminui a } \\
\text { densidade média (PD); } \\
\text { aumenta o tamanho médio } \\
\text { dos fragmentos (MPS) e os } \\
\text { torna menos homogêneos } \\
\text { (PSSD); diminui o } \\
\text { isolamento dos fragmentos } \\
\text { (AI), aumenta o índice de } \\
\text { forma da paisagem (LSI) e } \\
\text { torna a paisagem mais } \\
\text { conectada (Mesh). }\end{array}$ \\
\hline
\end{tabular}



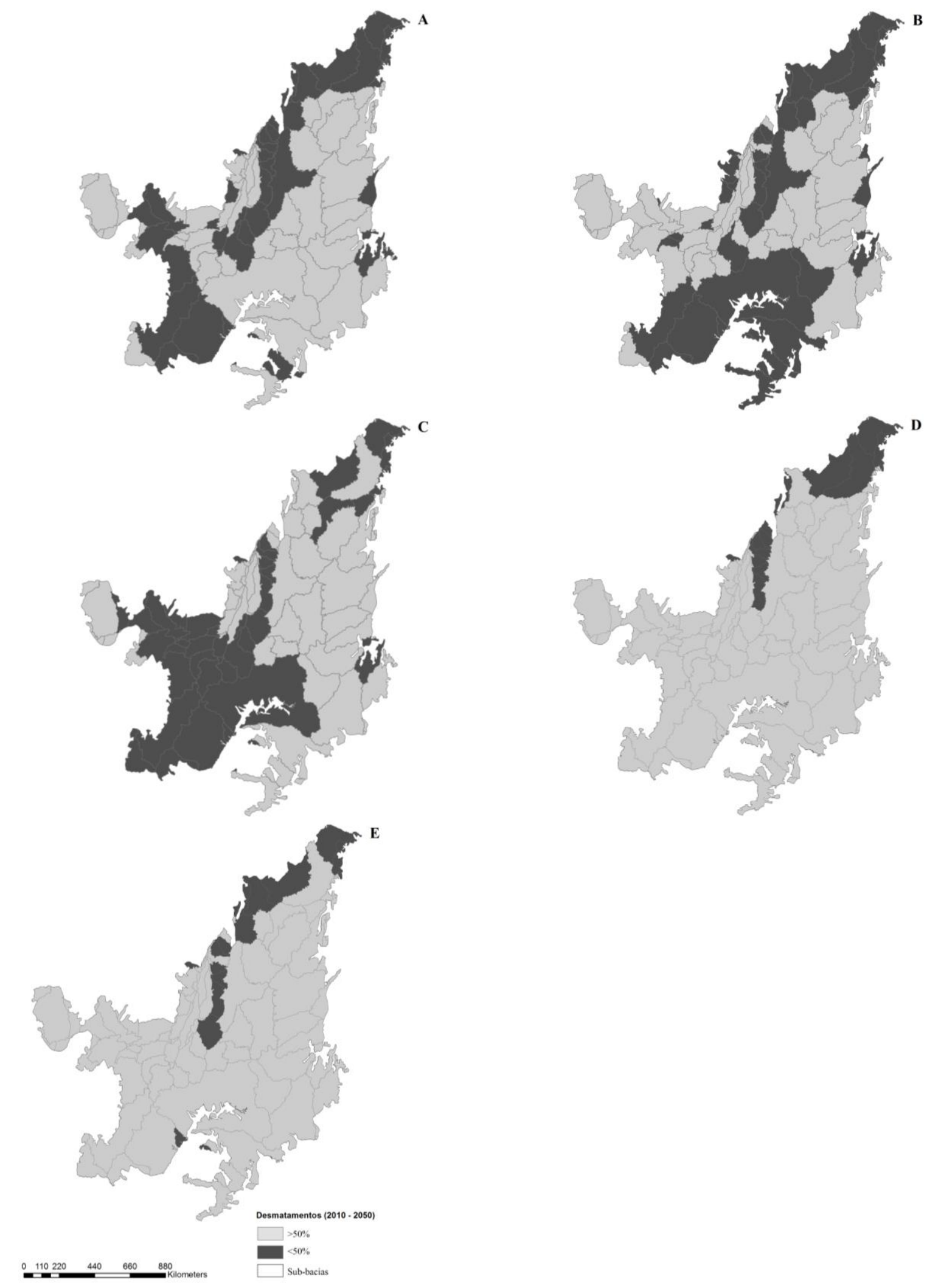

Figura S13: Porcentagem de área desmatada entre 2010 e 2050 por sub-bacia hidrográfica do Cerrado no cenários: A - BAU, B - CDB1, C - CDB2, D - CDB3 e E - CDB4. 


\title{
CAPÍTULO 3
}

\section{ESPÉCIES AMEAÇADAS E ENDÊMICAS DO CERRADO E AS METAS PARA A CONVENÇÃO SOBRE DIVERSIDADE BIOLÓGICA - CDB.}

\begin{abstract}
RESUMO
Avaliamos o progresso de algumas metas estipuladas pelo Brasil em virtude da CDB, utilizando as espécies ameaçadas e endêmicas do Cerrado como modelos. Aplicamos o Red List Index (RLI), para mostrar as tendências de conservação das espécies ameaçadas de extinção ao longo do tempo. Como indicador de pressão, utilizamos modelos de LUCC gerados para os anos de 2020 e 2050. Em cada período definimos cenários conservadores (BAU) e de governança (GOV.). Para inferir sobre a perda de hábitat das espécies, utilizamos modelos de distribuição de espécies (SDMs), associados ao mapeamento de uso do solo e aos cenários futuros gerados. Estes modelos também foram utilizados para desenhar redes prioritárias para a conservação no Cerrado e para avaliar como os SDMs das espécies-alvo são representados em um cenário de conservação onde as áreas protegidas alcançariam 17\% da área do Cerrado brasileiro. Nosso resultados demonstram a degradação no estado de conservação (IUCN, 2001), do conjunto dessas espécies, com RLI de 0,7209 em 2003 e 0,5595 em 2014, aproximando-se assim do seu limiar de extinção. Pela avaliação da perda de áreas de algumas destas espécies, em cenários futuros, inferiu-se que aquelas com SDMs menores e localizadas ao sul do bioma, necessitarão de ações urgentes de conservação. Por fim, o quantitativo de $17 \%$ de áreas deve ser ampliado para 23\% a fim de representar 17\% do SDM de todas as espécies-alvo.
\end{abstract}

PALAVRAS-CHAVE: Espécies ameaçadas; espécies endêmicas; Cerrado; mudanças no uso e cobertura da terra; Convenção sobre Diversidade Biológica. 


\section{INTRODUÇÃO}

A Convenção sobre Diversidade Brasileira (CDB) é um instrumento jurídico top-down, mas que não define regras, permitindo o livre desenvolvimento de estratégias de conservação aos seus países signatários (Prates \& Irving, 2015). Para o Brasil, significativos avanços foram alcançados quando são analisados os arcabouços jurídico e institucional construídos em torno da assinatura deste acordo, em 1998. Os avanços incluem a estruturação de programas de fomento e de desenvolvimento voltados ao tema biodiversidade (Brasil,1994 e Brasil,1998), marcos legais (Brasil, 2000; Brasil, 2002 ver Prates \& Irving, 2015 para demais informações) e a internalização de metas nacionais (Brasil, 2006; Brasil, 2013).

Durante a $10^{\mathrm{a}}$ Conferência das Partes (COP 10), foram estabelecidas metas: redução da perda, degradação e fragmentação dos hábitats naturais (meta 5); o aumento do percentual de proteção de áreas representativas para a conservação da biodiversidade e dos ecossistemas (Meta 11); a proteção das espécies contra a sua extinção e melhoria no seu status de conservação (Meta 12) e a recuperação de uma parte das áreas degradadas (Tabela S1).

Para mensurar o progresso das metas adotadas, há um aumento crescente da necessidade dos indicadores (Butchart et al., 2007; Han et al., 2014) (Tabela 1). O Red List Index (RLI) é um indicador de estado, para medir o risco de extinção global de um conjunto de espécies e acompanhar as mudanças nas suas categorias de riscos ao longo do tempo (Butchart et al., 2007). Baseado na Lista Vermelha de Espécies Ameaçadas (IUCN, 2001), que sintetiza informações como a dinâmica populacional, a distribuição das espécies (Extensão de Ocorrência - EOO e Área de Ocupância - AOO) e os impactos na perda de hábitats (Butchart et al., 2012). A vantagem de RLI é agrupar todas essas informações em um único índice de probabilidade de sobrevida global, permitindo comparações entre diferentes abrangências geográficas (Butchart at al, 2012).

Espécies ameaçadas são comumente utilizadas como indicadores por agências ambientais pelo mundo (Sarkar \& Illoldi-Rangel, 2010), pois podem prover informações sobre a conservação de espécies não-ameaçadas, indicando tendências (Drummond et al., 2010, De Heer et al., 2005) e geralmente, estão em áreas com forte pressão antrópica (Brooks et al., 2006). Já as espécies endêmicas estão concentradas em termos geográfico, possuindo poucas opções espaciais para sua conservação (Diniz-Filho et al., 2008) por isso, tendem a ser mais vulneráveis (Myers et al., 2000; Pimm et al., 2014).

A principal fonte de vulnerabilidade à perda de espécies, é a degradação e diminuição de hábitats (Fahrig, 2001) pela sua conversão para usos antrópicos (Verburg et al., 2006; Pressey et al., 2007). A mensuração das taxas e compreensão dos padrões espaciais das mudanças na paisagem exigem a utilização 
de modelos, tais como aqueles baseados em redes neurais, que projetam as tendências futuras de uma maneira explícita (ClarkLabs, 2009). Assim, esses algoritmos podem ser utilizados para observar as mudanças no uso do solo e cobertura da terra (Land Use Cover Change - LUCC) a partir de cenários prospectivos (Pérez-Vega et al., 2012). Os modelos de distribuição de espécies (Species Distribution Model - SDM) provêm informações sobre as EOO e AOO das espécies, uma vez que os registros de presença são incompletos (Rondinini et al., 2006; Phillips et al., 2006). Aliando estas informações é possível avaliar como as decisões sobre o uso do solo afetam a distribuição das espécies-alvo.

A persistência (Cowling et al., 1999; Araújo \& Williams, 2000; Cabeza \& Moilanen, 2001) e a representatividade (Noss, 1990; CDB, 1992; Fairbanks et al., 2001) são características desejáveis no processo de seleção de reservas (Margules \& Pressey, 2000; Sarkar \& Illoldi-Rangel, 2010), pois podem garantir a manutenção dos processos ecológicos e a conservação das espécies por muitas gerações. Associar informações espaciais sobre objetivos explícitos de conservação com a probabilidade de seu comprometimento pela perda ou degradação de hábitats, permite aos planejadores tomar decisões estratégicas sobre onde e quando alocar os poucos recursos normalmente destinados à conservação da biodiversidade (Pressey \& Taffs, 2001). Bird et al. (2012) e (Mello, 2014) utilizando SDMs de espécies-alvo e cenários futuros, na Amazônia e no Cerrado, respectivamente, concluíram que muitas espécies teriam uma degradação de seu status de conservação (IUCN, 2001) e, com estas informações também desenharam áreas de crise, refúgio e insubstituibilidade para estes alvos.

Nosso objetivo foi avaliar o progresso das metas estipuladas pelo Brasil em virtude da CDB quanto à proteção das espécies ameaçadas de extinção. Escolhemos o Cerrado como modelo e selecionamos espécies de vertebrados terrestres endêmicos deste bioma para avaliar seus status de proteção. 1) Delimitamos as espécies endêmicas e comparamos com a lista nacional de espécies ameaçadas. 2) Calculamos o RLI por classe de vertebrados, comparando as listas nacionais de espécies ameaçadas (Brasil, 2003 e Brasil, 2014) para todo o Brasil e, quanto sua ocorrência e endemismo no Cerrado. 3) Elaboramos SDMs dessas espécies-alvo e, comparamos com os mapas de uso do solo, 2002 e 2010 e os modelos LUCC para 2020 e 2050, para cenários conservador (BAU) e governança (GOV.) (capítulo 2) e avaliamos sua potencial perda de hábitat. 4) Analisamos a eficácia da meta de 17\% para a conservação (neste mesmo percentual) do SDMs das espécies-alvo; 5) Desenhamos redes de conservação para delimitar um subconjunto de áreas com alta prioridade para estas espécies, em cenários futuros. 


\section{METODOLOGIA}

\section{1. Área de estudo}

O Cerrado, de acordo com delimitação oficial (Brasil, 2004), tem área aproximada de 2 milhões de $\mathrm{Km}^{2}$, sendo o segundo maior bioma brasileiro em extensão territoral (Fig.1). Esta área nuclear está localizada na porção central do Brasil. A distribuição de sua vegetação remanescente é altamente heterogênea, sua porção sul, é bastante antropizada e fragmentada, estando os maiores fragmentos, principalmente, na parte norte do bioma (Sano et al., 2010). A riqueza de espécies está concentrada principalmente no sudoeste do bioma (Diniz-Filho et al., 2008), mas estudos prospectivos sugerem que esta tendência pode ser desviada para a porção norte com o aumento do número de inventários das áreas nativas (Bini et al., 2006). O Platô do Espinhaço e os vales dos rios Araguaia e Paranã são áreas com grande concentração de endemismos para as aves (Silva \& Bates, 2002). Mas o nível de endemismos de vertebrados é considerado baixo, em relação à outros biomas (Diniz-Filho et al., 2008), à exceção dos répteis e anfíbios (Colli et al, 2002; Nogueira et al, 2011). Atualmente, o Cerrado ainda é um dos biomas brasileiros mais ameaçados. Sua área remanescente de vegetação, em 2011, ocupava cerca de 50\%, e a taxa desmatamento anual era de 7.246,67 $\mathrm{Km}^{2}$ ano $^{-1}$, a maior taxa dentre os seis biomas brasileiros (Brasil, 2011). As maiores ameaças ao Cerrado são: a criação de gado, com pastagens nativas e plantadas e a agricultura mecanizada, principalmente, a soja (Klink \& Machado, 2005). No bioma, a soja substitui as áreas de pastagem, que ocupam novas áreas (Brandão et al., 2006; Rêdo et al., 2013), estima-se que mais de $200.000 \mathrm{Km}^{2}$ sejam suscetíveis à conversão pela soja. De acordo com o código florestal (Brasil, 2012) $110.000 \mathrm{Km}^{2}$ ainda podem ser legalmente cultivadas (Gibbs et al., 2015). As porções do Cerrado são desigualmente ocupadas, ao sul estão as áreas mais consolidadas e ao norte as maiores extensões de áreas remanescentes (Brannstrom et al., 2008). 


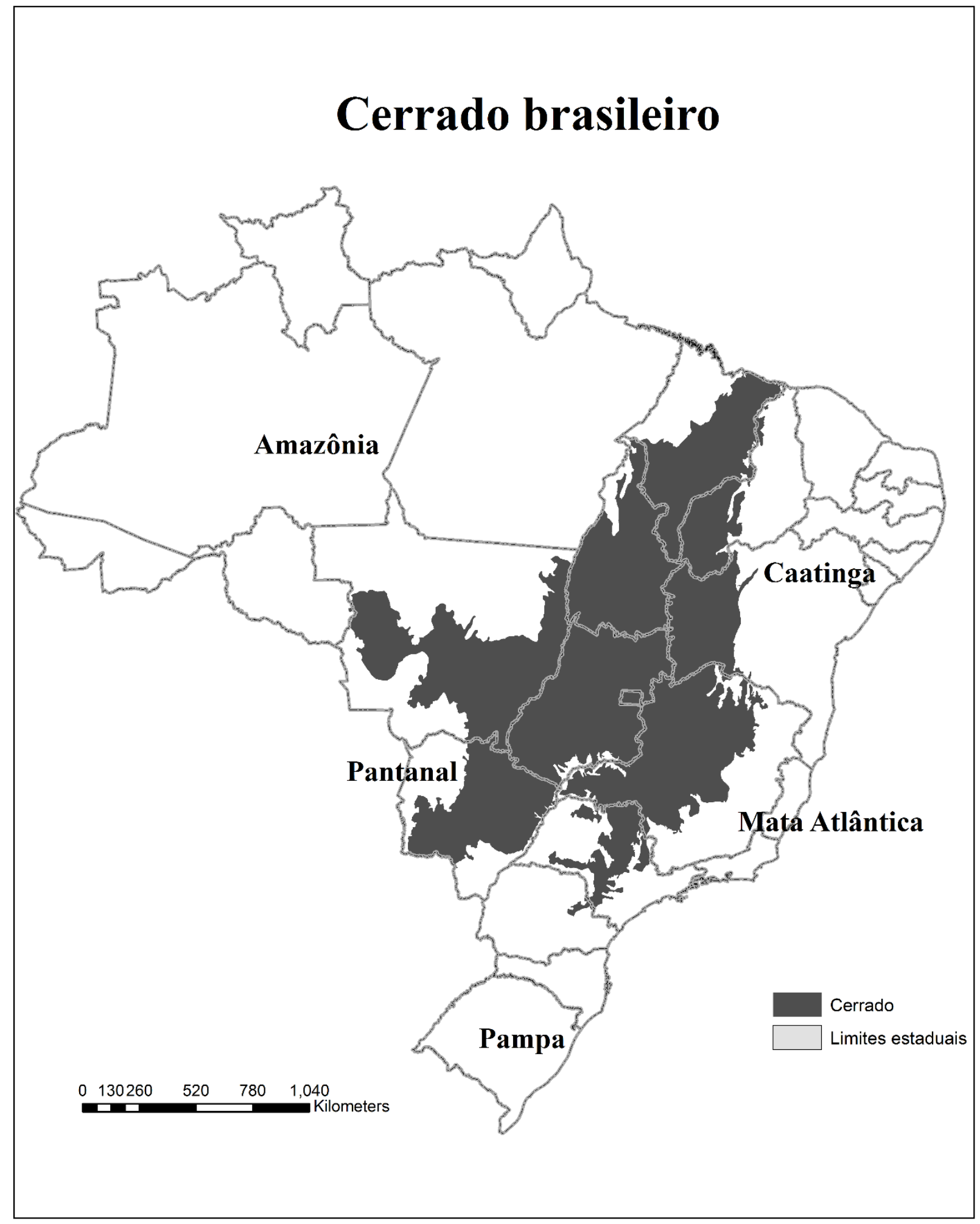

Fig. 1 -Área de estudo: O limite do Cerrado brasileiro (segundo Brasil, 2004), estados e biomas brasileiros. 


\section{2. Índice da lista vermelha (Red List Index - RLI)}

O RLI é uma medida da tendência positivas ou negativas do status de conservação de um conjunto de espécies analisadas. $\mathrm{O}$ índice considera a proporção de espécies em cada categoria de risco da IUCN e as mudanças válidas desta proporção ao longo do tempo (Butchart et al., 2007). Utilizando a lista de espécies ameaçadas de 2003 (Brasil, 2003) e 2014 (Brasil, 2014) e lista de espécies NT e DD de 2003 (Machado et al., 2005) e 2014 (ICMBio, 2014), calculamos o RLI geral e para cada táxon selecionado, considerando sua abrangência nacional, sua ocorrência no Cerrado e endemismo no bioma. Apenas espécies pertencentes as duas listas geradas com os mesmos critérios podem ser comparadas e, espécies com dados incompletos (DD) não entram no cálculo de acordo com a metodologia proposta. Espécies ameaçadas na lista de 2014 foram consideradas ameaçadas em 2003, com a exceção daquelas descritas a partir de 2003. O cálculo, de acordo com a equação (1) de Butchart (2007):

$$
\begin{aligned}
& R L I=1-\left(\frac{T}{M}\right) \\
& M=W_{\max } * N \text {, onde } W_{\max }= \\
& \text { máxima pontuação de status na Lista Vermelha de espécies ameaçadas da IUCN (Ex e EW = 5; CR = } \\
& 4 ; \mathrm{EN}=3 ; \mathrm{VU}=2 ; \mathrm{NT}=1 ; \mathrm{LC}=0 \text { ) e } N=\text { número de espécies avaliadas. } \\
& T=\sum W_{c} * N_{c(t) .}
\end{aligned}
$$

A escala varia de 0 a 1, valores perto de 0 indicam maior deterioração do status de conservação dessas espécies.

\subsection{Seleção das espécies-alvo}

Para este estudo selecionamos 47 espécies-alvo. Para a definição de espécies endêmicas do Cerrado, consultamos a literatura especializada, pois este tipo de delimitação pode variar, seja pela divergência quanto à delimitação geográfica do bioma Cerrado, por revisões atualizadas da distribuição geográfica das espécies ou pela sua classificação taxonômica (Lopes, 2009). O recorte do bioma considerado por este estudo leva em consideração a área núcleo do Cerrado brasileiro (Brasil, 2004). Nós selecionamos apenas as espécies endêmicas e ao mesmo tempo, ameaçadas de extinção de acordo com a Portaria $\mathrm{n}^{\text {o. }} 444$ de 17/12/2014 (Brasil, 2014) ou quase ameaçadas (ICMBio, 2014). Os grupos taxonômicos considerados foram os anfíbios, répteis, aves e mamíferos.

Para anfíbios consultamos o levantamento realizado por Valdujo et al. (2012), que consideraram espécies com pelo menos $30 \%$ de sua distribuição no Cerrado, e concluíram que $72 \%$ das espécies associadas a este bioma são endêmicas. Contudo, apenas 3 espécies estão ameaçadas de acordo com o Brasil (2014), já que excluímos Allobates brunneus pois numerosos espécimes vêm sendo catalogados na 
Amazônia (Lima et al., 2009) e sua área de distribuição é pequena no Cerrado quando comparado aquele bioma (Tab. S2).

No caso dos répteis (ordem Squamata) consultamos Nogueira et al. (2011), que registrou um alto índice de endemismo 39\% da riqueza regional dessas espécies $(n=103)$. Neste estudo, espécies marginais e com ocorrência em áreas de transição de Cerrado, também foram consideradas endêmicas. Destas consideramos 14 espécies de répteis endêmicos e ameaçados (Tab. S2).

Para as aves seguimos o estudo de Lopes (2009), que considerou 16 espécies como endêmicas, por terem 95\% de sua distribuição restrita no bioma Cerrado. Por entendermos que os critérios para a classificação de uma espécie como endêmica foram mais rigorosos que os de outros autores, como por exemplo (Silva \& Bates, 2002), optamos por incluir 29 espécies de outra categoria apresentada por Lopes (2009), aquelas “dependentes de conservação”. Estas, embora não endêmicas, têm $80 \%$ de sua distribuição dentro dos limites do bioma Cerrado ou 50\%, se consideradas ameaçadas ou com grande risco de perda hábitat. No total, foram 17 espécies de aves endêmicas e ameaçadas. Sporophila nigrorufa foi excluída pois têm área de ocorrência marginal ao Cerrado e Mergus octosetaceus porque tem sua área de distribuição associada aos ambientes aquáticos (Tab. S2).

Para avaliar espécies endêmicas de mamíferos, consideramos Paglia et al. (2012) e Bonvicino et al. (2012). Em Paglia et al. (2012) selecionamos apenas aquelas espécies com ocorrência restrita no bioma Cerrado. Bonvicino et al. (2012) corroboraram essas espécies, mas acrescentaram novas espécies a lista já compilada. Lonchophylla dekeyseri foi retirada da lista, pois novos registros dessa espécie foram encontrados na Caatinga (Leal et al., 2013). No total, selecionamos 13 espécies, endêmicas e ameaçadas (Tab. S2).

As informações sobre os alvos foram compiladas a partir de levantamento bibliográfico (artigos científicos, relatórios técnicos e outras publicações), bases de dados disponibilizadas on line, SpeciesLink (disponível em http://www.splink.org.br/index?lang=pt), Global Biodiversity Information Facility - GBIF (disponível em http://www.gbif.org/species) e consultas às bases de dados do IBAMA, do WWF, do ICMBio e de alguns pesquisadores da UnB.

\subsection{Modelagem de distribuição de espécies}

Utilizamos modelos de distribuição potencial de espécies, para minimizar os erros de omissão dos registros de localidade, que trazem o viés na representação da espécie (Rondinini et al., 2006; Grand et al., 
2007; Carvalho et al., 2010) o que reduz a eficiência na seleção de áreas, direcionando as ações de conservação em geral, para áreas com melhor amostragem (Rondinini et al., 2006),

Para construção dos modelos de distribuição de espécies (SDMs) utilizamos o algoritmo Maxent versão 3.3.3k (disponível em http://www.cs.princeton.edu/ schapire/maxent/, Phillips et al.,2006). Para as iterações do Maxent utilizamos os parâmetros básicos, com exceção da inicialização aleatória para cada novo modelo e do parâmetro bootstrap para a produção de réplicas. Inicialmente utilizamos todos os registros de ocorrências de cada espécie e 15 variáveis bioclimáticas do Projeto WorldClim (Hijmans et al., 2005) com resolução de $\sim 1 \mathrm{~km}^{2}$ (30 arc segundos) (disponível em http://www.worldclim.org/), que sintetizam dados de temperatura e precipitação (Tab. S3). Todas as camadas foram recortadas para o Brasil. Nesta rodada inicial, selecionamos as variáveis climáticas que contribuíram acima de $25 \%$, usando o teste Jackknife (Nyári \& Reddy, 2013), de acordo com a média de 5 modelos e para cada umas das classes: mamíferos, aves, répteis e anfíbios (Tab. S3).

Avaliamos a distribuição dos registros das espécies quanto à variação dos parâmetros ambientais utilizados na escala de trabalho, retirando aqueles duplicados. Assim, 75\% dos registros foram selecionados aleatoriamente para a elaboração dos modelos pelo Maxent e o restante foi usado para teste dos modelos. A média de 15 repetições representa os SDMs.

A validação dos modelos que foi feita por meio da Característica de Operação do Receptor (ROC), que considera os acertos do modelo quanto à sensitividade (presenças) e a especificidade (ausências). A metodologia utilizada foi o ROC parcial (Peterson, Papes \& Soberón, 2008), que utiliza somente uma parte da área total, aquela correspondente às predições. Assim, utilizando os pontos de testes particionados em várias réplicas, são geradas as razões de ROC-AUC do modelo/AUC aleatória, onde razões > 1 indicam bons modelos. Para efetuar essas análises utilizamos o software PartialROC (Barve, 2008) e posteriormente realizamos o Teste $\mathrm{T}$ para avaliar se havia diferenças significativas entre as razões.

Maxent produz uma superfície contínua de adequabilidade da espécie entre 0 e 1 e, para criar mapas binários de presença e ausência, para cada uma das espécies, nós reclassificamos os SDMs utilizando o parâmetro "10 percentil training presence logistic threshold" (Urbina-Cardona \& Flores-Villela, 2010; Radosavljevic \& Anderson, 2014). As áreas dos SDMs foram ajustadas para o recorte do bioma Cerrado utilizado neste estudo e quando muito discrepantes foram ajustadas para áreas geográficas condizentes com a literatura consultada, já que o objetivo do nosso estudo foi avaliar a evolução do status de conservação dessas espécies. Procedemos uma análise de sensitividade utilizando os registros para validação do modelo. 
Comparamos os mapas binários das espécies com estes registros e calculamos a porcentagem de omissões dos SDMs.

Os mapas binários ajustados foram utilizados para o cálculo da perda de área das espécies, de acordo com os tempos pretéritos de uso do solo, 2002 e 2010 (Brasil, 2011), e para projeções futuras, 2010 - 2020 e 2010 - 2050, em cenários conservadores (BAU) e de governança (GOV.), com redução de 50\% na taxa de desmatamento, como descritos no capítulo 2.

\subsection{Prioridades para a proteção das espécies}

Utilizamos o software Zonation (Moilanen et al. 2005), para desenhar redes de proteção das espécies de vertebrados terrestres endêmicos e ameaçados do Cerrado e avaliar como as áreas de ocorrência das espécies endêmicas e ameaçadas do Cerrado estão representadas em frações dessa solução.

Para processar o modelo, utilizamos os SDMs das espécies-alvo, geradas pelo Maxent. Para as espécies que não possuíam ocorrências suficientes para gerar os SDMs, utilizamos a melhor informação disponível sobre sua distribuição, como polígonos da IUCN Nature Serve (disponível em http://www.natureserve.org/). Como última opção, traçamos Polígonos Mínimos Convexos (PMCs). Contudo, algumas espécies foram excluídas dessa análise pois não possuíam os polígonos e os escassos registros de distribuição não foram suficientes para traçar os PMCs. Para cada grupo de espécie foram estabelecidos pesos de acordo com o status de conservação (Leathwick et al., 2008), assim: NT=1, vulnerável=2, em risco=3, criticamente em perigo $=4($ IUCN, 2001).

Zonation é um algoritmo determinístico que produz uma priorização hierárquica das áreas disponíveis para a conservação considerando múltiplos alvos de biodiversidade e a complementaridade. Este software classifica cada unidade de planejamento (pixels em um mapa matricial) de acordo com sua importância relativa. Sucessivas iterações removem as unidades de planejamento (UPs) menos importantes, minimizando a perda marginal e os custos para a conservação. Selecionamos como regra de remoção de UPs, a opção Core-area Zonation (CAZ), que retém o maior valor proporcional de um alvo em uma determinada unidade de planejamento. Este modo de cálculo identifica áreas com maior importância para alvos raros, em detrimento às áreas com maior riqueza média de espécies. Seguindo a equação 2 (Moilanen et al., 2005; Moilanen, 2007):

$$
\delta_{1}=\max _{j} \frac{q_{i j w_{j}}}{c_{i}},
$$

Onde $\delta_{1}=$ perda marginal mínima de valor biológico para cada célula;

$w_{j}$ é o peso do alvo ${ }_{j}$ e $c_{i}$ é o custo para adicionar a UP $i$ à solução. 
Como método de agregação de reservas, selecionamos edge removal, que produz alguma conectividade e warp factor $=100$, que retira 100 células de uma vez, neste caso, $100 \mathrm{~km}^{2}$. Nós utilizamos as “Áreas prioritárias para a conservação da biodiversidade do Cerrado" (Brasil, 2013b) como uma referência de custo, aumentando sua coincidência com o ranking produzido neste estudo. Os custos foram estabelecidos de acordo com as recomendações de manejo para cada uma das áreas selecionadas, quanto menor o valor da área, mais relevância ecológica, e menor seu custo para a conservação. Assim, as unidades de conservação de proteção integral (UCPIs) existentes $=1$; áreas com recomendação para a criação e/ou expansão de $\mathrm{UCs}=2$; aquelas importantes para o uso sustentável $=3$; para o ordenamento territorial $=4$; com necessidade de inventário $=5$, e para o restante das áreas remanescentes não selecionadas para a priorização $=10$ (em caso de sobreposição de recomendações, consideramos aquelas com menor custo). As UCPIs foram utilizadas como hierarchical removal mask $=100$, integrando-as à solução final. Mask missing áreas, definem a área de estudo, neste caso, usamos os remanescentes de vegetação gerados pelos modelos LUCC (capítulo 2) para BAU 2010-2020 e 2010-2050 e GOV. 2010-2020 e 2010-2050, para cada um dos cenários gerados.

As análises espaciais e estatísticas foram realizadas utilizando os softwares R (R Core Team, 2012) e ArcGis 10.3.1 (ESRI, 2015).

\section{RESULTADOS}

\subsection{Red List Index}

No total 526 espécies de vertebrados (excluindo peixes) foram comparadas entre as listas de 2003 e de 2014 (Tab. S5), com RLIs 0,72 e 0,56. Os resultados dos cálculos do RLI para todas as espécies de ampla ocorrência (aves, mamíferos, anfíbios e répteis) demonstraram o aumento da tendência na taxa de perda da biodiversidade (Fig. 2A e Tab. S4), que é mais acentuada quando delimitamos sua extensão de ocorrência àquelas presentes no Cerrado, e para as endêmicas desse bioma (Figs. 2 B-E). Espécies descritas a partir de 2003 (8 anfíbios, 3 répteis, 2 mamíferos e 10 aves e, as espécies consideradas DD em 2003 e/ou 2014) não foram utilizadas nos cálculos. 


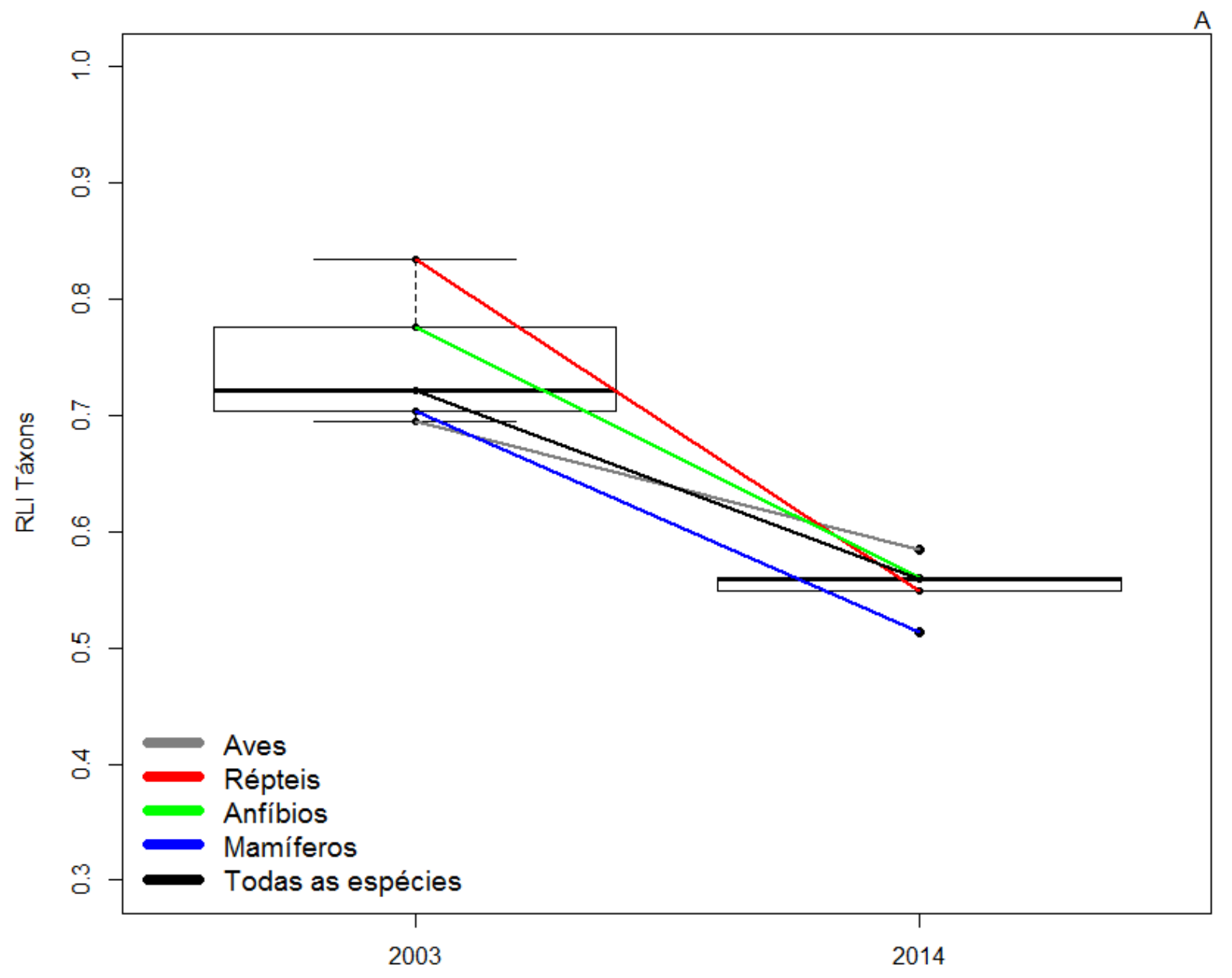



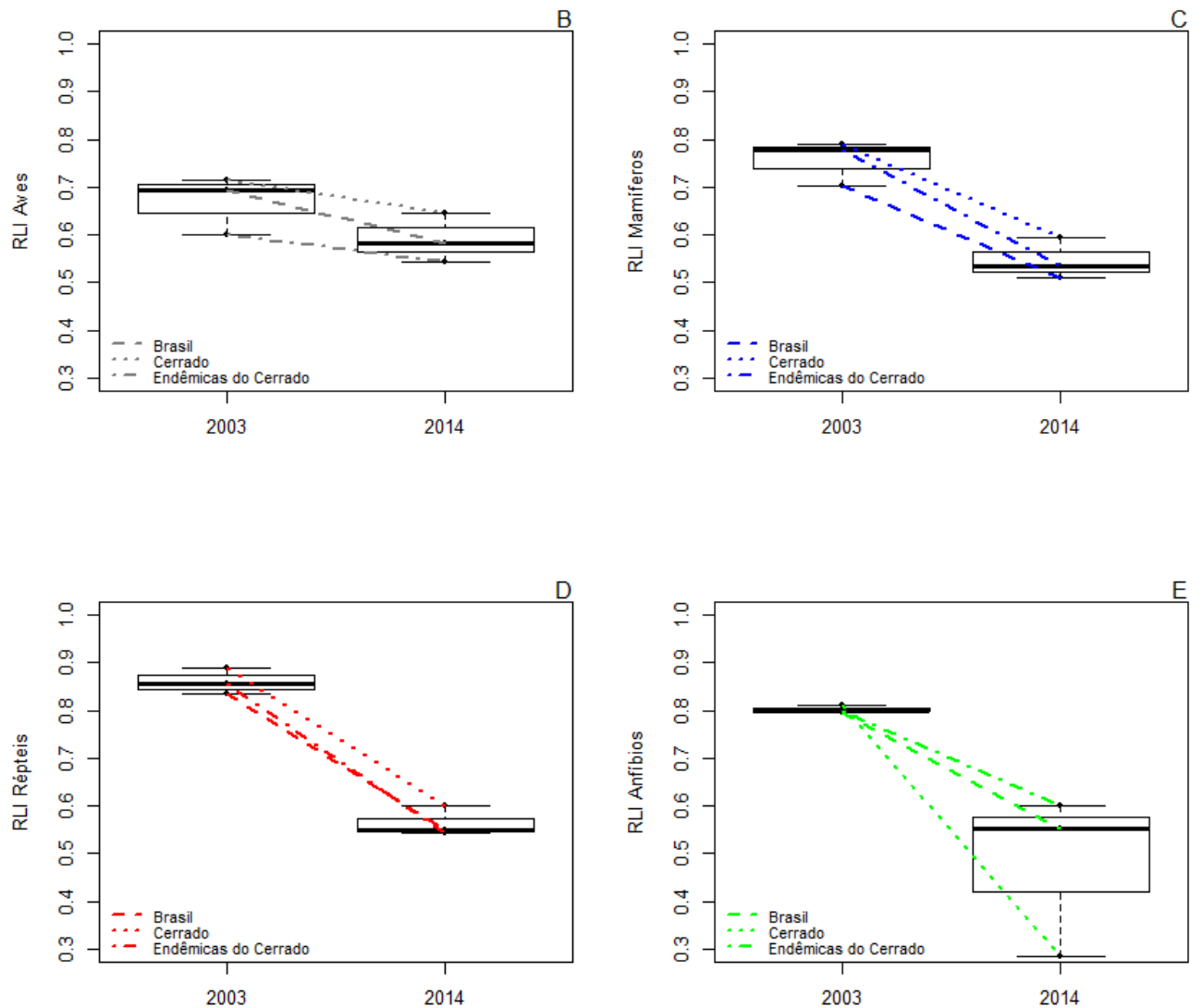

Fig. 2: Índice de espécies ameaçadas (Red List Index - RLI) entre anos de 2003 e 2014 de acordo com metodologia de Butchart et al. (2007). A - Para cada táxon e todas as espécies; B - Comparação entre as aves do Brasil, do Cerrado e endêmicas desse bioma; C - Mamíferos; D - Répteis e E - Anfíbios.

\subsection{Modelos de distribuição potencial e avaliação da perda de área das espécies.}

Para todas as espécies de aves selecionadas foram elaborados SDMs, a média de registros para treino do modelo obtida foi de 70,29 $\pm 53,48$. Para répteis foram elaborados modelos para apenas 6 espécies, a

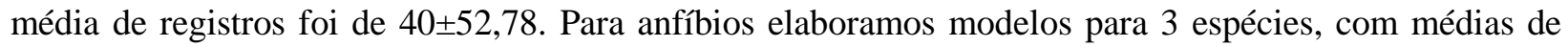
registros $13 \pm 11,27$ e utilizamos a informação de área de vida disponibilizada pela Nature Serve para Allobates goianus. Finalmente, para mamíferos, foram 5 espécies modeladas, com média de registros de 15,4ะ9,13. Incluímos algumas espécies nas análises de priorização utilizando polígonos mínimos convexos (MPC), outras espécies não foram incluídas pelos escassos registros de ocorrência. Para a maioria das espécies com mais de 5 pontos para treino, foram elaborados modelos, com desempenho considerado satisfatório pela comparação pelo teste ROC Parcial, com exceção de Kerodon acrobata, Glyphonycteris behnii e Hydrodynastes melanogigas, cujos modelos tiveram razão < 1 (Tab. S6, Fig. S3). 
A maioria das espécies endêmicas avaliada foi classificada em categorias de ameaça, por critérios de perda de hábitat, de acordo com os critérios A e B (IUCN,), de acordo com sua EOO e AOO, o que indica que o atual modelo de uso do solo afeta diretamente sua conservação (Figs 4 A e B). A maior riqueza de espécies foi observada nas porções centro-sul do bioma (Fig. 3), sobretudo no sentido sudeste. Para aves encontramos também áreas com alta sobreposição de espécies nas porções sudoeste do estado do Mato Grosso, uma das áreas com as maiores taxas de desmatamento no bioma.

A média dos SDMs das espécies-alvos será conservada a um patamar mínimo de $22 \%$ para répteis, no cenário BAU e 32\% em cenários com redução em 50\% das taxas de desmatamento (GOV.), até 2050 (Fig. S1 C e D; Tab. S7). Em 2002, répteis como, P. lativittatus já possuía um limiar abaixo de 17\% e, a partir de 2010, e B. itapetiningae e P.livida também se tornam pouco representativas, independente do cenário proposto (BAU ou GOV.). No caso dos anfíbios, P.moratoi tinha 12\% do seu SDM conservado em 2002 (Fig. S1 E e F; Tab. S7). Para aves, encontramos baixa representação para C.cyanopis, com 15\% de SDM, no cenário BAU 2010 -2050 (Fig. S1 A e B; Tab. S7). Nenhuma das espécies de mamíferos avaliados apresenta área do SDM abaixo de 17\% de proteção (Fig. S1 G e H; Tab. S7). 

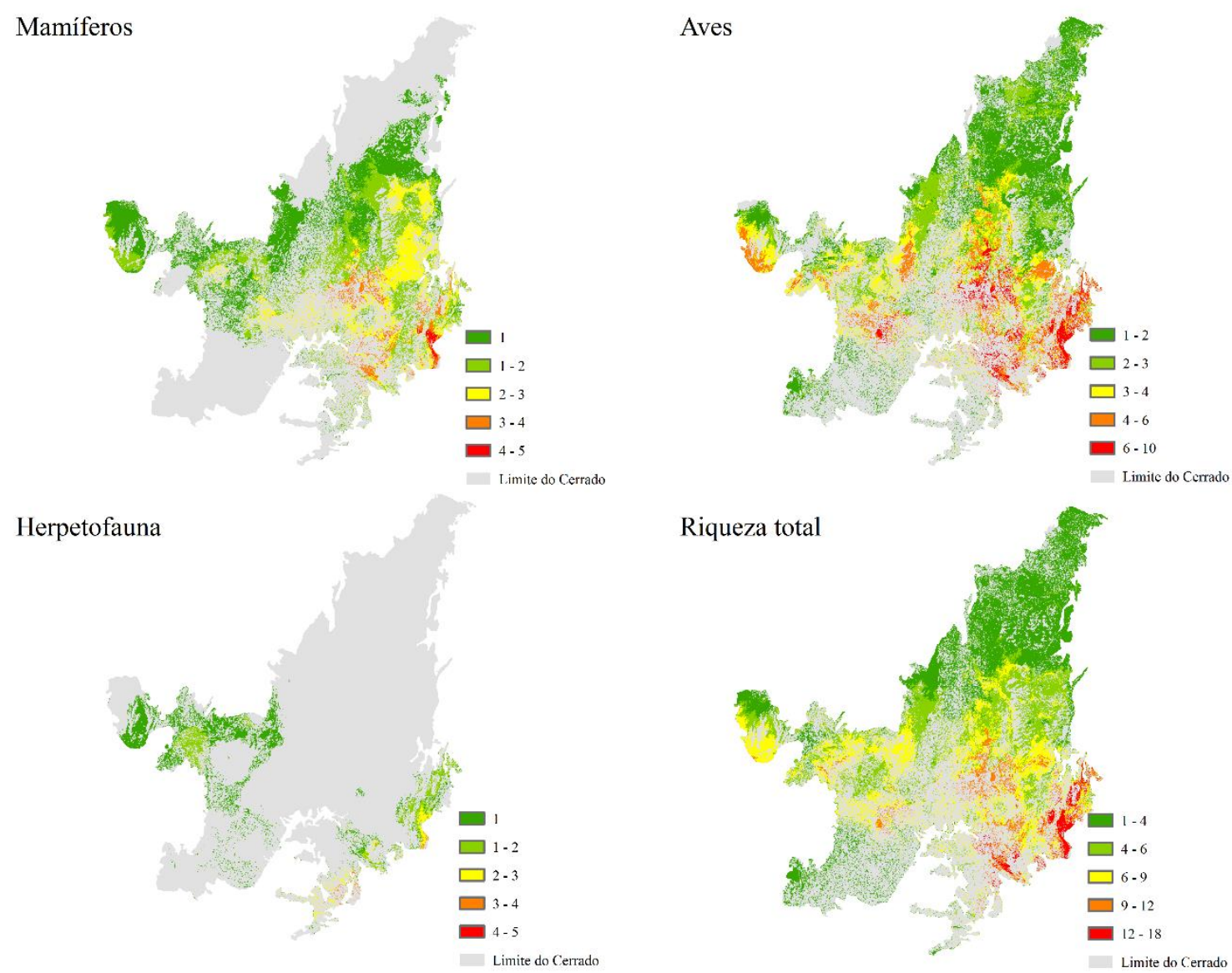

Fig. 3: Sobreposição dos SDMs das espécies-alvo, indicando a riqueza de espécies terrestre endêmicas e ameaçadas no bioma Cerrado, por táxon. 

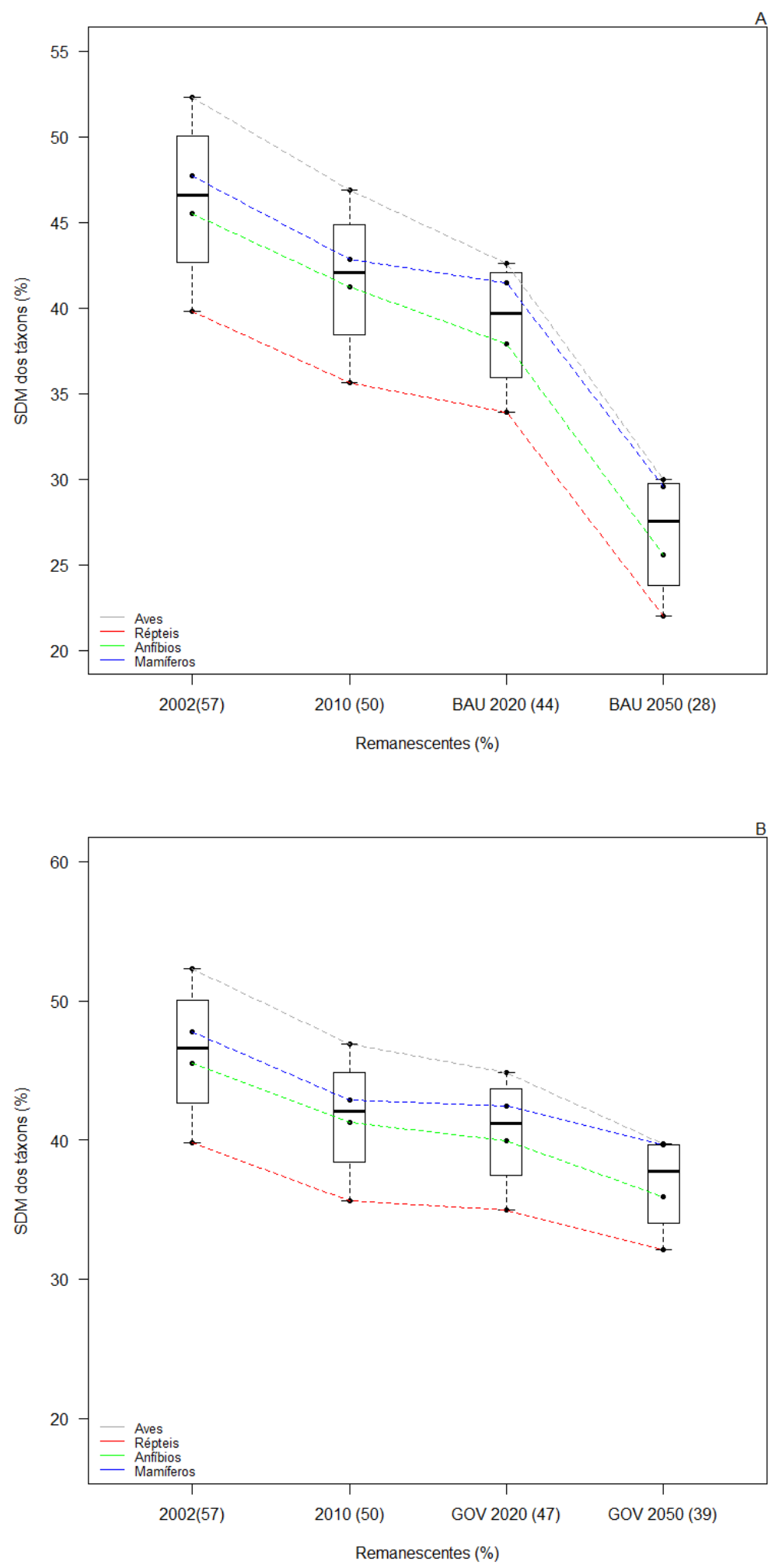

Fig. 4: Porcentagem dos SDMs dos táxons em cada um dos períodos avaliados: 2002 e 2010 (A e B) e, A -cenários BAU 2010 - 2020 e BAU 2010 - 2050. B - cenários GOV. 2010 - 2020 e GOV. 2010 - 2050 


\subsection{REDES PRIORITÁRIAS PARA A PROTEÇÃO DAS ESPÉCIES-ALVO}

A seleção de $17 \%$ das áreas com maior valor para a conservação indica que uma parte do conjunto de 41 alvos (6 espécies não foram adicionadas à solução) que representam as espécies de vertebrados endêmicas e ameaçadas do Cerrado, seriam lacunas de representação. De acordo com o cenário BAU 2010 - 2020, com 43,6\% da vegetação remanescente no Cerrado, este percentual deveria ser elevado para 23\%, para atingir a conservação de 17\% do SDM de 6 espécies-alvo (Figs 5 A, B e C). A Fig. 5A mostra a diferença na proporção de representação de cada uma das classe de vertebrados terrestres.

Dos 202.817,40 $\mathrm{Km}^{2}$ (ou 23\% necessários), 30,12\% (ou 6,91\% dos remanescentes do Cerrado) já estão representados por UCPIs. Apenas $0,25 \%$ se sobrepõe às Unidades de Conservação de Uso Sustentável (UCUs sem APAs), que não foram incluídas, à priori, nas análises. É interessante ressaltar que 10,5\% dessas áreas consideradas selecionadas se sobrepõem às Áreas de Proteção Ambiental (APAs). 62,28\% coincidem com as propostas de "Áreas Protegidas para a Conservação da Biodiversidade do Cerrado", sem considerar as UCPIs e Terras Indígenas (TIs) (MMA, 2013), e 21,54\% delas coincidem com recomendação para a criação e/ou ampliação de Unidades de Conservação. Apenas 4,27\% das áreas selecionadas coincidem com as TIs.

Quando analisamos o cenário GOV 2010 -2020 (Fig. S4) o percentual de 23\% seriam suficientes para eliminar as lacunas de conservação de 5 espécies-alvo. Para os cenários em 2050, no caso do cenário BAU, 7 espécies teriam lacunas e em GOV. 5 espécies (Fig S5).

Quando comparamos as $17 \%$ melhores áreas para a conservação, entre os cenários propostos, considerando a representação média das espécies (Fig. 6A), percebemos que não há uma forte diferenciação entre elas, já que LUCC não alterou o padrão de distribuição da riqueza das espécies, mas a disponibilidade dos hábitats nativos. Salientamos que nestes cenários a meta de proteção (17\%) foi mantida, apesar da diferença de áreas remanescentes (BAU 2010-2050, com 28,15\%; GOV 2010 -2020 e 2050, com 47\% e $39,33 \%$, respectivamente).

Nas Figs. 6C e D, é possível observar parte das áreas prioritárias suprimidas em cada um dos cenários, e quando comparamos todas as possibilidades de conversão do uso do solo, quanto a temporalidade e diminuição das taxas de desmatamento, observamos as áreas persistentes (áreas com 11,57\% dos remanescentes do Cerrado) e com maior prioridade para a conservação das espécies-alvo (Fig. 6E). 

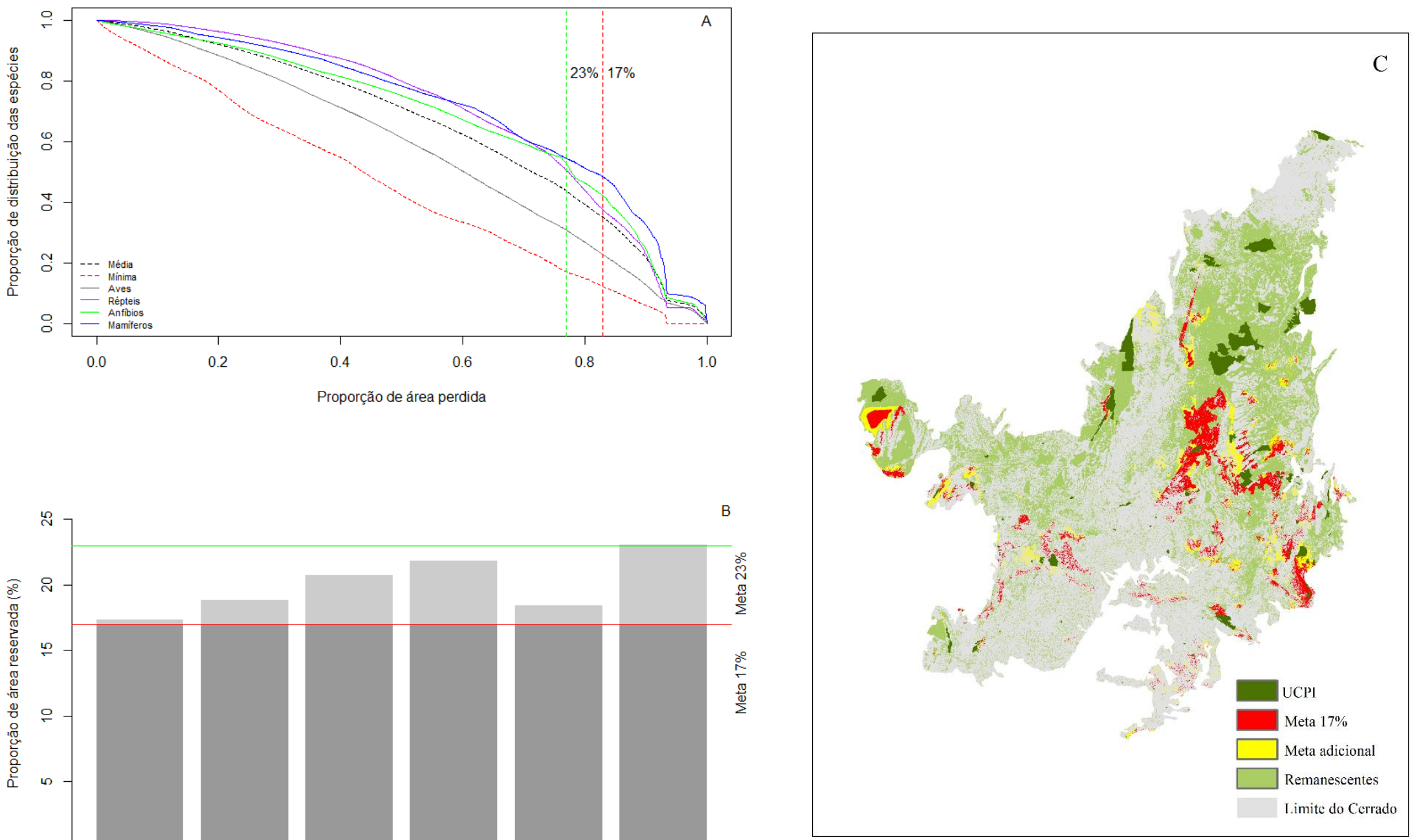

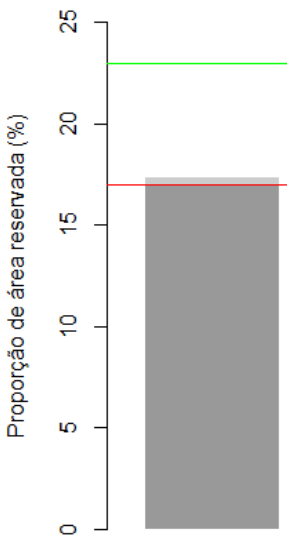

sp1

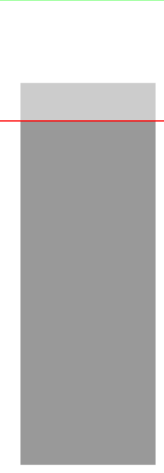

sp2

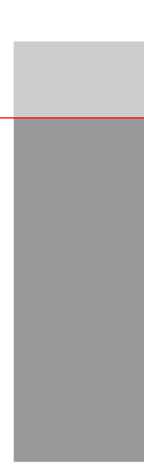

$\mathrm{sp} 3$

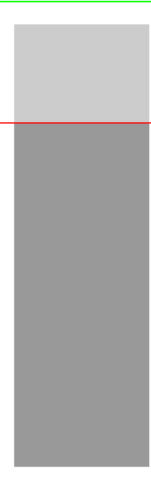

sp4

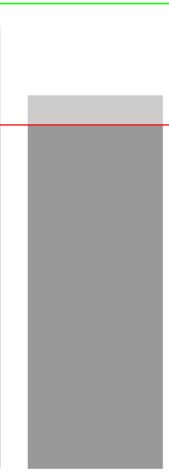

$\mathrm{sp} 5$

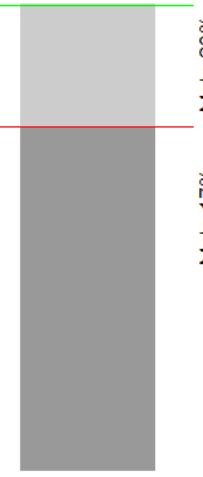

sp6

B

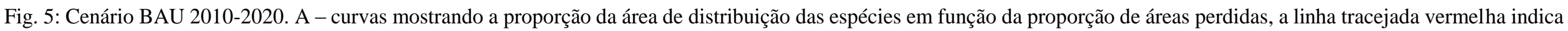

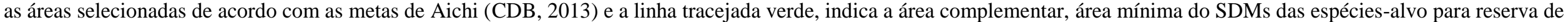

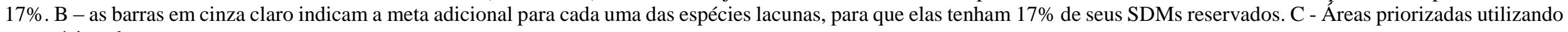
as espécies-alvo. 


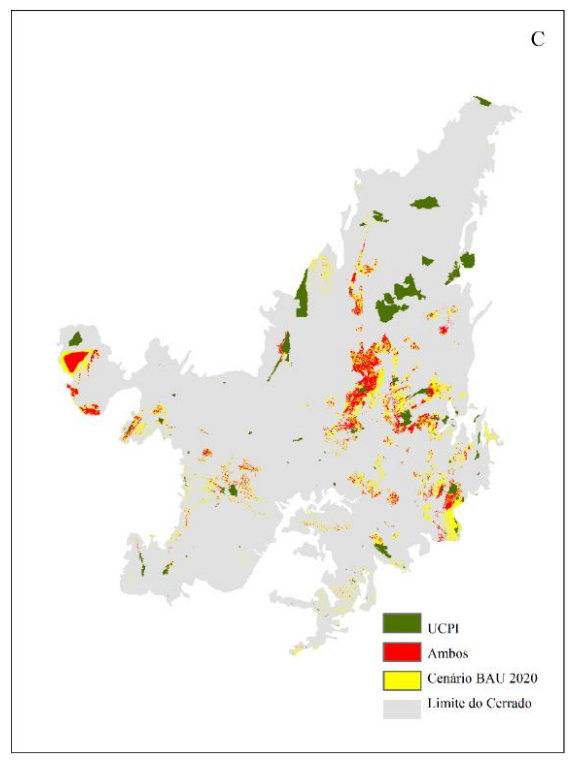

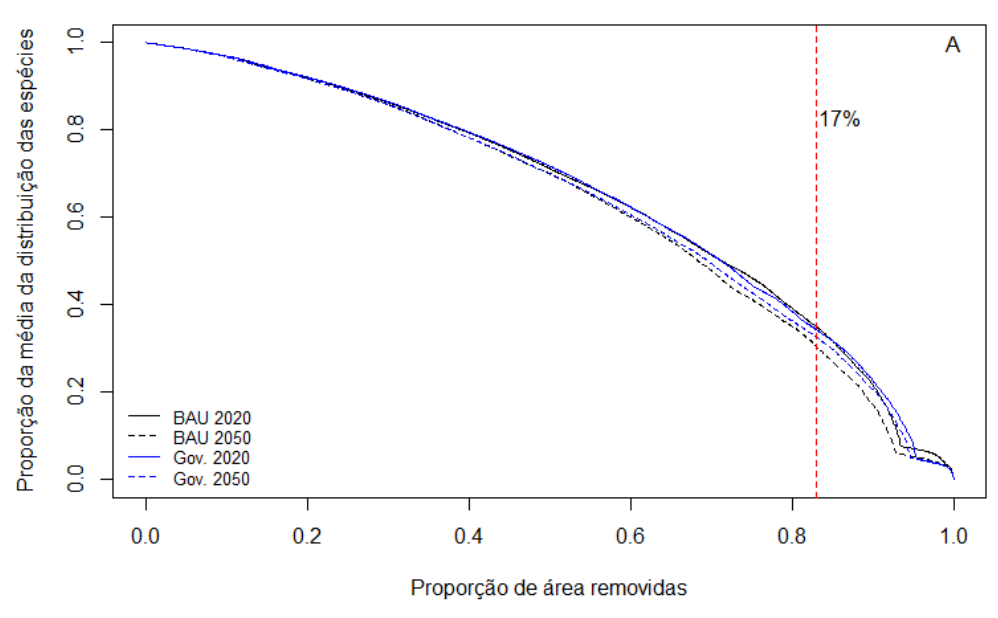

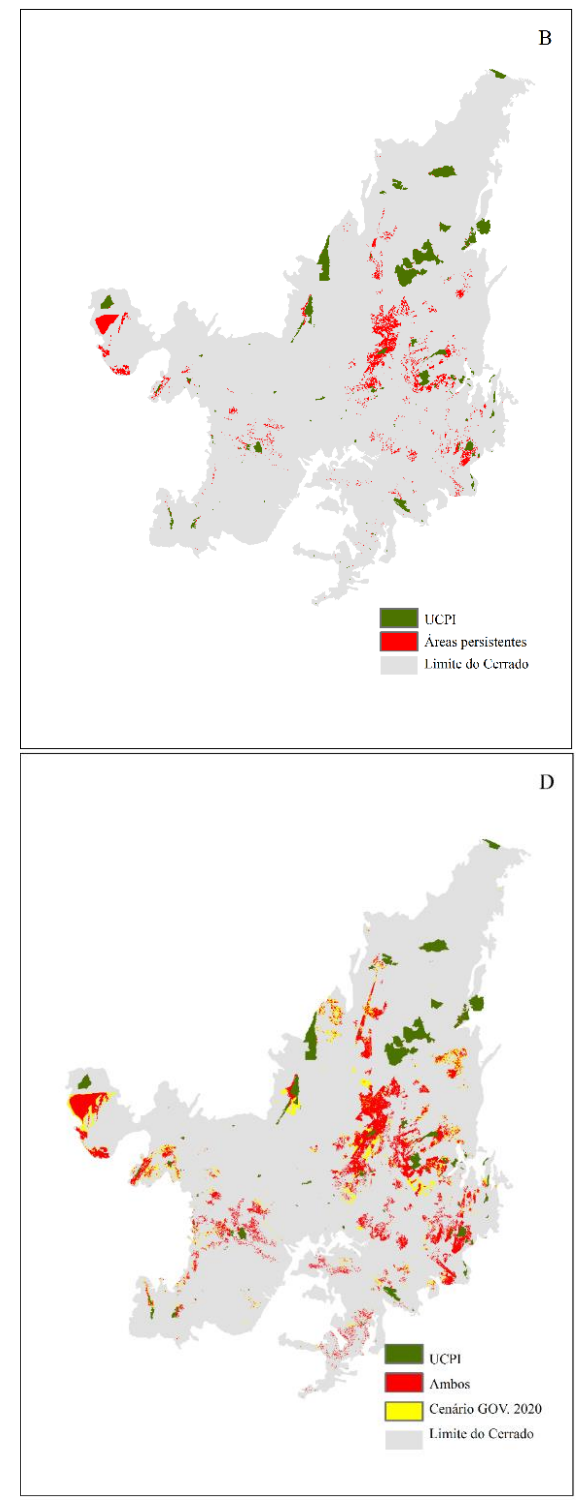

Fig. 6: A- Comparação entre todos os cenários propostos mostrando a proporção da área média de distribuição espécies em função da proporção de áreas perdidas, a linha tracejada vermelha indica as áreas selecionadas de acordo com as metas de Aichi (CDB, 2013); de 17\%. Comparação entre os cenários propostos, considerando o total de área reservada para a conservação de 17\% da área dos SDMs das espécies-alvo (C - Cenários BAU 2010-2020 e 2010-2050; e D - Cenários GOV. 2010-2020 e 2010-2050) e B Indicação das áreas pertencentes a todos os cenários propostos. 
ESTADO
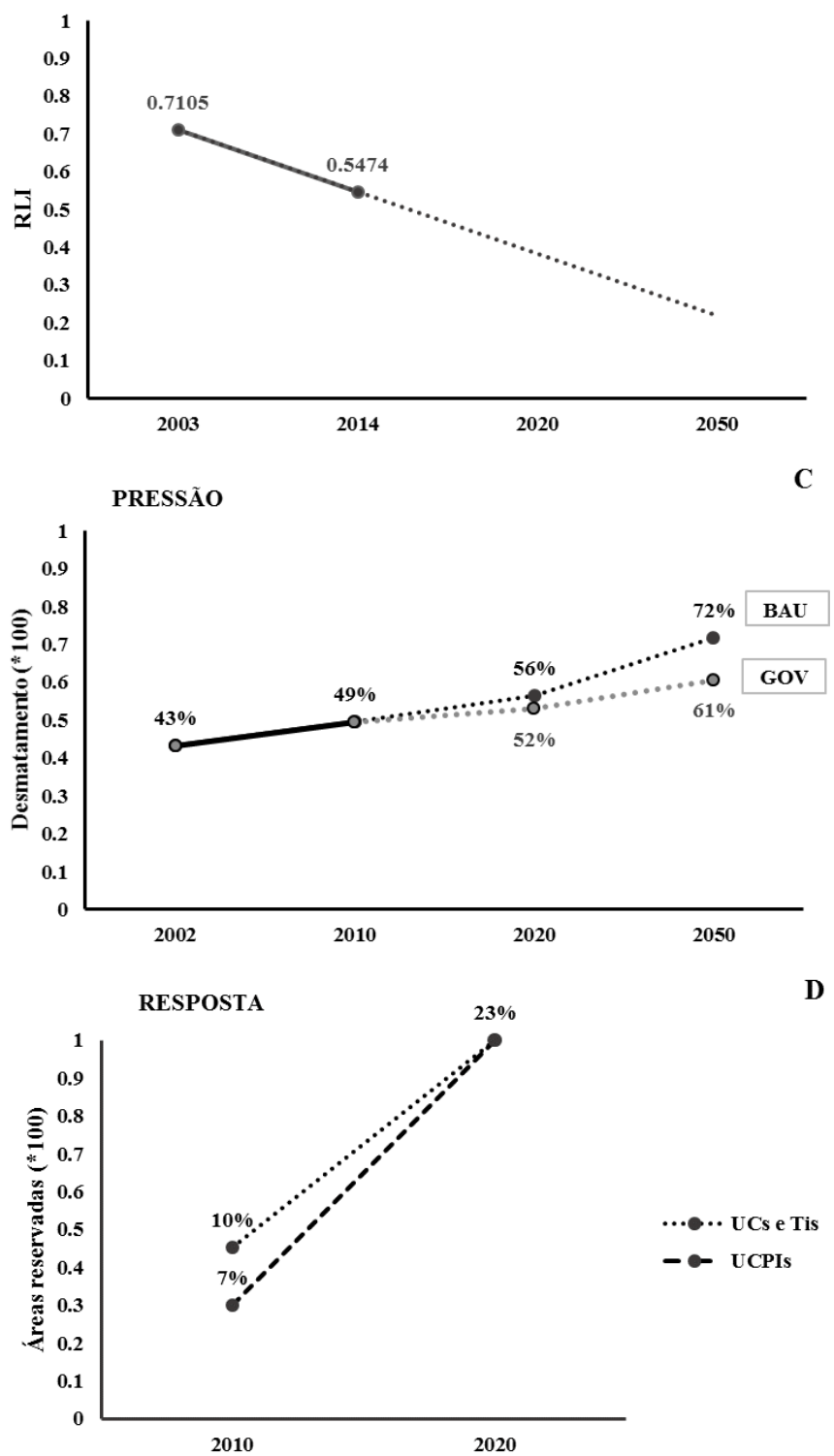

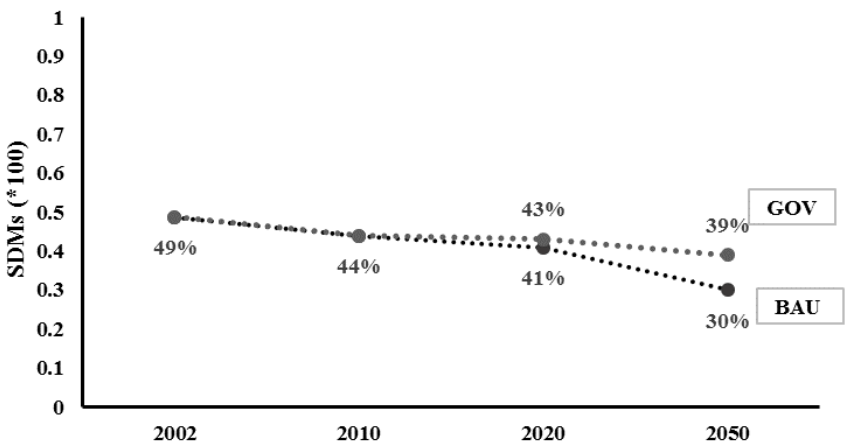

Fura 7: Resumo dos indicadores do Cerrado: Estado (A - Soma dos RLIs das espécies endêmicas e tendências futuras; B - Média dos SDMs das espécies-alvo perdidos); Pressão (C - Desmatamento nos cenários BAU e GOV) e Resposta (D - Reserva de áreas para a conservação de espécies endêmicas e ameaçadas no cenário BAU em 2020, em sobreposição com UCs e TIs e somente com as UCPIs). 


\section{DISCUSSÃO}

O RLI foi satisfatoriamente aplicado, tal como recomenda Butchart et al. (2005, 2007): as espécies foram avaliadas em 2 períodos consecutivos, a partir de delimitações distintas: grupos taxonômicos específicos, e diferentes abrangências biogeográficas. Os dados mostram que todos os táxons seguem essa tendência negativa na taxa de mudança entre as categorias de ameaça para a comparação, entre 2003 e 2014, independentemente das abrangências geográficas abordadas (Tab. S4). O índice geral caiu de 0,72 para 0,56 (em 2003 e 2014, respectivamente) preconizando uma piora no status de conservação das espécies consideradas. Assim, após 10 anos decorridos entre as duas listas consideradas, as espécies estão bem mais próximas da sua extinção. A nova lista de espécies ameaçadas de extinção brasileira, tem um grande lapso temporal da anterior e uma grande diferença em relação ao seu esforço amostral. A inclusão de espécies ameaçadas à lista poderá representar um decréscimo maior no status de conservação da biodiversidade. Pimm et al. (2014) ressaltam que muitas espécies com distribuição restrita ainda deverão ser descritas, e que a maioria delas, já estará ameaçada de extinção.

A perda de hábitats é a principal consequência da perda de biodiversidade e inferir sobre esse parâmetro requer extrapolações teóricas. Nossas análises foram baseadas em simulações: como modelos de distribuição de espécies (SDMs) e modelos de mudança na cobertura e uso da terra (LUCC). SDMs podem indicar áreas de adequabilidade à ocorrência de uma determinada espécie. Combinando estes modelos, pudemos inferir sobre a perda potencial de hábitat dessas espécies, levando em consideração a temporalidade e como a redução de 50\% no desmatamento deste bioma. Nossos alvos concentram sua riqueza de espécies no centro e no sudeste do bioma (Diniz-Filho et al., 2008), é possível que as espécies com distribuição pequena sejam quase invisíveis neste tipo de agrupamento (Pimm et al., 2014). Os répteis e os anfíbios seriam os grupos mais afetados pela redução de áreas: 100\% das espécies-alvo foram classificadas como ameaçadas pelos critérios A ou B da IUCN (2001) e, possuem áreas de distribuição pequenas em relação às aves e os mamíferos (Fig. S2). Dados globais mostram que anfíbios são os táxons mais ameaçados, seguidos dos mamíferos e répteis (Dirzo et al., 2014). Em 2050, no cenário BAU, répteis e anfíbios teriam as menores razões da área dos SDMs por área remanescente de Cerrado: 0.78 e 0.91, e sua distribuição está concentrada na região sul do bioma (Diniz-Filho et al., 2004; Mello, 2014), áreas com maiores índices de fragmentação e perda de hábitats (Barreto et al.2008). De Novaes \& Silva et al. (2014) indicam que as razões entre perda de hábitat e a perda de SDMs (de largartos) são mais drásticas em espécies de distribuição restrita.

Diante dos cenários futuros de LUCC, os planos de conservação demonstram que a reserva de áreas representativas baseado na meta de $17 \%$ seria insuficiente para a representação das espécies-alvo, e que 
este percentual deveria ser elevado ao patamar mínimo de 23\%. Se considerarmos apenas as UCPIs, como opção mais efetiva para a proteção da biodiversidade no Cerrado (ver Carranza et al., 2014; Paiva et al., 2015; Françoso et al., 2015), o Brasil ainda teria um longo caminho a percorrer para prover a proteção dessas espécies, dado que o percentual dessa categoria de UCs ainda é muito baixo neste bioma. Grande parte das áreas priorizadas se sobrepõem a UCs de Uso Sustentável, que em sua maioria (85\% de acordo Françoso et al., 2015) são APAs, categoria onde os conflitos entre uso antrópico e conservação são bastante acentuados.

Comparando todos os cenários de LUCC futuros, com e sem redução das taxas de desmatamento, uma porcentagem dessas áreas seriam as mais representativas e ao mesmo tempo aquelas com maior persistência. Mas devemos considerar que muitos outros aspectos afetam a distribuição e portanto a tenacidade das áreas reservadas para a conservação das espécies, como: as mudanças climáticas e os conflitos pelo uso do solo.

Faleiro et al. (2013) em análises de priorização no Cerrado, incluíram dimensões socioeconômicas e políticas, mostrando um grande deslocamento das prioridades espaciais, do centro-sul do bioma, para as porções mais ao norte, justamente a região de expansão da fronteira agrícola para produção de grãos formada pelos estados do Maranhão, Tocantins, Piauí e Bahia (MATOPIBA) (Gibbs et al., 2015). Diniz-Filho et al. (2008), apoiados pelos estudos de Bini et al. (2006), acreditam que o aumento de inventários de biodiversidade ao norte do bioma possam significar a expansão dos padrões de endemicidade. Nossas análises, consideraram como custos as "Áreas Prioritárias para a Conservação da Biodiversidade no Cerrado" (Brasil, 2013b), para buscar a coincidência com áreas reconhecidamente de grande importância para a biodiversidade, e encontramos grande parte dessas áreas priorizadas, coincidindo com essa nova área de expansão agrícola, corroborando Faleiro et al. (2013 e 2013b).

Além de cenários futuros de LUCC apresentados neste artigo e análises considerando os atuais conflitos pelo uso do solo (Diniz-Filho et al., 2004; Chomitz et al., 2005; Diniz-Filho et al., 2006; Diniz-Filho et al., 2006c; Silva et al., 2006; Oliveira et al., 2007; Pinto et al., 2007; Rangel et al., 2007; Diniz-Filho et al., 2008; Barreto et al., 2008; Pinto \& Grelle, 2011; Bird et al., 2012) ou considerando a conectividade dos fragmentos (Durigan et al., 2006; Durigan et al., 2009; Galetti et al., 2009; Duarte da Silva et al., 2011; Albuquerque et al, 2011), outros aspectos podem afetar o processo de planejamento da conservação. As mudanças climáticas poderão alterar a disponibilidade de hábitats e consequentemente, a distribuição das espécies, sobretudo no Cerrado (Marini et al., 2009; Kharouba et al., 2010). Introduzir essa componente de incerteza, pode significar: priorizar áreas que facilitem a dispersão das espécies no futuro (Lemes \& Loyola, 2013) ou definir um sistema de reservas que assegure as distribuições atual e futura (Loyola et al., 2013). 
Faleiro \& Loyola (2013b) associaram as alterações climáticas aos modelos preditivos de LUCC para selecionar áreas com maior estabilidade climática e ao mesmo tempo, com vegetação remanescente em cenários futuros.

Observamos alguns vieses em nossas pesquisas:

1) A utilização do RLI ainda precisa ultrapassar etapas metodológicas importantes, associadas às lacunas temporais, geográficas e taxonômicas das avaliações em países em desenvolvimento (Butchart et al., 2010), pois a metodologia ainda carrega incertezas, tais como: o uso de informações imprecisas ou incorretas para classificação das espécies; inconsistências entre avaliações (ou avaliadores); e a existência de espécies DD (Butchart et al., 2007). Este índice também reproduz os vieses das categorias das listas de espécies ameaçadas da IUCN (2001), (para detalhes sobre essa discussão ver Pimm et al., 2014). Pimm et al. (2014) alertam que as taxas de extinção se tornam mais precisas na medida do acúmulo de conhecimento sobre as espécies. Por isso, RLI deve ser complementado por outros indicadores (Butchart et al., 2007; Butchart et al., 2010) de estado, pressão e resposta (Linster, 2003), com o objetivo de medir o progresso das metas sobre biodiversidade.

2) SDMs representam a EOO e a AOO das espécies provendo superfícies contínuas a partir de informações parciais, constituindo-se em uma vantagem em relação à outros métodos (Rondinini et al., 2006, Amboni \& Laffan, 2012). Contudo, representam apenas o nicho fundamental das espécies já que, desconsideram fatores antrópicos, barreiras geográficas (que afetariam sua dispersão e colonização) e interações bióticas (Rondinini et al., 2006). Além disso, bases de dados mais consistentes podem gerar melhores modelos, mas a concentração de amostragens, ao longo de rodovias, rios, cidades, UCs, próximas à centros de pesquisa, como é o caso do Cerrado, tendem a enviesar estes resultados para sua porção centrosul (Rondinini et al., 2006; Grand et al., 2007; Carvalho et al., 2010; Diniz-Filho et al., 2008). Estas áreas melhor amostradas, são também aquelas com maiores índices de desmatamento e de fragmentação. O uso de perda de hábitat como medida da vulnerabilidade não incorpora outras ameaças, como por exemplo, a pressão pela caça ou a competição com espécies invasoras (Brooks et al., 2006).

3) O uso de espécies ameaçadas pode enviesar os planos de conservação, reservando áreas menores para a conservação e excluindo áreas de espécies não-ameaçadas das soluções apresentadas (Drummond et al., 2010). Mas áreas de espécies ameaçadas podem estar mais associadas às espécies de distribuição restrita, do que a riqueza total das espécies, indicando onde espécies podem estar ameaçadas no futuro (Pimm et al., 2014). O requerimento de área para as espécies-alvo também pode ter sido subestimados aqui, já que nossa linha de base foi uma meta política e uniforme de 17\%. As espécies podem ter diferentes requerimentos biológicos e ecológicos (Pressey et al., 2007) e que em geral, ultrapassam esse quantitativo 
(Drummond et al., 2010; De Novaes \& Silva et al., 2014). Além disso, as taxas de extinções também são afetadas pela fragmentação de hábitats (Haddad et al., 2015).

4) Neste estudo, a maioria das espécies com 5 registros de localização (para treino) gerou modelos satisfatórios, com razões de Parcial ROC >1, mas das 47 espécies alvo do estudo, somente $66 \%$ tiveram SDMs gerados, que nos permitiu analisar apenas um subconjunto das espécies ameaçadas e endêmicas desse bioma.

Nossas análises mostraram que algumas espécies-alvo precisaram de metas adicionais para sua proteção, considerando apenas o percentual acordado pela CDB. Os pequenos fragmentos ao sul do bioma, sob forte pressão antrópica, também guardam parte da representatividade do Cerrado, sobretudo para as espécies endêmicas (Diniz-Filho et al., 2008). A criação de áreas protegidas não é a panaceia para o cumprimento das metas da CDB, mas pode reduzir as taxas de extinção das espécies (Pimm et al., 2014). Seria necessário um maior esforço de coordenação dos setores governamentais e sociedade civil, para incentivar a conexão entre terras privadas protegidas legalmente, como as Reservas Legais - RLs, Áreas de Preservação Permanentes - APPs (Brasil, 2012) e Reservas Particulares do Patrimônio Natural - RPPNs (Brasil, 2000) e outras áreas protegidas, cujos objetivos centrais não são a conservação da biodiversidade, como as TIs e APAs, mas que poderiam convergir para essa finalidade. $\mathrm{O}$ atual modelo de desenvolvimento agrário para o Cerrado, principalmente para a produção de grãos e gado, precisa ser repensado, pois têm devastado a região central e avançado sobre as últimas grandes áreas remanescentes ao norte do Cerrado (ver Plano de Desenvolvimento - MATOPIBA Brasil, 2015). Pereira \& Cooper (2006) ressaltam que sem essa parcela de áreas fora das UCs, será impossível cumprir as metas da CDB.

A figura 7 resume os indicadores tratados neste capítulo: a deterioração do status de conservação das espécies (Fig. 7A e B); a crescente perda de hábitats pelo desmatamento do Cerrado (Fig. 7C) e, a necessidade de complementação do sistema de áreas protegidas e/ou a efetivação de políticas que garantam sua conectividade com áreas focais para a conservação da biodiversidade (Fig. 7D). 


\section{CONCLUSÃO}

O desenvolvimento do território avança sobre os espaços naturais, promovendo a crescente perda de hábitats das espécies. Os modelos de LUCC buscam projetar os padrões do passado simulando o futuro, e propondo cenários prospectivos que podem auxiliar nas tomadas de decisões. Nossos estudos demostraram que o uso de modelos espacialmente explícitos podem ser importantes ferramentas para reavaliação dos objetivos da conservação. Aderir às metas propostas pela $\mathrm{CDB}$ exige dos países um monitoramento periódico sobre as pressões, os estados de conservação e quais respostas o país vem produzindo para equacionar seus problemas (De Heer et al., 2005; Butchart et al., 2012; Han et al., 2014). Por isso, o desenvolvimento dos indicadores de meio ambiente, à exemplo de outros indicadores setoriais, são tão importantes, pois mostram tendências e permitem comparações entre abrangências geográficas distintas. $\mathrm{O}$ planejamento da conservação cada vez mais se mune dessas ferramentas para resguardar possíveis lacunas de conservação antes que esses remanescentes sejam perdidos. Assim, utilizando como alvos espécies endêmicas e ameaçadas do Cerrado, pudemos demonstrar que a transformação de $17 \%$ das áreas nativas do Cerrado em unidades de conservação seria insuficiente para resguardar suas lacunas de conservação em cenários futuros. Para alcançar a meta proposta em Aichi, o percentual de proteção do Cerrado deveria ser elevado para $23 \%$ em áreas estratégicas e de alta importância biológica. Os grandes remanescentes no centro e ao norte do bioma ajudam a proteger uma porção representativa dessas espécies, mas será necessário complementar a proteção com outras áreas ao sul do bioma, que concentram a maior parte dessa riqueza, mas que também têm altos índices de fragmentação e pressão antrópica. 


\section{REFERÊNCIAS BIBLIOGRAFICAS}

Albuquerque, F. S. De, Assunção-Albuquerque, M. J. T., Gálvez-Bravo, L., Cayuela, L., Rueda, M., \& Benayas, J. M. R. (2011). Identification of Critical Areas for Mammal Conservation in the Brazilian Atlantic Forest Biosphere Reserve. Natureza \& Conservação, 9(1), 73-78. http://doi.org/10.4322/natcon.2011.009

Amboni, M.P.M., Laffan, S.W., 2013. The effect of species geographical distribution estimation methods on richness and phylogenetic diversity estimates. International Journal of Geographical Information Science, 37-41.

Araújo, M. B. \& Williams, P. H. (2000). Selecting areas for species persistence using occurrence data. Biological Conservation, 96(3), 331-345. http://doi.org/10.1016/S0006-3207(00)00074-4

Barreto, B. D. S., Oliveira, G. De, Pinto, M. P., Mauricio, L., Alexandre, J., Diniz, F., \& Blamires, D. (2008). Riqueza de espécies de emberizídeos e conflitos de conservação no Cerrado brasileiro. Acta Sci.Biol.Sci., 67-72.

Barve, N. (2008), Tool for Partial-ROC (Biodiversity Institute, Lawrence, KS), ver 1.0.

Bini, L. M., Diniz-Filho, J. A. F., Rangel, T. F. L. V. B., Bastos, R. P., \& Pinto, M. P. (2006). Challenging Wallacean and Linnean shortfalls: knowledge gradients and conservation planning in a biodiversity hotspot. Diversity and Distributions, 12(5), 475-482. http://doi.org/10.1111/j.13669516.2006.00286.x

Bird, J. P., Buchanan, G. M., Lees, A. C., Clay, R. P., Develey, P. F., Yépez, I., \& Butchart, S. H. M. (2012). Integrating spatially explicit habitat projections into extinction risk assessments: a reassessment of Amazonian avifauna incorporating projected deforestation. Diversity and Distributions, 18(3), 273281. http://doi.org/10.1111/j.1472-4642.2011.00843.x

Bonvicino, C. R., Lindbergh, S. M., Faria, M. B., \& Bezerra, A. M. R. (2012). The eastern boundary of the brazilian cerrado: A hotspot region. Zoological Studies, 51(7), 1207-1218.

Brandão, A. S. P., Rezende, G. C. De, \& Marques, R. W. D. C. (2006). Crescimento agrícola no período 1999/2004: a explosão da soja e da pecuária bovina e seu impacto sobre o meio ambiente. Economia Aplicada, 10(2), 249-266. Retrieved from http://www.scielo.br/scielo.php?script=sci_pdf\&pid=S1413-

$80502006000200006 \& \operatorname{lng}=\mathrm{en} \& \mathrm{nrm}=\mathrm{iso} \& \mathrm{tlng}=\mathrm{pt}$

Brannstrom, C., Jepson, W., Filippi, A. M., Redo, D., Xu, Z., \& Ganesh, S. (2008). Land change in the Brazilian Savanna (Cerrado), 1986-2002: Comparative analysis and implications for land-use policy. Land Use Policy, 25(4), 579-595. http://doi.org/10.1016/j.landusepol.2007.11.008

Brasil (1994). Decreto Legislativo n ${ }^{\circ} 2$, de 3/02/1994

Brasil (1998). Decreto Legislativo n ${ }^{\circ} 2.519,16 / 03 / 1998$

Brasil (2000). Lei $\mathrm{n}^{\text {o. }}$ 9.985, de 18/07/2000 
Brasil (2002). Decreto $\mathrm{n}^{\circ}$ 4.340, de 22 de Agosto de 2002.

Brasil (2003). Instrução Normativa ${ }^{0 .}$ 03, de 26 de Maio de 2003

Brasil (2004). Mapa de Biomas do Brasil. Escala 1.5.000.000. Instituto Brasileiro de Geografia e Estatística - IBGE, Rio de Janeiro.

Brasil (2006). Resolução CONABIO n³, de 21 de Dezembro de 2006

Brasil (2011). Sistema de Informações Compartilhadas (SISCOM). Acessado em 20.08.2011. http://siscom.ibama.gov.br/monitora_biomas/

Brasil (2012). Lei $\mathrm{n}^{\text {o. }} 12.651$, de 25/05/2012

Brasil (2013). Resolução CONABIO nº 6, de 3 de Setembro de 2013

Brasil (2013b). Base digital de dados geoespacializados das áreas prioriárias do Cerrado, dados nãopublicados.

Brasil (2014). Portaria ${ }^{\circ}$ 444, de 7 de Dezembro de 2014.

Brasil (2015). Decreto no 8.447, de 6 de Maio de 2015

Brooks, T. M., Mittermeier, R. a., da Fonseca, G. a. B., Gerlach, J., Hoffmann, M., Lamoreux, J. F., Rodrigues, a S. L. (2006). Global Biodiversity Conservation Priorities. Science, 313(July), 58-61. http://doi.org/10.1126/science.1127609

Butchart, S. H. M., Stattersfield, a J., Baillie, J., Bennun, L. a, Stuart, S. N., Akçakaya, H. R., ... Mace, G. M. (2005). Using Red List Indices to measure progress towards the 2010 target and beyond. Philosophical Transactions of the Royal Society of London. Series B, Biological Sciences, 360(1454), 255-68. http://doi.org/10.1098/rstb.2004.1583

Butchart, S. H. M., Akçakaya, H. R., Chanson, J., Baillie, J. E. M., Collen, B., Quader, S., Hilton-Taylor, C. (2007). Improvements to the Red List Index. PLoS ONE, 2(1). http://doi.org/10.1371/journal.pone.0000140

Butchart, S. H. M., Walpole, M., Collen, B., van Strien, a., Scharlemann, J. P. W., Almond, R. E. a., Watson, R. (2010). Global Biodiversity: Indicators of Recent Declines. Science, 328(5982), 1164-1168. http://doi.org/10.1126/science.1187512

Butchart, S. H. M., Scharlemann, J. P. W., Evans, M. I., Quader, S., Aricó, S., Arinaitwe, J., ... Woodley, S. (2012). Protecting important sites for biodiversity contributes to meeting global conservation targets. PLoS ONE, 7(3), 1-10. http://doi.org/10.1371/journal.pone.0032529

Cabeza, M., \& Moilanen, A. (2001). Design of reserve networks and the persistence of biodiversity. Trends in Ecology \& Evolution, 16(5), 242-248.

Carranza, T., Balmford, A., Kapos, V., \& Manica, A. (2014). Protected area effectiveness in reducing conversion in a rapidly vanishing ecosystem: The Brazilian Cerrado. Conservation Letters, 7(3), 216223. http://doi.org/10.1111/conl.12049 
Carvalho, S. B., Brito, J. C., Pressey, R. L., Crespo, E., \& Possingham, H. P. (2010). Simulating the effects of using different types of species distribution data in reserve selection. Biological Conservation, 143(2), 426-438. http://doi.org/10.1016/j.biocon.2009.11.010

Chomitz, K. M., Alger, K., Thomas, T. S., Orlando, H., \& Nova, P. V. (2005). Opportunity costs of conservation in a biodiversity hotspot: the case of southern Bahia. Environment and Development Economics, 10(3), 293-312. http://doi.org/10.1017/S1355770X05002081

Clark Labs. (2009). Land change modeler. Acessado em 12/03/2014. https://clarklabs.org/category/whitepapers/

CBD-Convention of Biological Diversity (1992). United Nations. Concluded on 5 June 1992. Rio de Janeiro, Brazil.

Colli, G. R., Bastos, R. P. \& Araújo, A. B. in The Cerrados of Brazil - Ecology and Natural History of a Neotropical Savanna (eds P.S. Oliveira \& R.J. Marquis) 223-241 (Columbia University Press, 2002).

Cowling, R. M., Pressey, R. L., Lombard, A. T., Desmet, P. G. \& Ellis, A. G. (1999). From representation to persistence: requirements for a sustainable system of conservation areas in the species rich Mediterranean-climate desert of southern Africa. Biodiversity and Distributions, 5, 51-71.

De Heer, M., Kapos, V., \& ten Brink, B. J. E. (2005). Biodiversity trends in Europe: development and testing of a species trend indicator for evaluating progress towards the 2010 target. Philosophical Transactions of the Royal Society of London. Series B, Biological Sciences, 360(1454), 297-308. http://doi.org/10.1098/rstb.2004.1587

De Novaes e Silva, V., Pressey, R. L., Machado, R. B., VanDerWal, J., Wiederhecker, H. C., Werneck, F. P., \& Colli, G. R. (2014). Formulating conservation targets for a gap analysis of endemic lizards in a biodiversity hotspot. Biological Conservation, $180, \quad 10$. http://doi.org/10.1016/j.biocon.2014.09.016

Diniz-Filho, J. A. F., Bini, L. M., Vieira, C. M., Souza, M. C. De, Bastos, R. P., Brandão, D., \& Oliveira, L. G. (2004). Spatial patterns in species richness and priority areas for conservation of anurans in the Cerrado region, Central Brazil. Amphibia-Reptilia, 63-75.

Diniz-Filho, J. A. F., Bini, L. M., Pinto, M. P., Rangel, T. F. L. V. B., Carvalho, P., \& Bastos, R. P. (2006). Anuran species richness, complementarity and conservation conflicts in Brazilian Cerrado. Acta Oecologica, 29(1), 9-15. http://doi.org/10.1016/j.actao.2005.07.004

Diniz-Filho, J. A. F., Bini, L. M., Pinto, M. P., Terribile, L. C., de Oliveira, G., Vieira, C. M., ... Bastos, R. P. (2008). Conservation planning: a macroecological approach using the endemic terrestrial vertebrates of the Brazilian Cerrado. Oryx, 42(04), 567. http://doi.org/10.1017/S0030605308001129

Dirzo, R., Young, H. S., Galetti, M., Ceballos, G., Isaac, N. J. B., \& Collen, B. (2014). Defaunation in the Anthropocene. Science, 345(6195), 401-406. http://doi.org/10.1126/science.1251817

Drummond, S. P., Wilson, K. a., Meijaard, E., Watts, M., Dennis, R., Christy, L., \& Possingham, H. P. (2010). Influencia del enfoque en una especie amenazada sobre la planificación de la conservación. Conservation Biology, 24(2), 441-449. http://doi.org/10.1111/j.1523-1739.2009.01346.x 
Duarte da Silva, M.S.; Smith, O.P; Araújo Teixeira, R. (2011). Modelagem matemárica para seleção de áreas prioritárias à conservacão ou restauração no Cerrado Goiano. Mercator - Revista de Geografia Da UFC, 10(Set-Dez, n.23), 225-236. Retrieved from http://www.redalyc.org/src/inicio/ArtPdfRed.jsp?iCve=273621468017

Durigan, G., Ivanauskas, N. M., Nalon, M. A., Ribeiro, M. C., Kanashiro, M. M., Costa, H. B., \& Santiago, C. D. M. (2009). Protocolo de avaliação de áreas prioritárias para a conservação da Mata Atlântica na região da Serra do Mar/Paranapiacaba. Rev.Inst.Flor., 39-54.

Durigan, G.; Siqueira, M.F.; Franco, G.A.D.C.; Ratter, J. A. (2006). Seleção de fragmentos prioritários para a criação de unidades de conservação do Cerrado no Estado de São Paulo. Rev. Inst. Flor., (1), 2337.

ESRI (2015). ArcGis Desktop: Release 10.3.1. Redlands, CA: Environmental Systems Research Institute.

Fahrig, L. (2001). How much habitat is enough? Biological Conservation, 100(1), 65-74. http://doi.org/10.1016/S0006-3207(00)00208-1

Fairbanks, D. (2001). Species and environment representation: selecting reserves for the retention of avian diversity in KwaZulu-Natal, South Africa. Biological Conservation, 98(3), 365-379. http://doi.org/10.1016/S0006-3207(00)00179-8

Faleiro, F. V., \& Loyola, R. D. (2013). Socioeconomic and political trade-offs in biodiversity conservation: a case study of the Cerrado Biodiversity Hotspot, Brazil. Diversity and Distributions, 19(8), 977-987. http://doi.org/10.1111/ddi.12072

Faleiro, F. V., Machado, R. B., \& Loyola, R. D. (2013b). Defining spatial conservation priorities in the face of land-use and climate change. Biological Conservation, 158, 248-257. http://doi.org/10.1016/j.biocon.2012.09.020

Françoso, R. D., Brandão, R., Nogueira, C. C., Salmona, Y. B., Machado, R. B., \& Colli, G. R. (2015). Habitat loss and the effectiveness of protected areas in the Cerrado Biodiversity Hotspot. Natureza $E$ Conservação, 13(1), 35-40. http://doi.org/10.1016/j.ncon.2015.04.001

Galetti, M., Giacomini, H. C., Bueno, R. S., Bernardo, C. S. S., Marques, R. M., Bovendorp, R. S., Peres, C. a. (2009). Priority areas for the conservation of Atlantic forest large mammals. Biological Conservation, 142(6), 1229-1241. http://doi.org/10.1016/j.biocon.2009.01.023

GBIF (2015). Acessado em 23/09/2015. http://www.gbif.org/

Gibbs, B. H. K., Rausch, L., Munger, J., Schelly, I., Morton, D. C., Noojipady, P., Cerrado, E. (2015). Brazil's Soy Moratorium. Science, 347(6220), 377-378. http://doi.org/10.1126/science.aaa0181

Grand, J., Cummings, M. P., Rebelo, T. G., Ricketts, T. H., \& Neel, M. C. (2007). Biased data reduce efficiency and effectiveness of conservation reserve networks. Ecology Letters, 10(5), 364-74. http://doi.org/10.1111/j.1461-0248.2007.01025.x 
Haddad, N. M., Brudvig, L. a., Clobert, J., Davies, K. F., Gonzalez, a., Holt, R. D., ... Townshend, J. R. (2015). Habitat fragmentation and its lasting impact on Earth's ecosystems. Science Advances, 1(2), e1500052-e1500052. http://doi.org/10.1126/sciadv.1500052

Hijmans, R.J., Cameron S.E., Parra J.L., Jones P.G. \& Jarvis A. (2005). Very high resolution interpolated climate surfaces for global land areas. International Journal of Climatology, 25: 1965-1978.

ICMBio (2014). Acessado em 01/02/2016. http://www.icmbio.gov.br/portal/biodiversidade/faunabrasileira/lista-de-especies-dados-insuficientes.html

IUCN (2001). IUCN Red List Categories and Criteria - version 3.1. Gland, Swizerland: IUCN - The World Conservation Union. 30p.

Klink, C. A, \& Machado, R. B. (2005). Conservation of the Brazilian Cerrado. Conservation Biology, 19(3), 707-713. http://doi.org/10.1111/j.1523-1739.2005.00702.x

Kharouba, H. M., \& Kerr, J. T. (2010). Just passing through: Global change and the conservation of biodiversity in protected areas. Biological Conservation, 143(5), 1094-1101. http://doi.org/10.1016/j.biocon.2010.02.002

Leal, S.B. E; Silva, D.Q.; Ramalho, D. de F.; Miller, B.G.; Passos Filhos, P. de B.; Prado Neto, J.G.; Guerra, D. de Q.; Moura, G.J.B de; Lyra-Neves, R.M. de; Telino-Júnior, W. R. (2013). Extension of the geographical distribution of Lonchophylla dekeyseri Taddei, Vizotto and Sazima, 1983 (Chiroptera: Phyllostomidae): New record in northeastern Brazil. Chiroptera Neotropical, 19(2), 1220-1225. Retrieved from https://chiroptera.unb.br/index.php/cn/article/view/200

Leathwick, J., Moilanen, A., Francis, M., Elith, J., Taylor, P., Julian, K., Duffy, C. (2008). Novel methods for the design and evaluation of marine protected areas in offshore waters. Conservation Letters, 1(2), 91-102. http://doi.org/10.1111/j.1755-263X.2008.00012.x

Lemes, P., \& Loyola, R. D. (2013). Accommodating Species Climate-Forced Dispersal and Uncertainties in Spatial Conservation Planning. PLoS ONE, 8(1), e54323. http://doi.org/10.1371/journal.pone.0054323

Lima, A.P.; Caldwell, J.P. \& Strussmann, C. (2009). Redscription of Allobates brunneus (Cope) 1887 (Anura: Aromobatidae: Allobatinae), with a description of the tadpole, call, and reproductive behavior. Zootaxa, 16, 1-16.

Linster, M. (OECD). (2003). OECD Environmental Indicators: development, measurement and use. OECD (Vol. 25).

Lopes, L. E. (2009). Taxonomia, Biogeografia e Conservação das aves endêmicas do Cerrado. UFMG, Tese de Doutorado, 261p.

Loyola, R. D., Lemes, P., Nabout, J. C., Trindade-Filho, J., Sagnori, M. D., Dobrovolski, R., \& Diniz-Filho, J. A. F. (2013). A straightforward conceptual approach for evaluating spatial conservation priorities under climate change. Biodiversity and Conservation, 22(2), 483-495. http://doi.org/10.1007/s10531012-0424-x 
Machado, A.B.M., Martins, C.S., Drummond, G.M. (eds) (2005). Lista da fauna brasileira ameaçada de extinção: incluindo a Lista das espécies quase ameaçadas e deficientes em dados. Belo Horizonte, Biodiversitas. 157p.

Margules, C. R., \& Pressey, R. L. (2000). Systematic conservation planning. Nature, 405(6783), $243-53$. http://doi.org/10.1038/35012251

Marini, Â. M., Barbet-massin, M., Esteves, L., \& Jiguet, F. (2009). Major current and future gaps of Brazilian reserves to protect Neotropical savanna birds. Biological Conservation, 142(12), 30393050. http://doi.org/10.1016/j.biocon.2009.08.002

Mello, P. L. H. De. (2014). Répteis Squamata endêmicos do Cerrado: Perdas de hábitat e conservação em cenários futuros. Dissertação, Universidade de Brasília.

Moilanen, A. (2005). Methods for reserve selection: Interior point search. Biological Conservation, 124(4), 485-492. http://doi.org/10.1016/j.biocon.2005.02.012

Moilanen, A. (2007). Landscape zonation, benefit functions and target-based planning: Unifying reserve selection strategies. Biological Conservation, 134(4), 571-579. http://doi.org/10.1016/j.biocon.2006.09.008

Myers, N., Mittermeier, R. a, Mittermeier, C. G., da Fonseca, G. a, \& Kent, J. (2000). Biodiversity hotspots for conservation priorities. Nature, 403(6772), 853-8. http://doi.org/10.1038/35002501

Nyári, Á. S., \& Reddy, S. (2013). Comparative Phyloclimatic Analysis and Evolution of Ecological Niches in the Scimitar Babblers (Aves: Timaliidae: Pomatorhinus). PLoS ONE, 8(2). http://doi.org/10.1371/journal.pone.0055629

Nogueira, C., Ribeiro, S., Costa, G. C., \& Colli, G. R. (2011). Vicariance and endemism in a Neotropical savanna hotspot: Distribution patterns of Cerrado squamate reptiles. Journal of Biogeography, 38(10), 1907-1922. http://doi.org/10.1111/j.1365-2699.2011.02538.x

Noss, R. F. (1990). Indicators for Monitoring Biodiversity: A Hierarchical Approach. Conservation Biology, 4(4), 355-364.

Oliveira, G.dr;Barreto, B de S.; Pinto, M.P.; Diniz-Filho, J.A.F.; Blamires, D. (2007). Padrões espaciais de diversidade da Família Emberizidae ( Aves: Passeriformes ) e seleção de áreas prioritárias para conservação no Cerrado. Lundiana 8, 97-106.

Paglia, A. P., Rylands, A. B., Herrmann, G., Aguiar, L. M. S., Chiarello, A. G., Leite, Y. L. R., ... Siciliano, S. (2012). Lista Anotada dos Mamíferos do Brasil. Conservation Biology, (6).

Paiva, R. J. O., Brites, R. S., \& Machado, R. B. (2015). The role of protected areas in the avoidance of anthropogenic conversion in a high pressure region: A matching method analysis in the core region of the brazilian cerrado. PLoS ONE, 10(7), 1-17. http://doi.org/10.1371/journal.pone.0132582

Pereira, H. M., \& David Cooper, H. (2006). Towards the global monitoring of biodiversity change. Trends in Ecology and Evolution, 21(3), 123-129. http://doi.org/10.1016/j.tree.2005.10.015 
Pereira, H. M., Leadley, P. W., Proença, V., Alkemade, R., Scharlemann, J. P. W., Fernandez-Manjarrés, J. F., ... Walpole, M. (2010). Scenarios for global biodiversity in the 21st century. Science, 330(6010), 1496-1501. http://doi.org/10.1126/science.1196624

Pérez-Vega, A., Mas, J. F., \& Ligmann-Zielinska, A. (2012). Comparing two approaches to land use/cover change modeling and their implications for the assessment of biodiversity loss in a deciduous tropical forest. Environmental Modelling and Software, 29(1), 11-23. http://doi.org/10.1016/j.envsoft.2011.09.011

Peterson, a. T., Papeş, M., \& Soberón, J. (2008). Rethinking receiver operating characteristic analysis applications in ecological niche modeling. Ecological Modelling, 213(1), 63-72. http://doi.org/10.1016/j.ecolmodel.2007.11.008

Phillips, S.J., Anderson, R.P., Schapire, R.E. (2006). Maximum entropy modeling of species geographic distributions. Ecological Modelling 190, 231-259.

Pimm, S. L., Jenkins, C. N., Abell, R., Brooks, T. M., Gittleman, J. L., Joppa, L. N., ... Sexton, J. O. (2014). The biodiversity of species and their rates of extinction, distribution, and protection. Science (New York, N.Y.), 344(6187), 1246752. http://doi.org/10.1126/science.1246752

Pinto, M.P., Mathias, P.V.C., Blamires, D., Diniz-Filho, J.A.F., Bini, L.M., 2007. Selecting priority areas to conserve Psittacines in the Brazilian cerrado: minimizing human-conservation conflicts. Bird Conserv. Int. 17, 13. doi:10.1017/S0959270906000578

Pinto, M. P., \& Grelle, C. E. V. (2011). Minimizing conservation conflict for endemic primate species in Atlantic forest and uncovering knowledge bias. Environmental Conservation, 39(01), 30-37. http://doi.org/10.1017/S0376892911000440

Prates, A.P.L.\& Irving, M. d. A. (2015). Conservação da Biodiversidade e Políticas Públicas para as Áreas Protegidas no Brasil: Desafios e Tendências da Origem da CDB às Metas de Aichi. Revista Brasileira de Políticas Püblicas, 1, 28-58. http://doi.org/10.1017/CBO9781107415324.004

Pressey, R.L.;Taffs, K. H. (2001). Scheduling priority conservation action in production landscapes: priority areas in western New South Wales defined by irreplaceability and vulnerability to vegetation loss. Biological Conservation, 100(01), 345-376.

Pressey, R. L., Cabeza, M., Watts, M. E., Cowling, R. M., \& Wilson, K. a. (2007). Conservation planning in a changing world. Trends in Ecology \& Evolution, 22(11), 583-92. http://doi.org/10.1016/j.tree.2007.10.001

R Core Team (2012). R: A language and environment for statistical computing. R Foundation for Statistical Computing, Vienna, Austria. ISBN 3-900051-07-0, http://www.R-project.org/

Rangel, T., Bini, L., Dinizfilho, J., Pinto, M., Carvalho, P., \& Bastos, R. (2007). Human development and biodiversity conservation in Brazilian Cerrado. Applied Geography, 27(1), 14-27. http://doi.org/10.1016/j.apgeog.2006.09.009 
Rêdo, D., Aide, T. M., \& Clark, M. L. (2013). Vegetation change in Brazil's dryland ecoregions and the relationship to crop production and environmental factors : Cerrado, Caating, and Mato Grosso, 2001 - 2009. Journal of Land Use Science, (June 2013), 37-41.

Radosavljevic, A., \& Anderson, R. P. (2014). Making better Maxent models of species distributions: Complexity, overfitting and evaluation. Journal of Biogeography, 41(4), 629-643. http://doi.org/10.1111/jbi.12227

Rondinini, C., Wilson, K. a, Boitani, L., Grantham, H., \& Possingham, H. P. (2006). Tradeoffs of different types of species occurrence data for use in systematic conservation planning. Ecology Letters, 9(10), 1136-45. http://doi.org/10.1111/j.1461-0248.2006.00970.x

Sano, E. E., Rosa, R., Brito, J. L. S., \& Ferreira, L. G. (2010). Land cover mapping of the tropical savanna region in Brazil. Environmental Monitoring and Assessment, 166(1-4), 113-24. http://doi.org/10.1007/s10661-009-0988-4

Sarkar, S., \& Illoldi-Rangel, P. (2010). Systematic Conservation Planning: an Updated Protocol. Natureza \& Conservação, 08(01), 19-26. http://doi.org/10.4322/natcon.00801003

SpeciesLink (2015) http://www.splink.org.br/index?lang=pt. Acessado em 02/09/2015.

Urbina-Cardona, J.N., Flores_Villela, O. (2010). Ecological-niche modeling and priorization of conservation-area networks for Mexican herpetofauna. Conservation Biology, 24, 1031-1041.

Silva, J.M. C. da; Bates, J. M. (2002). Biogeographic Patterns and Conservation in the South American Cerrado: A Tropical Savanna Hotspot. BioScience, 52(3), 225. http://doi.org/10.1641/00063568(2002)052[0225:BPACIT]2.0.CO;2

Silva, R.D.J., Cardoso, L., Vieira, G., Pinto, M.P., 2006. Malha rodoviária e conflitos de conservação no cerrado: um estudo para a preservação de anfíbios. Acta Sci.Biol.Sci. 373-378.

Valdujo, P. H., Silvano, D. L., Colli, G., \& Martins, M. (2012). Anuran Species Composition and Distribution Patterns in Brazilian Cerrado, a Neotropical Hotspot. South American Journal of Herpetology, 7(2), 63-78. http://doi.org/10.2994/057.007.0209

Verburg, P. H., Overmars, K. P., Huigen, M. G. a., de Groot, W. T., \& Veldkamp, a. (2006). Analysis of the effects of land use change on protected areas in the Philippines. Applied Geography, 26(2), 153173. http://doi.org/10.1016/j.apgeog.2005.11.005 


\section{MATERIAL SUPLEMENTAR}

Tabela S1: Metas de Aichi (COP 10) - 2011-2020 e as metas brasileiras (Resolução CONABIO no.6, de 03/07/2013) (adaptado de Han et al., 2014); * Estrutura de classificação OECD (Linster 2003)

\begin{tabular}{|c|c|c|c|}
\hline INDICADORES* & PRESSÃO & ESTADO & RESPOSTA \\
\hline \multirow[t]{2}{*}{$\begin{array}{l}\text { METAS DE } \\
\text { AICHI }\end{array}$} & \multirow[t]{2}{*}{$\begin{array}{c}\text { Meta 5: "Em 2020, a taxa de } \\
\text { perda de todos os hábitats } \\
\text { naturais, incluindo as florestas, } \\
\text { deverá ser, pelo menos a } \\
\text { metade e sempre que possível, } \\
\text { levada para perto de zero, e a } \\
\text { degradação e a fragmentação } \\
\text { serão significativamente } \\
\text { reduzidas" }\end{array}$} & \multirow[t]{2}{*}{$\begin{array}{l}\text { Meta 12: "Em 2020, a extinção de espécies } \\
\text { ameaçadas conhecidas terá sido evitada e } \\
\text { seu estado de conservação, em especial, } \\
\text { aquelas mais em declínio, será melhorada }\end{array}$} & $\begin{array}{l}\text { Meta 11: "Em 2020, pelo menos } 17 \% \\
\text { das áreas terrestres, áreas de particular } \\
\text { importância para a biodiversidade e } \\
\text { serviços ecossistêmicos, conservadas por } \\
\text { um sistema de áreas protegidas (e outras } \\
\text { áreas importantes para a conservação), } \\
\text { ecologicamente representativos, } \\
\text { satisfatoriamente interligados". }\end{array}$ \\
\hline & & & $\begin{array}{l}\text { Meta 15: "restaurar, pelo menos, } 15 \% \\
\text { das áreas degradadas" }\end{array}$ \\
\hline \multirow{3}{*}{$\begin{array}{c}\text { METAS } \\
\text { BRASILEIRAS }\end{array}$} & \multirow{3}{*}{$\begin{array}{l}\text { "Até } 2002 \text { a taxa de perda de } \\
\text { ambientes nativos será } \\
\text { reduzida em pelo menos } 50 \% \\
\text { (em relação à 2009) e, na } \\
\text { medida do possível, levada a } \\
\text { perto de zero e a degradação e } \\
\text { fragmentação terão sido } \\
\text { reduzidas significativamente } \\
\text { em todos os biomas" }\end{array}$} & \multirow{3}{*}{$\begin{array}{c}\text { "O risco de extinção de espécies } \\
\text { ameaçadas terá sido reduzido } \\
\text { significativamente, tendendo a zero, e sua } \\
\text { situação de conservação, em especial } \\
\text { daquelas sofrendo maior declínio, terá sido } \\
\text { melhorada" }\end{array}$} & "Pelo menos $17 \%$ do bioma Cerrado \\
\hline & & & $\begin{array}{c}\text { protegido por Unidades de Conservação } \\
\text { do SNUC, APPs, Reservas Legais e } \\
\text { Terras Indígenas, principalmente em } \\
\text { áreas de especial importância para a } \\
\text { biodiversidade e serviços } \\
\text { ecossistêmicos, visando garantir a } \\
\text { interligação, integração e representação } \\
\text { ecológica em paisagens terrestres”. }\end{array}$ \\
\hline & & & $\begin{array}{c}\text { "restaurar, pelo menos, } 15 \% \text { das áreas } \\
\text { degradadas" }\end{array}$ \\
\hline $\begin{array}{c}\text { MODO DE } \\
\text { AVALIAÇÃOO }\end{array}$ & $\begin{array}{c}\text { Área remanescente nos } \\
\text { cenários LUCC (BAU e GOV.) } \\
2020 \text { e } 2050 .\end{array}$ & $\begin{array}{c}\text { RLI (Brasil, Cerrado e Endêmicas de } \\
\text { Cerrado) e Perda de área futura das } \\
\text { espécies. }\end{array}$ & $\begin{array}{c}\text { Proporção de áreas reservadas para a } \\
\text { proteção de } 17 \% \text { dos SDMs das } \\
\text { espécies-alvo. }\end{array}$ \\
\hline
\end{tabular}


Tabela S2: Lista de espécies-alvo selecionadas no estudo, de acordo com critérios de risco de extinção da IUCN (Lista Nacional - L.N.) e endemismo no Cerrado.

\begin{tabular}{|c|c|c|c|c|}
\hline \multirow[b]{2}{*}{$\frac{\pi}{2}$} & ESPÉCIE & L.N. & CRITÉRIO IUCN & ENDÊEMICA \\
\hline & $\begin{array}{l}\text { Alectrurus tricolor (Vieillot, 1816) } \\
\text { Alipiopsitta xanthops (Spix, 1824) } \\
\text { Anodorhynchus hyacinthinus (Latham, 1790) } \\
\text { Asthenes luizae (Vielliard, 1990) } \\
\text { Celeus obrieni (Short, 1973) } \\
\text { Cercomacra ferdinandi (Snethlage, 1928) } \\
\text { Columbina cyanopis (Pelzeln, 1870) } \\
\text { Conothraupis mesoleuca (Berlioz, 1939) } \\
\text { Euscarthmus rufomarginatus (Pelzeln, 1868) } \\
\text { Geositta poeciloptera (Wied, 1830) } \\
\text { Hydropsalis candicans (Pelzeln, 1867) } \\
\text { Nothura minor } \text { (Spix, 1825) } \\
\text { Penelope ochrogaster (Pelzeln, 1870) } \\
\text { Phylloscartes roquettei (Snethlage, 1928) } \\
\text { Pyrrhura pfrimeri (Miranda-Ribeiro, 1920) } \\
\text { Scytalopus novacapitalis } \text { (Sick, 1958) } \\
\text { Taoniscus nanus } \text { (Temminck, 1815) }\end{array}$ & $\begin{array}{l}\text { VU } \\
\text { NT } \\
\text { NT } \\
\text { NT } \\
\text { VU } \\
\text { VU } \\
\text { CR } \\
\text { EN } \\
\text { NT } \\
\text { EN } \\
\text { VU } \\
\text { EN } \\
\text { VU } \\
\text { EN } \\
\text { EN } \\
\text { EN } \\
\text { EN }\end{array}$ & $\begin{array}{l}\text { B2ab(ii,iii) } \\
- \\
- \\
- \\
\text { C1 } \\
\text { A3c } \\
\text { D } \\
\text { A3c } \\
- \\
\text { B2ab(ii,iii) } \\
\text { D2 } \\
\text { B2ab(ii,iii) } \\
\text { C1+2a(i) } \\
\text { B2ab(iii) } \\
\text { A4c } \\
\text { B2ab(iii) } \\
\text { B2ab(ii,iii) }\end{array}$ & Lopes, 2009 \\
\hline 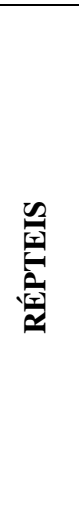 & $\begin{array}{l}\text { Apostolepis serrana (De Lema \& Renner, 2006) } \\
\text { Apostolepis striata (De Lema, 2004) } \\
\text { Ameiva parecis (Colli, Costa, Garda, Kopp, Mesquita, Peres, Valdujo, Vieira \& Wiederhecker, 2003) } \\
\text { Bachia didactyla (Freitas Strüssmann, Carvalho, Kawashita-Ribeiro \& Mott, 2011) } \\
\text { Bachia psamophila (Rodrigues, Pavan \& Curcio, 2007) } \\
\text { Bothrops itapetiningae (Boulenger, 1907) } \\
\text { Heterodactylus lundii (Reinhardt \& Lütken, 1862) } \\
\text { Hydrodynastes melanogigas (Franco, Fernandes \& Bentim, 2007) } \\
\text { Kentropyx vanzoi (Gallagher \& Dixon, 1980) } \\
\text { Phalotris lativittatus (Ferrarezzi, 1993) } \\
\text { Phalotris multipunctatus (Puorto \& Ferrarezzi, 1993) } \\
\text { Philodryas livida (Amaral, 1923) } \\
\text { Placosoma cipoense (Cunha, 1966) } \\
\text { Liotyphlops schubarti (Vanzolini, 1948) }\end{array}$ & $\begin{array}{l}\text { EN } \\
\text { EN } \\
\text { EN } \\
\text { EN } \\
\text { CR } \\
\text { NT } \\
\text { VU } \\
\text { EN } \\
\text { VU } \\
\text { NT } \\
\text { EN } \\
\text { VU } \\
\text { EN } \\
\text { NT }\end{array}$ & $\begin{array}{l}\text { B1ab(iii) } \\
\text { B1ab(iii) } \\
\text { B1ab(iii) } \\
\text { B1ab(iii) } \\
\text { A2c } \\
- \\
\text { B1ab(iii) } \\
\text { B1ab(iii) } \\
\text { A2c } \\
- \\
\text { B1ab(iii) } \\
\text { A2c } \\
\text { B1ab(iii) }\end{array}$ & Nogueira et al., 2010 \\
\hline 偪 & $\begin{array}{l}\text { Allobates goianus (Bokermann, 1975) } \\
\text { Phyllomedusa centralis (Bokermann, 1965) } \\
\text { Proceratophrys moratoi (Jim \& Caramaschi, 1980) } \\
\text { Bokermannohyla martinsi (Bokermann, 1964) }\end{array}$ & $\begin{array}{l}\text { EN } \\
\text { NT } \\
\text { EN } \\
\text { NT }\end{array}$ & $\begin{array}{l}\text { B1ab(iii,iv) } \\
\text { B1b(iii) } \\
\text { B1ab(i,iii) } \\
\text { - }\end{array}$ & Valdujo, 2011 \\
\hline \multirow{2}{*}{ 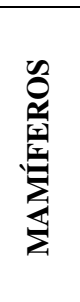 } & \multirow{2}{*}{$\begin{array}{l}\text { Glyphonycteris behnii (Peters, 1865) } \\
\text { Gyldenstolpia planaltensis (Âvila-Pires, 1972) } \\
\text { Phyllomys brasiliensis (Lund, 1840) } \\
\text { Euryoryzomys lamia (Thomas, 1901) } \\
\text { Juscelinomys candango (Moojen, 1965) } \\
\text { Kerodon acrobata (Moojen, Locks \& Langguth, 1997) } \\
\text { Lonchophylla bokermanni (Sazima, Vizotto \& Taddei, 1978) } \\
\text { Lycalopex vetulus (Lund, 1842) }\end{array}$} & $\begin{array}{l}\text { VU } \\
\text { EN } \\
\text { EN } \\
\text { EN }\end{array}$ & \multirow{2}{*}{$\begin{array}{l}\text { A4c } \\
\text { B2ab(iii,iv) } \\
\text { B2ab(iii,iv) } \\
\text { B1ab(iii) } \\
\text { D } \\
\text { B1ab(iii,v) } \\
- \\
\text { A2cd+3cd }\end{array}$} & Bonvicino et al., 2012 \\
\hline & & $\begin{array}{l}\text { CR } \\
\text { VU } \\
\text { NT } \\
\text { VU }\end{array}$ & & Paglia et al., 2012 \\
\hline
\end{tabular}




\begin{tabular}{|l|l|l|l|l|}
\hline \multicolumn{1}{|c|}{ ESPÉCIE } & L.N. & \multicolumn{1}{|c|}{ CRITÉRIO IUCN } \\
\hline \multirow{3}{*}{} & Microakodontomys transitorius (Hershkovitz, 1993) & EN & B1ab(iii)+2ab(iii) \\
& Oligoryzomys rupestris (Weksler \& Bonvicino, 2005) & EN & B2ac(iii,iv) \\
& Thalpomys cerradensis (Hershkovitz, 1990) & VU & A2c+A3c \\
& Thalpomys lasiotis (Thomas, 1916) & EN & A2bc \\
& Thylamys velutinus (Wagner, 1842) & VU & A2c \\
\hline
\end{tabular}

Tabela S3: Descrição das variáveis ambientais utilizadas nos SDMs por táxon - (BIO 2, BIO 3, BIO 6, BIO 7 não foram selecionadas para gerar os modelos).

\begin{tabular}{|l|l|l|l|}
\hline & VARIÁVEL & \multicolumn{1}{|c|}{ DESCRIÇÃO } & \multicolumn{1}{c|}{ TÁXON } \\
\hline & BIO 1 & Temperatura média anual & Mamíferos \\
& BIO 2 & Variação media diurna da temperatura & - \\
& BIO 3 & Isotermalidade (BIO 2/BIO 7) *100) & Aves, Répteis, Mamíferos \\
& BIO 4 & Sazonalidade de temperatura (desvio padrão*100) & Aves, Répteis, Anfíbios \\
& BIO 5 & Temperatura máxima do mês mais quente & - \\
& BIO 6 & Temperatura mínima do mês mais frio & - \\
& BIO 7 & Variação anual da temperatura (BIO 5 - BIO 6) & Mamíferos \\
& BIO 10 & Temperatura média do trimestre mais quente & Aves, Répteis, Anfíbios \\
& BIO 11 & Temperatura média do trimestre mais frio & Anfíbios \\
& BIO 12 & Precipitação anual & Anfíbios \\
& BIO 13 & Precipitação do mês mais chuvoso & Aves, Répteis, Mamíferos, Anfíbios \\
& BIO 14 & Precipitação do mês mais seco & Mamíferos, Anfíbios \\
& BIO 15 & Sazonalidade da precipitação (coeficiente de variação) & Mamíferos \\
& BIO 16 & Precipitação do trimestre mais úmido & Aves, Répteis, Mamíferos, Anfíbios \\
\hline
\end{tabular}


Tabela S4: RLI de todas as espécies, que ocorrem no Cerrado e endêmicas desse bioma, entre 2003 e 2014 (n=número de espécies avaliadas).

\begin{tabular}{|cc|cc|ccc|}
\hline & \multicolumn{2}{c}{ BRASIL } & \multicolumn{2}{c}{ CERRADO } & \multicolumn{2}{c|}{ ENDÊMICAS } \\
\hline \multirow{2}{*}{ TODAS } & 2003 & 2014 & 2003 & 2014 & 2003 & 2014 \\
& 0,7209 & 0,5595 & - & - & 0.7105 & 0.5474 \\
& $\mathrm{n}=526$ & $\mathrm{n}=126$ & \multicolumn{2}{c}{$\mathrm{n}=38$} \\
\hline \multirow{2}{*}{ AVES } & 0.6959 & 0.5849 & 0.7159 & 0.6464 & 0.6 & 0.5445 \\
& $\mathrm{n}=292$ & $\mathrm{n}=69$ & $\mathrm{n}=18$ \\
\hline MAMÍFEROS & 0.704 & 0.5134 & 0.7905 & 0.5952 & 0.7778 & 0.5334 \\
& $\mathrm{n}=127$ & $\mathrm{n}=42$ & $\mathrm{n}=9$ \\
\hline \multirow{2}{*}{ ANFÍBIOS } & 0.7756 & 0.561 & 0.8857 & 0.5714 & 0.8 & 0.6 \\
& $\mathrm{n}=41$ & $\mathrm{n}=7$ & $\mathrm{n}=4$ \\
\hline \multirow{2}{*}{ RÉPTEIS } & 0.8212 & 0.5334 & 0.8889 & 0.6 & 0.8571 & 0.5428 \\
& $\mathrm{n}=66$ & $\mathrm{n}=8$ & $\mathrm{n}=7$ \\
\hline
\end{tabular}

Tabela S5: Comparação das listas de espécies e subespécies ameaçadas brasileira, anos de 2003 e 2014, sua ocorrência no Cerrado e endemismo neste bioma. Símbolos: Categorias IUCN (LC - Menos preocupante; NT - Próxima da ameaça; VU - Vulnerável; EN - Em perigo; CR - Criticamente em perigo; EW e EX - Extinta na natureza e Extinta; $\mathrm{RC}^{*}$ - Recém descrita; NA** - Não avaliada; E*** - Endemismo.

\begin{tabular}{|c|c|c|c|c|}
\hline & ESPÉCIE & 2003 & 2014 & CERRADO \\
\hline & Akodon mystax (Hershkovitz, 1998) & LC & VU & \\
\hline & Alouatta belzebul (Linnaeus, 1766) & $\mathrm{CR}$ & VU & \\
\hline & Alouatta caraya (Humboldt, 1812) & LC & NT & $\checkmark$ \\
\hline & Alouatta discolor (Spix, 1823) & LC & VU & \\
\hline & Alouatta guariba clamitans (Cabrera, 1940) & NT & VU & \\
\hline & Alouatta guariba guariba (Humboldt, 1812) & $\mathrm{CR}$ & CR & \\
\hline & Alouatta puruensis (Lönnberg, 1941) & LC & NT & \\
\hline & Alouatta ululata (Elliot, 1912) & $\mathrm{CR}$ & EN & \\
\hline & Ateles belzebuth (É. Geoffroy, 1806) & VU & VU & \\
\hline & Ateles chamek (Humboldt, 1812) & LC & VU & \\
\hline & Ateles marginatus (É. Geoffroy, 1809) & EN & EN & \\
\hline & Atelocynus microtis (Sclater, 1883) & DD & VU & $\mathrm{NA}^{* *}$ \\
\hline & Balaenoptera borealis (Lesson, 1828) & VU & EN & \\
\hline & Balaenoptera musculus (Linnaeus, 1758) & $\mathrm{CR}$ & $\mathrm{CR}$ & \\
\hline & Balaenoptera physalus (Linnaeus, 1758) & EN & EN & \\
\hline & Blastocerus dichotomus (Illiger, 1815) & VU & $\mathrm{VU}$ & $\checkmark$ \\
\hline & Brachyteles arachnoides (É. Geoffroy, 1806) & EN & EN & \\
\hline & Brachyteles hypoxanthus (Khul, 1820) & $\mathrm{CR}$ & $\mathrm{CR}$ & \\
\hline & Bradypus torquatus (Illiger, 1811) & VU & $\mathrm{VU}$ & \\
\hline & Cacajao calvus calvus (I. Geoffroy, 1847) & VU & $\mathrm{LC}$ & \\
\hline b & Cacajao calvus novaesi (Hershkovitz, 1987) & VU & DD & NA \\
\hline$\frac{1}{2}$ & Cacajao calvus rubicundus (I. Geoffroy \& Deville, 1848) & VU & $\mathrm{LC}$ & \\
\hline 兴 & Callicebus barbarabrownae (Hershkovitz, 1990) & $\mathrm{CR}$ & $\mathrm{CR}$ & \\
\hline$\$$ & Callicebus brunneus (Wagner, 1842) & LC & NT & \\
\hline$\Sigma$ & Callicebus coimbrai (Kobayashi \& Langguth, 1999) & $\mathrm{CR}$ & EN & \\
\hline
\end{tabular}


Callicebus melanochir (Wied-Neuwied, 1820)

Callicebus nigrifrons (Spix, 1823)

Callicebus personatus (É. Geoffroy, 1812)

Callimico goeldii (Thomas, 1904)

Callistomys pictus (Pictet, 1841)

Callithrix aurita (É. Geoffroy in Humboldt, 1812)

Callithrix flaviceps (Thomas, 1903)

Callithrix kuhlii (Coimbra-Filho, 1985)

Caluromysiops irrupta (Sanborn, 1951)

Carterodon sulcidens (Lund, 1841)

Cavia intermedia (Cherem, Olimpio \& Ximenez, 1999)

Cavia magna (Ximenez, 1980)

Cebus kaapori (Queiroz, 1992)

Chaetomys subspinosus (Olfers, 1818)

Chiropotes albinasus (I. Geoffroy \& Deville, 1848)

Chiropotes satanas (Hoffmannsegg, 1807)

Chiropotes utahicki (Hershkovitz, 1985)

Chrysocyon brachyurus (Illiger, 1815)

Ctenomys bicolor (Waterhouse, 1848)

Ctenomys flamarioni (Travi, 1981)

Ctenomys ibicuiensis (Freitas, Fernandes, Fornel \& Roratto, 2012)

Ctenomys lami (Freitas, 2001)

Ctenomys minutus (Nehring, 1887)

Dasyprocta azarae (Lichtenstein, 1823)

Eubalaena australis (Desmoulins, 1822)

Euryoryzomys lamia (Thomas, 1901)

Furipterus horrens (F. Cuvier, 1828)

Glyphonycteris behnii (Peters, 1865)

Hyleaamys oniscus (Thomas, 1904)

Inia geoffrensis (de Blainville, 1817)

Juliomys rimofrons (Oliveira \& Bonvicino, 2002)

Juscelinomys candango (Moojen, 1965)

Kerodon acrobata (Moojen, Locks \& Langguth, 1997)

Kerodon rupestris (Wied-Neuwied, 1820)

Kunsia fronto (Winge, 1887)/ Gyldenstolpia planaltensis

Lagothrix cana cana (É. Geoffroy in Humboldt, 1812)

Lagothrix lagothricha (Humboldt, 1812)

Lagothrix poeppigii (Schinz, 1844)

Lasiurus ebenus (Fazzolari-Corrêa, 1994)

Leontopithecus caissara (Lorini \& Persson, 1990)

Leontopithecus chrysomelas (Kuhl, 1820)

Leontopithecus chrysopygus (Mikan, 1823)

Leontopithecus rosalia (Linnaeus, 1766)

Leopardus (Oncifelis) geoffroyi (d'Orbigny \& Gervais, 1844)

Leopardus colocolo (Molina, 1782)

Leopardus guttulus (Hensel, 1872)

Leopardus tigrinus (Schreber, 1775)

Leopardus wiedii (Schinz, 1821)

Lonchophylla bokermanni (Sazima, Vizotto \& Taddei, 1978)

\begin{tabular}{|c|c|c|}
\hline $\mathrm{VU}$ & VU & \\
\hline NT & $\mathrm{LC}$ & \\
\hline $\mathrm{VU}$ & VU & \\
\hline NT & $\mathrm{LC}$ & \\
\hline $\mathrm{VU}$ & EN & \\
\hline $\mathrm{VU}$ & EN & \\
\hline EN & EN & \\
\hline LC & NT & \\
\hline $\mathrm{CR}$ & CR & \\
\hline CR & DD & NA \\
\hline LC & $\mathrm{CR}$ & \\
\hline LC & NT & \\
\hline $\mathrm{CR}$ & $\mathrm{CR}$ & \\
\hline $\mathrm{VU}$ & VU & \\
\hline LC & NT & \\
\hline EN & CR & \\
\hline VU & VU & \\
\hline VU & VU & $\checkmark$ \\
\hline LC & EN & \\
\hline VU & EN & \\
\hline $\mathrm{RD}^{*}$ & NT & NA \\
\hline LC & EN & \\
\hline LC & VU & $\checkmark$ \\
\hline NT & $\mathrm{LC}$ & $\checkmark$ \\
\hline EN & EN & \\
\hline $\mathrm{DD}$ & EN & NA \\
\hline LC & VU & $\checkmark$ \\
\hline LC & VU & $\checkmark \mathrm{E}^{* * *}$ \\
\hline LC & NT & \\
\hline NT & EN & \\
\hline LC & NT & \\
\hline $\mathrm{CR}$ & $\mathrm{CR}$ & $\checkmark \mathrm{E}$ \\
\hline LC & VU & $\checkmark \mathrm{E}$ \\
\hline LC & VU & \\
\hline $\mathrm{CR}$ & EN & $\checkmark \mathrm{E}$ \\
\hline NT & EN & \\
\hline NT & VU & \\
\hline NT & VU & \\
\hline VU & DD & NA \\
\hline CR & EN & \\
\hline EN & EN & \\
\hline CR & EN & \\
\hline EN & EN & \\
\hline NT & VU & \\
\hline VU & VU & $\checkmark$ \\
\hline LC & VU & $\checkmark$ \\
\hline VU & EN & $\checkmark$ \\
\hline $\mathrm{VU}$ & VU & $\checkmark$ \\
\hline VU & NT & $\checkmark \mathrm{E}$ \\
\hline
\end{tabular}


Lonchophylla dekeyseri (Taddei, Vizotto \& Sazima, 1983)

Lonchorhina aurita (Tomes, 1863)

Lontra longicaudis (Olfers, 1818)

Lycalopex vetulus (Lund, 1842)

Marmosops ocellatus (Tate, 1931)

Marmosops paulensis (Tate, 1931)

Mazama bororo (Duarte, 1996)

Mazama nana (Hensel, 1872)

Megaptera novaeangliae (Borowski, 1781)

Mico melanurus (É. Geoffroy em Humboldt, 1812)

Microakodontomys transitorius (Hershkovitz, 1993)

Monodelphis iheringi (Thomas, 1888)

Myotis ruber (Geoffroy, 1806)

Myrmecophaga tridactyla (Linnaeus, 1758)

Natalus macrourus (espiritosantensis) (Gervais, 1856)

Oxymycterus caparaoe (Hershkovitz, 1998)

Ozotoceros bezoarticus bezoarticus (Linnaeus, 1758)

Ozotoceros bezoarticus leucogaster (Goldfuss, 1817)

Panthera onca (Linnaeus, 1758)

Phaenomys ferrugineus (Thomas, 1894)

Phyllomys brasiliensis (Lund, 1840)

Phyllomys thomasi (Ihering, 1871)

Phyllomys unicolor (Wagner, 1842)

Physeter macrocephalus Linnaeus, 1758

Platyrrhinus recifinus (Thomas, 1901)

Pontoporia blainvillei (Gervais \& d'Orbigny, 1844)

Priodontes maximus (Kerr, 1792)

Pteronura brasiliensis (Gmelin, 1788)

Puma (Herpailurus/ Felis) yagouarondi (É. Geoffroy, 1803)

Puma concolor (Linnaeus, 1771)

Reithrodon typicus (Waterhouse, 1837)

Rhagomys rufescens (Thomas, 1886)

Saguinus bicolor (Spix, 1823)

Saguinus martinsi martinsi (Thomas, 1912)

Saguinus martinsi ochraceus (Hershkovitz, 1966)

Saguinus niger (É. Geoffroy, 1803)

Saimiri ustus (I. Geoffroy, 1843)

Saimiri vanzolinii (Ayres, 1985)

Sapajus (Cebus) robustus (Kuhl, 1820)

Sapajus (Cebus) xanthosternos (Wied-Neuwied, 1826)

Sapajus cay (Illiger, 1815)

Sapajus flavius (Schreber, 1774)

Sapajus libidinosus (Spix, 1823)

Sapajus nigritus cucullatus (Spix, 1823)

Sapajus nigritus nigritus (Goldfuss, 1809)

Sotalia fluviatilis (Gervais \& Deville, 1853)

Sotalia guianensis (Van Beneden, 1864)

Speothos venaticus (Lund, 1842)

Tapirus terrestris (Linnaeus, 1758)

\begin{tabular}{|l|l|l|} 
VU & EN & $\checkmark$ \\
LC & VU & $\checkmark$ \\
NT & NT & $\checkmark$ \\
LC & VU & $\checkmark$ E \\
LC & NT & $\checkmark$ \\
LC & VU & \\
LC & VU & \\
VU & VU & \\
LC & NT & \\
LC & NT & $\checkmark$ \\
LC & EN & $\checkmark$ E \\
LC & NT & \\
VU & LC & \\
VU & VU & $\checkmark$ \\
LC & VU & \\
LC & NT & \\
NT & VU & $\checkmark$ \\
NT & VU & \\
VU & VU & $\checkmark$ \\
VU & DD & NA \\
EN & EN & $\checkmark \mathrm{E}$ \\
EN & EN & \\
CR & CR & \\
VU & VU & \\
VU & LC & $\checkmark$ \\
EN & CR & \\
VU & VU & $\checkmark$ \\
VU & VU & $\checkmark$ \\
LC & VU & $\checkmark$ \\
VU & VU & $\checkmark$ \\
LC & NT & \\
VU & LC & \\
CR & CR & \\
LC & NT & \\
LC & NT & \\
LC & VU & $\checkmark$ \\
LC & NT & \\
VU & VU & \\
VU & EN & \\
CR & EN & \\
LC & VU & \\
LC & EN & \\
LC & NT & $\checkmark$ \\
NT & NT & \\
NT & NT & \\
NT & NT & \\
VU & VU & \\
& & \\
& \\
LU & $\checkmark$ \\
&
\end{tabular}




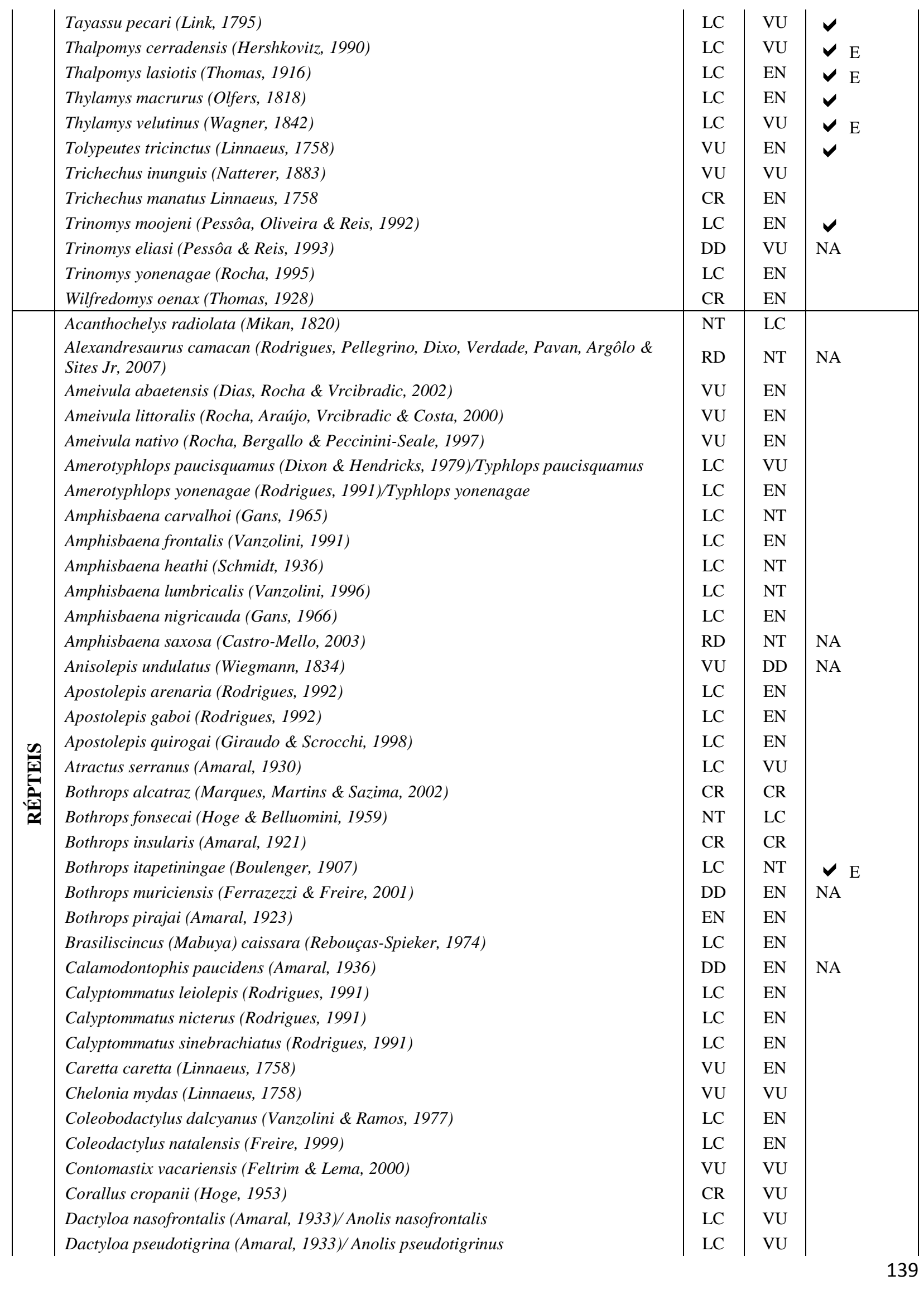




\begin{tabular}{|c|c|c|c|c|}
\hline & Dermochelys coriacea (Linnaeus, 1766) & $\mathrm{CR}$ & $\mathrm{CR}$ & \\
\hline & Dipsas albifrons (cavalheiroi) Hoge, 1950 & $\mathrm{CR}$ & $\mathrm{LC}$ & \\
\hline & Ditaxodon taeniatus (Peters, 1868) & $\mathrm{DD}$ & VU & NA \\
\hline & Echinanthera cephalomaculata (Di-Bernardo, 1994) & DD & VU & NA \\
\hline & Eretmochelys imbricata (Linnaeus, 1766) & $\mathrm{EN}$ & $\mathrm{CR}$ & \\
\hline & Eurolophosaurus amathites (Rodrigues, 1984) & $\mathrm{LC}$ & $\mathrm{EN}$ & \\
\hline & Gonatodes tapajonicus (Rodrigues, 1980) & $\mathrm{LC}$ & EN & \\
\hline & Gymnophthalmus leucomystax (Vanzolini \& Carvalho, 1991) & $\mathrm{LC}$ & NT & \\
\hline & Heterodactylus lundii (Reinhardt \& Lütken, 1862) & VU & VU & $\checkmark \mathrm{E}$ \\
\hline & Homonota uruguayensis (Vaz-Ferreira \& Sierra de Soriano, 1961) & $\mathrm{LC}$ & VU & \\
\hline & Hydromedusa maximiliani (Mikan, 1820) & NT & $\mathrm{LC}$ & \\
\hline & Kentropyx vanzoi (Gallagher \& Dixon, 1980) & $\mathrm{LC}$ & VU & $\checkmark \mathrm{E}$ \\
\hline & Lepidochelys olivacea (Eschscholtz, 1829) & $\mathrm{EN}$ & $\mathrm{EN}$ & \\
\hline & Leposoma annectans (Ruibal, 1952) & $\mathrm{LC}$ & VU & \\
\hline & Leposoma baturitensis (Rodrigues \& Borges, 1997) & DD & $\mathrm{EN}$ & NA \\
\hline & Leposoma nanodactylus (Rodrigues, 1997) & $\mathrm{LC}$ & $\mathrm{EN}$ & \\
\hline & Leposternon (Amphisbaena) kisteumacheri (Porto, Soares \& Caramaschi, 2000) & $\mathrm{LC}$ & VU & \\
\hline & Leposternon octostegum (Duméril, 1851)/ Amphisbaena octostegum & $\mathrm{LC}$ & $\mathrm{EN}$ & \\
\hline & Leposternon scutigerum (Hemprich, 1820)/ Amphisbaena scutigerum & $\mathrm{LC}$ & $\mathrm{EN}$ & \\
\hline & Liolaemus lutzae (Mertens, 1938) & $\mathrm{CR}$ & $\mathrm{CR}$ & \\
\hline & Liolaemus occipitalis (Boulenger, 1885) & VU & VU & \\
\hline & Mesoclemmys hogei (Mertens, 1967) & $\mathrm{EN}$ & $\mathrm{CR}$ & \\
\hline & Mussurana (Clelia) montana (Franco, Marques \& Puorto, 1997) & NT & $\mathrm{LC}$ & \\
\hline & Phalotris multipunctatus (Puorto \& Ferrarezzi, 1993) & $\mathrm{LC}$ & $\mathrm{EN}$ & $\checkmark \mathrm{E}$ \\
\hline & Philodryas livida (Amaral, 1923) & $\mathrm{LC}$ & VU & $\checkmark \mathrm{E}$ \\
\hline & Placosoma cipoense (Cunha, 1966) & $\mathrm{EN}$ & $\mathrm{EN}$ & $\checkmark \mathrm{E}$ \\
\hline & Podocnemis expansa (Schweigger, 1812) & $\mathrm{LC}$ & NT & $\checkmark$ \\
\hline & Podocnemis sextuberculata (Cornalia, 1849) & $\mathrm{LC}$ & NT & \\
\hline & Podocnemis unifilis (Troschel, 1848) & $\mathrm{LC}$ & NT & \\
\hline & Procellosaurinus tetradactylus (Rodrigues, 1991) & $\mathrm{LC}$ & $\mathrm{EN}$ & \\
\hline & Psilophthalmus paeminosus (Rodrigues, 1991) & $\mathrm{LC}$ & NT & \\
\hline & Rodriguesophis chui (Rodrigues, 1993) & $\mathrm{LC}$ & $\mathrm{EN}$ & \\
\hline & Rodriguesophis scriptorcibatus (Rodrigues, 1993) & $\mathrm{LC}$ & $\mathrm{EN}$ & \\
\hline & Stenocercus azureus (Müller, 1882) & $\mathrm{DD}$ & $\mathrm{EN}$ & NA \\
\hline & Stenocercus dumerilii (Steindachner, 1867) & $\mathrm{DD}$ & VU & NA \\
\hline & Trachemys adiutrix (Vanzolini, 1995) & $\mathrm{DD}$ & NT & NA \\
\hline & Trachemys dorbigni (Duméril \& Bibron, 1835) & $\mathrm{LC}$ & NT & \\
\hline & Tropidurus erythrocephalus (Rodrigues, 1987) & $\mathrm{LC}$ & VU & \\
\hline & Tropidurus hygomi (Reinhardt \& Luetken, 1861) & $\mathrm{LC}$ & VU & \\
\hline & Tropidurus jaguaribanus (Passos, Lima \& Borges-Nojosa, 2011) & $\mathrm{RD}$ & NT & NA \\
\hline & Tropidurus psammonastes (Rodrigues, Kasahara \& Yonenaga-Yasuda, 1988) & LC & EN & \\
\hline & Adelophryne baturitensis (Hoogmoed, Borges \& Cascon, 1994) & VU & LC & \\
\hline & Adelophryne maranguapensis (Hoogmoed, Borges, \& Cascon, 1994) & $\mathrm{EN}$ & VU & \\
\hline & Agalychnis granulosa (Cruz, 1989) & $\mathrm{CR}$ & VU & \\
\hline & Allobates brunneus (Cope, 1887) & $\mathrm{LC}$ & $\mathrm{CR}$ & $\checkmark$ \\
\hline$\underline{0}$ & Allobates goianus (Bokermann, 1975) & $\mathrm{LC}$ & $\mathrm{EN}$ & $\checkmark \mathrm{E}$ \\
\hline$\underline{\theta}$ & Allobates (Colostethus/ Eupemphix)olfersioides (A. Lutz, 1925)/ Phyllobates & DD & VU & NA \\
\hline Z & Aparasphenodon arapapa (Pimenta, Napoli \& Haddad, 2009) & $\mathrm{RD}$ & NT & NA \\
\hline 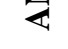 & Bokermannohyla (Hyla) martinsi (Bokermann, 1964) & $\mathrm{LC}$ & NT & $\checkmark \mathrm{E}$ \\
\hline
\end{tabular}


Bokermannohyla lucianae (Napoli \& Pimenta, 2003)

Bolitoglossa paraensis (altamazonica) (Unterstein, 1930)

Brachycephalus alipioi (Pombal \& Gasparini, 2006)

Brachycephalus pernix (Pombal, Wistuba \& Bornschein, 1998)

Ceratophrys ornata (Bell, 1843)

Chiasmocleis alagoanus (Cruz, Caramaschi \& Freire, 1999)

Crossodactylodes bokermanni (Peixoto, 1983)

Crossodactylus dantei (Carcerelli \& Caramaschi, 1993)

Crossodactylus lutzorum Carcerelli \& Caramaschi, 1993

Crossodactylus schmidti (Gallardo, 1961)

Cycloramphus bolitoglossus (Werner, 1897)

Cycloramphus diringshofeni (Bokermann, 1957)

Cycloramphus ohausi (Wandolleck, 1907)

Dendropsophus ruschii (Weygoldt \& Peixoto, 1987)

Euparkerella tridactyla (Izecksohn, 1988)

Holoaden bradei (B. Lutz, 1958)

Holoaden luederwaldti (Miranda-Ribeiro, 1920)

Hyla ( Bokermannohyla) izecksohni (Jim \& Caramaschi, 1979)

Hypsiboas cymbalum (Bokerman, 1963)

Hypsiboas poaju (Garcia, Peixoto \& Haddad, 2008)

Hypsiboas semiguttatus (A. Lutz, 1925)

Ischnocnema manezinho (Garcia, 1996)

Ischnocnema oea (Heyer, 1984)/Eleutherodactylus oeus

Megaelosia apuana (Pombal, Prado \& Canedo, 2003)

Megaelosia massarti (Witte, 1930)

Melanophryniscus cambaraensis (Braun \& Braun, 1979)

Melanophryniscus dorsalis (Mertens, 1933)

Melanophryniscus macrogranulosus (Braun, 1973)

Melanophryniscus montevidensis (Philippi, 1902)

Melanophryniscus spectabilis (Caramaschi \& Cruz, 2002)

Paratelmatobius lutzii (Lutz \& Carvalho, 1958)

Phasmahyla spectabilis (Cruz, Feio \& Nascimento, 2008)

Phyllodytes brevirostris (Peixoto \& Cruz, 1988)

Phyllodytes edelmoi (Peixoto, Caramaschi \& Freire, 2003)

Phyllomedusa ayeaye (B. Lutz, 1966)

Phyllomedusa centralis (Bokermann, 1965)

Physalaemus caete (Pombal \& Madureira, 1997)

Physalaemus maximus (Feio, Pombal, \& Caramaschi, 1999)

Physalaemus soaresi (Izecksohn, 1965)

Proceratophrys moratoi (Jim \& Caramaschi, 1980)

Proceratophrys palustris (Giaretta \& Sazima, 1993)

Scinax alcatraz (B. Lutz, 1973)

Scinax angrensis (B. Lutz, 1973)

Scinax duartei (B. Lutz, 1951)

Scinax ranki (Andrade \& Cardoso, 1987)

Thoropa lutzi (Cochran, 1938)

Thoropa petropolitana (Wandolleck, 1907)

Thoropa saxatilis (Crocoft \& Heyer, 1988)

Xenohyla truncata (Izecksohn, 1959)

\begin{tabular}{|c|c|c|}
\hline $\mathrm{RD}$ & NT & NA \\
\hline LC & EN & \\
\hline $\mathrm{RD}$ & NT & NA \\
\hline LC & $\mathrm{CR}$ & \\
\hline DD & NT & NA \\
\hline LC & EN & \\
\hline LC & NT & $\checkmark$ \\
\hline DD & EN & $\mathrm{NA}$ \\
\hline DD & $\mathrm{CR}$ & NA \\
\hline LC & NT & \\
\hline LC & NT & \\
\hline DD & $\mathrm{CR}$ & NA \\
\hline LC & EN & \\
\hline LC & NT & \\
\hline LC & NT & \\
\hline CR & $\mathrm{CR}$ & \\
\hline DD & EN & NA \\
\hline CR & $\mathrm{DD}$ & NA \\
\hline CR & CR & \\
\hline $\mathrm{RD}$ & NT & NA \\
\hline LC & EN & \\
\hline LC & VU & \\
\hline LC & NT & \\
\hline $\mathrm{RD}$ & NT & NA \\
\hline LC & NT & \\
\hline DD & VU & NA \\
\hline VU & $\mathrm{VU}$ & \\
\hline CR & EN & \\
\hline LC & NT & \\
\hline LC & NT & \\
\hline CR & $\mathrm{CR}$ & \\
\hline $\mathrm{RD}$ & NT & NA \\
\hline LC & NT & \\
\hline $\mathrm{RD}$ & NT & NA \\
\hline CR & LC & \\
\hline LC & NT & $\checkmark \mathrm{E}$ \\
\hline LC & EN & \\
\hline LC & $\mathrm{VU}$ & $\checkmark$ \\
\hline EN & $\mathrm{CR}$ & \\
\hline CR & EN & $\checkmark \mathrm{E}$ \\
\hline $\mathrm{LC}$ & CR & \\
\hline CR & $\mathrm{CR}$ & \\
\hline LC & NT & \\
\hline $\mathrm{LC}$ & VU & \\
\hline LC & NT & \\
\hline VU & DD & NA \\
\hline EN & EN & \\
\hline NT & VU & \\
\hline $\mathrm{LC}$ & EN & \\
\hline
\end{tabular}




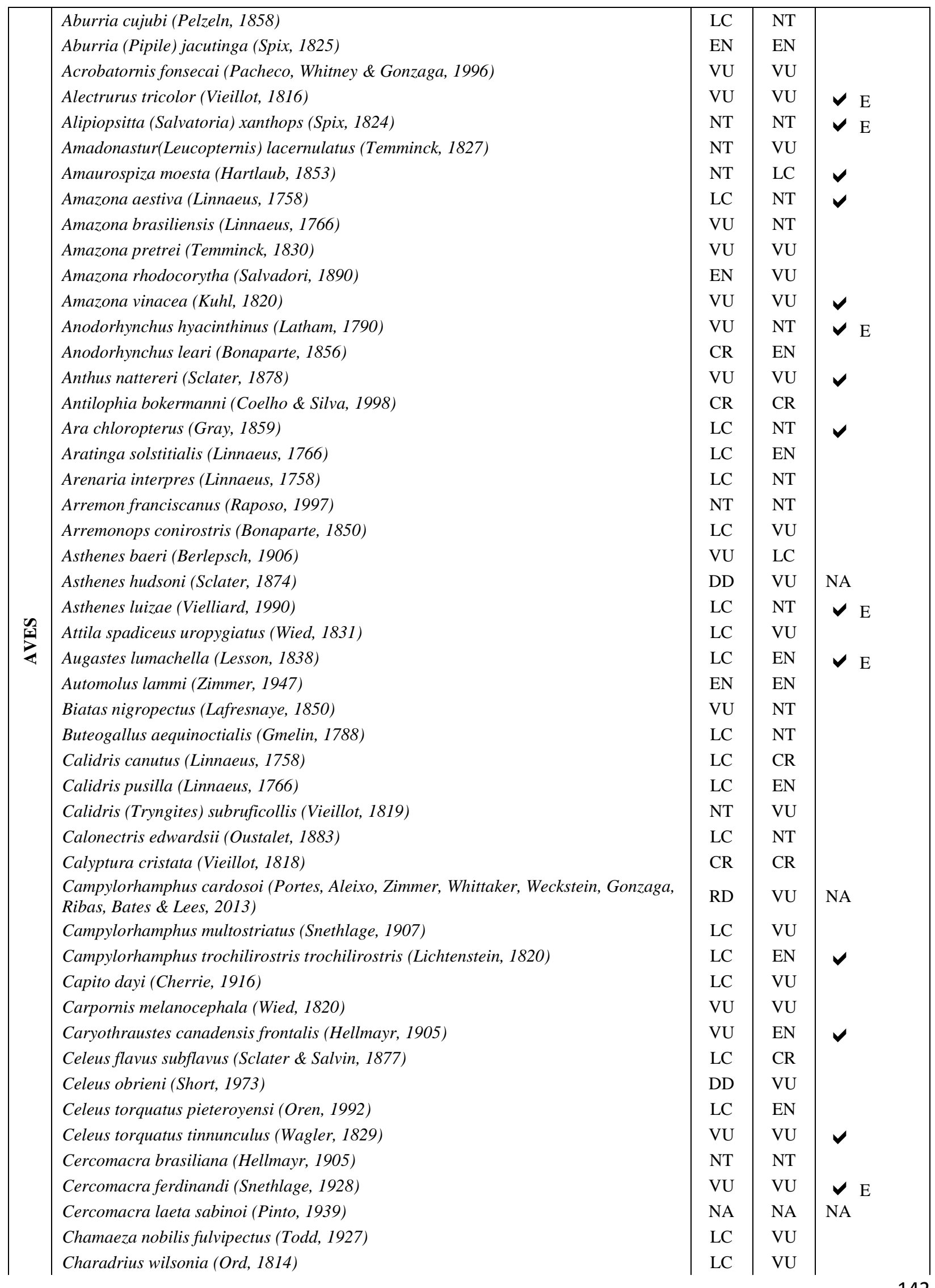


Cichlopsis leucogenys (Cabanis, 1851)

Cinclodes espinhacensis (Freitas, Chaves, Costa, Santos \& Rodrigues, 2012)

Cinclodes pabsti (Sick, 1969)

Circus cinereus (Vieillot, 1816)

Claravis geoffroyi (godefrida) (Temminck, 1811)

Clibanornis dendrocolaptoides (Pelzeln, 1859)

Columbina cyanopis (Pelzeln, 1870)

Conopophaga lineata cearae (Cory, 1916)

Conopophaga lineata lineata (Wied, 1831)

Conopophaga melanops nigrifrons (Pinto, 1954)

Conothraupis mesoleuca (Berlioz, 1939)

Conothraupis speculigera (Gould, 1855)

Contopus cooperi (Nuttall, 1831)

Coryphaspiza melanotis (Temminck, 1822)

Coryphistera alaudina (Burmeister, 1860)

Cotinga maculata (Statius Muller, 1776)

Cranioleuca muelleri (Hellmayr, 1911)

Cranioleuca sulphurifera (Burmeister, 1869)

Crax blumenbachii (Spix, 1825)

Crax fasciolata pinima (Pelzeln, 1870)

Crax globulosa (Spix, 1825)

Crypturellus noctivagus (Wied, 1820)

Crypturellus noctivagus noctivagus (Wied, 1820)

Crypturellus noctivagus zabele (Spix, 1825)

Crypturellus strigulosus (Temminck, 1815)

Culicivora caudacuta (Vieillot, 1818)

Curaeus forbesi (Sclater, 1886)

Cyanocompsa brissonii (Lichtenstein, 1823)

Cyanocorax hafferi (Cohn-Haft, Santos-Júnior, Fernandes \& Ribas, 2013)

Cyanopsitta spixii (Wagler, 1832)

Dacnis nigripes (Pelzeln, 1856)

Dendrexetastes rufigula paraensis (Lorenz, 1895)

Dendrocincla fuliginosa trumaii (Sick, 1950)

Dendrocincla merula badia (Zimmer, 1934)

Dendrocincla taunayi (Pinto, 1939)

Dendrocolaptes medius (Todd, 1920)

Dendrocolaptes picumnus transfasciatus (Todd, 1925)

Dendrocolaptes retentus (Batista, Aleixo, Vallinoto, Azevedo, Rêgo, Silveira, Sampaio \& Schneider, 2013)

Dendrocolaptes ridgwayi (Hellmayr, 1905)

Diomedea dabbenena (Mathews, 1929)

Diomedea epomophora (Lesson, 1825)

Diomedea exulans (Linnaeus, 1758)

Diomedea sanfordi (Murphy, 1917)

Discosura (Popelaria) langsdorffi langsdorffi (Temminck, 1821)

Dixiphia pipra cephaleucos (Thunberg, 1822 )

Drymornis bridgesii (Eyton, 1849)

Dryocopus galeatus (Temminck, 1822)

Dysithamnus plumbeus (Wied, 1831)

Elaenia ridleyana (Sharpe, 1888)

\begin{tabular}{|c|c|c|}
\hline EN & EN & \\
\hline $\mathrm{RD}$ & EN & NA \\
\hline NT & NT & \\
\hline VU & VU & \\
\hline CR & CR & \\
\hline NT & LC & \\
\hline CR & $\mathrm{CR}$ & $\checkmark$ \\
\hline VU & EN & \\
\hline LC & VU & $\checkmark$ \\
\hline VU & VU & \\
\hline DD & EN & NA \\
\hline DD & NT & NA \\
\hline LC & NT & \\
\hline VU & EN & $\checkmark$ \\
\hline CR & $\mathrm{CR}$ & \\
\hline EN & CR & \\
\hline LC & VU & \\
\hline LC & NT & \\
\hline EN & CR & \\
\hline EN & $\mathrm{CR}$ & $\checkmark$ \\
\hline DD & EN & NA \\
\hline LC & NT & \\
\hline VU & VU & \\
\hline NT & VU & $\checkmark$ \\
\hline LC & NT & \\
\hline VU & LC & $\checkmark$ \\
\hline VU & VU & \\
\hline NT & LC & $\checkmark$ \\
\hline $\mathrm{RD}$ & VU & NA \\
\hline EN & CR & \\
\hline NT & LC & \\
\hline EN & EN & \\
\hline NA & NA & NA \\
\hline EN & VU & \\
\hline EN & EN & \\
\hline EN & VU & \\
\hline LC & $\mathrm{VU}$ & \\
\hline $\mathrm{RD}$ & $\mathrm{VU}$ & NA \\
\hline LC & NT & \\
\hline EN & CR & \\
\hline VU & VU & \\
\hline VU & CR & \\
\hline EN & EN & \\
\hline VU & EN & \\
\hline NT & LC & \\
\hline CR & NT & \\
\hline VU & EN & \\
\hline NT & EN & \\
\hline $\mathrm{VU}$ & VU & \\
\hline
\end{tabular}


Eleoscytalopus psychopompus (Teixeira \& Carnevalli, 1989)

Eucarthmus rufomarginatus (Pelzeln, 1868)

Formicivora erythronotos (Hartlaub, 1852)

Formicivora grantsaui (Gonzaga, Carvalhaes \& Buzzetti, 2007)

Formicivora iheringi (Hellmayr, 1909)

Formicivora littoralis (Gonzaga \& Pacheco, 1990)

Formicivora paludicola (Buzzetti, Belmontes-Lopes, Reinert, Silveira \& Bornschein, Fregata ariel (Gray, 1845)

Fregata minor (Gmelin, 1789)

Geositta poeciloptera (Wied, 1830)/ Geobates poecilopterus

Glaucis dohrnii (Bourcier \& Mulsant, 1852)

Grallaria varia distincta (Todd, 1927)

Grallaria varia intercedens (Berlepsch \& Leverkühn, 1890)

Guaruba guarouba (Gmelin, 1788)

Gubernatrix cristata (Vieillot, 1817)

Gygis alba (Sparmann, 1786)

Haematopus palliatus (Temminck, 1820)

Harpia harpyja (Linnaeus, 1758)

Hemitriccus furcatus (Lafresnaye, 1846)

Hemitriccus griseipectus (zosterops) naumburgae (Zimmer, 1945)

Hemitriccus kaempferi (Zimmer, 1953)

Hemitriccus mirandae (Snethlage, 1925)

Herpsilochmus pectoralis (Sclater, 1857)

Herpsilochmus pileatus (Lichtenstein, 1823)

Hydropsalis anomala (Gould, 1838)/ Eleothreptus anomalus

Hydropsalis (Caprimulgus) candicans (Pelzeln, 1867)

Hydropsalis vielliardi (Lencioni-Neto, 1994)

Hylexetastes brigidai (Silva, Novaes \& Oren, 1996)

Hylopezus ochroleucus (Wied, 1831)

Hylopezus paraensis (Snethlage, 1910)

Hylophilus ochraceiceps rubrifrons (Sclater \& Salvin, 1867)

Hypocnemis ochrogyna (Zimmer, 1932)

Ibycter americanus (Boddaert, 1783)

Iodopleura pipra (Lesson, 1831)

Iodopleura pipra leucopygia (Salvin, 1885)

Jacamaralcyon tridactyla (Vieillot, 1817)

Knipolegus franciscanus (Snethlage, 1928)

Laniisoma elegans (Thunberg, 1823)

Larus atlanticus (Olrog, 1958)

Lepidocolaptes wagleri (Spix, 1824)

Lepidothrix iris iris (Schinz, 1851)

Lepidothrix vilasboasi (Sick, 1959)

Leptasthenura platensis (Reichenbach, 1853)

Leptodon forbesi (Swann, 1922)

Limnoctites rectirostris (Gould, 1839)

Limnodromus griseus (Gmelin, 1789)

Lipaugus lanioides (Lesson, 1844)

Lophornis gouldii (Lesson, 1832)

Machaeropterus regulus (Hahn, 1819)

\begin{tabular}{|c|c|c|}
\hline LC & EN & \\
\hline NT & NT & \\
\hline EN & CR & \\
\hline $\mathrm{RD}$ & EN & $\mathrm{N}$ \\
\hline$N T$ & $N T$ & \\
\hline CR & EN & \\
\hline $\mathrm{RD}$ & CR & $\mathrm{N}$ \\
\hline CR & CR & \\
\hline CR & CR & \\
\hline VU & EN & \\
\hline EN & EN & \\
\hline LC & VU & \\
\hline VU & VU & \\
\hline VU & VU & \\
\hline CR & CR & \\
\hline NT & NT & \\
\hline LC & NT & \\
\hline NT & VU & $\nabla$ \\
\hline NT & $\mathrm{VU}$ & \\
\hline NT & $\mathrm{VU}$ & \\
\hline CR & VU & \\
\hline EN & VU & \\
\hline VU & LC & $\checkmark$ \\
\hline VU & VU & \\
\hline NT & NT & \\
\hline EN & VU & \\
\hline LC & NT & 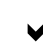 \\
\hline LC & $\mathrm{VU}$ & \\
\hline NT & NT & \\
\hline LC & $\mathrm{VU}$ & \\
\hline LC & VU & \\
\hline LC & $\mathrm{VU}$ & $v$ \\
\hline LC & NT & \\
\hline NT & EN & \\
\hline VU & CR & \\
\hline NT & NT & \\
\hline NT & NT & $v$ \\
\hline NT & NT & \\
\hline NA & NA & $\mathrm{N}$ \\
\hline VU & EN & $\checkmark$ \\
\hline LC & EN & \\
\hline DD & VU & $\mathrm{N}$ \\
\hline CR & CR & \\
\hline DD & EN & $\mathrm{N}$ \\
\hline VU & NT & \\
\hline LC & CR & \\
\hline NT & NT & \\
\hline LC & VU & \\
\hline NT & LC & \\
\hline
\end{tabular}


Mergus octosetaceus (Vieillot, 1817)

Merulaxis stresemanni (Sick, 1960)

Micrastur mintoni (Whittaker, 2002)

Micropygia schomburgkii (Cabanis, 1848)

Mimus gilvus antelius (Oberholser, 1919)

Mitu (Pauxi) mitu (Linnaeus, 1766)

Momotus momota marcgraviana (Pinto \& Camargo, 1961)

Monasa morphoeus morphoeus (Hahn \& Küster, 1823)

Morphnus guianensis (Daudin, 1800)

Myrmeciza (Myrmoderus) ruficaudus (Wied, 1831)

Myrmotherula fluminensis (Gonzaga, 1988)

Myrmotherula klagesi (Todd, 1927)

Myrmotherula minor (Salvadori, 1864)

Myrmotherula snowi (Teixeira \& Gonzaga, 1985)

Myrmotherula unicolor (Ménétriès, 1835)

Myrmotherula urosticta (Sclater, 1857)

Nemosia rourei (Cabanis, 1870)

Neomorphus geoffroyi amazonicus (Pinto, 1964)

Neomorphus geoffroyi dulcis (Snethlage, 1927)

Neomorphus geoffroyi geoffroyi (Temminck, 1820)

Neomorphus squamiger (Todd, 1925)

Neopelma aurifrons (Wied, 1831)

Nyctipolus hirundinaceus vieilliardi (Ribon, 1995)

Notharchus swainsoni (Gray, 1846)

Nothura minor (Spix, 1825)

Numenius hudsonicus (Latham, 1790)

Nyctibius aethereus aethereus (Wied, 1820)

Nyctibius leucopterus (Wied, 1821)

Nystalus torridus (Bond \& Meyer de Schauensee, 1940)

Odontophorus capueira plumbeicollis (Cory, 1915)

Onychorhynchus swainsoni (Pelzeln, 1858)

Ortalis guttata remota (Pinto, 1960)

Penelope jacucaca (Spix, 1825)

Penelope ochrogaster (Pelzeln, 1870)

Penelope pileata (Wagler, 1830)

Penelope superciliaris alagoensis (Nardelli, 1993)

Phaethon aethereus (Linnaeus, 1758)

Phaethon lepturus (Daudin, 1802)

Phaethornis aethopygus (Zimmer, 1950)

Phaethornis bourcieri major (Hinkelmann, 1989)

Phaethornis margarettae camargoi (Grantsau, 1988)

Phaethornis margarettae (Ruschi, 1972)

Philydor novaesi (Teixeira \& Gonzaga, 1983)

Phlegopsis nigromaculata confinis (Zimmer, 1932)

Phlegopsis nigromaculata paraensis (Hellmayr, 1904)

Phylloscartes beckeri (Gonzaga \& Pacheco, 1995)

Phylloscartes ceciliae (Teixeira, 1987)

Phylloscartes eximius (Temminck, 1822)

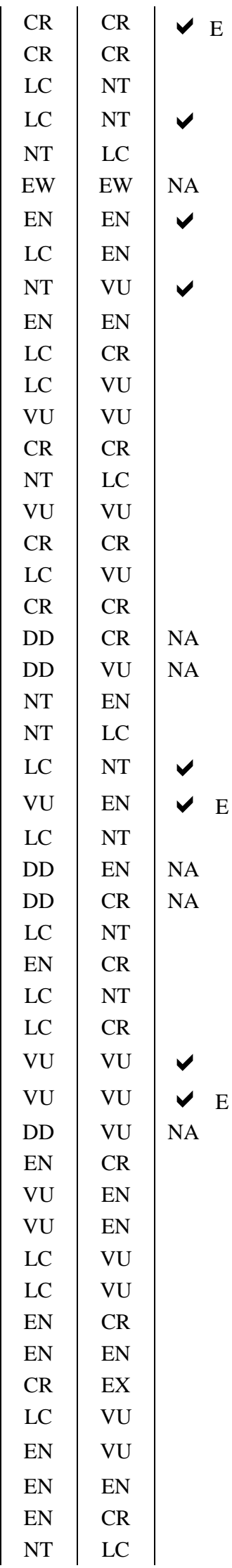


Phylloscartes kronei (Willis \& Oniki, 1992)

Phylloscartes paulista (Ihering \& Ihering, 1907)

Phylloscartes roquettei (Snethlage, 1928)

Phylloscartes sylviolus (Cabanis \& Heine, 1859)

Piculus paraensis (Snethlage, 1907)

Piculus polyzonus (Valenciennes, 1826)

Picumnus exilis (pernambucensis) (Zimmer, 1947)

Picumnus fuscus (Pelzeln, 1870)

Picumnus limae (Snethlage, 1924)

Picumnus varzeae (Snethlage, 1912)

Pionus reichenowi (Heine, 1844)

Piprites chloris grisescens (Novaes, 1964)

Piprites pileatus (pileata) (Temminck, 1822)

Platyrinchus leucoryphus (Wied, 1831)

Platyrinchus mystaceus niveigularis (Pinto, 1954)

Polioptila lactea (Sharpe, 1885)

Polystictus pectoralis (Vieillot, 1817)

Polystictus pectoralis brevipennis (Berlepsch \& Hartert, 1902)

Porzana spiloptera (Durnford, 1877)

Primolius maracana (Vieillot, 1816)

Procellaria aequinoctialis (Linnaeus, 1758)

Procellaria conspicillata (Gould, 1844)

Procnias albus wallacei (Oren \& Novaes, 1985)

Procnias averano (averano) (Hermann, 1783)

Procnias nudicollis (Vieillot, 1817)

Pseudastur polionotus (Kaup, 1847)

Pseudoseisura lophotes (Reichenbach, 1853)

Psophia dextralis (Conover, 1934)

Psophia interjecta (Griscom \& Greenway, 1937)

Psophia obscura (Pelzeln, 1857)

Psophia viridis (Spix, 1825)

Pterodroma arminjoniana (Giglioli \& Salvatori, 1869)

Pterodroma deserta (Mathews, 1934)

Pterodroma incerta (Schlegel, 1863)

Pterodroma madeira (Mathews, 1934)

Pteroglossus bailloni (Vieillot, 1819)

Pteroglossus bitorquatus bitorquatus (Vigors, 1826)

Puffinus lherminieri (Lesson, 1839)

Pulsatrix perspicillata pulsatrix (Wied, 1820)

Pygochelidon melanoleuca (Wied, 1820)

Pyriglena atra (Swainson, 1825)

Pyriglena pernambucensis (Zimmer,1931)

Pyrilia vulturina (Kuhl, 1820)

Pyroderus scutatus (Shaw, 1792)

Pyrrhura anaca (griseipectus) (Gmelin, 1788)

Pyrrhura cruentata (Wied, 1820)

Pyrrhura devillei (Massena \& Souancé, 1854)

Pyrrhura griseipectus (Salvadori, 1900)

Pyrrhura lepida anerythra (Neumann, 1927)

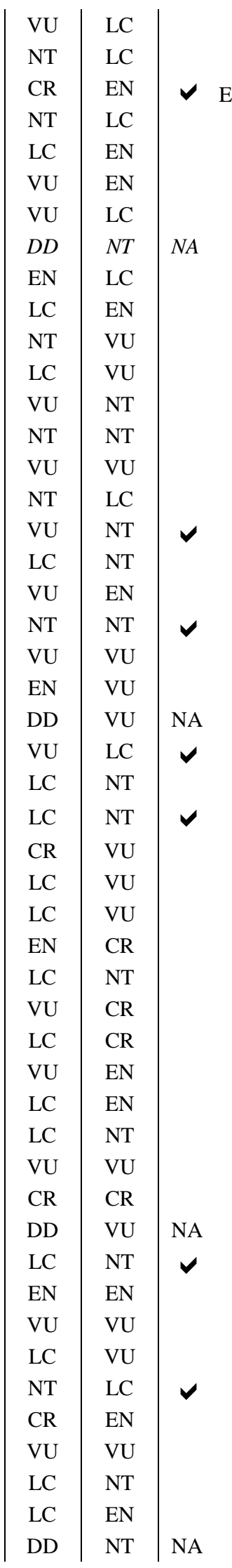


Pyrrhura lepida coerulescens (Neumann, 1927)

Pyrrhura lepida lepida (Wagler, 1832)

Pyrrhura leucotis (Kuhl, 1820)

Pyrrhura molinae (Massena \& Souancé, 1854)

Pyrrhura pfrimeri Miranda-Ribeiro, 1920

Rhegmatorhina gymnops (Ridgway, 1888)

Rhopornis ardesiacus (Wied, 1831)

Sarcoramphus papa (Linnaeus, 1758)

Schiffornis turdina intermedia (Pinto, 1954)

Sclerurus caudacutus caligineus (Pinto, 1954)

Sclerurus caudacutus umbretta (Lichtenstein, 1823)

Sclerurus cearensis (Snethlage, 1924)

Sclerurus macconnelli bahiae (Chubb, 1919)

Scytalopus diamantinensis (Bornschein, Maurício, Belmonte-Lopes, Mata \& Bonato, 2007)

Scytalopus gonzagai (Maurício, Belmonte-Lopes, Pacheco, Silveira, Whitney \& Bornschein 2014)

Scytalopus iraiensis (Bornschein, Reinert \& Pichorim, 1998)

Scytalopus novacapitalis (Sick, 1958)

Selenidera gouldii baturitensis (Pinto \& Camargo 1961)

Serpophaga hypoleuca pallida (Snethlage, 1907)

Spartonoica maluroides (d'Orbigny \& Lafresnaye, 1837)

Spheniscus magellanicus (Forster, 1781)

Spizaetus ornatus (Daudin, 1800)

Spizaetus tyrannus (Wied, 1820)

Sporagra yarrellii (Audubon, 1839)

Sporophila beltoni (Repenning \& Fontana, 2013)

Sporophila cinnamomea (Lafresnaye, 1839)

Sporophila falcirostris (Temminck, 1820)

Sporophila frontalis (Verreaux, 1869)

Sporophila hypoxantha (Cabanis, 1851)

Sporophila maximiliani (Cabanis, 1851)

Sporophila melanogaster (Pelzeln, 1870)

Sporophila nigrorufa (d'Orbigny \& Lafresnaye, 1837)

Sporophila palustris (Barrows, 1883)

Sporophila pileata (Sclater, 1864)

Sporophila ruficollis (Cabanis, 1851)

Sterna dougallii (Montagu, 1813)

Sterna hirundinacea (Lesson, 1831)

Stigmatura napensis napensis (Chapman, 1926)

Strix huhula albomarginata (Spix, 1824)

Stymphalornis acutirostris (Bornschein, Reinert \& Teixeira, 1995)

Sula sula (Linnaeus, 1766)

Synallaxis cinerea (whitneyi) (Wied, 1831)

Synallaxis infuscata (Pinto, 1950)

Synallaxis kollari (Pelzeln, 1856)

Synallaxis rutilans omissa (Hartert, 1901)

Synallaxis simoni (Hellmayr, 1907)

Thalasseus (Sterna)sandvicensis eurygnatha (Latham, 1787)

Tangara cyanocephala (corallina) (Berlepsch, 1903)

\begin{tabular}{|c|c|c|}
\hline EN & NA & NA \\
\hline EN & VU & $\checkmark$ \\
\hline VU & VU & \\
\hline LC & NT & $\checkmark$ \\
\hline VU & EN & $\checkmark \mathrm{E}$ \\
\hline LC & VU & \\
\hline EN & EN & \\
\hline LC & NT & $\checkmark$ \\
\hline VU & VU & \\
\hline EN & CR & \\
\hline EN & $\mathrm{CR}$ & \\
\hline VU & VU & \\
\hline LC & VU & \\
\hline $\mathrm{RD}$ & EN & NA \\
\hline $\mathrm{RD}$ & EN & NA \\
\hline EN & EN & \\
\hline NT & EN & $\checkmark \mathrm{E}$ \\
\hline LC & EN & \\
\hline LC & VU & \\
\hline NT & LC & \\
\hline LC & NT & \\
\hline LC & NT & $\checkmark$ \\
\hline NT & LC & $\checkmark$ \\
\hline LC & VU & $\checkmark$ \\
\hline $\mathrm{RD}$ & VU & NA \\
\hline EN & NT & $\checkmark$ \\
\hline VU & VU & \\
\hline VU & VU & \\
\hline DD & VU & NA \\
\hline CR & CR & $\checkmark$ \\
\hline VU & VU & $\checkmark$ \\
\hline VU & VU & $\checkmark$ \\
\hline EN & VU & $\checkmark$ \\
\hline LC & NT & $\checkmark$ \\
\hline DD & VU & NA \\
\hline DD & VU & NA \\
\hline LC & VU & \\
\hline LC & VU & $\checkmark$ \\
\hline LC & VU & \\
\hline EN & EN & \\
\hline NT & EN & \\
\hline VU & NT & \\
\hline EN & EN & \\
\hline DD & EN & NA \\
\hline LC & NT & \\
\hline VU & LC & $\checkmark \mathrm{E}$ \\
\hline NT & LC & \\
\hline VU & LC & \\
\hline
\end{tabular}


Tangara cyanocephala cearensis (Cory, 1916)

Tangara fastuosa (Lesson, 1831)

Tangara mexicana (Linnaeus, 1766)

Tangara peruviana (Desmarest, 1806)

Tangara velia signata (Hellmayr, 1905)

Taoniscus nanus (Temminck, 1815)

Terenura sicki (Teixeira \& Gonzaga, 1983)

Thalassarche chlororhynchos (Gmelin, 1789)

Thalassarche melanophris (Temminck, 1828)

Thalasseus maximus (Boddaert, 1783)

Thalurania watertonii (Bourcier, 1847)

Thamnomanes caesius caesius (Temminck, 1820)

Thamnophilus aethiops distans (Pinto, 1954)

Thamnophilus caerulescens cearensis (Cory, 1919)

Thamnophilus caerulescens pernambucensis (Naumburg, 1937)

Thamnophilus nigrocinereus (Sclater, 1855)

Thamnophilus nigrocinereus tschudii (Pelzeln, 1868)

Thripophaga macroura (Wied, 1821)

Tigrisoma fasciatum (Such, 1825)

Tijuca condita (Snow, 1980)

Tinamus solitarius (Vieillot, 1819)

Tinamus tao (Temminck, 1815)

Touit melanonotus (Wied, 1820)

Touit surdus (Kuhl, 1820)

Triclaria malachitacea (Spix, 1824)

Trogon collaris eytoni (Fraser, 1837)

Urubitinga coronata (Vieillot, 1817)/ Harpyhaliaetus coronatus

Vireo gracilirostris (Sharpe, 1890)

Xanthopsar flavus (Gmelin, 1788)

Xenops minutus alagoanus (Pinto, 1954)

Xiphocolaptes carajaensis (Silva, Novaes \& Oren, 2002)

Xiphocolaptes falcirostris (Spix, 1824)

Xipholena atropurpurea (Wied, 1820)

Xiphorhynchus atlanticus (Cory, 1916)

Xiphorhynchus guttatus gracilirostris (Pinto \& Camargo 1957)

Xolmis dominicanus (Vieillot, 1823)

\begin{tabular}{|l|l|l|} 
NA & VU & NA \\
VU & VU & \\
NT & LC & \\
DD & VU & NA \\
NT & VU & \\
VU & EN & $\checkmark$ E \\
EN & CR & \\
VU & EN & \\
VU & NT & \\
VU & EN & \\
VU & EN & \\
LC & VU & \\
EN & EN & \\
EN & VU & \\
VU & VU & \\
LC & NT & \\
LC & EN & \\
VU & VU & \\
EN & VU & $\checkmark$ \\
VU & VU & \\
NT & NT & \\
LC & VU & $\checkmark$ \\
VU & VU & \\
NT & VU & \\
NT & LC & \\
LC & EN & \\
VU & EN & $\checkmark$ \\
VU & VU & \\
VU & VU & \\
VU & VU & \\
LC & VU & \\
VU & VU & $\checkmark$ \\
EN & VU & \\
VU & VU & \\
LC & EN & \\
NT & VU & \\
& & \\
&
\end{tabular}


Tabela S6: Compilação sobre os registros das espécies-alvo: Treino e Teste; análise de sensitividade dos modelos binários: presença, (omissão) e razão (\%); resultados para o Teste ROC Parcial ou Teste T (com asteriscos).

\begin{tabular}{|c|c|c|c|c|c|}
\hline & ESPÉCIE & $\begin{array}{l}\text { REGISTROS } \\
\text { TREINO } \\
\text { (N.) }\end{array}$ & $\begin{array}{c}\text { ANÁLISE DE } \\
\text { SENSITIVIDADE }\end{array}$ & $\begin{array}{l}\text { REGISTROS } \\
\text { TESTE } \\
\text { (N.) }\end{array}$ & $\begin{array}{c}\text { ROC-PARCIAL } \\
\text { (TESTE T) } \\
* * *(P<0,001)\end{array}$ \\
\hline \multirow{17}{*}{$\sum_{\forall}^{\infty}$} & Alectrurus tricolor & 60 & $25(1) 4 \%$ & 25 & $1.055813 * * *$ \\
\hline & Aliopsitta xanthops & 222 & 55 (8) $14,54 \%$ & 55 & $1.001487 * * *$ \\
\hline & Anodorhynchus hyacinthinus & 106 & 26 (2) $7,69 \%$ & 26 & $1.023017 * * *$ \\
\hline & Asthenes luizae & 24 & $6(1) 16,7 \%$ & 6 & $1.316346 * * *$ \\
\hline & Hydropsalis candicans & 26 & 6 (2) $33,34 \%$ & 6 & $1.109047 * * *$ \\
\hline & Celeus obrieni & 58 & 14 (1) $7,14 \%$ & 14 & $1.178917 * * *$ \\
\hline & Cercomacra ferdinandi & 77 & 19 (5) $26,3 \%$ & 19 & $1.012195 * * *$ \\
\hline & Columbina cyanopis & 16 & 4 (1) $25 \%$ & 4 & $1.359195 * * *$ \\
\hline & Conothraupis mesoleuca & 12 & $3(0) 0 \%$ & 3 & $1.012195 * * *$ \\
\hline & Euscarthmus rufomarginatus & 47 & 17 (2) $11,76 \%$ & 17 & $1.000445 * * *$ \\
\hline & Geositta poeciloptera & 79 & 20 (1) $5 \%$ & 20 & $1.841514 * * *$ \\
\hline & Nothura minor & 114 & 29 (7) $24,14 \% \%$ & 29 & $1.008803 * * *$ \\
\hline & Penelope ochrogaster & 101 & $25(2) 8 \%$ & 25 & $1.074651 * * *$ \\
\hline & Phylloscartes roquettei & 31 & $10(2) 20 \%$ & 10 & $1.055525 * * *$ \\
\hline & Pyrrhura pfrimeri & 37 & 9 (2) $22,23 \%$ & 9 & $1.364952 * * *$ \\
\hline & Scytalopus novacapitalis & 50 & $12(0) 0 \%$ & 12 & $1.350366 * * *$ \\
\hline & Taoniscus nanus & 135 & 34 (6) $17,64 \% \%$ & 34 & $1.030188 * * *$ \\
\hline \multirow{13}{*}{$\frac{\sqrt{2}}{2}$} & Kerodon acrobata & 11 & PMC & - & - \\
\hline & Lonchophylla bokermanni & 7 & $2(0) 0 \%$ & 2 & 1.943401 \\
\hline & Lycalopex vetulus & 29 & 7 (1) $14,28 \%$ & 7 & $1.056845 * * *$ \\
\hline & Thalpomys cerradensis & 18 & $5(1) 20 \%$ & 5 & $1.112077 * * *$ \\
\hline & Thalpomys lasiotis & 16 & $4(0) 0 \%$ & 4 & $1.473239 * * *$ \\
\hline & Thylamys velutinus & 7 & $2(0) 0 \%$ & 2 & 1.448347 \\
\hline & Oligoryzomys rupestres & 4 & PMC & - & - \\
\hline & Euryoryzomys lamia & 5 & PMC & - & - \\
\hline & Phyllomys brasiliensis & 3 & PMC & - & - \\
\hline & Glyphonycteris behnii & 7 & PMC & - & - \\
\hline & Microakodontomys transitorius & 2 & PMC & - & - \\
\hline & Juscelinomys candango & 2 & & - & - \\
\hline & Gyldenstolpia planaltensis & 4 & PMC & - & - \\
\hline \multirow{4}{*}{ ANFÍBIOS } & Phyllomedusa centralis & 6 & $2(0) 0 \%$ & 2 & 1.506341 \\
\hline & Proceratophrys moratoi & 7 & $2(0) 0 \%$ & 2 & 1.5583068 \\
\hline & Bokermannohyla martinsi & 26 & $6(0) 0 \%$ & 6 & 1.9508102 \\
\hline & Allobates goianus & 4 & PMC & - & - \\
\hline \multirow{9}{*}{ 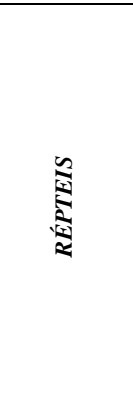 } & Bothrops itapetiningae & 145 & $36(8) 22,23 \%$ & 36 & $1.024103 * * *$ \\
\hline & Heterodactylus lundii & 8 & $2(0) 0 \%$ & 2 & $1.424235 * * *$ \\
\hline & Kentropyx vanzoi & 22 & $6(0) 0 \%$ & 6 & $1.027119 * * *$ \\
\hline & Phalotris lativittatus & 38 & $9(0) 0 \%$ & 9 & $1.317787 * * *$ \\
\hline & Philodryas livida & 22 & $5(0) 0 \%$ & 5 & $1.426472 * * *$ \\
\hline & Placosoma cipoense & 5 & $1(0) 0 \%$ & 1 & $1.317787 * * *$ \\
\hline & Hydrodynastes melanogigas & 7 & PMC & - & - \\
\hline & Ameiva parecis & 2 & - & - & - \\
\hline & Bachia psamophila & 1 & - & - & - \\
\hline
\end{tabular}




\begin{tabular}{|l|l|r|r|r|r|}
\hline \multicolumn{1}{|c|}{ ESPÉCIE } & $\begin{array}{c}\text { REGISTROS } \\
\text { TREINO } \\
\text { (N.) }\end{array}$ & $\begin{array}{c}\text { ANÁLISE DE } \\
\text { SENSITIVIDADE }\end{array}$ & $\begin{array}{c}\text { REGISTROS } \\
\text { TESTE } \\
\text { (N.) }\end{array}$ & $\begin{array}{c}\text { ROC-PARCIAL } \\
\text { (TESTE T) } \\
* * * *(P<0,001)\end{array}$ \\
\hline \multirow{2}{*}{} & Liotyphlops schubarti & 2 & - & - & - \\
\cline { 2 - 5 } & Apostolepis striata & 2 & - & - & - \\
Bachia didactyla & 5 & PMC & - & - \\
& Apostolepis serrana & 2 & - & - & - \\
& Phalotris_multipunctatus & 3 & - & - & - \\
\hline
\end{tabular}
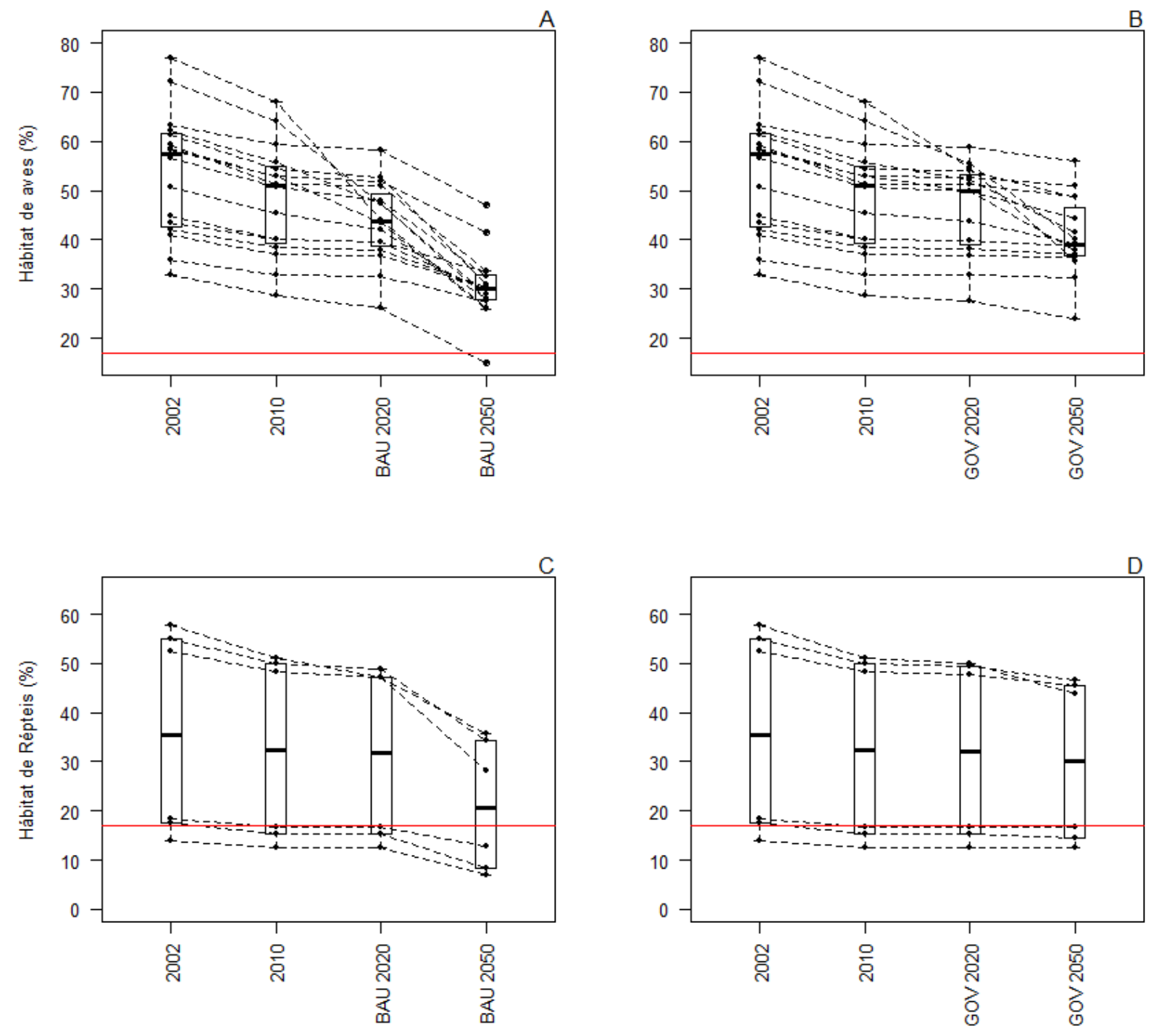

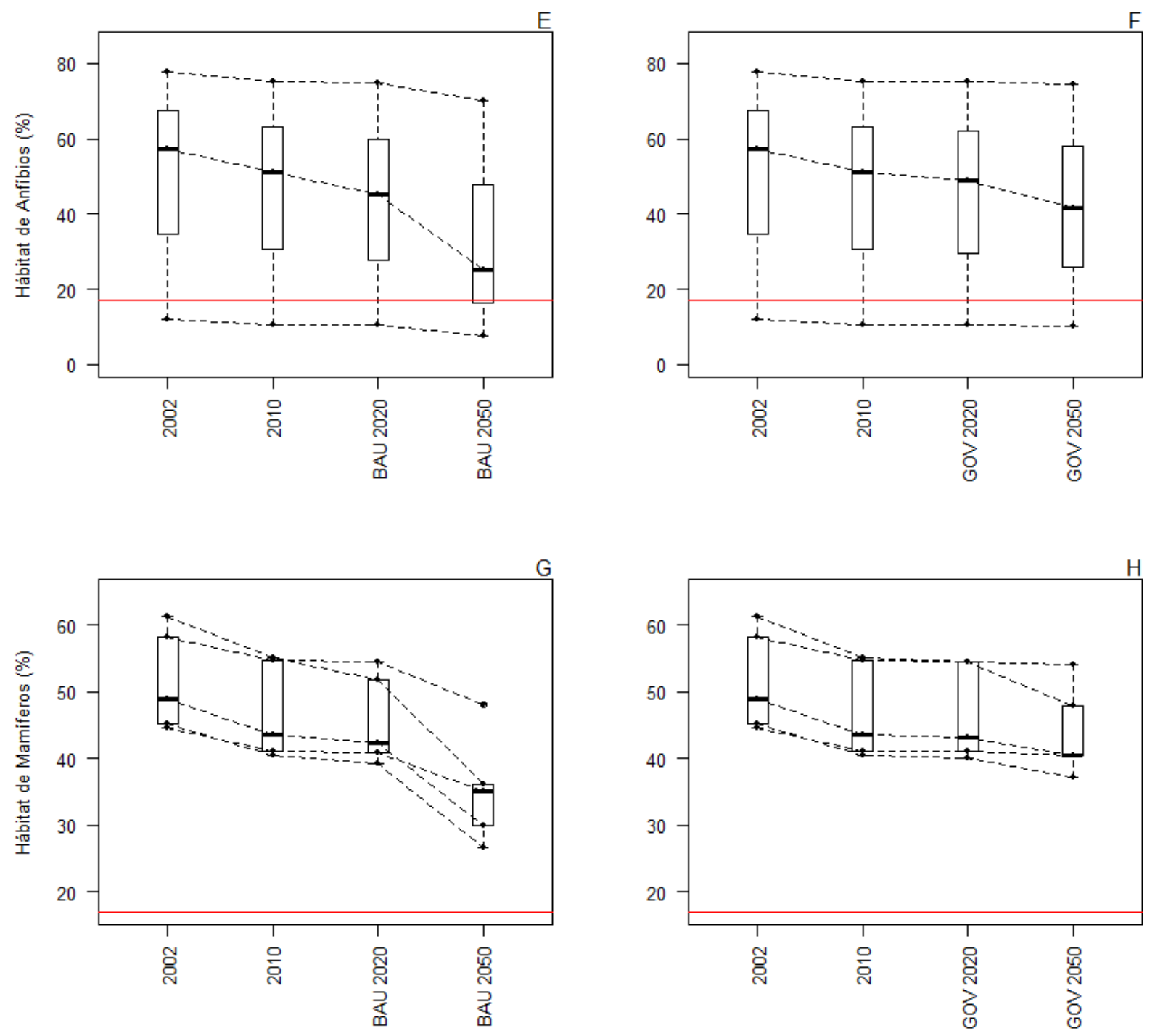

Figura S1: Remanescentes dos SDMs, anos de 2002 e 2010 e os cenários BAU 2010 - 2020 e BAU 2010 - 2050 e os cenários GOV. 2010 - 2020 e GOV. 2010 - 2050, A e B, espécies de aves; C e D, espécies de répteis; E e F, espécies de anfíbios e, $\mathrm{G}$ e $\mathrm{H}$, espécies de mamíferos. 


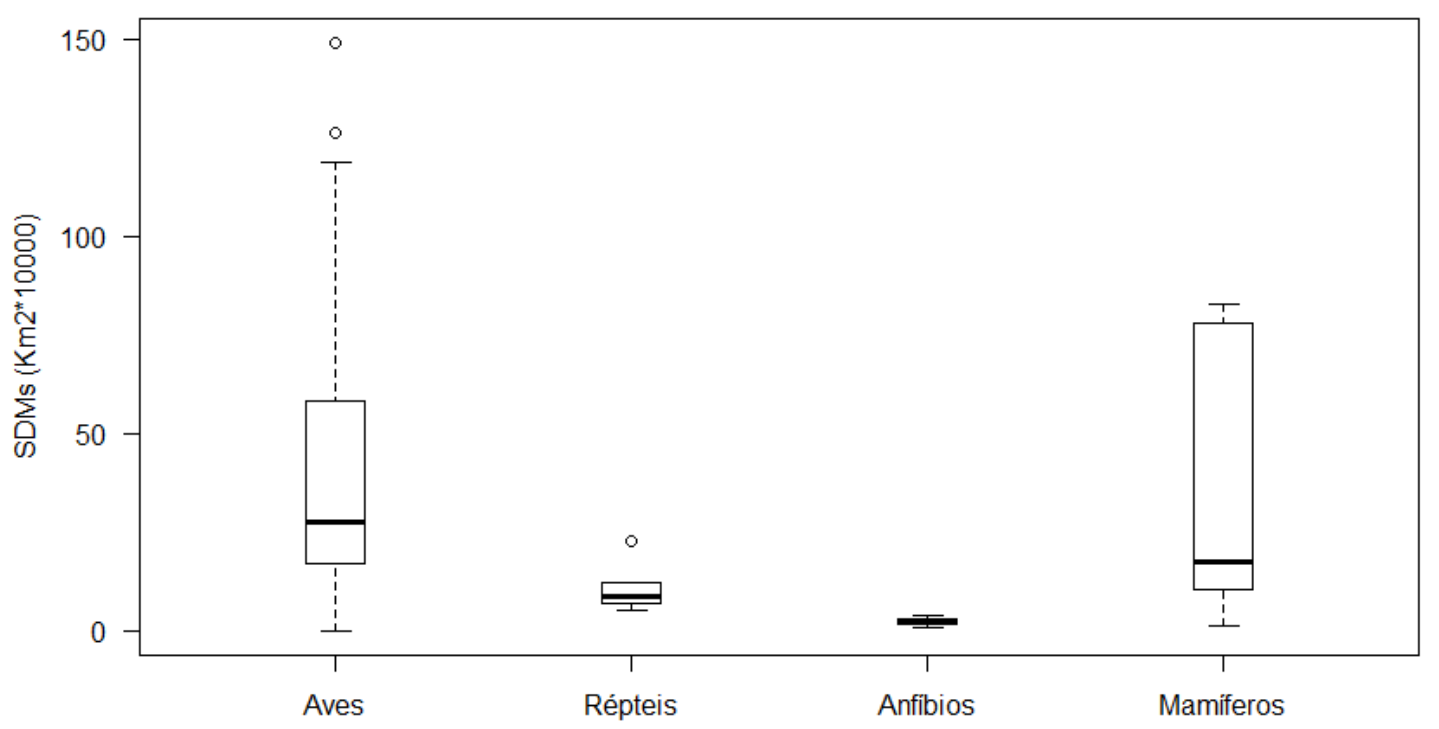

Figura S2: Área média dos SDMs por táxon. 
Tabela S7: Cálculo do SDMs das espécies-alvo (SDMs original) e, porcentagem remanescente, descontando as áreas desmatadas em 2002; 2010; cenários BAU 2010 - 2020 e 2010 - 2050, e cenários GOV. 2010 - 2020 e 2010 - 2050

\begin{tabular}{|c|c|c|c|c|c|c|c|c|}
\hline \multicolumn{2}{|r|}{ ESPÉCIE } & $\begin{array}{l}\text { SDMs Orig. } \\
\left(\mathrm{Km}^{2}\right)\end{array}$ & $2002(\%)$ & $2010(\%)$ & BAU $2020(\%)$ & BAU $2050(\%)$ & GOV. $2020(\%)$ & GOV. $2050(\%)$ \\
\hline \multirow{17}{*}{ 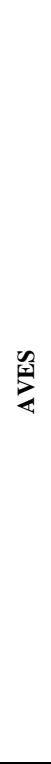 } & Alectrurus tricolor (Vieillot, 1816) & 509145.5 & 40.87 & 36.96 & 36.77 & 30.45 & 36.88 & 36.44 \\
\hline & Alipiopsitta xanthops (Spix, 1824) & 1261165 & 50.77 & 45.34 & 42.12 & 28.16 & 43.75 & 39.29 \\
\hline & Anodorhynchus hyacinthinus (Latham, 1790) & 1187549.75 & 62.16 & 55.61 & 47.46 & 28.85 & 52.02 & 41.55 \\
\hline & Asthenes luizae (Vielliard, 1990) & 30119 & 63.20 & 59.41 & 58.22 & 47.12 & 58.95 & 56.04 \\
\hline & Celeus obrieni (Short, 1973) & 582120 & 72.16 & 64.20 & 47.47 & 28.07 & 55.55 & 40.00 \\
\hline & Cercomacra ferdinandi (Snethlage, 1928) & 397213 & 76.88 & 67.95 & 43.89 & 26.22 & 54.51 & 35.63 \\
\hline & Columbina cyanopis (Pelzeln, 1870) & 249189.75 & 32.78 & 28.68 & 26.27 & 15.03 & 27.41 & 24.04 \\
\hline & Conothraupis mesoleuca (Berlioz, 1939) & 169350 & 58.27 & 52.95 & 51.96 & 41.62 & 52.72 & 51.05 \\
\hline & Euscarthmus rufomarginatus (Pelzeln, 1868) & 1490587.5 & 56.62 & 50.70 & 47.88 & 32.50 & 50.00 & 44.37 \\
\hline & Geositta poeciloptera (Wied, 1830) & 751057.5 & 44.93 & 40.15 & 39.52 & 29.88 & 39.79 & 38.60 \\
\hline & Hydropsalis candicans (Pelzeln, 1867) & & & & & & & \\
\hline & Nothura minor (Spix, 1825) & 238926.5 & 35.83 & 32.78 & 32.63 & 27.59 & 32.71 & 32.31 \\
\hline & Penelope ochrogaster (Pelzeln, 1870) & 277930.75 & 58.57 & 52.87 & 43.59 & 25.87 & 49.78 & 37.80 \\
\hline & Phylloscartes roquettei (Snethlage, 1928) & 142241.25 & 61.28 & 54.38 & 52.66 & 30.79 & 53.95 & 48.72 \\
\hline & Pyrrhura pfrimeri (Miranda-Ribeiro, 1920) & 85098.75 & 59.31 & 51.31 & 50.86 & 33.69 & 51.20 & 48.77 \\
\hline & Scytalopus novacapitalis (Sick, 1958) & 171605.25 & 43.47 & 40.05 & 39.57 & 33.35 & 39.83 & 38.80 \\
\hline & Taoniscus nanus (Temminck, 1815) & 332441.25 & 42.05 & 38.44 & 37.93 & 30.45 & 38.26 & 37.06 \\
\hline \multirow{6}{*}{ 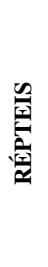 } & Bothrops itapetiningae (Boulenger, 1907) & 97503.25 & 18.45 & 16.75 & 16.71 & 12.93 & 16.71 & 16.68 \\
\hline & Heterodactylus lundii (Reinhardt \& Lütken, 1862) & 67829.25 & 52.48 & 48.21 & 47.13 & 35.84 & 47.70 & 45.39 \\
\hline & Kentropyx vanzoi (Gallagher \& Dixon, 1980) & 226639.25 & 57.80 & 51.20 & 47.30 & 28.26 & 49.88 & 43.78 \\
\hline & Phalotris lativittatus (Ferrarezzi, 1993) & 50314.75 & 13.99 & 12.47 & 12.45 & 6.90 & 12.45 & 12.43 \\
\hline & Philodryas livida (Amaral, 1923) & 120931.75 & 17.67 & 15.41 & 15.24 & 8.44 & 15.32 & 14.60 \\
\hline & Placosoma cipoense (Cunha, 1966) & 74154.5 & 54.90 & 49.84 & 48.76 & 34.39 & 49.38 & 46.67 \\
\hline \multirow{3}{*}{ 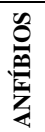 } & Phyllomedusa centralis (Bokermann, 1965) & 37173 & 57.41 & 51.17 & 45.18 & 25.22 & 48.88 & 41.62 \\
\hline & Proceratophrys moratoi (Jim \& Caramaschi, 1980) & 21964.75 & 12.03 & 10.42 & 10.38 & 7.70 & 10.38 & 10.37 \\
\hline & Bokermannohyla martinsi (Bokermann, 1964) & 9050.75 & 77.89 & 75.27 & 74.88 & 70.25 & 75.04 & 74.35 \\
\hline \multirow{5}{*}{ 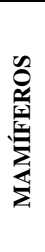 } & $\begin{array}{l}\text { Lonchophylla bokermanni (Sazima, Vizotto \& Taddei, } \\
\text { 1978) }\end{array}$ & 12576.5 & 58.19 & 54.68 & 54.51 & 48.07 & 54.58 & 54.10 \\
\hline & Lycalopex vetulus (Lund, 1842) & 780485 & 45.21 & 40.47 & 39.16 & 26.64 & 40.02 & 37.21 \\
\hline & Thalpomys cerradensis (Hershkovitz, 1990) & 105910.75 & 61.33 & 55.23 & 51.75 & 36.13 & 54.58 & 47.97 \\
\hline & Thalpomys lasiotis (Thomas, 1916) & 175149 & 44.69 & 41.20 & 40.97 & 35.10 & 41.11 & 40.39 \\
\hline & Thylamys velutinus (Wagner, 1842) & 829617.5 & 48.86 & 43.68 & 42.30 & 29.98 & 43.20 & 40.50 \\
\hline
\end{tabular}



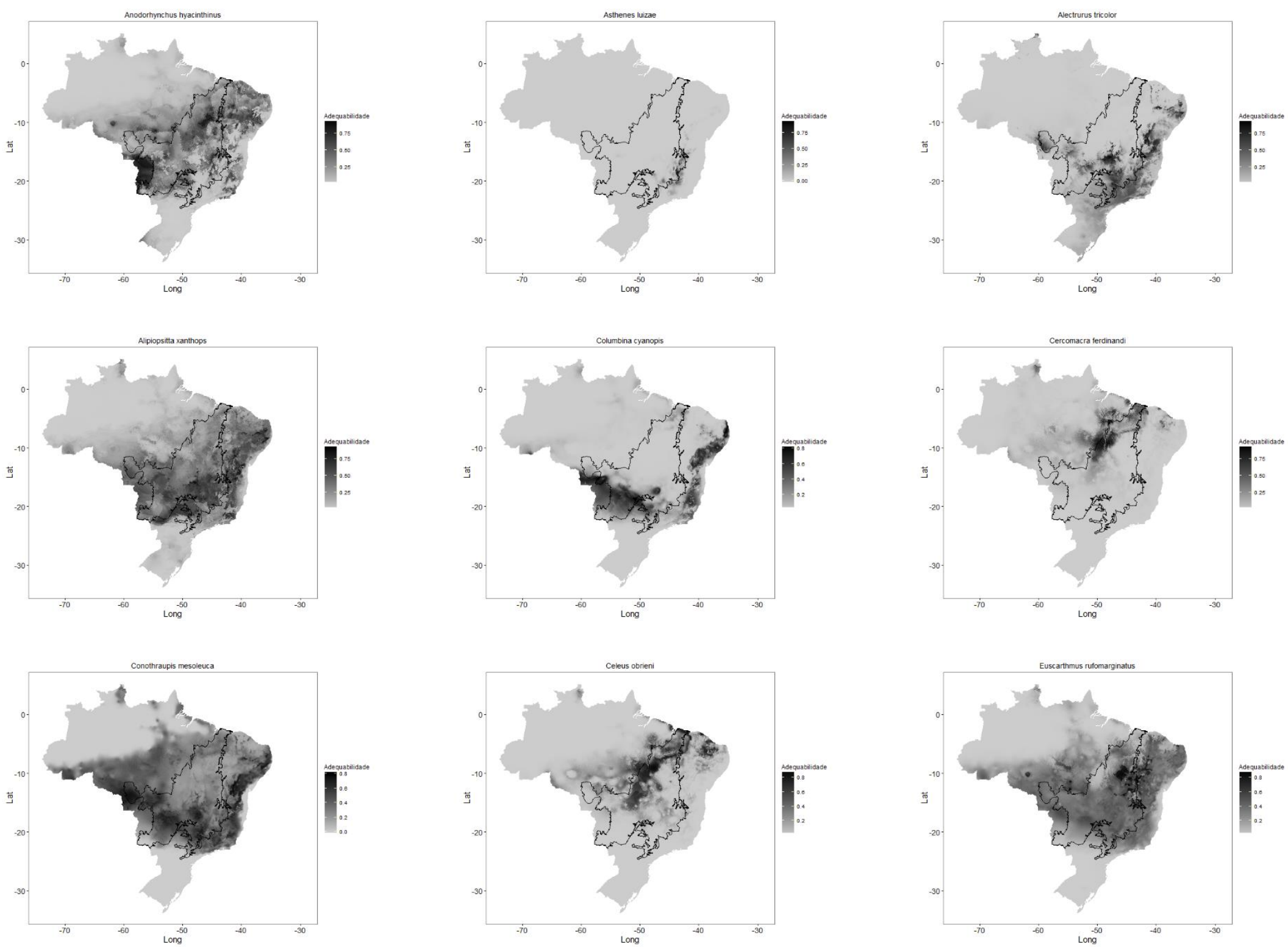

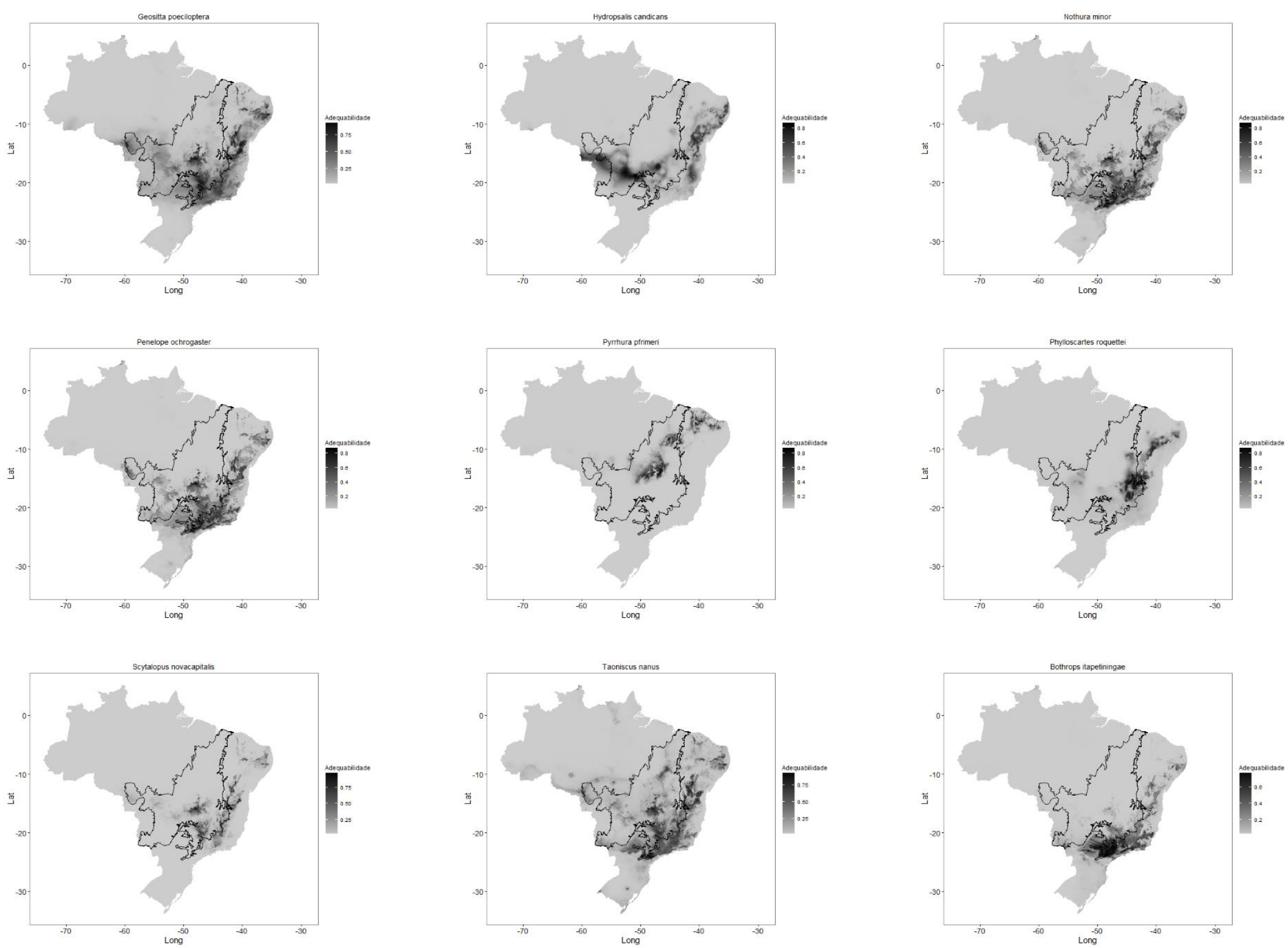

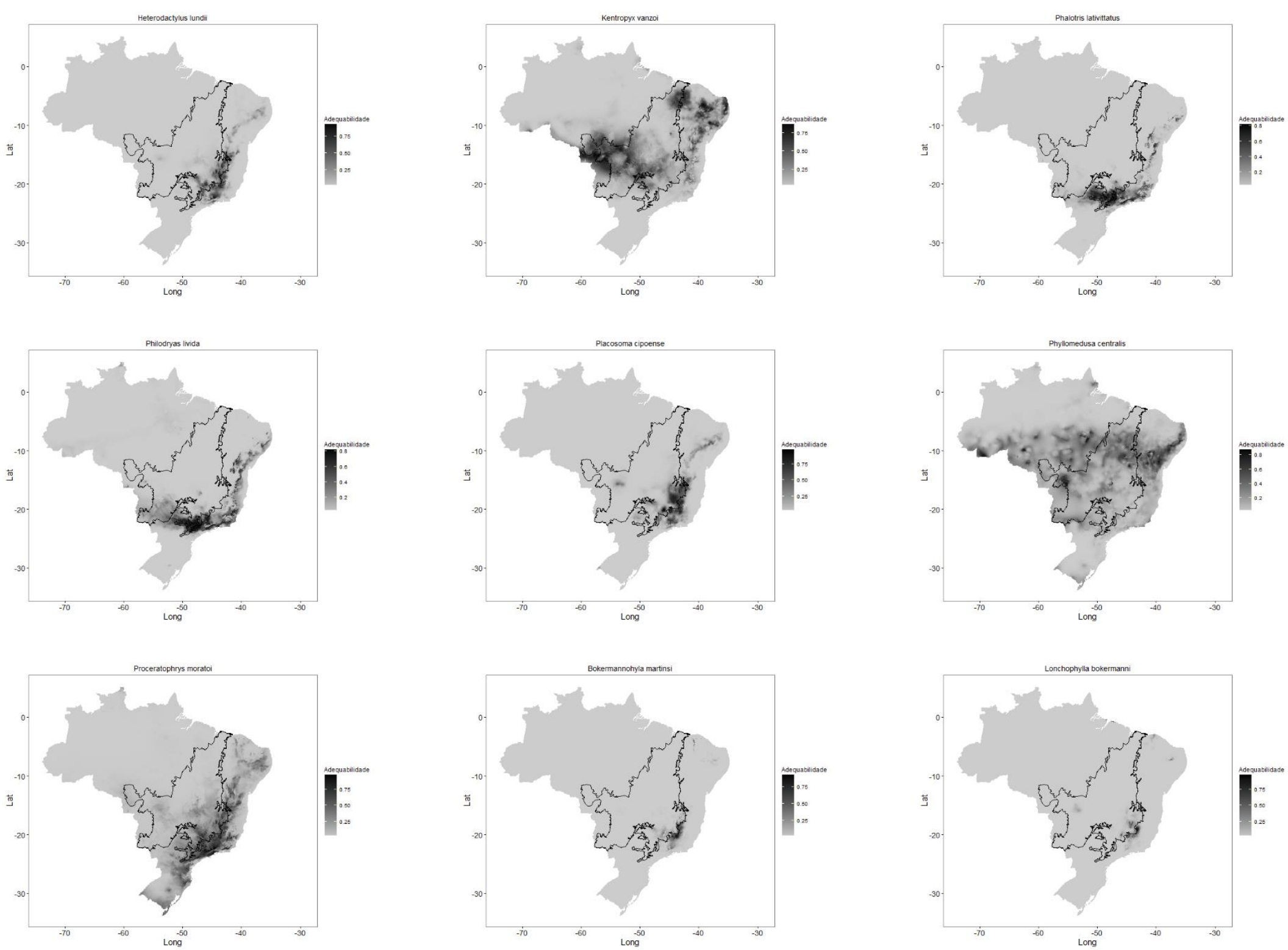

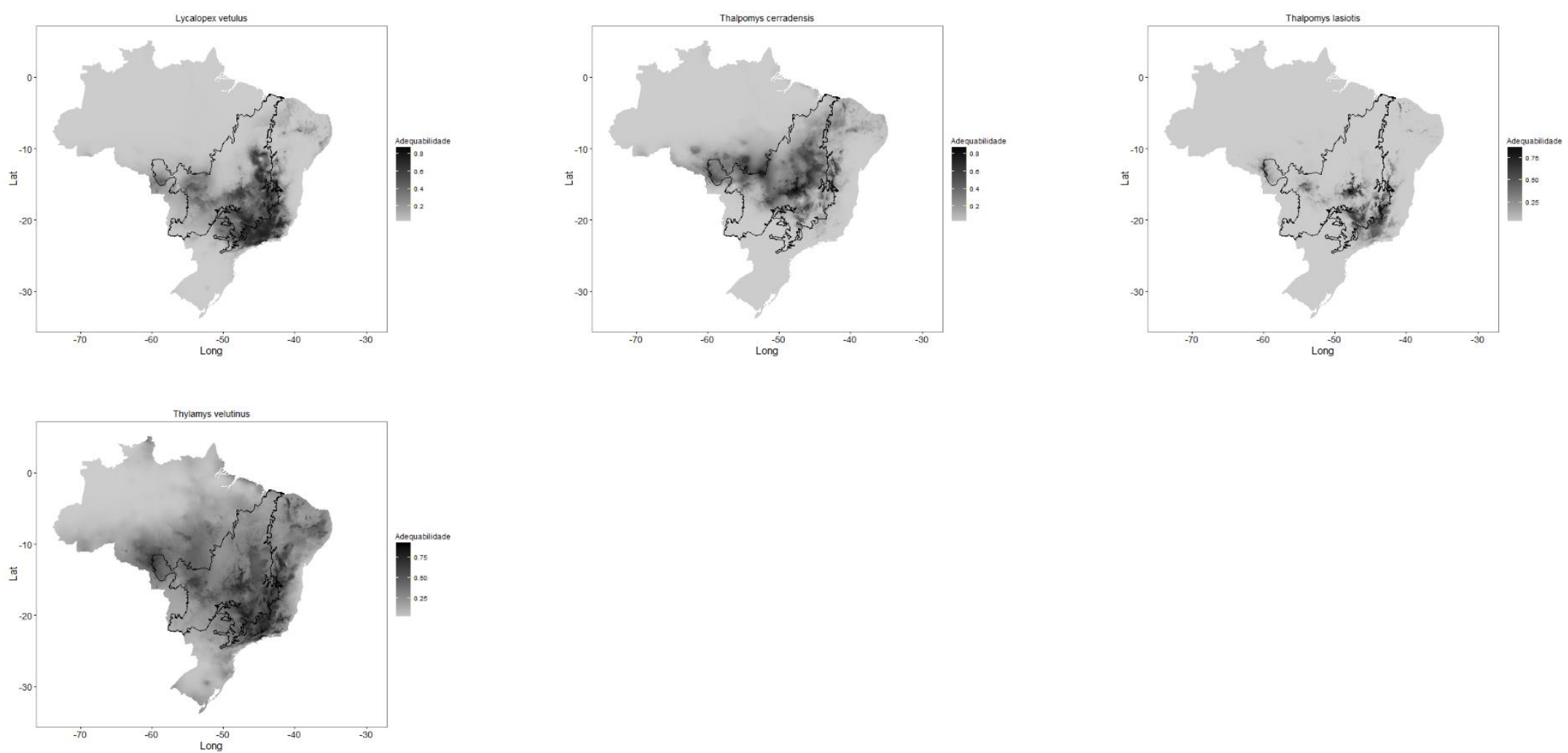

Figura S3: SDMs das espécies-alvo. 


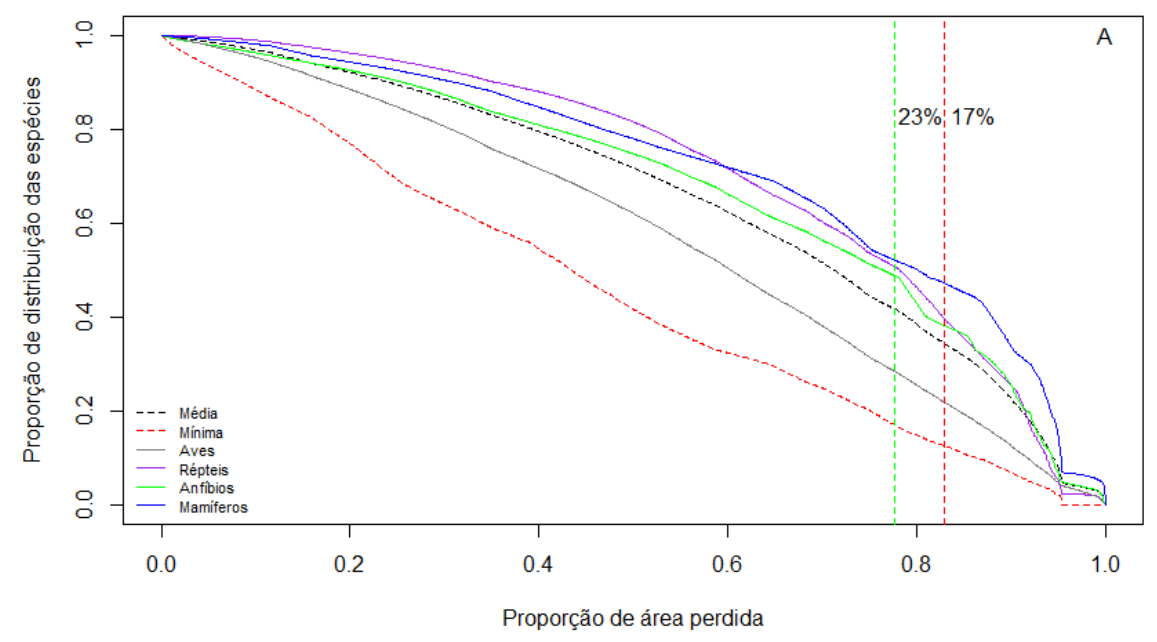

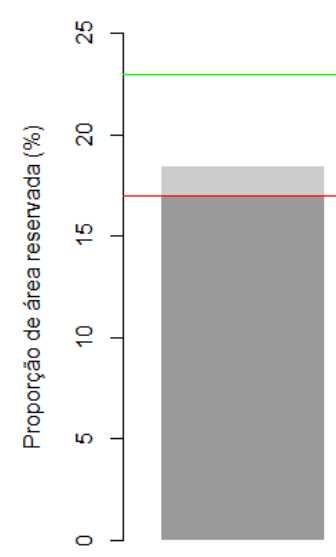

sp1

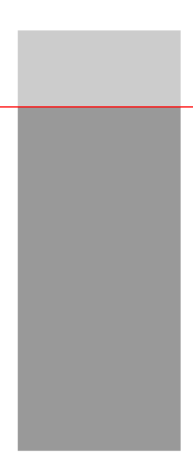

sp2

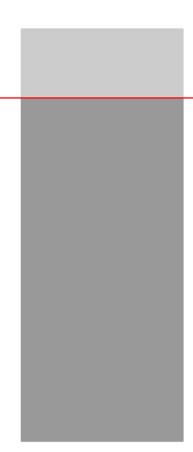

sp3

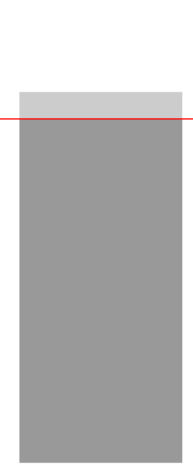

$\mathrm{sp} 4$

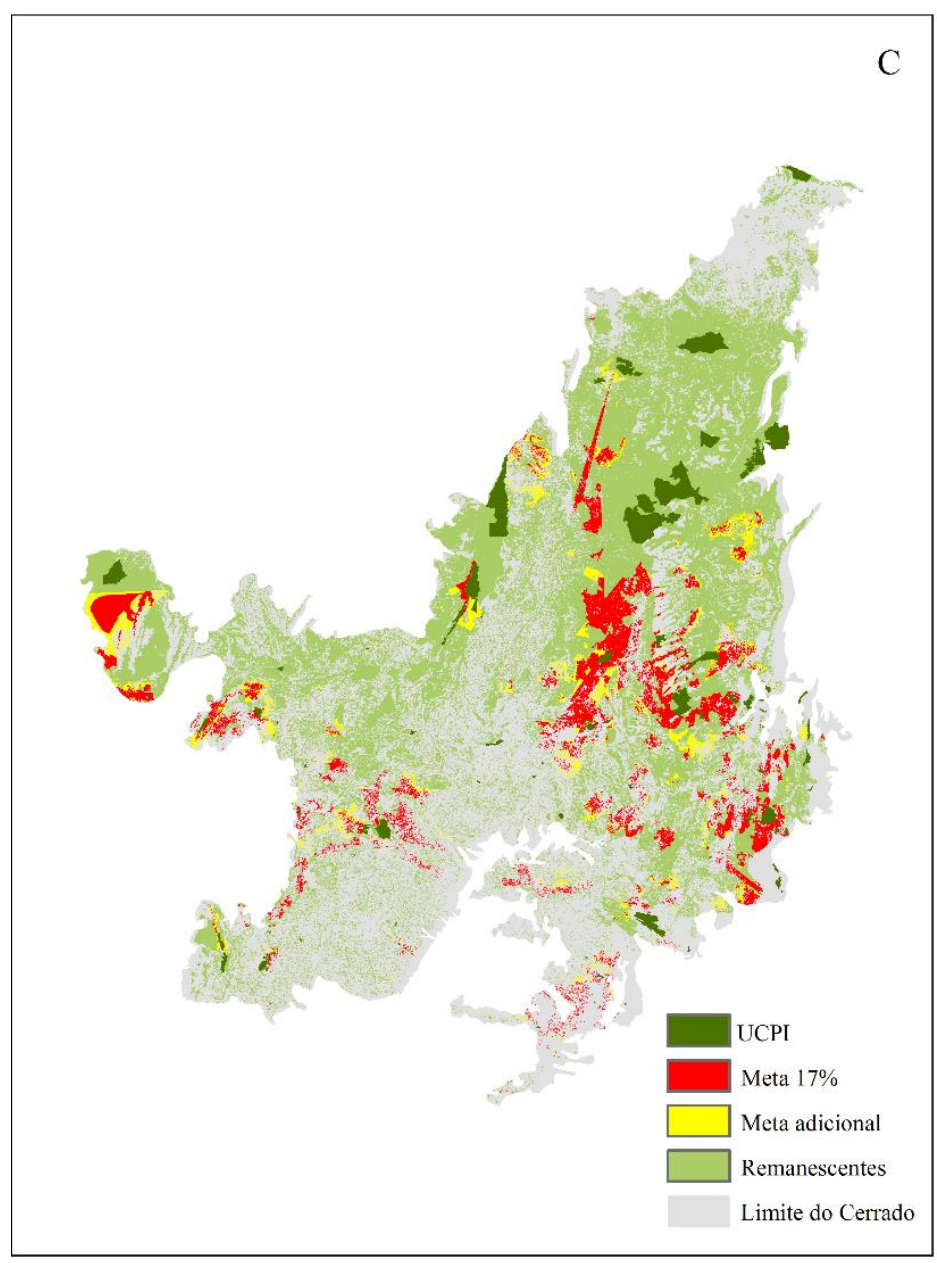

Figura S4: Cenário GOV 2010-2020. A - curvas mostrando a proporção da área de distribuição das espécies em função da proporção de áreas perdidas, a linha tracejada vermelha indica as áreas selecionadas de acordo com as metas de Aichi (CDB, 2013) e a linha tracejada verde, indica a área complementar, área mínima do SDMs das espécies-alvo para reserva de 17\%. B - as barras em cinza claro indicam a meta adicional para cada uma das espécies lacunas, para que elas tenham 17\% de seus SDMs reservados. C - Áreas priorizadas utilizando as espécies-alvo. 

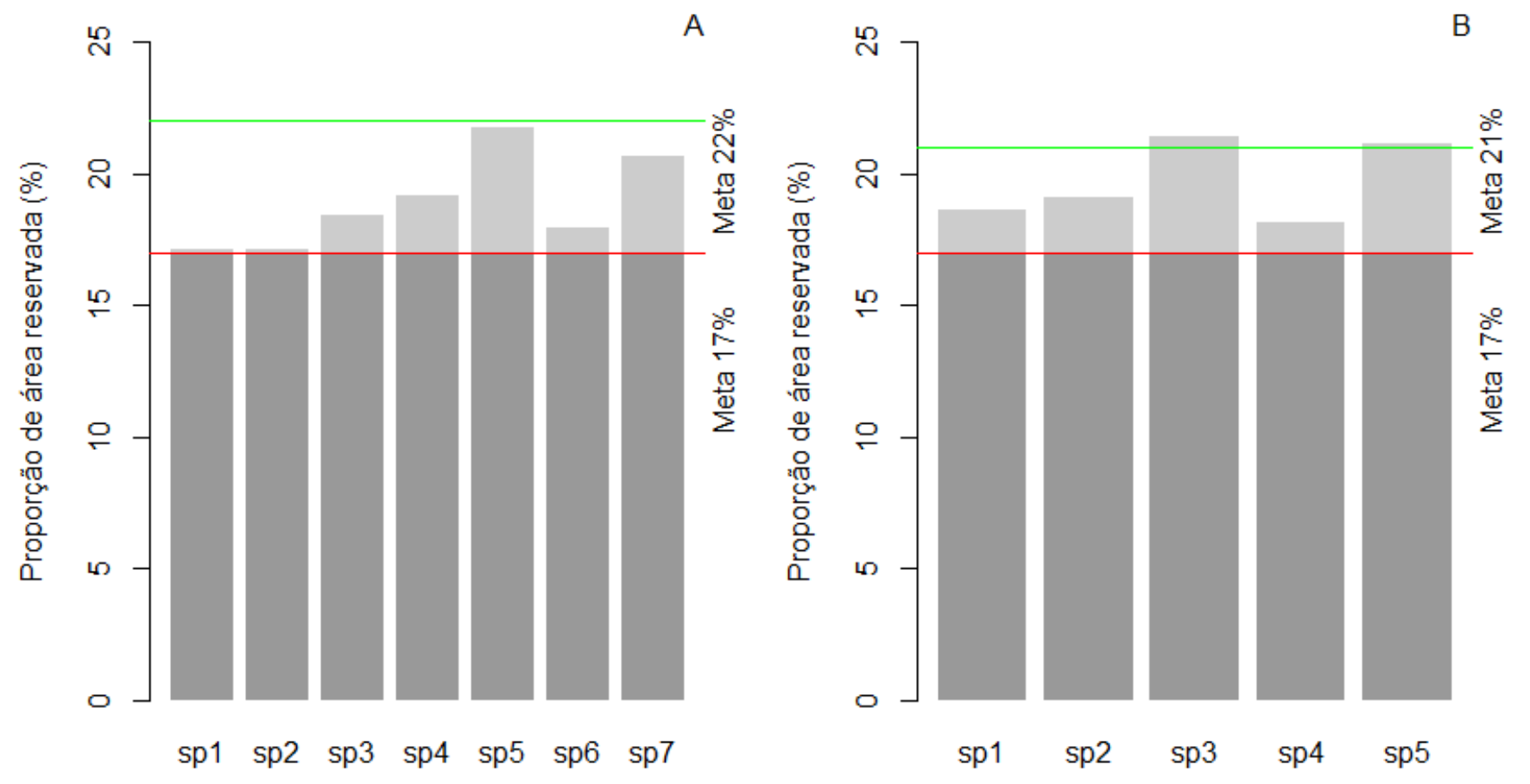

Figura S5: as barras em cinza claro indicam a meta adicional para cada uma das espécies lacunas, para que elas tenham 17\% de seus SDMs reservados. A - Cenário BAU 2010 - 2050 e B - Cenário GOV. 2010 - 2050. 


\section{CONSIDERAÇÕES FINAIS}

As metodologias de seleção sistemática fornecem um arcabouço que permitem aliar o conhecimento científico à prática da conservação. A utilização dessas ferramentas no Brasil foi impulsionada pelas ONGs, sendo posteriormente adotadas como protocolo para seleção de áreas prioritárias pelo governo federal, atendendo a prerrogativa da $\mathrm{CDB}$, para que as futuras áreas reservadas para a conservação fossem representativas da biodiversidade.

Desde a década de 70, o Brasil faz uso do planejamento da conservação, mas os conflitos pelo uso do território tornaram árdua a tarefa de complementação do sistema de UCs. O avanço computacional também contribuiu muito para a mudança dessa cultura do planejamento, primeiro com advento dos sistemas de informação geográficas e, posteriormente, com os algoritmos iterativos de suporte à decisão, baseados no conceito da complementaridade. Além disso, o crescente aumento da disponibilização das bases de dados, também promoveram as mudanças de paradigmas aqui descritas. Mas é importante ressaltar, que nesse processo, os softwares e as informações sistematizadas não substituíram o conhecimento do especialista, que continuou a ter um papel fundamental nas várias etapas da tomada de decisão (Knight et al., 2006; Sarkar et al., 2006).

A ciência busca equacionar o problema da representação da biodiversidade neste tipo de planejamento, mas de um modo geral, ainda há muitos vieses quanto à seleção de alvos, como o uso de espécies e de medidas de riqueza tradicionais. Ainda há uma grande dificuldade de incorporar processos ecológicos como alvos. Também, encontramos poucos estudos que se debrucem sobre o sucesso da implementação dos planos já elaborados.

Nossos estudos buscaram entender sobre como a ciência do planejamento sistemático da conservação evoluiu em nosso território, desde a reflexão científica até sua aplicação prática. Depois, utilizando o Cerrado como modelo, elaboramos cenários a partir dos compromissos do Brasil pós-CDB. Utilizando modelos de LUCC, espacializamos estes cenários para antecipar o desmatamento do nosso território. Mantidas as atuais tendências de desmatamento, nossos cenários mostram que em 2050, cerca de $72 \%$ das áreas nativas seriam perdidas e o cenários que preveem uma queda de 50\% mostram uma redução de 11,5\% dessa perda. Os cenários também revelam que somente a imobilização das grandes áreas remanescentes aos norte dissociadas de ações de comando e controle, que podem manter os pequenos fragmentos, principalmente nas porções centro-sul do bioma, seriam insuficientes para manter a representatividade dos alvos selecionados neste estudo. $\mathrm{E}$ as metas lineares de $17 \%$ seriam insuficientes para a representação de espécies endêmicas e ameaçadas, sobretudo aqueles com pequenas áreas de distribuição. 
Os modelos futuros da dinâmica de uso e cobertura da terra (LUCC) e as mudanças climáticas ainda são temas de poucas pesquisas, sobretudo no Cerrado. A persistência das espécies e dos ecossistemas podem ser garantidas de muitas formas, mas a perda de hábitats é o principal desafio para o desenho de reservas. Os modelos preditivos, nos permitem extrapolações teóricas, seja para complementar registros; para projetar tendências futuras, a partir de cenários planejados ou; para reservar áreas importantes para determinados alvos da conservação. O desenvolvimento de cenários, pode auxiliar na tomada de decisão, mas para um maior sucesso na obtenção de resultados que se aproximem da realidade, são necessários dados e pesquisas que garantam robustez a esses prognósticos.

Avaliar esses esforços pode garantir que o país trilhe os rumos propostos pela Convenção, que ainda é uma tarefa difícil, já que os programas e políticas de conservação não possuem continuidade, são pouco estratégicos e, muitas vezes, são implementados em oportunidades isoladas. O que reflete a produção de indicadores para o monitoramento, em sua maioria, heterogêneos, desagregados e sem padronização (Han et al., 2014). O objetivo dos indicadores vai além do subsídio aos cientistas e tomadores de decisão, uma vez que são importantes ferramentas de comunicação social, pois ilustram a complexidade das informações ambientais para a sociedade em geral (Xu et al., 2009). O Brasil tem a oportunidade como signatário da CDB, de planejar, executar e monitorar quão efetivas são suas ações para a conservação, reavaliando suas metas nacionais e como contribuímos para a manutenção da biodiversidade global.

\section{REFERÊNCIAS BIBLIOGRAFICAS}

Han, X., Smyth, R. L., Young, B. E., Brooks, T. M., De Lozada, A. S., Bubb, P., .. Turner, W. R. (2014). A biodiversity indicators dashboard: Addressing challenges to monitoring progress towards the Aichi biodiversity targets using disaggregated global data. PLoS ONE, 9(11). http://doi.org/10.1371/journal.pone.0112046

Knight, A. T., Driver, A., Cowling, R. M., Maze, K., Desmet, P. G., Lombard, A. T., ... Von Hase, A. (2006). Designing Systematic Conservation Assessments that Promote Effective Implementation: Best Practice from South Africa. Conservation Biology, 20(3), 739-750. http://doi.org/10.1111/j.15231739.2006.00452.x

Sarkar, S., Pressey, R.L., Faith, D.P., Margules, C.R., Fuller, T., Stoms, D.M., Moffett, A., Wilson, K. a., Williams, K.J., Williams, P.H., Andelman, S. (2006). Biodiversity Conservation Planning Tools: Present Status and Challenges for the Future. Annu. Rev. Environ. Resour. 31, 123-159. doi:10.1146/annurev.energy.31.042606.085844

Xu, H., Tang, X., Liu, J., Ding, H., Wu, J., Zhang, M., Yang, Q., Cai, L., Zhao, H., Liu, Y. (2009). China's Progress toward the Significant Reduction of the Rate of Biodiversity Loss. Bioscience 59, 843-852. doi:10.1525/bio.2009.59.10.6 